Portland State University

PDXScholar

Winter 3-26-2018

\title{
Assessment of Technology Adoption Potential of Medical Devices: Case of Wearable Sensor Products for Pervasive Care in Neurosurgery and Orthopedics
}

Liliya Stepanivna Hogaboam

Portland State University

Follow this and additional works at: https://pdxscholar.library.pdx.edu/open_access_etds

Part of the Medicine and Health Sciences Commons, and the Technology and Innovation Commons Let us know how access to this document benefits you.

\section{Recommended Citation}

Hogaboam, Liliya Stepanivna, "Assessment of Technology Adoption Potential of Medical Devices: Case of Wearable Sensor Products for Pervasive Care in Neurosurgery and Orthopedics" (2018). Dissertations and Theses. Paper 4205.

https://doi.org/10.15760/etd.6093

This Dissertation is brought to you for free and open access. It has been accepted for inclusion in Dissertations and Theses by an authorized administrator of PDXScholar. Please contact us if we can make this document more accessible: pdxscholar@pdx.edu. 
Assessment of Technology Adoption Potential of Medical Devices: Case of Wearable Sensor Products for Pervasive Care in Neurosurgery and Orthopedics

by

Liliya Stepanivna Hogaboam

A dissertation submitted in partial fulfillment of the requirements for the degree of

\author{
Doctor of Philosophy \\ in \\ Technology Management
}

Dissertation Committee:

Tugrul U. Daim, Chair

Timothy Anderson

Robert Fountain

David Sibell

Portland State University

2018 
(C) 2018 Liliya Stepanivna Hogaboam 


\begin{abstract}
Information and communication technologies hope to revolutionize the healthcare industry with innovative and affordable solutions with a focus on pervasive care. Wearable sensors products can provide monitoring in a natural environment with a constant stream of information, enriching healthcare practices and enabling better pervasive care.
\end{abstract}

Wearable sensor technologies could monitor patients' mobility, gait, tremor, daily activity and other health indicators in real time that could allow for simple, non-invasive, tracking of spine care that may lead to increased patient engagement, integration, feedback, post-surgery analysis, monitoring of patient's condition, patient's data extraction and analysis and possibly aiding in better diagnosis, intervention, adherence to treatment for the betterment of quality of care.

This research focuses on the assessment of technology adoption potential of medical devices particular to tracking the mobility of patients of neurosurgery and orthopedics.

Wearable medical devices that track the mobility of patients after spinal procedures could help surgeons in providing post-operative care, analysis of treatment outcomes and patient mobility. The assessment of those devices by physicians is a complex process associated with various perspectives and criteria. 
Therefore, the objective of this research is to assess the potential for technology adoption of those wearable medical devices through development of a hierarchical decision-making model (HDM) that incorporates the relevant perspectives and criteria encompassing the needs of hospital neurological surgery and orthopedics departments.

The proposed research builds on an existing body of knowledge researched through literature review and background of the field and expands the health technology assessment field by implementation of a holistic, comprehensive and multi-perspective approach to technology assessment in wearable sensor products adoption for pervasive care in neurosurgery and orthopedics.

The Hierarchical Decision Model (HDM) approach is used to break the problem down into hierarchical levels and then calculate the alternatives using pairwise comparison scales and a judgment quantification technique. Inconsistencies, disagreement, sensitivity and scenario analysis are performed as well. HDM research software is created with Ruby and R to facilitate the computation of some of these important model parameters to higher precision than is available in current statistical analysis software packages or extensions targeted for decision making. Patient perspective dominates as the main perspective for the technology adoption potential of wearable devices for pervasive care in neurosurgery and orthopedics, followed by technical and financial perspectives. Valedo, a wearable device aimed to relieve back pain 
through exercises, motivation and mobility tracking, received the highest ranking for adoption potential, while other devices also received high relative scores. The framework could serve as a supplementary technology assessment tool and could be tested in other settings: private, small clinic etc. with the experts and special needs of physicians in particular healthcare departments. 


\section{DEDICATION}

To my wonderful parents, Stepan and Larysa Yaremchuk, who always supported me and encouraged me to achieve my dreams. To my grandparents, especially my grandma Claudia, who nurtured the qualities in me that led me to this accomplishment.

To my loving and caring family, my daughter Octavia and my husband Justin, who patiently tolerated and supported me through all the ups and downs, with unconditional love and inspiration. I truly cherish friends and family, who encouraged me and inspired me. 


\section{ACKNOWLEDGEMENTS}

I would like to thank and acknowledge many individuals, who provided support and guidance in my research over the years.

I would like to express my gratitude to my advisor, Dr. Tugrul U. Daim for his guidance, mentorship, patience, understanding and encouragement in the challenges of this research process. He is a great inspirational leader and he deeply cares about his students. Thank you for listening, caring, responding and advising during all those years. I really appreciate it!

I received great support from my committee. I would like to thank Dr. Anderson for his support and encouragement, and great conversations on topics of programming, software packages and R. I am grateful to Dr. Fountain for his kind support, understanding and reassurance in this uneasy research process. Dr. Sibell was always happy to meet with me, and provided great help and support in the field of healthcare informatics. I really appreciate his questions and feedback, which helped me during the research process. Thank you!

I would like to thank our department's founding father, Dr. Kocaoglu, for sharing his wisdom, experience and methodology. I appreciate the encouragement, support and feedback in the model development stages of my research. 
It was amazing to receive the support of OHSU neurosurgery and orthopedics departments. The surgeons and staff at OHSU neurosurgery and orthopedics departments have shared their experience, provided their expert opinion and I am grateful for their help, meetings, encouragement and feedback throughout this study. Dr. Ragel introduced me to the problems in spine health and the needs of surgeons in neurosurgery and orthopedics, opened a view into the life of surgeons and supported me through my research. I appreciate all your help. Dr. Raslan for helped me in the model development stage and discussed healthcare technology assessment field. Thank you! I am grateful to Dr. Marshall for helping me understand the problems in spine health, introducing me to Spine Center and outcomes database at OHSU and providing feedback during the model development stage of my research.

Engineering and Technology Management department at PSU has really great people, leaders in their field and I am proud that I had the opportunity to research in this environment. I received kind support and encouragement over the years and I would like to thank all faculty, staff and friends at the ETM department.

It would be much harder to accomplish this milestone without the support of my close and extended family and friends, who believed in me, inspired me and encouraged me every step of the way. Thank you! My grandpa Anatoliy and my grandma Claudia always encouraged me and knew I could achieve this, even though they didn't get to see it. I really appreciate your love and inspiration! 
I want to thank my husband, Justin, for his expertise in computer science, particularly in R and Ruby and for help in troubleshooting $\mathrm{R}$ code with me and the heated discussions on coding with matrices and hierarchies. Thank you for being patient with me, supporting me and loving me during those times.

Thank you, Mom, for your advice, guidance, love, care, inspiration and for making me a strong woman and a fighter! I clearly remember the time when you worked on your dissertation and defense and it was very inspiring.

My darling daughter, Octavia, I want to thank you for your love, support and encouragement. I want you to fight for your dreams, accomplish great things, be happy and have an easier time achieving it.

My appreciation goes out to all the experts, who participated in this study. Your time devoted to effort in filling out the questionnaire, providing conversations and feedback is greatly appreciated. Thank you! 


\section{TABLE OF CONTENTS}

ABSTRACT

i

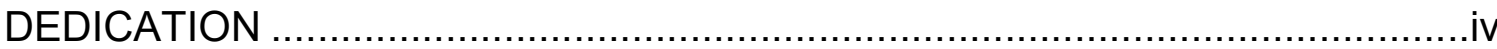

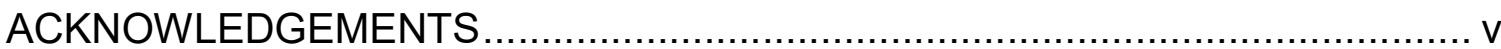

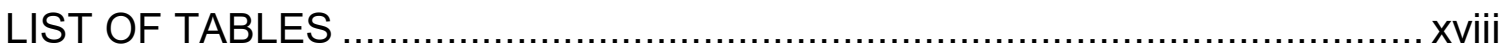

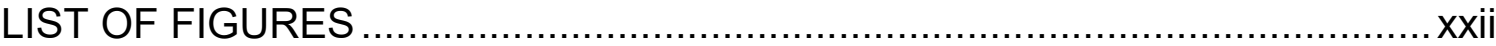

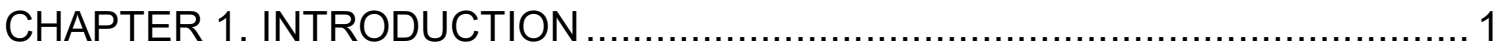

CHAPTER 2. LITERATURE REVIEW ......................................................... 4

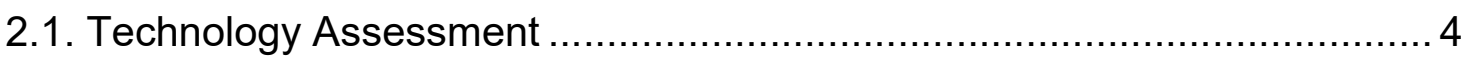

2.1.1. System dynamics and structural modeling ................................... 5

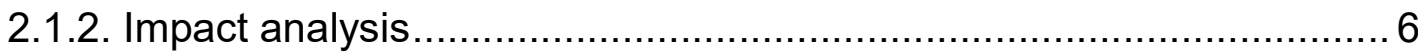

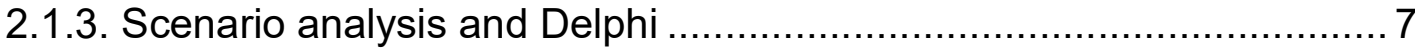

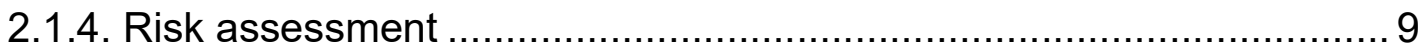

2.1.5. Multi-Criteria Decision Analysis (MCDA) …................................. 9

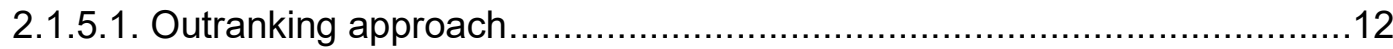

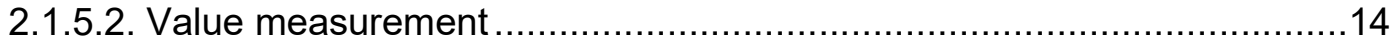

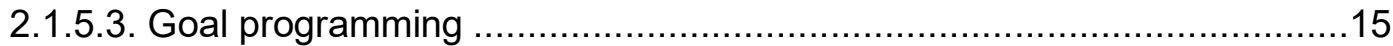

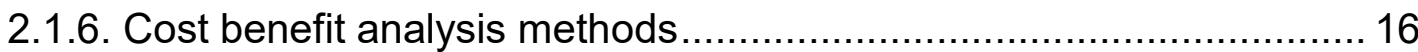

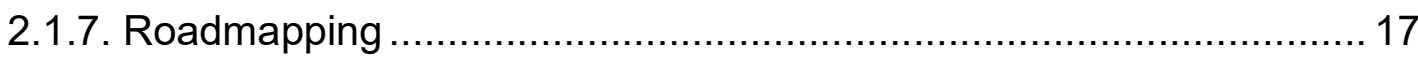

2.1.8. Mathematical and synthesis methods......................................... 18 


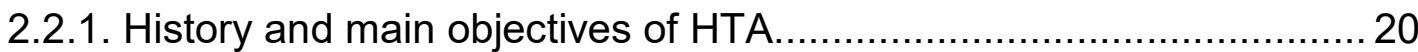

2.2.2. Problems in technology diffusion in healthcare ............................. 21

2.2.3. Main steps in HTA and impacts on technology implementation ........ 24

2.2.4. Use of scenarios in healthcare technology assessment .................. 26

2.2.5. Healthcare assessments based on outcome studies...................... 27

2.2.6. Decision-making methodologies in healthcare ............................. 27

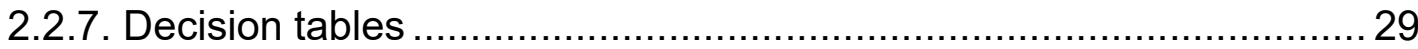

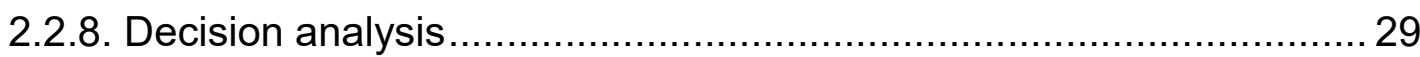

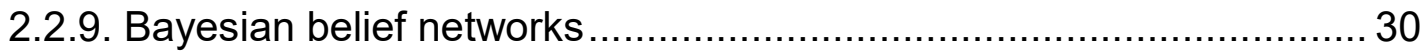

2.2.10. Other technology assessment techniques ................................... 30

2.2.11. Database prediction: data mining and machine learning ................ 31

2.2.12. Applications of hierarchical decision modeling in healthcare........... 33

2.2.13. HTA in the United States compared to other countries................... 34

2.3. Health Information Technology Assessment and Adoption ..................... 38

2.3.1. Theoretical approaches the conceptualize interaction of technology,

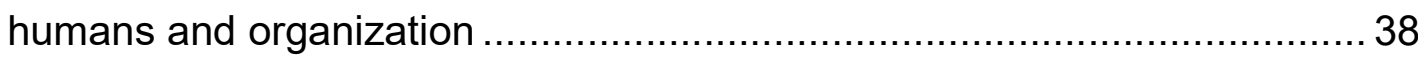

2.3.2. Statistics of HIT adoption and diffusion ........................................ 41

2.3.3. Barriers to adoption in health information technology studies .......... 43 


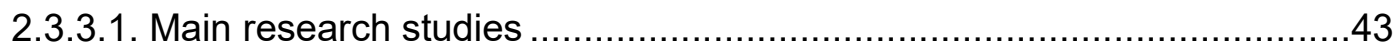

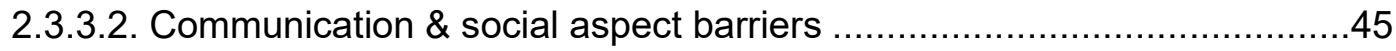

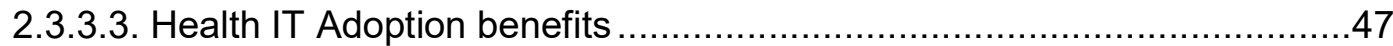

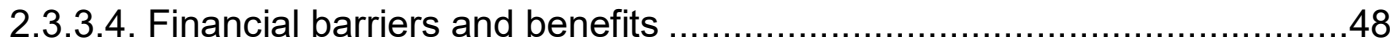

2.3.4. Technology Acceptance Model: applications, extensions and SEM in

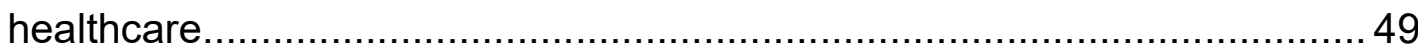

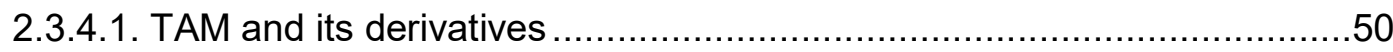

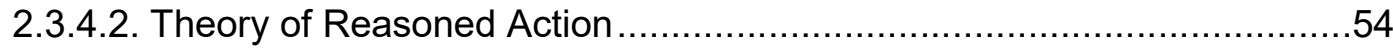

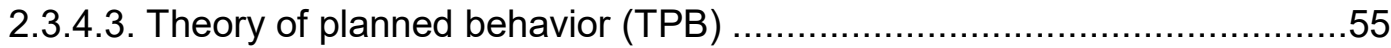

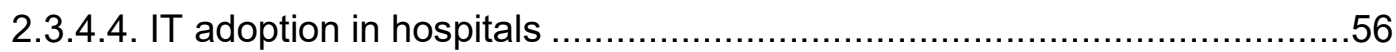

2.3.5. Other health technology assessment methods............................... 63

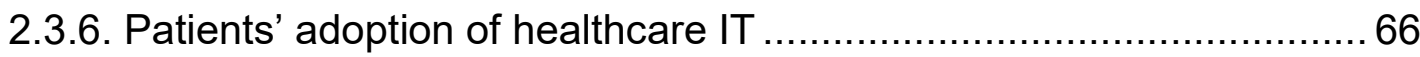

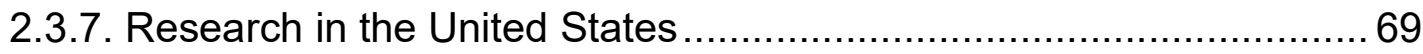

2.3.8. HIT Adoption in other countries .............................................. 71

2.3.9. Quality Indicators in Healthcare Adoption and their Impacts ............ 72

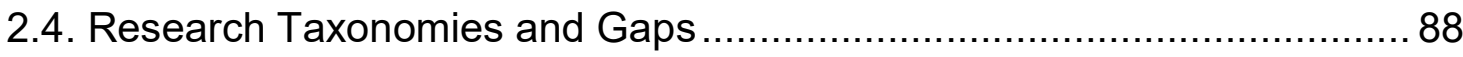

2.4.1. Taxonomy of Technology Assessment methods ............................ 88

2.4.2. Taxonomy of Health Technology Assessment methods................... 89

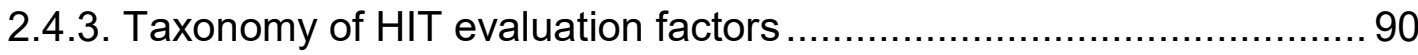

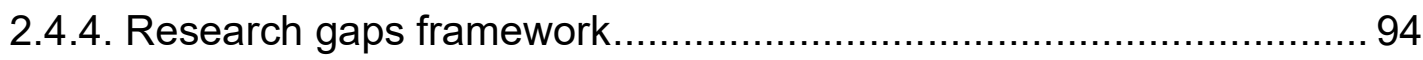

CHAPTER 3. RESEARCH METHODOLOGY ……...................................... 97

3.1. Validation of Hierarchical Decision Modeling through Applications .......... 97 
3.2. Hierarchical Decision Model (HDM) ............................................... 102

3.3. Generalized Hierarchical Decision Model............................................. 103

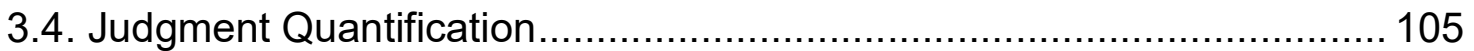

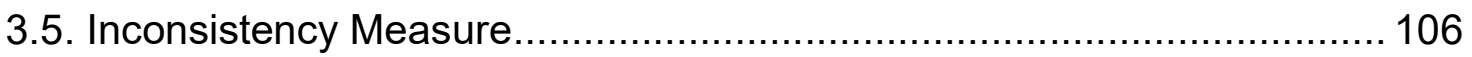

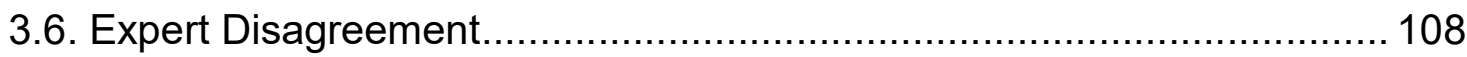

3.7. Sensitivity Analysis ...................................................................... 110

3.8. Expert Panel Design................................................................... 113

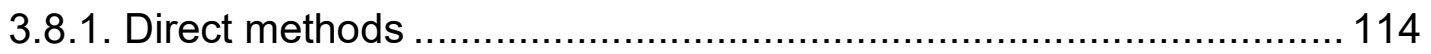

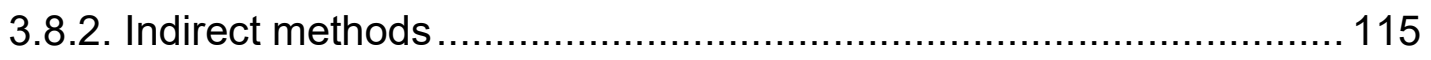

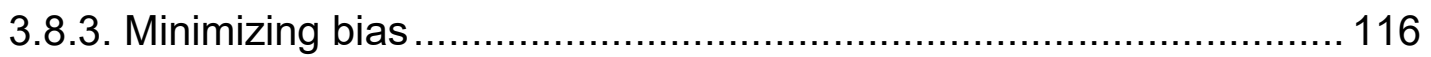

3.8.4. Focus and expertise of expert panels........................................ 117

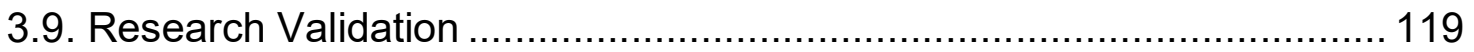

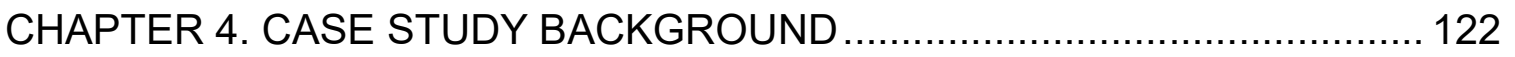

4.1. Mobile Healthcare Systems ........................................................ 122

4.2. Importance of Patient-Oriented Intelligible Systems........................... 123

4.3. Preventative and Integrated Care Systems ...................................... 125

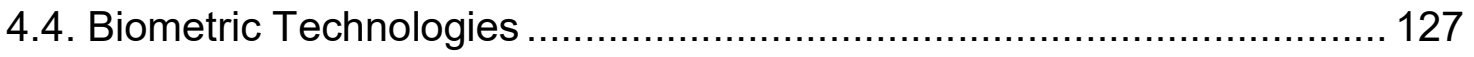

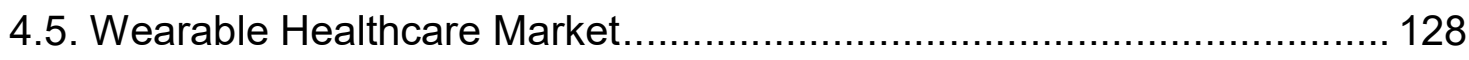

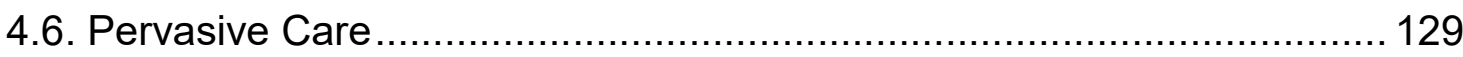


4.7. Wearable Medical Systems

4.8. Medical Sensors and Targeted Applications for Patient Monitoring ....... 133

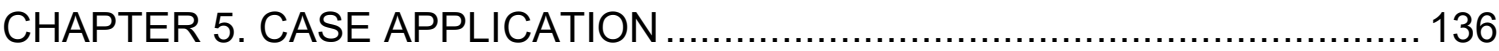

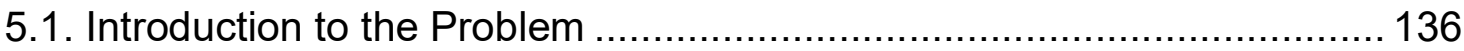

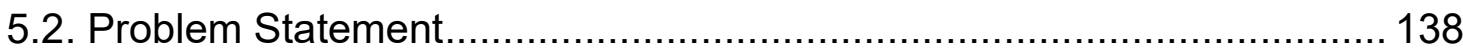

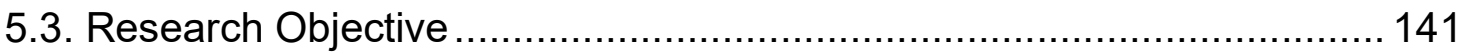

CHAPTER 6. CASE STUDY MODEL DEVELOPMENT .............................. 144

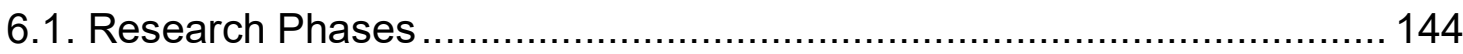

6.1.1. Phase 1: Literature analysis and HDM model development........... 144

6.1.2. Phase 2: Instrument development ............................................ 144

6.1.3. Phase 3: Data collection and judgment quantification ................... 145

6.1.4. Phase 4: Data analysis, conclusions, outlining recommendations .. 145

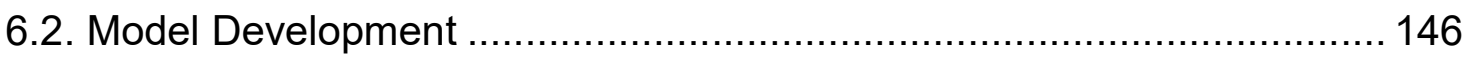

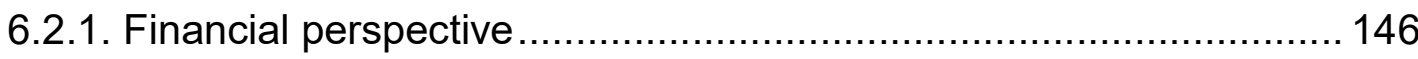

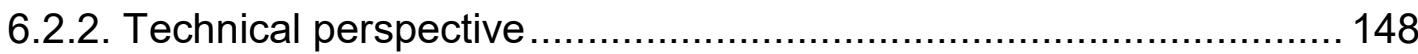

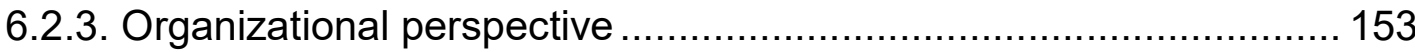

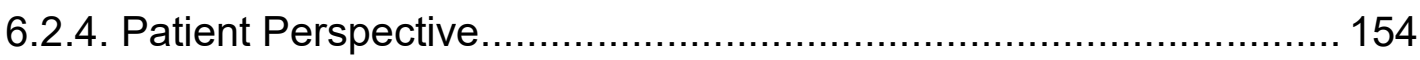

6.2.5. Interpersonal perspective .................................................... 156

6.2.3. Taxonomy of the main model perspectives ................................ 157 
6.2.4. Taxonomies of model criteria 160

6.2.5. Research focus 166

6.2.6. Alternatives - wearable sensor devices 167

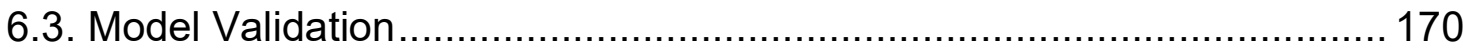

6.4. Finalized Assessment Model........................................................ 178

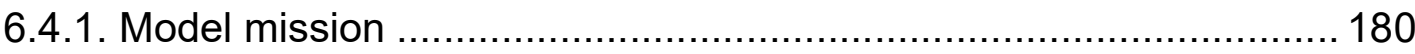

6.4.2. Model perspectives and criteria ............................................ 180

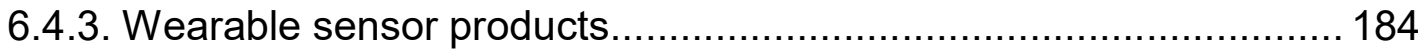

6.5. Data Collection and Expert Panels Design........................................ 188

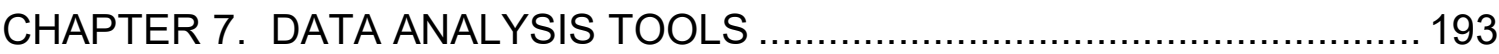

7.1. Hierarchical Decision Modeling Research Software Package ............... 193

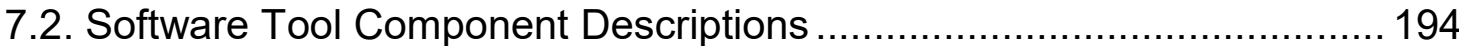

7.2.1. Qualtrics questionnaire instrument .......................................... 194

7.2.2. Qualtrics2ahp data processing utility ....................................... 194

7.2.3. R CRAN AHP package customized script ................................... 196

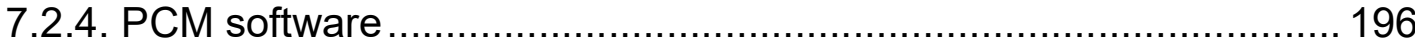

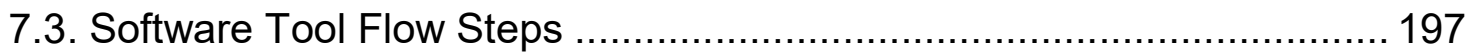

7.3.1. Saving survey response panel data from Qualtrics to XML ............ 197

7.3.2. Using Ruby qualtrics2ahp.rb to convert XML to HDM format .......... 197 
7.3.3. Generating HDM in R using AHP module. 198

7.3.4. Analyzing inconsistencies. 199

7.3.5. Analyzing disagreement and cluster analysis in $\mathrm{R}$ 199

CHAPTER 8. RESULTS AND DATA ANALYSIS ….................................. 200

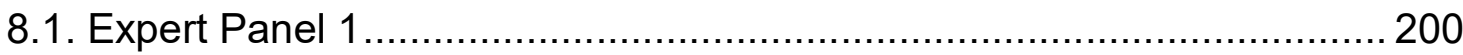

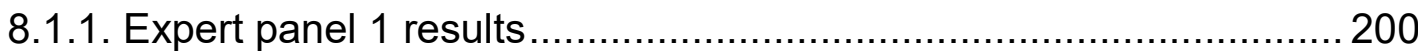

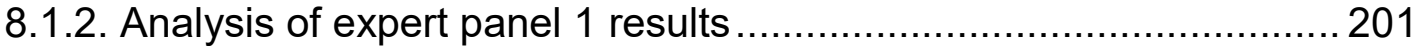

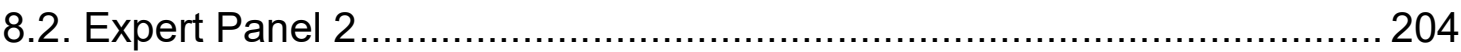

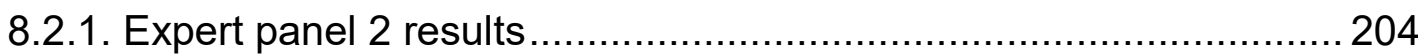

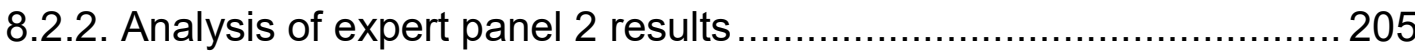

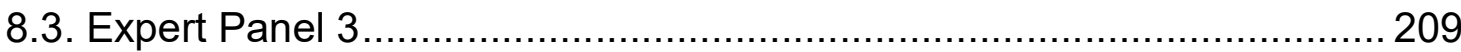

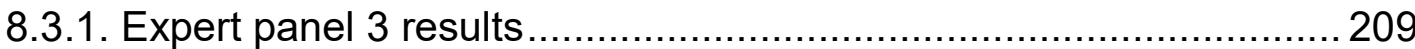

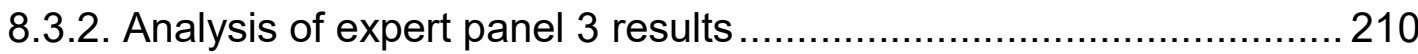

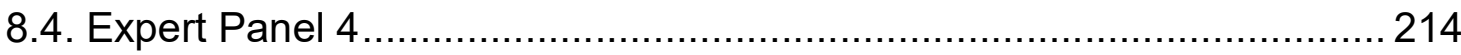

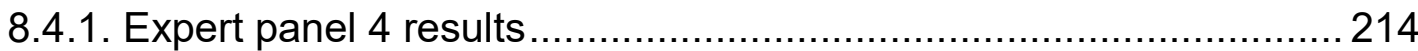

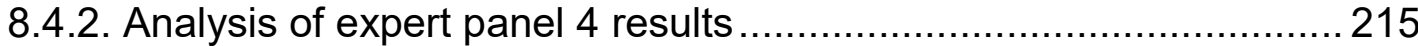

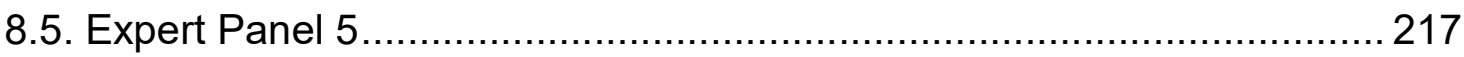

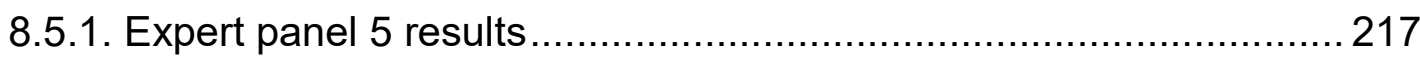

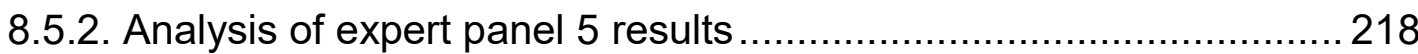


8.6. Expert Panel 6 .

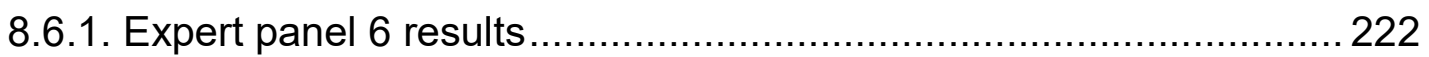

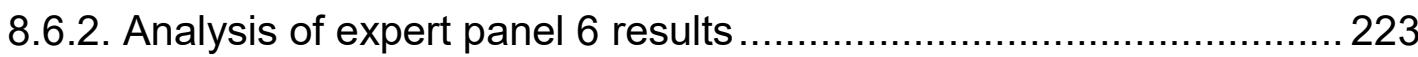

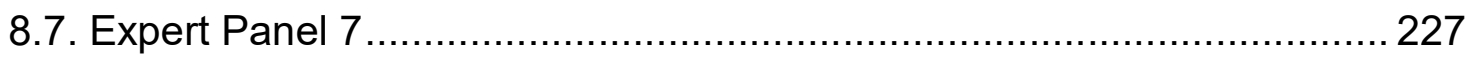

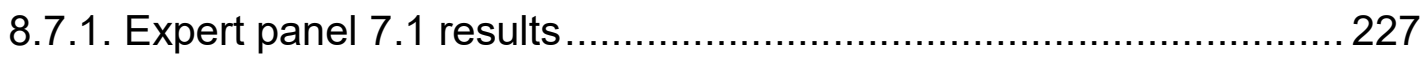

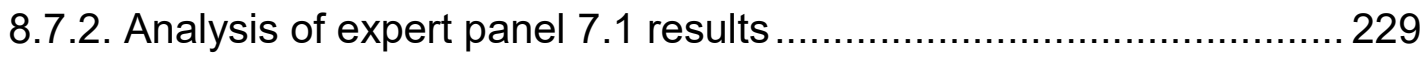

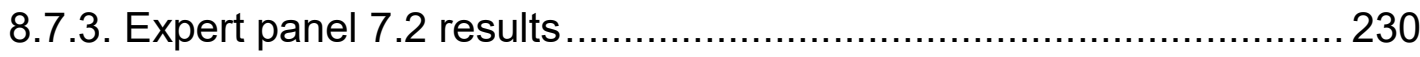

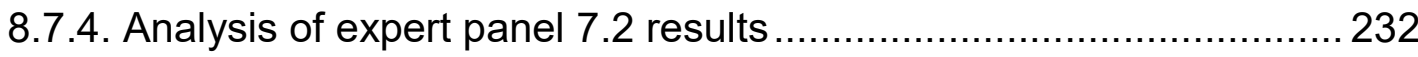

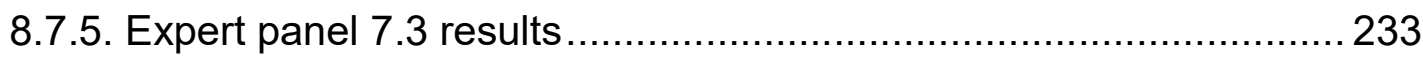

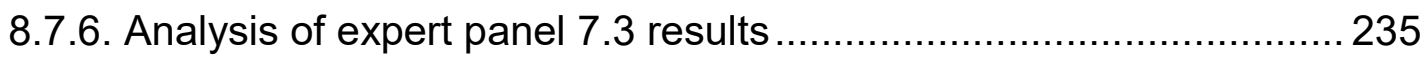

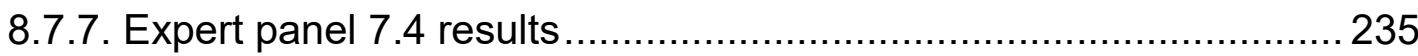

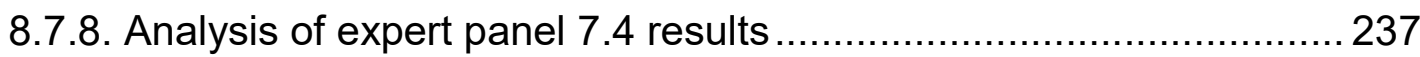

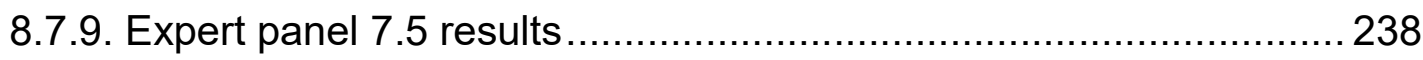

8.7.10. Analysis of expert panel 7.5 results ........................................ 239

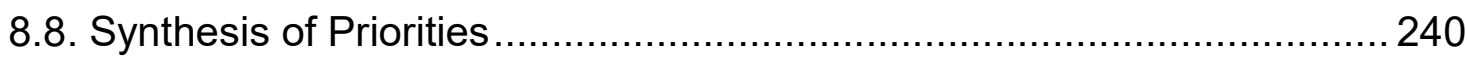

8.8.1. Relative importance of criteria with respect to the mission .............. 241

8.8.2. Relative importance of alternatives with respect to perspectives. ... 242

8.8.3. Overall importance of alternatives with respect to the mission........ 247 
8.9. Analysis of Ranking of Model Alternatives with Respect to Expert Panels Disagreements 249

8.9.1. Analysis of results with respect to expert disagreements in panel 1249

8.9.2. Analysis of results with respect to expert disagreements in panel 2250

8.9.3. Analysis of results with respect to expert disagreements in panel 3251

8.9.4. Analysis of results with respect to expert disagreements in panel 4252

8.9.5. Analysis of results with respect to expert disagreements in panel 5253

8.9.6. Analysis of results with respect to expert disagreements in panel 6255

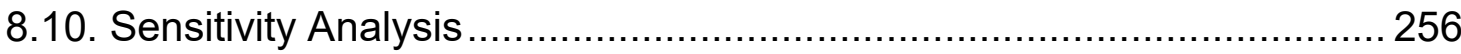

8.10.1. HDM SA at the perspective level to preserve the ranking of the best

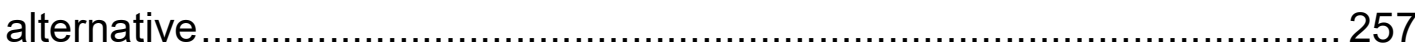

8.10.2. HDM SA at the perspective level to preserve the ranking of all

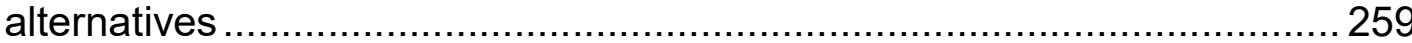

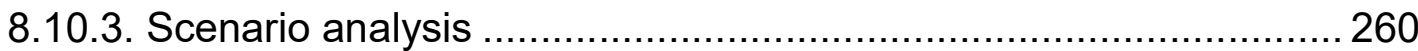

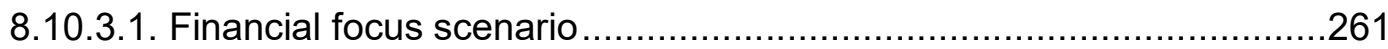

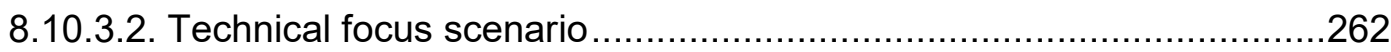

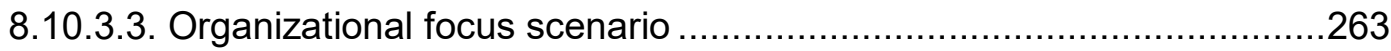

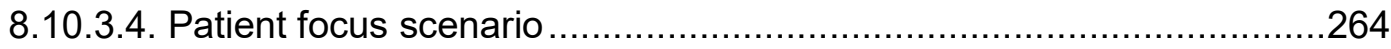

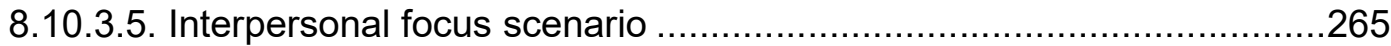

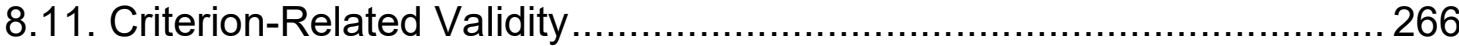

CHAPTER 9. DISCUSSIONS AND RECOMMENDATIONS ......................... 268

9.1. Model Perspectives and Criteria......................................................... 268 
CHAPTER 10. CONCLUSIONS, LIMITATIONS AND FUTURE RESEARCH. 273

10.1. Assumptions and Limitations of the Proposed Research 273

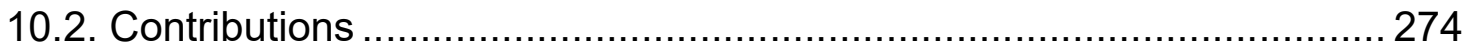

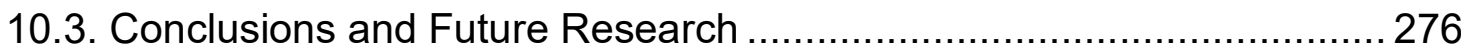

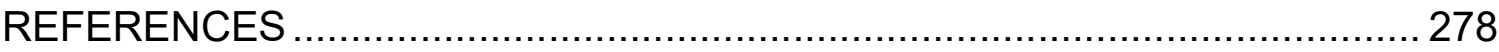

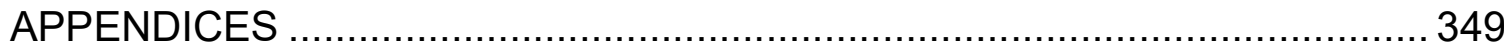

Appendix A - Research Invitation Letter ................................................ 350

Appendix B - Validation Questionnaire .................................................. 352

Appendix C - Quantification Instrument -Qualtrics................................... 361

Appendix D - XML Response File Sample .................................................366

Appendix E - Criteria Mapping xml file for Ruby and R ............................. 367

Appendix F - Sample of Questions Mapping xml file ................................. 368

Appendix G - Sample Hierarchical Decision Model code in R......................369

Appendix H - HDM Research Software module ........................................ 373 


\section{LIST OF TABLES}

Table 1 Technology assessment approaches and methods in public vs private domain (Tran and Daim 2008)........................................................... 5

Table 2 Primary research methodologies in the areas of Clinical Decision Support and Quality/Performance Improvement.......................................... 28

Table 3 Machine learning techniques in Healthcare Analytics.......................... 32

Table 4 Theoretical approaches of interaction between technology humans and

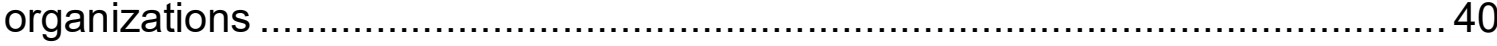

Table 5 Taxonomy of "primary" barriers (adopted from Boonstra and Broekhuis 2010).

Table 6 Problems/barriers in HIT adoption. 48

Table 7 Evaluation criteria for development and integration of the health care professional multimedia workstation based on Degoulet et al. (1995)................ 64

Table 8 Potential benefits and their related features …............................... 76

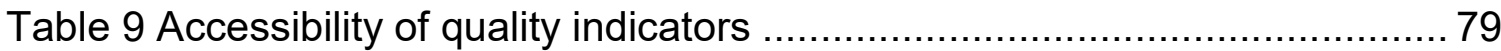

Table 10 Summary points of impact studies (Chaudhry et al. 2006) …............. 83

Table 11 Taxonomy of Technology Assessment methods .............................. 88

Table 12 Taxonomy of Health Technology Assessment Methods ..................... 89

Table 13 Taxonomy of HIT evaluation factors - Financial ...............................90

Table 14 Taxonomy of HIT evaluation factors - Technical ............................. 90

Table 15 Taxonomy of HIT evaluation factors - Social/Organizational - Part 1.91

Table 16 Taxonomy of HIT evaluation factors - Social/Organizational - Part 2.92

Table 17 Taxonomy of HIT evaluation factors - Personal ................................ 93

Table 18 Taxonomy of HIT evaluation factors - Interpersonal .........................93

Table 19 Bibliographical review of types of studies performed in Health

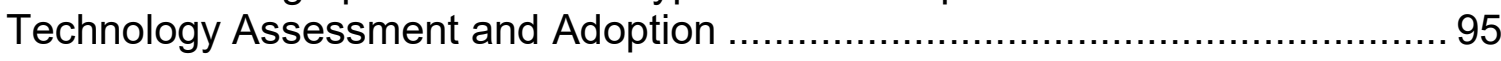


Table 20 Focus and required expertise of expert panels for this research ....... 119

Table 21 Types of validity for the proposed research ................................... 121

Table 22 Pros and Cons of the current OHSU spine patient outcome database

(Integrated Survey System (ISS, Dynamic Clinical Systems, Hanover, NH))... 140

Table 23 Taxonomy of the main perspectives used in the model.................. 158

Table 24 Taxonomy of model criteria of Financial perspective ....................... 160

Table 25 Taxonomy of model criteria of Technical perspective ...................... 161

Table 26 Taxonomy of model criteria of Organizational perspective ............... 163

Table 27 Taxonomy of model criteria of Patient perspective .......................... 164

Table 28 Taxonomy of model criteria of Interpersonal perspective ................. 165

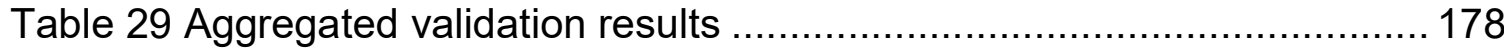

Table 30 Wearable sensor products and their sensor modules...................... 185

Table 31 Expert panels and expertise of the experts................................... 189

Table 32 Quantification panel focus and task ............................................. 191

Table 33 Subpanels of quantification expert panel 7 ................................... 192

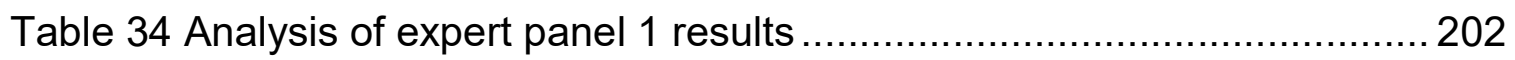

Table 35 Analysis of results in subgroup A of expert panel 1 .........................2 203

Table 36 Analysis of results in subgroup B of expert panel 1 .........................204

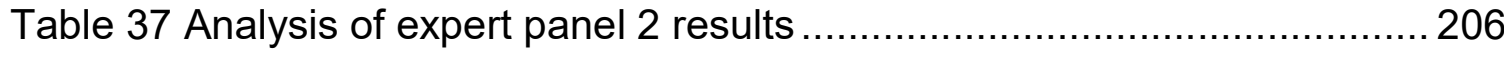

Table 38 Analysis of results in subgroup A of expert panel $2 \ldots \ldots \ldots \ldots \ldots \ldots \ldots \ldots . . . .207$

Table 39 Analysis of results in subgroup B of expert panel 2 ......................... 208

Table 40 Analysis of results in subgroup C of expert panel 2 …..................... 208

Table 41 Analysis of results in subgroup D of expert panel 2 ........................ 209

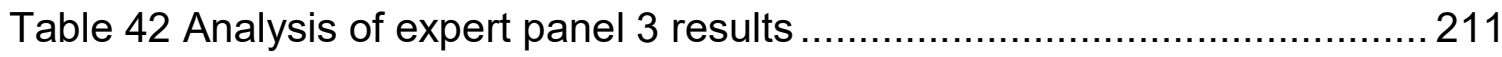


Table 43 Analysis of results in subgroup A of expert panel $3 \ldots \ldots \ldots \ldots \ldots \ldots \ldots \ldots . . .213$

Table 44 Analysis of results in subgroup B of expert panel $3 \ldots \ldots \ldots \ldots \ldots \ldots \ldots \ldots . . .213$

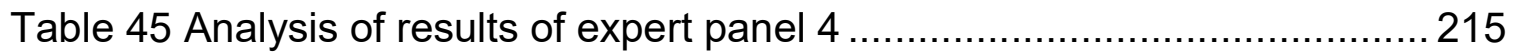

Table 46 Analysis of results in subgroup A of expert panel 4 ........................ 216

Table 47 Analysis of results in subgroup B of expert panel 4 ........................ 217

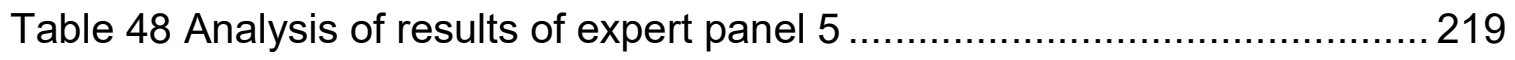

Table 49 Analysis of results in subgroup A of expert panel $5 \ldots \ldots \ldots \ldots \ldots \ldots \ldots \ldots \ldots . . .221$

Table 50 Analysis of results in subgroup B of expert panel $5 \ldots \ldots \ldots \ldots \ldots \ldots \ldots \ldots . . .221$

Table 51 Analysis of results in subgroup $\mathrm{C}$ of expert panel $5 \ldots \ldots \ldots \ldots \ldots \ldots \ldots \ldots . . . . . . . . . .222$

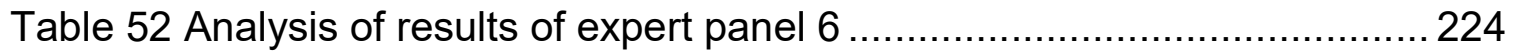

Table 53 Analysis of results in subgroup A of expert panel 6 ........................ 226

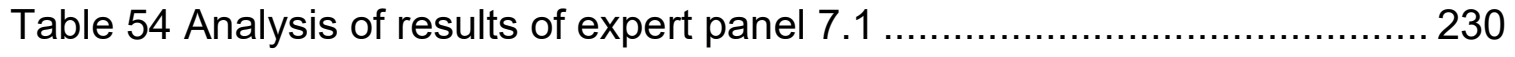

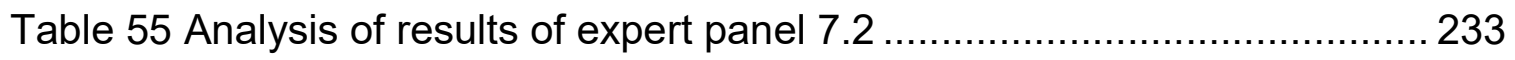

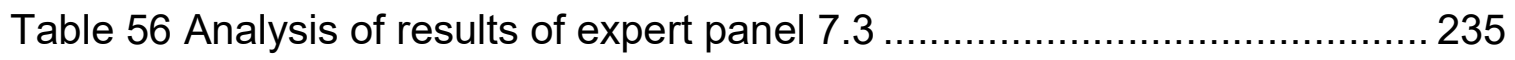

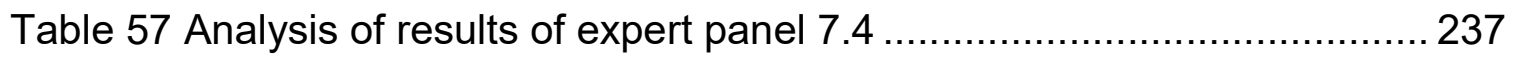

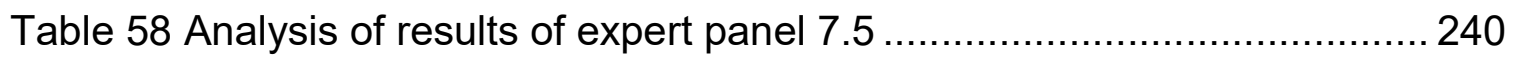

Table 59 Relative importance of perspectives in panel 1 and its subgroups .... 249

Table 60 Global rankings of model alternatives of expert panel 1, subgroups A

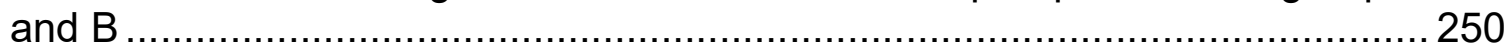

Table 61 Relative importance of criteria in expert panel 2 and its subgroups ... 250 Table 62 Global rankings of model alternatives of panel 2 and subgroups A, B, C

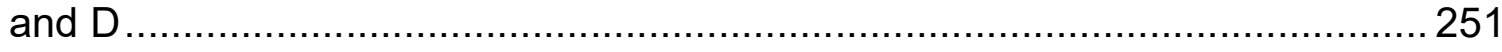

Table 63 Relative importance of criteria in expert panel 3 and its subgroups... 251

Table 64 Global rankings of model alternatives of panel 3 and its subgroups A

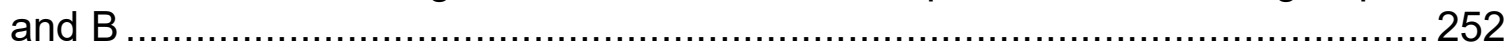


Table 65 Relative importance of criteria in expert panel 4 and its subgroups... 253 Table 66 Global rankings of model alternatives of panel 4 and its subgroups A and $\mathrm{B}$ 253

Table 67 Relative importance of criteria in expert panel 5 and its subgroups... 254 Table 68 Global rankings of model alternatives of panel 5 and its subgroups A, B and $\mathrm{C}$ 254

Table 69 Relative importance of criteria in expert panel 6 and its subgroups... 255 Table 70 Global rankings of model alternatives of panel 6 and subgroups A, B, C

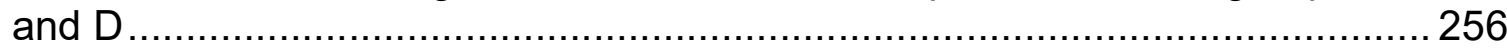

Table 71 Rankings and weights of model alternatives with respect to the mission 257

Table 72 HDM SA at the perspectives level to preserve the ranking of the top alternative 258

Table 73 HDM SA at the perspectives level aimed to preserve the ranking of all alternatives 259

Table 74 Distribution of weights for model perspectives in five scenarios ........ 260

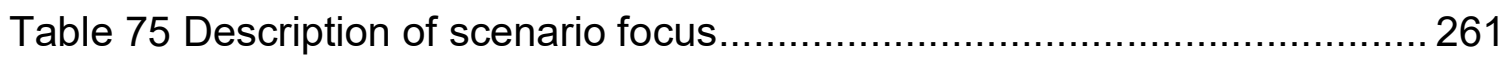




\section{LIST OF FIGURES}

Figure 1 Bodies of knowledge surrounding organizational issues in HIT

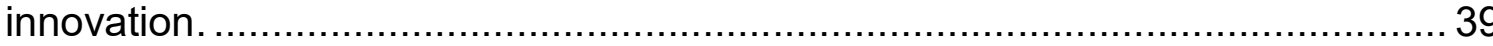

Figure 2 Causal diagram of HIT adoption benefits .......................................47

Figure 3 Model of insulating and risk factors (Ludwick and Doucette 2009)...... 72

Figure 4 Envisioned health information technology benefits............................ 75

Figure 5 Framework of research gaps, goals and questions ......................... 96

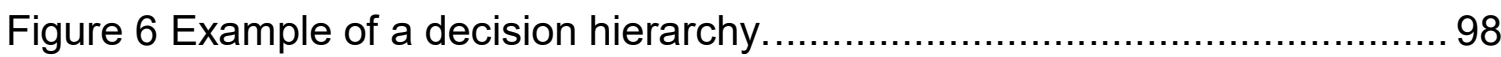

Figure 7 MOGSA research framework (Cleland and Kocaoglu 1981)...............99

Figure 8 Framework for Technological Competitiveness and Innovation (Chan et

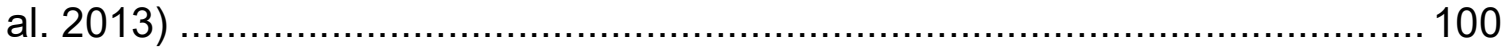

Figure 9 Generalized hierarchical decision model with 4 levels of hierarchy.... 104

Figure 10 Main qualities of the technological system according to the needs physicians

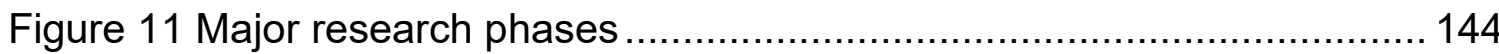

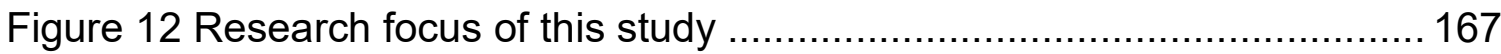

Figure 13 Preliminary model of assessment of Technology Adoption Potential of medical devices................................................................................. 170

Figure 14 Validation of the model Perspectives........................................ 172

Figure 15 Validation of Financial criteria - Panel 2 ....................................... 173

Figure 16 Validation of Technical criteria - Panel 3................................... 173

Figure 17 Validation of Organizational criteria - Panel $4 \ldots \ldots \ldots \ldots \ldots \ldots \ldots \ldots \ldots \ldots \ldots \ldots . . . .174$

Figure 18 Validation of Patient criteria - Panel 5 ........................................ 175

Figure 19 Validation of Interpersonal criteria - Panel $6 \ldots \ldots \ldots \ldots \ldots \ldots \ldots \ldots \ldots \ldots \ldots . . .176$

Figure 20 Validation of model Alternatives - Panel 7 ................................. 177 
Figure 21 Finalized Assessment model .................................................... 180

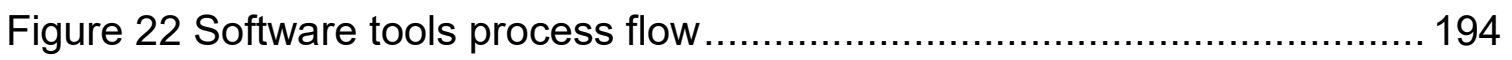

Figure 23 Relative importance of technology assessment perspectives .......... 201

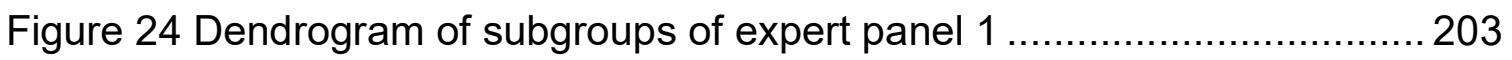

Figure 25 Relative importance of criteria in Financial perspective................... 205

Figure 26 Dendrogram of subgroups of expert panel 2 ..................................207

Figure 27 Relative importance of criteria in Technical perspective................... 210

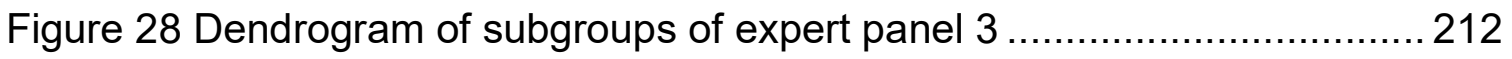

Figure 29 Relative importance of criteria in Organizational perspective ...........214

Figure 30 Dendrogram of subgroups of expert panel $4 \ldots \ldots \ldots \ldots \ldots \ldots \ldots \ldots \ldots \ldots \ldots . . .216$

Figure 31 Relative importance of criteria in Patient perspective ..................... 218

Figure 32 Dendrogram of subgroups of expert panel $5 \ldots \ldots \ldots \ldots \ldots \ldots \ldots \ldots \ldots \ldots \ldots . . .220$

Figure 33 Relative importance of criteria in Interpersonal perspective ............ 223

Figure 34 Dendrogram of subgroups of expert panel 6 .............................. 225

Figure 35 Relative importance of model alternatives with respect to criteria in

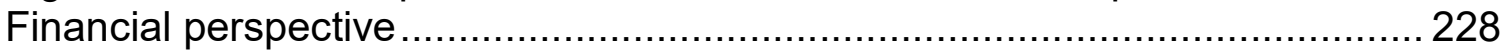

Figure 36 Relative importance of model alternatives with respect to criteria in Technical perspective.

Figure 37 Relative importance of model alternatives with respect to criteria in Organizational perspective ................................................................... 234

Figure 38 Relative importance of model alternatives with respect to criteria in Patient perspective 236

Figure 39 Relative importance of model alternatives with respect to criteria in Interpersonal perspective 238

Figure 40 Relative importance of criteria with respect to the mission 242

Figure 41 Relative importance of model alternatives with respect to Financial perspective 243 
Figure 42 Relative importance of model alternatives with respect to Technical perspective

Figure 43 Relative importance of model alternatives with respect to Organizational perspective

Figure 44 Relative importance of model alternatives with respect to Patient perspective

Figure 45 Relative importance of model alternatives with respect to Interpersonal perspective

Figure 46 Overall importance of model alternatives with respect to the mission

Figure 47 Global contribution of alternatives to the mission in scenario 1 ........ 262

Figure 48 Global contribution of alternatives to the mission in scenario 2 ........ 263

Figure 49 Global contribution of alternatives to the mission in scenario 3 ........ 264

Figure 50 Global contribution of alternatives to the mission in scenario 4 ........ 265

Figure 51 Global contribution of alternatives to the mission in scenario 5 ........ 266 


\section{CHAPTER 1. INTRODUCTION}

In the age of information and technology, rapid and meaningful assessment, diffusion and adoption of technology is the key for progressive improvements in society overall and especially in the healthcare industry. Pervasive care technologies in healthcare need to be used to their fullest potential for the improvement in healthcare quality and enhancement of clinician productivity with the ultimate goal of patient satisfaction.

Sometimes, we rarely think about how we assess technology and why do we choose to adopt one technology over the other as well as what makes us acquire it, stick with it, or trade it for a better one, and how we decide what is "better" and the aspects of a "better fit". Constant advancements in technology and information systems have to satisfy consumers and users of the system from the technical point of view and from an organizational and interpersonal viewpoint. With constant competition of information systems driving the progress of society, the need for improvements in analysis and methodologies in technology adoption is apparent and crucial.

Some of the main challenges of this century in healthcare technology assessment, mostly characterized by researcher Banta (2003), have been outlined as: 1) technology is associated with the ageing population and increased demands; 2) clinicians and researchers have produced growing evidence of poor quality of care, use of ineffective and untested technology; overuse and 
inappropriate use of technology; 3) cost-driven decision-making in policy and others.

On the positive side, the development of information and communication technologies with hopes to revolutionize the field of healthcare and provide efficient, innovative and affordable solutions is on the rise with a combination of cutting-edge technology and clinical knowledge creating a new dimension of health and social care (Banos et al. 2014). The latest report by StartUp Health listed $\$ 6$ billion in investment in digital health for 2015 with the top active markets of patient/consumer experience with a focus on prevention and savings; wellness/benefits; workflow; personalized health/quantified self; medical device; e-commerce; and big data/analytics (StartUp Health 2015).

The healthcare scene is changing through enhanced monitoring of the evolving continuum of care, emergence of mHealth (practice of medical and public health supported by mobile devices; remote health monitoring and growth of wearable sensors). Ubiquitous and pervasive healthcare, terms sometimes used interchangeably, related to encompassing healthcare services in a system providing healthcare to anyone, at any time, and anywhere by removing the restraints of time and locating and increasing the coverage and the quality of healthcare, will have significant implications for chronic disease treatments with wearable sensors being physically and technologically flexible to monitor subjects in their natural environment and provide a rich stream of information, transforming the practice of medicine (Rodgers et al. 2015; Park 2011). 
Wearable sensors provide monitoring in a natural environment with the stream of information, enriching healthcare practices and enabling better pervasive care. 


\section{CHAPTER 2. LITERATURE REVIEW}

\subsection{Technology Assessment}

The area of management of technology called technology assessment was first developed in the late 1960s in the United States and has its origins in the policy needs of U.S. Congress and for the purposes of assisting in public policy decision making. Joseph Coates in 1974 defined technology assessment as "the name for a class of policy studies which attempt to look at the widest possible scope of impacts in society of the introduction of a new technology. Its goal is to inform the policy process by putting before the decision maker an analyzed set of options, alternatives and consequences" (Coates 1974; Tran and Daim 2008). However, as the technology assessment evolved in the research and public decision making community, the meaning of technology assessment for companies differed from one of "technology readiness" in the policy setting, but was more anticipatory as far as accepting the effects of the outside world on the activities of the companies i. e. "inverted technology assessment" (Tran and Daim 2008).

Taxonomic review of methods and tools in technology assessment has been done in 2008 by researchers Tran and Daim, becoming a great reference for the TA approaches in public vs private domain. Table 1 below lists the approaches and methods review in this paper (Tran and Daim 2008). 
Table 1 Technology assessment approaches and methods in public vs private domain (Tran and Daim 2008).

\begin{tabular}{|c|c|}
\hline \multicolumn{2}{|c|}{ Technology assessment approaches and methods } \\
\hline in public domain & in private domain \\
\hline $\begin{array}{c}\text { Structural modeling and system } \\
\text { dynamics }\end{array}$ & Cost benefit methods \\
\hline Impact analysis & Decision analysis \\
\hline Scenario analysis & Measures for technology \\
\hline Risk assessment & Roadmapping \\
\hline Decision analysis & Scenarios and Delphi \\
\hline $\begin{array}{c}\text { Environmental concerns and } \\
\text { integrated technology assessment }\end{array}$ & $\begin{array}{c}\text { Surveying, information monitoring, } \\
\text { new technology }\end{array}$ \\
\hline Emerging technologies & $\begin{array}{c}\text { Mathematical and other synthesis } \\
\text { methods }\end{array}$ \\
\hline
\end{tabular}

\subsubsection{System dynamics and structural modeling}

Computer-based structural modeling techniques were studied by Linstone and Watson (Watson 1978). Those include Interpretive Structural Modeling (ISM), ELECTRE, SPIN, IMPACT, KSIM, XIMP and QSIM. ISM is a computeraided method, where for the purposes of technology assessment, a graphical representation of system composition and structure is reflected (Linstone et al. 1979). Bimodal System Dynamic Approach as a "product of two principal forces: evolutional momentum and decision-based formation" was introduced by Keller and Ledergerber (Keller and Ledergerber 1998). The process consisted of a fourstep procedure (Keller and Ledergerber 1998):

1. Description of possible application of technology, regardless of fears and desires 
2. Description of the spread and impact of individual applications under the bimodal assumption

3. Creation of hypothesis regarding the impact of decision-based alternative scenarios

4. Determining of the probable spread and impact of the individual applications according to the hypothesis and alternative scenarios

\subsubsection{Impact analysis}

Comprehensive impact assessment techniques have been described by Coates in 1974 and include 10 components according to the author (Coates 1974):

1. Problem/technology/issue or project at hand definition;

2. Definition of alternatives that need to be considered

3. Impacts identification

4. Evaluation of impacts significance

5. Identification of decision apparatus and components' responsibilities

6. Identification of decision options and alternatives

7. Identification of parties at interest/stakeholders (additional consideration)

8. Identification of macro technological alternatives (additional consideration)

9. Identification of exogenous factors (additional consideration)

10. Conclusions and recommendations. 
The research paper also described Delphi technique, trend extrapolation, morphological analysis, decision and relevance trees, economic techniques (input-output analysis, cost-benefit analysis, "at least" method, system analysis techniques, simulation, modeling, etc.

\subsubsection{Scenario analysis and Delphi}

Scenario analysis is a well-established and adopted research method particularly in the management field for the purposes of technology assessment (Tran and Daim 2008; Brent 2012; Coates 1974; Bianco et al. 2014). Coates also described scenarios as a way to systematically develop complex statements for exploring future implications [of future world states] (Coates 1974). Researchers Miller and Waller described the major strength and weaknesses of the approach, which:

1. takes a top-management as well as firm-wide perspective;

2. strives to handle the complexity of the external environment;

3. sidesteps reducing qualitative wealth into quantitative data (Miller and Waller 2003).

The major steps are identified as:

1. Framing of the issues;

2. Identification of participants and solicitation of inputs;

3. Drawing a picture of what is known;

4. Adding uncertainties to the picture; 
5. Sketching out possible paths;

6. Testing for plausibility;

7. Anticipation of interactive dynamics;

8. Formulation of strategies (Miller and Waller 2003).

Scenario analysis has been widely used in environmental and sustainability management (Liu et al. 2007; Ferng 2009; Pallottino et al. 2005); performance measurement (Buytendijk et al. 2010); energy management (Bianco et al. 2014; Zhang et al. 2012). In healthcare, however, scenario analysis is used in pandemic management (van Genugten et al. 2003); management of requirements of patients with mental health problems (Bierbooms et al. 2011).

Delphi techniques are widely adopted in many studies due to strong validity in management fields (Tran and Daim 2008). A recent study in assessing Delphi panel composition for strategic foresight aims to bring light to a controversial issue for critical reflection and revealed that there are several significant differences between two internal and external panels' evaluation, leading to varying consultation practices for different strategic purposes (Förster and von der Gracht 2013).

Meesapawong, Rezgui and Li explored adapted orientation for future innovation using a combined three-round Delphi and AHP approach applied in Thailand (Meesapawong et al. 2014). 


\subsubsection{Risk assessment}

Risk assessment of new technology investments has been researched in the 80 s and incorporated mostly financial investment criteria like payback period, return on investment for quantification of the risks and intangibles associated with high-technology investments (Hundy and Hamblin 1988).

Risk assessment of technology development has been applied in various capacities in many industries, thus, Alan Wilhite introduced a methodology for incorporating risk analysis and technology assessment in the evaluation of research and development projects for NASA (Wilhite 2004). He explained risk as composition of uncertainty and consequences and with the risk matrix and incorporation of NASA technology readiness levels (9TRLs) assessed technology risk using Analytical Hierarchical Process (AHP) (Wilhite 2004).

Risk assessment has been widely used in natural disaster preparedness and management (Cyranoski 2012) and healthcare (Linder and Sexton 2011; Ideker et al. 2011).

\subsubsection{Multi-Criteria Decision Analysis (MCDA)}

Multi-Criteria Decision Analysis (MCDA) is defined as "a set of methods and approaches to aid decision-making, where decisions are based on more than one criterion, which make explicit the impact on the decision of all the criteria applied and the relative importance attached to them" (Devlin and Sussex 2011). 
Decision analysis has been explored by the U.S. Office of Technology Assessment (OTA) and the roots of this practice are in psychology (how people decide and can they be helped to do it more effectively), engineering (construction of systems that are able to interact with decision-makers efficiently) and management science (efficient execution of decision process) (Merkhofer 1982). Decision analysis application could be broken down into some main parts:

- decomposing the decision problem into its basic elements

- quantifying each element

- applying principles of normative decision theory for identification of logically consistent alternatives (Merkhofer 1982).

Decision-focused technology assessment process has been presented by Merkhofer as a top-down process starting with problem definition, then alternatives generation, deterministic analysis, probabilistic analysis, informational analysis and policy evaluation with the loop of information acquisition and model refinement after informational analysis and deterministic one (Merkhofer 1982).

Multi-criteria decision analysis (MCDA) methods are becoming more noted in the literature since the scale and complexity in decision-making grows (Diaby et al. 2013; Goetghebeur et al. 2012; Russo and Camanho 2015). Cunningham and van der Lei introduce an approach closely related to MCDA called exchange modeling with improved prescriptions for strategy in the environment of converging technologies (Cunningham and van der Lei 2009). 
Researchers Thokala and Duenas (2012) analyzed MCDA approaches and compared them to NICE (National Institute for Health and Clinical Excellence). According to Thokala and Duenas (2012), MCDA process consists of certain stages:

1. Problem structuring (alternatives, key issues, goals, constraints, uncertainties)

2. Capturing evidence (literature review, build models, stakeholder meetings, conduct surveys)

3. MCDA modelling (define criteria, choose relevant MCDA method, performance scale values, elicit weights, aggregation)

4. Deliberation (information synthesis, sensitivity analysis, robustness analysis, challenging intuition).

Recent research advocates incorporating MCDA into HTA (Devlin and Sussex 2011). Simple linear aggregation is a common MCDA approach. In this method each score on each criterion is multiplied by its weight and then the weighted scores are summed for the overall score of that option and possibly compared with other options (Devlin and Sussex 2011).

More complex approaches include Analytic Hierarchy Process (AHP); Multi-Attribute Utility (MAU) Theory and Outranking and Data Envelopment Analysis (DEA) (Devlin and Sussex 2011; Thokala and Duenas 2012).

In healthcare, MAU is mostly used to estimate weights or 'utilities' for health states according to generic patient reported outcome measures, where the 
health states comprise different levels through different health criteria dimensions; while DEA is a technique more commonly used to measure the efficiency of hospitals (Devlin and Sussex 2011).

\subsubsection{Outranking approach}

Outranking approach is based on the general concept of dominance and utilizes outranking relation - comparison of performance score on individual criterion for the purpose of determining which alternative outranks the other on that criterion) on a set of alternatives with pairwise comparisons for estimation of concordance and discordance indices (Thokala and Duenas 2012).

ELECTRE as a method for choosing the best action(s) from a given set of actions was first proposed by Bernard Roy in 1965. The acronym ELECTRE stands for ELimination Et Choix Traduisant la REalit'e (ELimination and Choice Expressing the Reality (Figueira et al. 2005). Over the decades versions ELECTRE I (the meaningful compensation method, based on substitution rates with the ability to fix a minimum and maximum value for each one and define a set of embedded fuzzy relations), ELECTRE II (the method that deals with the problem of ranking actions from the best option to the worst), ELECTRE III (use of pseudo-criteria and fuzzy binary outranking relations) and ELECTRE IV (ranking actions without using the relative criteria importance coefficients; equipping embedded outranking relations framework) were developed (Figueira et al. 2005). 
PROMETHEE (Preference Ranking Organization METHod for Enrichment Evaluations) is another multi-criteria decision analysis (MCDA) outranking technique with the main distinction of the utilization of the generalized criterion functions with the purpose of the facilitation of inclusion of the inherent uncertainty in the criteria performance values in the process of decision analysis. General process sequence for PROMETHEE, according to Hyde et al. (2003) is the following:

1. identification of the final decision makers, actors and stakeholders;

2. criteria selection;

3. formulation of the alternatives;

4. weighting the criteria;

5. assessment of the performance of alternatives against the criteria;

6. selection of the generalized criterion function and associated indifference and preference values for each criterion;

7. applying PROMETHEE;

8. sensitivity analysis;

9. final decision.

Despite the inclusion of generalized criterion, researchers note that potential sources of considerable uncertainty remain, when PROMETHEE is utilized for a decision analysis problem, which could result in lack of consensus among the actors and diminished confidence in the decision analysis outcome (Hyde et al. 2003; Thokala and Duenas 2012). 
GAIA (Geometrical Analysis for Interactive Assistance) is a descriptive extension of the PROMETHEE methods, which provides the decision maker with a two-dimensional graphical representation of the multi-criteria problem, however, the loss of information resulting from the principal components analysis of this method can result in inconsistencies in rankings with PROMETHEE. Researchers are working on improving GAIA's limitations and overall decision making with PROMETHEE and GAIA (Hayez et al. 2009).

\subsubsection{Value measurement}

Value measurement approach represents evaluation interventions, which are based on overall benefit score, which is estimated as the weighted average of the criteria (Marsh et al. 2014). Value measurement models consist of the following steps (Thokala and Duenas 2012):

1. preference modeling - constructing the performance levels of alternatives on all criteria shown;

2. measurement of the importance of different criteria by using gain associated with replacing the worst outcome with the best outcome;

3. assigning the weights independently of the alternatives for the purposes of consistency across the comparisons with scores for each criterion being assigned to each alternative;

4. normalization of weights (interpretation of each criterion as proportion of total weight; 
5. aggregation of partial value functions, while taking into account the relative importance of different criteria.

The following methods are based on value measurement approaches: AHP, additive aggregation (weighted sum methods), MAUT, programme budgeting and marginal analysis (PBMA) (Mühlbacher and Kaczynski 2016).

New research is emerging in the area of fuzzy multiple criteria decision making, such as the study of measuring the value of patents (Wang and Hsieh 2014). The researchers use AHP and fuzzy survey after the criteria have been extracted by factor analysis.

\subsubsection{Goal programming}

Goal programming explores derivation of the alternatives that are closest to reaching the pre-defined desirable levels of achievement for each criterion (Marsh et al. 2014). Goal programming incorporates a mathematical formulation of the satisfactory heuristic (Mühlbacher and Kaczynski 2016). The satisfaction model in goal programming is directed towards achieving satisfactory levels of performance for each of the criteria according to the criteria preferences in the order of importance; and the algorithm is used to identify the alternatives that satisfy goals in a certain priority order (Mühlbacher and Kaczynski 2016). Some main examples of goal programming approaches are TOPSIS (Technique for Order of Preference by Similarity to Ideal Solution) and Data Envelopment Analysis (Thokala and Duenas 2012; Mühlbacher and Kaczynski 2016). One of the drawbacks of this method is possible significant computation time. 


\subsubsection{Cost benefit analysis methods}

Cost-benefit analysis methods are popular in economic and financial analysis literature worldwide and overall the keyword "cost-benefit analysis methods" yields 1,623,463 results in the current PSU library database. While studying assessment of technologies for obtaining advanced engineering technologies, Gagnon, focused on the discounted cost-benefit ratio and net present value (Gagnon 1991).

Recent research of measuring the effectiveness of R\&D has been based on the evaluation approach called the Technology Value Pyramid (TVP), which from bottom-to-top consists of:

1. Foundation layer: Asset value of technology - practice of R\&D Process to Support Innovation;

2. Strategy layer: Portfolio Assessment: Integration with Business;

3. Outcomes layer: Value Creation (Schwartz et al. 2011).

Through their study with application of TVP they identified top metrics by innovation game (new \& improved: standalone; pushing the envelope: integrated systems; consumer products; services) and TVP level (value creation, strategy and foundation) (Schawartz et al. 2011). The top ranking ones in outcome were financial return, gross profit, market share, projected value of pipeline, and IP management; the top five metrics in strategy: financial return, projected value of pipeline, gross profit, R\&D investment/sales and strategic alignment; and the top metrics in foundations were IP management, people development, creativity and cost versus budget. 


\subsubsection{Roadmapping}

Management of technology field has been widely employing Roadmapping as an emerging effective tool of forecasting and planning management (Tran and Daim 2008). Technology roadmapping is a powerful and flexible technique that supports technology management and strategic planning with explorations of dynamic linkages between technological resources, organizational objectives and changes in external environments as well as identifying new opportunities for achieving desired outcomes (Daim and Oliver 2008; Amer and Daim 2011; Carvalho et al. 2013).

Researchers Daim and Oliver studied the implementation of technology roadmaps at a federal agency in the areas of transmission, renewables and energy policy (Daim and Oliver 2008). The researchers identified important stages like technology gap analysis; identification of technology candidates; evaluation and prioritization of technologies; roadmapping of technologies and allocation of resources to the R\&D programs or acquisition of technologies (Daim and Oliver 2008).

A recent study by Lee, Kim and Phaal in technology roadmapping extended research in suggesting opening of communication channels to improve credibility of TRMs (Lee et al. 2012).

Geum et al. (2011) explored the application of technology roadmapping for technology-based product service integration and proposed the typological configurations of integrated roadmaps. Researchers showed the benefits of their 
application of technology roadmapping in the case of U-healthcare service - a new paradigm in medical care with ubiquitous sensor network through mapping relationships between product \& service characteristics of technology (Geum et al. 2011).

Fleischer and others describe methods used for assessing emerging technologies like Life Cycle Analysis and Materials Flow Analysis and make the case for using roadmapping for assessment of the emerging nanotechnology (Fleischer et al. 2005).

Another group of researchers used multi-level perspective (MLP) as the basis theoretical framework with the combination of methods from fields of foresight, impact assessment, simulation modeling and societal embedding (Auvinen et al. 2014). Their decision-making diagram consists of 5 main process categories (Auvinen et al. 2014):

1. Identification of the decision-making situation

2. Analysis of the socio-technical system

3. System transition roadmap: vision paths and policies

4. System dynamics modeling and simulations

5. Interpretation of the results

\subsubsection{Mathematical and synthesis methods}

One of the early examples of mathematical and synthesis methods was a study by Sharif and Sundarajan, in which they presented a quantitative model for the multi-criteria technology selection process with the inclusion of socio- 
economic and technological factors (Sharif and Sundararajan 1983). Also, researchers Grupp and Hohmeyer developed a technometric model for the assessment of technological standards and their application to selected technology-intensive products (Grupp and Hohmeyer 1986).

The use of bibliometrics and patent analysis has been researched by Daim et al. and shown in case studies of fuel cell, food safety and optical storage technologies (Daim et al. 2006).

The use of structural modeling (SM) techniques has been analyzed and applied already in 1979 by Linstone and others looking at seven specific methods involving diagrams and matrices, specifically: Interpretive Structural Modeling (ISM); ELECTRE - computerized algorithm for ranking a set of alternatives; computerized structuring tool called SPIN; IMPACT; KSIM, XIMP and QSIM (Linstone et al. 1979).

Shen et al. proposed a technology selection process integrating fuzzy Delphi method, analytic hierarchy process and patent co-citation approach (PCA) in the case of selection of the organic light emitting diode (OLED) technology in Taiwan (Shen et al. 2010). 


\subsection{Healthcare Technology Assessment}

\subsubsection{History and main objectives of HTA}

Healthcare Technology Assessment (HTA) has been developing as a field for a number of decades, blooming around addressing questions concerning technology, benefits, costs and quality of care. In the United States, the historical beginnings of healthcare technology assessment could be traced to 1975 - to the establishment of a health program in the Congressional Office of Technology Assessment (OTA) (Battista et al. 1994). In 1994, David Banta analyzed eight industrialized countries: Australia, Canada, France, Germany, the Netherlands, Sweden, the United Kingdom and the United States in order to learn about technology assessment in their management of healthcare (Banta 1994). He noted the advancements in basic research in healthcare, producing new knowledge about biological mechanisms of the human body and its malfunctions in disease, however, Banta stressed that paths of technology development and interventions in basic research have not been satisfactory as a policy tool.

Researchers Jonsson and Banta analyzed the Swedish healthcare system and gave high remarks to the health technology assessment in that country, since technology assessments performed by the Swedish Council of Technology Assessment in Health Care (SBU) are not just clinical or outcome assessments, but also "analyses of the nature of particular problems in Swedish society and evaluations of context and technology from diverse standpoints (including social 
and economic)" (Jonsson and Banta 1994). There, technology assessment was introduced with two main objectives:

1) to speed the diffusion and use of medical technologies with proven safety, efficacy and effectiveness in order to ensure broad and even access to the technology;

2) to monitor the not-yet-assessed technologies whose policy implications are not yet understood in order to replacement and/or phasing out of useless, less effective or potentially harmful technologies (Jonsson and Banta 1994).

\subsubsection{Problems in technology diffusion in healthcare}

The diffusion of new technology in healthcare culminates with the attainment of an appropriate level of use for the technology or with abandonment of it, since if the new technology appears to be of value, clinician's use of it gets transferred onto patients, who become familiar with it and may ask for it (Banta 1994).

Banta presents various case studies discussing the way each country dealt with the technologies, benefits and costs and revealed the forces at work in the process of technological diffusion (Banta 1994). He also did a number of exploratory studies of technology assessment on technologies like minimally invasive therapy (MIT), which is based on developments of endoscopy, vascular catheters, imaging devices, and related technologies (Banta 1993). In the other paper on MIT, Banta, Schersten and Jonsson stress that complexities of new 
technologies and procedures associated with them there is a need for quality assurance, set protocols, collection and analysis of data with the goals of care being explicitly spelled out (Banta et al. 1993). According to the researchers, data system with example of minimally invasive therapies must include:

- information about the operation (or therapy);

- information on nursing after the procedure;

- information about home care (follow-up);

- information on complications

- information on satisfaction/patient complains

- feedback loop to encourage evaluation and learning (Banta et al. 1993). Communication is essential to assure proper care and follow-up and new means of communication and additional training, introduction of alternatives and full information on benefits, risks and costs need to be implemented in a system to ensure correct output to the system and outcome (Banta et al. 1993). Policies towards medical technologies may appear in various forms depending on the organizations that apply them (from research institutions, medical facilities, management to TA organizations and mass media) and include experiments, publications, education and training, planning, budgets, investment control, certifications, payment, evaluation (technical assessment) and consumer information (Banta et al. 1993).

In a different paper on the assessment of technology with outlined procedures, the author states that the effectiveness of any new procedure should 
be established by well-controlled clinical trials and the evaluation of that effectiveness has to be a part of the diffusion process of any procedure and finally the effective, or better even - cost-effective procedure may be a part of the healthcare system (Banta 1993). The researcher concludes that the lack of an evaluation strategy in healthcare stunts the development of cost-effective innovation in medical practices (Banta 1993).

Looking at the technology diffusion in selected cases, the reality of it demonstrates that with the uncertainties of effectiveness or cost-effectiveness of technology, the diffusion of it matters on the grounds of encouraging or discouraging it (Banta and Vondeling 1993). The researchers apply a simple model to the data available and perform certain steps:

1. Assessment of the current diffusion pattern of each case and mapping it to three situations:

a. hardly diffused in the healthcare system;

b. diffused slow;

c. rapidly diffused

2. Judgment on the desirability of additional diffusion of each case (Banta and Vondeling 1993).

Out of the main factors supporting diffusion of technology in healthcare, the researchers outline the payment system and importance of financing, patient demand and physician interest (Banta and Vondeling 1993). 


\subsubsection{Main steps in HTA and impacts on technology implementation}

A group of researchers analyzed technological fields for home health care application. Those included: micro-electronics/informatics, biotechnology/molecular biology, and biomaterials (van Beekum and Banta 1989). They also emphasized several important axioms about technology implementation:

- systemized approach;

- needs-oriented;

- provided when appropriate;

- paid for;

- evaluated (van Beekum and Banta 1989).

While commenting on priorities that have been developed for healthcare technology assessment, Banta wrote that setting priorities is critical for success of the field since the goal of technology assessment is to stimulate rapid changes in health care, mostly through health policies; and the lack of priority-setting methods and activities could explain the limiting impact (Banta 1991). Another interesting paper on identification and priority setting for HTA in the Netherlands shows that methods are not very transparent and methods for setting priorities on the basis of societal criteria so developed (Oortwijn et al. 1999).

An earlier paper by Banta, Gelijns, Griffioen and Graafs identified a number of future categories of technologies:

1. disease prevention and health promotion; 
2. nutrition;

3. the brain, behavior, and mental health;

4. reproductive health;

5. fetal and child health;

6. medical imaging and other diagnostic technologies;

7. biotechnology-related developments;

8. biologics and pharmaceuticals;

9. blood banking;

10. artificial and transplanted organs and tissues;

11. laser technology;

12. other progress in therapy;

13. rehabilitation and home care;

14. oral health;

15. alternative therapy;

16. clinical application (information technology);

17. organizational and analytical applications (information technology);

18. basic and applied sciences (Banta et al. 1987).

In the same article, Banta outlined the four steps of the healthcare technology assessment:

1. Identification and selection of the specific technology;

2. Collection of data to the extent possible;

3. Synthesis of the data and information from the literature; 
4. Dissemination of the information (Banta et al. 1987).

\subsubsection{Use of scenarios in healthcare technology assessment}

Banta et al. (1987) describes the analysis of the 30 identified technologies on the basis of the following criteria:

- impact on future health needs;

- long-range health care system impacts;

- expected financial consequences;

- potential social and ethical implications (Banta et al. 1987).

The researchers used expert surveys and scenarios for a number of technology assessments since this method "combines qualitative and quantitative information and attempts to discuss an alternative future", however in the case of the study of lasers, system dynamic modeling techniques were employed to develop more quantitative scenarios (Banta et al. 1987). The conclusions about the development of technology forecasting as a valuable instrument for policy and decision-making has been made with organization of a comprehensive technology assessment system directed at the different stages of the life cycle of a particular technology or set of technologies:

- future technological applications that are in R\&D stage;

- emerging technology prior to adoption;

- new technology in the phase of adoption; 
- accepted technology in general use;

- obsolete technology that needs to be taken out of use (Banta et al. 1987).

\subsubsection{Healthcare assessments based on outcome studies}

Banta and Bos (1991) researched the relationships between quality and quantity with coronary artery bypass graft $(C A B G)$ surgery with literature reviews of outcome studies and plotting the number of studies inconsistent with hypothesized volume-outcome relationship vs. number of studies showing worse outcomes at lower volumes. The authors identify that evidence-based studies fail to identify hospital factors other than volume that account for a better outcome and factors like the importance of the entire team in open-heart surgery or patient mix have been difficult to define and measure (Banta and Bos 1991). In another paper, Banta stresses the factors of safety and efficacy in medical device technologies noting of the weak systems for such assurance in most European countries (Banta 1988b).

\subsubsection{Decision-making methodologies in healthcare}

In the fields of Healthcare Analytics and Informatics, the major areas of concern, where decision-making has been pursued is in the areas of Clinical Decision Support and Quality/Performance Improvement (Greenes 2007; Strome 2013; Möller et al. 2011; Rifat 2013). 
Primary research methodologies have been described in Table 2

(Greenes 2007) below:

Table 2 Primary research methodologies in the areas of Clinical Decision

Support and Quality/Performance Improvement

\begin{tabular}{|l|l|l|}
\hline Methodology & Major Uses & Key developments \\
\hline Information retrieval & $\begin{array}{l}\text { Finding information } \\
\text { answering questions }\end{array}$ & $\begin{array}{l}\text { Taxonomies, ontologies, } \\
\text { text-based methods, } \\
\text { patient-specific context } \\
\text { keys, automatic } \\
\text { invocation }\end{array}$ \\
\hline $\begin{array}{l}\text { Evaluation of logical } \\
\text { conditions }\end{array}$ & $\begin{array}{l}\text { Alerts, reminders, } \\
\text { constraints, inferencing } \\
\text { systems }\end{array}$ & $\begin{array}{l}\text { decision tables, event- } \\
\text { condition-action rules, } \\
\text { production rules }\end{array}$ \\
\hline $\begin{array}{l}\text { Probabilistic and data- } \\
\text { driven classification or } \\
\text { prediction }\end{array}$ & $\begin{array}{l}\text { Diagnosis, technology } \\
\text { assessment, treatment } \\
\text { selection, classification } \\
\text { and prediction, prognosis } \\
\text { estimation, evidence- } \\
\text { based medicine }\end{array}$ & $\begin{array}{l}\text { Bayes theorem, decision } \\
\text { theory, ROC analysis, } \\
\text { data mining, logistic } \\
\text { regression, artificial } \\
\text { neural networks, belief } \\
\text { networks, meta-analysis }\end{array}$ \\
\hline $\begin{array}{l}\text { Heuristic modeling and } \\
\text { expert systems }\end{array}$ & $\begin{array}{l}\text { Diagnostic and } \\
\text { therapeutic reasoning, } \\
\text { capturing nuances of } \\
\text { human expertise }\end{array}$ & $\begin{array}{l}\text { Rule-based systems, } \\
\text { frame-based reasoning }\end{array}$ \\
\hline $\begin{array}{l}\text { Calculations, algorithms, } \\
\text { and multistep processes }\end{array}$ & $\begin{array}{l}\text { Execution of } \\
\text { computational } \\
\text { processes; flow-chart- } \\
\text { based guidelines and } \\
\text { consultations, interactive } \\
\text { dialogue control, } \\
\text { biomedical image and } \\
\text { signal processing }\end{array}$ & $\begin{array}{l}\text { Process flow and } \\
\text { workflow modeling, } \\
\text { guideline formalisms and } \\
\text { modeling languages }\end{array}$ \\
& $\begin{array}{l}\text { Structured data entry, } \\
\text { structured reports, order } \\
\text { sets, other specialized } \\
\text { presentations and data } \\
\text { views }\end{array}$ & $\begin{array}{l}\text { Report generators and } \\
\text { document construction } \\
\text { tools, document } \\
\text { architectures, templates, } \\
\text { markup languages, } \\
\text { ontology tools, ontology } \\
\text { languages }\end{array}$ \\
& \multicolumn{2}{|l}{} \\
\hline Associative groupings of \\
elements
\end{tabular}

Let's explore related research methodologies in more detail below. 


\subsubsection{Decision tables}

Decision tables are used when logic needs to be used in order to refine or reduce the number of possibilities, most likely diagnostic possibilities in healthcare. For example, the table could be constructed with the values of individual findings $\left(f_{i}\right)$ as positive, negative or unspecified i.e. 1,2 or "-" for disease $D_{i}$ (Greenes 2007). The finding values and their combinations could be sorted and manipulated for decision-making purposes. The advantage of decision tables could be described in their ability to sort and group columns with similar values in their rows. They also could be improved by including columns with certain combinations of findings and rows of actions. Introduction of probabilistic and logic manipulation in healthcare was pioneered by Ledley and Lusted with their classic Science article entitled "Reasoning Foundations of Medical Diagnosis" (Ledley and Lusted 1959).

\subsubsection{Decision analysis}

The methodology of statistical decision theory was pioneered by Howard Raiffa and Ronald Howard (Raiffa 1997; Howard 1966; Howard 2006). Application of the methods in clinical decision making and formal decisionmaking analysis spread in the 70s (Schwartz et al. 1973; Pauker 1976). The sequences of decisions and possible outcomes at each step are depicted in a decision tree, so that the focus of the decision maker would be on the critical variables (Greenes 2007). Upon expansion of the branches of a decision tree, 
endpoints could be assessed by assigning utilities to them. From a chance node, the branches are assigned conditional probabilities and the solution proceeds by a method known as fold-back analysis. The optimal decision is considered in general to be one that maximizes Expected Utility (EU). Sensitivity analysis on the various estimated parameters to examine robustness of the decision over reasonable ranges of key parameters and for purposes of determining of a parameter threshold (Pauker and Kassirer 1987) is performed.

\subsubsection{Bayesian belief networks}

The notion of Bayesian belief networks that depict the various dependencies in the form of an acyclic directed graph has been developed in the 1970s by Pearl at Stanford (Pearl 1988). Cooper applied it in the 1980s to medical problems (Cooper 1986). However, Bayesian networks have not grown in popularity through medical community and this methodology is used mostly for research purposes due to the need of severe model simplifications for practical and clinical use (Matheny and Ohno-Machado 2007).

\subsubsection{Other technology assessment techniques}

Receiver operating curve (ROC) analysis could be related to cost-benefit analysis of diagnostic decision making and provides tools to select optimal models and to discard suboptimal ones through plotting the true positive rate against the false positive rate at various threshold settings (Metz 1978; Swets 1979). Metz characterized ROC curve as a simple and yet complete description 
of the decision threshold indicating all possible combinations of the relative frequencies of the various kinds of correct and incorrect decisions (Metz 1978).

\subsubsection{Database prediction: data mining and machine learning}

Analysis of responses of patients to various treatments that are similar to an existing patient in large databases with well-structured data may allow for better decisions in treatment for that patient. Interest in database prediction has been sparked due to growth in sizes and numbers of data variables in data repositories and advances in molecular biology and genomics (Greenes 2007).

The power of the database prediction has been demonstrated by Bruce ad Fries in a case of chronic disease databank ARAMIS, which has enabled the study of real-world patient-reported outcomes (PROs) in rheumatology and resulting improved approaches to treatments, cost effectiveness, outcome and quality of life (Bruce and Fries 2005). Regression and nearest neighbor techniques as well as more recent non-linear techniques and the use of fuzzy logic have been refined and are the main ones used in database prediction field (Greenes 2007). Knowledge discovery from databases (KDD) has been stimulated by artificial intelligence (Al) techniques like artificial neural networks (ANNs) (Greenes 2007). One of the fathers of Healthcare Analytics, Trevor Strome, outlined the examples of Machine Learning in the following Table 3 (Strome 2013). 
Table 3 Machine learning techniques in Healthcare Analytics.

\begin{tabular}{|c|l|}
\hline $\begin{array}{c}\text { Machine learning } \\
\text { system }\end{array}$ & \multicolumn{1}{c|}{ Description } \\
\hline \multirow{2}{*}{$\begin{array}{c}\text { Artificial neural network } \\
\text { (ANN) }\end{array}$} & $\begin{array}{l}\text { ANN is biologically inspired mathematical model } \\
\text { based on our understanding of how neurons in the } \\
\text { brain function. The pattern recognition capabilities } \\
\text { of ANN mimic those found in biologic systems } \\
\text { (Brown 2008). The algorithm of ANN is } \\
\text { characterized with robustness, high accuracy and } \\
\text { output of discrete or real values. }\end{array}$ \\
\hline Decision tree & $\begin{array}{l}\text { Resembling a game of "20 questions", in which } \\
\text { the sequence of questions is visualized by "a } \\
\text { tree": the first [root] node positioned at the top is } \\
\text { connected by successive (directional) links or } \\
\text { branches to other nodes (Duda et al. 2001). } \\
\text { The advantage of decision trees results in ease of } \\
\text { understanding and implementation in source } \\
\text { systems and business intelligence software } \\
\text { supporting basic computer operations. }\end{array}$ \\
\hline $\begin{array}{c}\text { Support vector } \\
\text { machine (SVM) }\end{array}$ & $\begin{array}{l}\text { SVM is used for both linear and nonlinear types of } \\
\text { data and works by separating entities into mutually } \\
\text { exclusive regions (Hastie et al. 2009). A few points } \\
\text { along the boundary area (the support vectors) } \\
\text { start the process, then the data is transformed into } \\
\text { new spaces, where the separation between the } \\
\text { classes gets improved. }\end{array}$ \\
\hline
\end{tabular}




\subsubsection{Applications of hierarchical decision modeling in healthcare}

When we are trying to select a product or technology based on a number of alternatives, we engage in a decision-making process. While we make our decisions every day, some of them are more complex than the routine kind and require established managerial methodologies created for this purpose. Hierarchical Decision Model (HDM) is used to decompose the problem into hierarchical levels and using pairwise comparison scales and judgment quantification technique, the researcher arrives at the calculated alternative. However, the process of decision analysis is even more of a value than the answer it brings, since it forces systematic assessment of the alternatives (Henriksen 1997). Decision analysis provides information, so that managers of technology, in this case, healthcare information technology, can make more informed decisions.

Community-wide implementation of health information technology was studied by Goroll et al., thus, Massachusetts eHealth Collaborative (MAeHC) was formed in order to improve patient safety and quality of care through HIT use promotion (Goroll et al. 2008). The working group outlined a set of system features (user friendliness, functionality, clinical decision support capability, interoperability, security, reliability and affordability) that were involved in selection of vendors (Goroll et al. 2008). The authors also stress that despite the national push of HIT implementation, positive encouragements in terms of vendor certification and system standards, the current state of standards cannot ensure 
sufficient specific fit for a routine use by practices, interoperability and ease of use, therefore considerable technical as well as organizational efforts need to be engaged in the system (Goroll et al. 2008).

Some interesting examples of hierarchical decision models in healthcare were described by Bohanec and others (Bohanec 2000), and were clinical in nature (assessment of breast cancer risk, assessment of basic living activities in community nursing, risk assessments in diabetic foot care etc.), using DEX, an expert system shell for multi-attribute decision support.

\subsubsection{HTA in the United States compared to other countries}

While analyzing papers by Battista and others, it could be noted that some unique traits of the system in the United States consist of:

- In healthcare system: multiple payers; Medicaid/care public financing, corporate roles and interests, administratively cumbersome and increasing reform pressure

- In regulation of drugs: FDA; large domestic industry; proposals to speed up the process when applicants support costs of regulatory requirement

- In regulation of equipment: classes I, II, and II are established by law with exemptions for devices that are 'substantially equivalent'; certificate-of need programs 
- In regulation of physicians: Fee-for-service practices with increasing amount of 'managed care'; imbalance among the number of specialists vs. the number of generalists

- In research and development: large industry with extensive R\&D; high level of government funds (NIH, AHCPR)

- In Technology Assessment: diverse groups with little coordination; OTA, OHTA, AHCPR, professional organizations, state-level involvement (Battista et al. 1994).

Researchers Battista, Banta and others state that while the influence of technology assessment should not be overstated, only small amount of existing technologies got formally assessed and while adoption and use of healthcare technology is influenced by many factors like perception and experience of health and disease, cultural responses, industrial, financial and regulatory systems, physicians and hospitals retain considerable autonomy in their decision-making and those decisions are made primarily in the purchasing departments of hospitals, clinics and physician practices (Battista et al. 1994).

The researchers also outlined key themes of HTA:

1. Effective links of technology assessment to technology management are essential. (Example: United States and German healthcare systems have limited policy structure to technology 
management and therefore do not effectively deal with implementing technology assessment findings).

2. The scope and impact of the technology assessment activities in healthcare system are dictated by the level of those activities (Example: Insurers in the United States pursue the forms of technology assessment with a view of regulating care providers practices to their insured clients).

3. The use of healthcare technologies is either unknown or uncertain in need of much collaboration, since the information should benefit payers, providers and mainly patients (Battista et al. 1994).

The problem of healthcare technology assessment varies in different countries, for example, in China due to the insufficient attention to fundamental issues concerning technology, its risks, benefits and costs, lack of policy, the aggressive implementation of Western technology created a system of specialized hospitals and clinics lacking needed supporting infrastructure (Banta 1990). In another paper, researchers compared evaluation and diffusion of excimer laser treatment of myopia between the United States and the Netherlands and stressed that technology assessment needs to be more international (Vondeling et al. 1995). In a letter of a response to the book called "Technology Assessment and New Kidney Stone Treatment Options" by Kamper -Jorgensen and other eds., Banta noted that clearly "health technology assessment may have little to do with clinical decisions" as well as healthcare 
technology assessment should be more effective internationally and "much needs to be done before adequate information to guide decision-making will be available to clinicians and policy-makers" (Banta 1988a). In his paper on development of health technology assessment, he defines HTA as such: "HTA seeks to couple evidence with decision-making, and thus has similarities to evidence-based health care and evidence-based policy-making" (Banta 2003).

The requirements for the successful assessments according to the successful SBU studies are the following:

1) Assessments have to result from a strong policy-maker and/or clinician interest.

2) Data on technology needs to be available from methodologically rigorous studies and randomized trials.

3) All related studies must be identified and thoroughly reviewed with the involvement of expert professionals in order to preserve integrity.

4) Scientifically and clinically credible and logically-sound assessments are a must.

5) Assessments should be presented in a way that they are accessible to the medical professionals, policy-makers and public.

6) Results need to be supplemented by specific policy options or recommendations. 
7) A strong marketing strategy on different fronts needs to go along with the results.

\subsection{Health Information Technology Assessment and Adoption}

\subsubsection{Theoretical approaches the conceptualize interaction of technology,} humans and organization

Below are some figures depicting the bodies of knowledge surrounding organizational issues in HIT innovation (Figure 1) and theoretical approaches that conceptualize interaction between technology, humans and organizations (Cresswell and Sheikh 2012) (Table 4). 


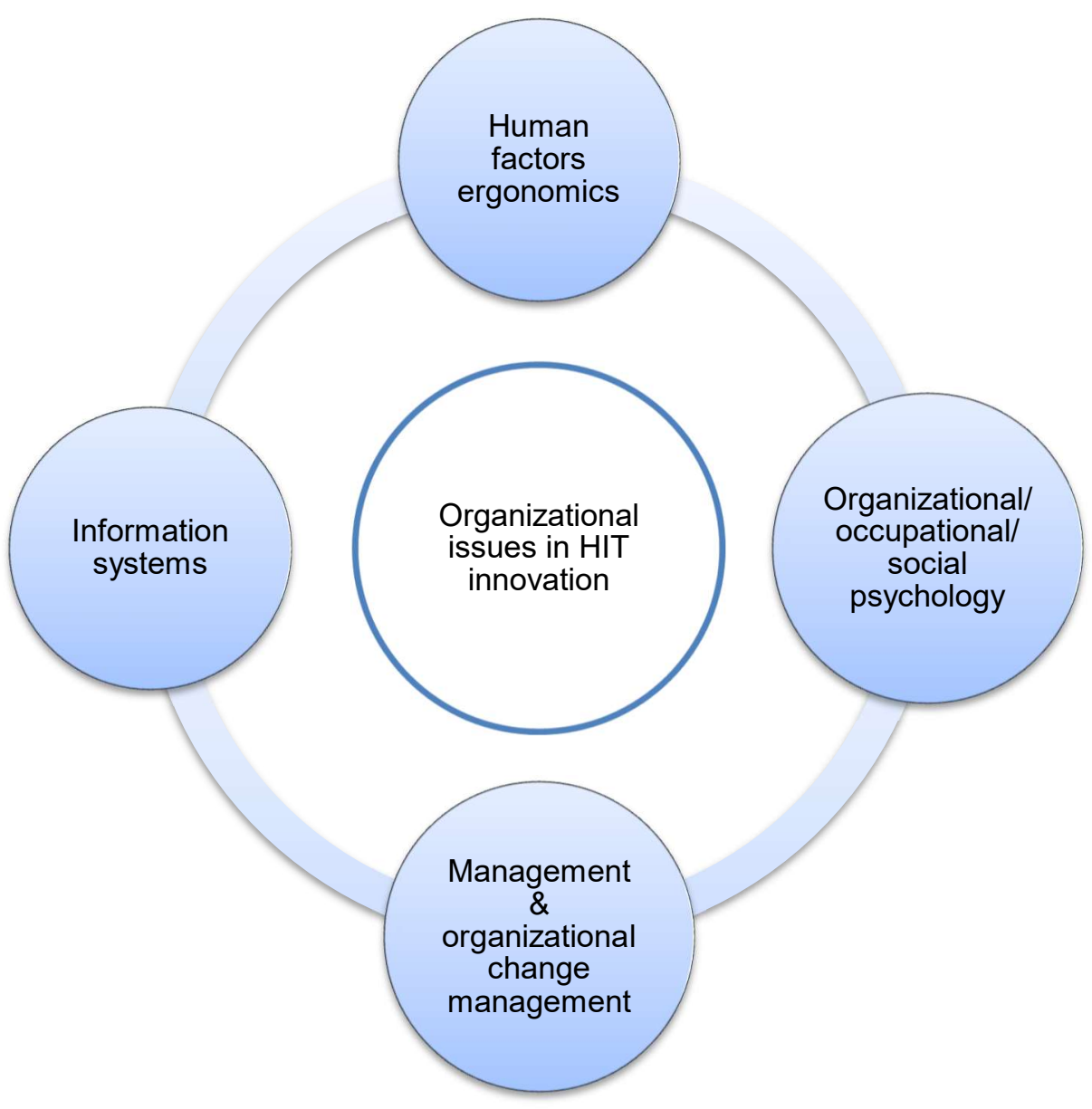

Figure 1 Bodies of knowledge surrounding organizational issues in HIT innovation.

Below is the table of theoretical approaches that conceptualize interaction between technology, humans and organizations (Cresswell and Sheikh 2012). 
Table 4 Theoretical approaches of interaction between technology humans and organizations

\begin{tabular}{|c|l|}
\hline Name of the Theory & \multicolumn{1}{|c|}{ Explanations and definitions } \\
\hline Diffusion of Innovations & \multicolumn{1}{|c|}{$\begin{array}{c}\text { Focuses on how innovations spread in and } \\
\text { across organization over time }\end{array}$} \\
\hline Normalization process & $\begin{array}{l}\text { Describes the incorporation of complex } \\
\text { interventions in healthcare into the day-to-day } \\
\text { work of healthcare staff }\end{array}$ \\
\hline Sensemaking & $\begin{array}{l}\text { Assumes that organizations are not existing } \\
\text { entities as such, but produced by sensemaking } \\
\text { activities and vice versa; they discover meaning } \\
\text { of the status quo often by transforming } \\
\text { situations into words and displaying a resulting } \\
\text { action as a consequence. }\end{array}$ \\
\hline Social shaping theory & $\begin{array}{l}\text { Views technology as being shaped by social } \\
\text { processes and highlights the importance of } \\
\text { wider macro-environmental factors in influencing } \\
\text { technology }\end{array}$ \\
\hline Sociotechnical changing & $\begin{array}{l}\text { Conceptualizes change as a non-linear, } \\
\text { unpredictable and context dependent process, } \\
\text { assuming that social and technical dimensions } \\
\text { shape each other in a complex and evolving } \\
\text { environment over time }\end{array}$ \\
\hline Technology Acceptance \\
Model & $\begin{array}{l}\text { Assumes that individual's adoption and usage of } \\
\text { the system is shaped by the attitude toward use, } \\
\text { perceived ease of use and perceived usefulness }\end{array}$ \\
\hline $\begin{array}{l}\text { Accentuates that social, technological and work } \\
\text { process factors should not be considered in } \\
\text { isolation but in the appropriate alignment with } \\
\text { each other }\end{array}$ \\
\hline
\end{tabular}




\subsubsection{Statistics of HIT adoption and diffusion}

Some of the most noticeable barriers to implementation of health information technology adoption are:

- poor product availability in accordance to the needs of the customer (18.2\% of organizations and $20.8 \%$ of clinicians);

- training requirements ( $26 \%$ of organizations and $31 \%$ of clinicians);

- costs of purchase ( $80.2 \%$ of organizations and $84.1 \%$ of clinicians) and costs of implementation (58.6\% of organizations and $68.4 \%$ of clinicians);

- inadequate ROI (36.1\% of organizations and $29.8 \%$ of clinicians) (Witter 2009).

Some other important characteristics were also privacy and security issues, lack of leading expertise, concerns about product failures and size of practice (Witter 2009).

While these concerns were surveyed in regards to EHR, a type of information technology currently being implemented throughout the United States, those problems resonate as general technology implementation concerns. Those issues call for the necessity of multi-dimensional assessment model of health technology adoption, which would enable physicians and administrators to look at the multiple perspectives of the problem, including goals, objectives and strategies of the healthcare unit, analyze those through their judgments and make their decision towards a particular information technology from all of the possible available alternatives. 
With the rising costs of healthcare, it is crucial to make sure that health information technologies are used to their fullest potential and that they would improve health care quality, clinician productivity and patient satisfaction. While the encouragement to implement computerized registries (Bufalino et al. 2011) as well as putting installation deadlines from the federal government (Blumenthal 2009) may speed up the process of registry implementation, the use of a registry by physicians and the process of integrating it in their offices that ultimately translates to an improvement in health care quality could be a difficult step to achieve (DeLia et al. 2004; Kumar and Aldrich 2010). Ford et al. (2006) predicts that electronic health record (EHR) adoption, which they classify as "general purpose" technologies that do not deliver productivity gains immediately upon arrival, will reach a peak diffusion in 2024 in the small practice settings (Ford et al. 2006; Ash and Bates 2005).

Large hospitals have been early adopters of computerized registries (Palacio et al. 2009; Yoon-Flannery et al. 2008; Zandieh et al. 2008), electronic health records and electronic medical records, while small primary care practices, disadvantaged groups, family practices and other ambulatory clinics have been shown to be slow-adopters of health IT (Lustria et al. 2011).

In its recent study and Report to the President, The President's Council of Advisors on Science and Technology (PCAST), noted that nearly 80 percent of physicians, primarily small and independent practices, are lacking digital health records. United States is behind in adoption of health records compared to other 
industrialized nations (Schoen et al. 2006). Some of the main problems in adoption of health records, mentioned in the report include limited functionality and poor interoperability (Alper and Olson 2010).

\subsubsection{Barriers to adoption in health information technology studies}

\subsubsection{Main research studies}

One significant study with a systematical literature review of perceived barriers was done by Albert Boonstra and Manda Broekhuis. The authors identified 8 categories: financial; technical; time; psychological; social; legal; organizational and change process (Boonstra and Broekhuis 2010). The first three categories were identified as the primary categories. The below Table 5 summarizes the taxonomy of the "primary" barriers: 
Table 5 Taxonomy of "primary" barriers (adopted from Boonstra and Broekhuis 2010).

\begin{tabular}{|l|l|}
\hline Category & Barrier \\
\hline Financial & $\begin{array}{l}\text { High start-up costs } \\
\text { High ongoing costs } \\
\text { Uncertainty about ROI (return on investment) } \\
\text { Lack of financial resources }\end{array}$ \\
\hline Technical & $\begin{array}{l}\text { Lack of computer skills of the physicians and/or the staff } \\
\text { Lack of technical training and support } \\
\text { Complexity of the system } \\
\text { Limitation of the system } \\
\text { Lack of Customizability } \\
\text { Lack of Reliability } \\
\text { Interconnectivity/Standardization } \\
\text { Lack of computers/hardware }\end{array}$ \\
\hline Time & $\begin{array}{l}\text { Time to select, purchase and implement the system } \\
\text { Time to learn the system } \\
\text { Time to enter data } \\
\text { More time per patient } \\
\text { Time to convert the records }\end{array}$ \\
\hline
\end{tabular}

Boonstra and Broekhuis also outlined a list of perceived barriers and the related possible interventions (Boonstra and Broekhuis 2010). Greenhalgh et al. (2009) gave a comprehensive historic literature review into tensions and paradoxes of electronic patient record research, listing barriers as well as drawbacks of adoption.

In another recent study by Lorenzi et al., the authors stress that scale of U.S. ambulatory practices is an important differentiator in the way the information technology is implemented and outline some barriers, and potential benefits (improved patient care, improved office efficiency, potential financial benefits) of EHR adoption (Lorenzi et al. 2009). 
Bates et al. (2003) outlined a similar list of barriers that are noted by other research literature: Boonstra and Broekhuis (2010) and Lorenzi et al. (2009), looking at both financial and social aspects as well as stressing the risks of failure to adopt health information technology. Security concerns were also described in a study by (Lorence and Churchill 2005).

\subsubsection{Communication \& social aspect barriers}

Another important aspect that needs to be considered is communication. Thus, researchers Lanham, Leykum and McDaniel looked at the within-practice communication patterns through interview and observation data and emphasized the importance of cohesive communication for technology adoption (Lanham et at. 2012). Kai Zheng, along with the group of researchers from the East Coast universities studied how social interactions influence physician adoption of an EHR system. They found that perceived influence network and professional network are not correlated with EHR usage, while the structure of friendship network significantly influenced EHR adoption by physicians (Zheng et al. 2010).

Community-wide implementation study of HIT was done in Boston, which indicated several major barriers like inadequate standards for data representation and vocabulary, which is a tremendous impediment to data sharing and interoperability and therefore adequate communication throughout the system (Goroll et al. 2008). Another study by Hahn from New Jersey stresses that EMR usage may not lead to improved quality in a community practice setting (Hahn et al. 2011). 
One recent study by Behkami showed findings that $\mathrm{PCMH}$ barriers were not significant in their impact of practice payer mix, registry implementation and registry use (Behkami 2012). Other barriers summarized by Behkami (2012) and identified by various researchers were excessive cost to purchase HIT; lack of funds availability; increased labor; system compatibility; value misperception of technology; management and implementation complexity; training needed; workflow redesign; lack of user support (Behkami 2012; Cherry 2006; Shields et al. 2007; Kazley and Ozkan 2007; Meade and Islam 2006; Lorence and Chirchill 2005; Greenhalgh et al. 2008; Ash et al. 2001).

Another interesting framework that could potentially serve as a research model comes from HITECH (Health Information Technology for Economic and Clinical Health) Act and connects the enablers of adoption (like regional extension centers, workforce training), meaningful use (Medicare and Medicaid incentives) and exchange of health information (state grants, standards and certification framework, privacy and security) with the targets of improved individual and population health outcomes, increased transparency and efficiency and improved ability to study and improve care delivery (Blumenthal 2010). 


\subsubsection{Health IT Adoption benefits}

Integration of technology in

the the flow of clinical

practice
- Minimize unproductive data entry work
Real-time access to complete patient's data and information support
- Provide best decisions in healthcare
Integration of patient's

involvement in their own care
- Bring possibility of proactive decisions

Figure 2 Causal diagram of HIT adoption benefits

Proper implementation of Health Information Technologies could bring out the benefits of HIT illustrated in Figure 2 (Alper and Olson 2010). The authors describe a so-called "network effect" -- i.e. effect of user externality, where the value to the users increases the more they use a network -- that has been known to be a transformational effect of IT in various industry sectors. New products in such cases spur the unification of fragmented systems. Unfortunately, we haven't observed such network effects in healthcare IT. Most health IT applications are stand-alone proprietary applications that are not directly exchangeable, not easily searchable, indexed, viewed as purely internal sources and not easily adopted into a workday of a physician or a patient (Alper and Olson 2010). Some wellknown adopters of Health IT: Veteran's Health Care Administration and Kaiser Permanente are few and limited to drive the overall market adoption of healthcare IT (Box et al. 2010; Chumbler et al. 2011). 
The below Table 6 shows aggregate problems/barriers in adoption of healthcare IT described in the literature (Alper and Olson 2010):

Table 6 Problems/barriers in HIT adoption.

\begin{tabular}{|ll|}
\hline & Problems/Barriers \\
\hline - & proprietary applications \\
\hline - & not intuitive (not easily adopted into workflow of a clinician's day) \\
\hline - & not directly exchangeable data formats \\
\hline - & search ability issues \\
\hline - & context for individual entries is implicit \\
\hline - & intended for internal-only use \\
\hline - little incentive for investment in secondary or external uses \\
\hline - patient concerns of privacy \\
\hline - patient concerns of security \\
\hline - oriented toward administrative functions not better care \\
\hline
\end{tabular}

Some of those barriers were described since 1990s, like delayed rewards, clinician typing, maintenance of data, dual charting, as well as data security (Rind and Safran 1993).

\subsubsection{Financial barriers and benefits}

Several authors describe financial barriers as some of the most important ones that physicians consider while assessing health information systems (Ackermann 2011; Simon et al. 2007; Simon et al. 2008; Fonkych and Taylor 2005; Behkami 2012; Boonstra and Broekhuis 2010; Bates et al. 2003; DePhillips 2007; Rosemann et al. 2010; Shen and Ginn 2012; Valdes et al. 2004;

Vishwanath and Scamurra 2007; Yoon-Flannery et al. 2008). It could be anything from challenges of measuring ROI, seeing the direct benefits of investments, assessing overall value, or investments in extra capabilities. 
Lack of cross-training, inadequate resources and staff turnover were discovered as major threats to the registries in a study of adoption and implementation of mandated diabetes registries by community health centers (Helfrich et al. 2007).

Benefits to electronic healthcare implementation were described in a study particular to primary care (Bodenheimer and Grumbach 2003). While the researchers agree with previously described in barriers, some of those, like computer interference with patient-physician relationship, could facilitate possible solutions of intermediary services or administrative assistants. The authors believe that the benefits from the so called "electronic revolution" will be more prominent if care practices will redesign their clinical processes to ensure ehealth facilitates the work of physicians. This article touches on the aspects of quality of web-based medical information and the possibility of it being a barrier as opposed to the benefit (Bodenheimer and Grumbach 2003). Another study on practice-based innovations and factors that influence adoption noted a positive association between organizational size, organizational relationships and stakeholder expectations on the level of innovation (Goldberg 2012).

\subsubsection{Technology Acceptance Model: applications, extensions and SEM in healthcare}

Information systems surround us everywhere we go, at home, in the office. We interact with them, like them, dislike them, use them, complain, accept, reject. The framework of user acceptance of information technology is at heart of 
Technology Acceptance model (TAM). TAM is a robust and powerful predictive model (King and He 2006). It is used by researchers in technology, psychology, social sciences, healthcare and others (Dulcic et al. 2012; Choi and Totten 2012; Kukafka et al. 2003; Legris et al. 2003; Fensli et al. 2008).

\subsubsection{TAM and its derivatives}

In 1985, Fred Davis of MIT presented a dissertation entitled "A Technology Acceptance Model for Empirically Testing New End-User Information Systems: Theory and Results" (Davis 1985). His work was centered toward improving the understanding of user acceptance process for successful design and implementation of information systems and providing theoretical basis for a practical methodology of "user acceptance" through TAM, which could enable implementers and systems designers to evaluate proposed systems (Davis 1985). The paradigm for his research was a theoretical model of human behavior from psychology, grounded with empirical support. Davis then stabled measures for model's psychological variables and validated them through a field survey of 100 organizational users, and further tested the model through a laboratory user acceptance experiment of two business graphics systems (Davis 1985). The meaning of conceptual framework from Davis rests in the main point that the actual system use (the response) is the result of the stimulus of system features and capabilities that drives the 'organism' of users' motivation to use the system.

His proposed model sheds light on the behavioral part of the concept, with overall attitude of a potential user toward system use being a main determinant 
of the system's use. On the other hand, perceived usefulness and perceived use are outlined to be the main two variables influencing attitude toward using the system. Perceived usefulness is "the degree to which individual believes that using a particular system would enhance his or her job performance". Perceived ease of use is "the degree to which an individual believes that using a particular system would be free of physical and mental effort". He argues that system that is easier to use will result in increased job performance and greater usefulness for the user all else being equal. Davis also shows that perceived ease of use has a causal effect on the variable of perceived usefulness (Davis 1985; Davis and Venkatesh 1996). Attitude toward use is referred to as the degree of evaluative effect that an individual associates with using the target system in his/her job, while actual system use is the individual's direct usage of the given system (Davis 1985; Davis and Venkatesh 1996).

Described mathematically, TAM will look like this (Davis, 1985):

Perceived ease of use $(E O U)=\Sigma_{i=1, n} \beta_{i} X_{i}+\varepsilon$;

Perceived usefulness $($ USEF $)=\sum_{i=1, n} \beta_{i} X_{i}+\beta_{n+1}$ EOU $+\varepsilon$;

Attitude toward using $($ ATT $)=\beta_{1}$ EOU $+\beta_{2}$ USEF $+\varepsilon$;

Actual use of the system (USE) $=\beta_{1}$ ATT $+\varepsilon$,

where

$X_{i}$ is a design feature $I, i=1 \ldots n$; 
$\beta_{i}$ is a standardized partial regression coefficient;

$\varepsilon$ is a random regression term.

Later, in his 1993 journal article, Davis further tested TAM model expressed by the structural equations researching the use of electronic mail system and text editor. The questionnaire was distributed to 112 professional and managerial employees of a large North American corporation with the response rate of $93.3 \%$ (Davis 1993). According to the results of the study, system did not have a direct effect on use, but significant effect on attitude toward using. Perceived usefulness had a strong and significant effect on attitude, while ease of use had a smaller but also significant effect on attitude. Ease of use also had a strong and significant effect on perceived usefulness (Davis 1993). There could be response bias, and the author recommended further studies on that as well as including other variables that strongly correspond to a given system like system familiarity, experience, top management support, user involvement, task characteristics (complexity), design features etc. The article also has a strong point that while user friendliness and ease of use is important, and lots of researchers focus on user interfaces that increase usability, usefulness is even more important, should not be overlooked and no amount of ease of use can compensate the reality of the usefulness of the system (Davis 1993).

Davis also proposed several other variations of TAM emphasizing the importance of deeper understanding of the causal mechanisms that link system design attributes to user motivation. Observed related variables that causally 
intervene among existing variables have to be defined. He noted that a more detailed specification of the model could generate insights regarding user motivation and provide basis for more powerful diagnostic tools for proposed system design evaluation (Davis 1985).

In his 1999 article in International journal of medical informatics David Dixon combined Roger's innovation diffusion theory and Davis' TAM into information technology adoption model (ITAM) (Dixon 1999). While the link between perceived ease of use and perceived usefulness is intact, the rest of the model differs from the original (Dixon 1999).

Later, in 2000, Venkatesh and Davis proposed another TAM2 - extension of TAM, that differs from the original hypothesized TAM2 (Venkatesh and Davis 2000).

Other researchers proposed various frameworks for analysis adoption process. Thus, Frambach and Schillewaert integrated research on innovation adoption and technology acceptance and addressed the adoption decision in two levels: organizational and individual adopter (Frambach and Schillewaert 2002). Some of the perceived innovation characteristics included compatibility, complexity and relative advantage, while environmental influences were network externalities and competitive pressures. Social network for innovation adoption framework, according to authors, includes interconnectedness and network participation, while organizational facilitators include training, social persuasion and organizational support (Frambach and Schillewaert 2002). 


\subsubsection{Theory of Reasoned Action}

Perceived behavioral control and goal-oriented behavior has been studied by Ajzen and Fishbein, who proposed theory of reasoned action (TRA) (Ajzen and Madden 1986). The fundamental point of TRA is that the immediate precedent of any behavior is the intention to perform behavior in question. Stronger intention increases the likelihood of performance of the action, according to the theory (Ajzen and Madden 1986). Two conceptually independent determinants of intention are specified by TRA:

- attitude toward the behavior (the degree to which individual has favorable evaluation of behavior in mind or otherwise);

- subjective norm (perceived social pressure whether the behavior should be performed or not i.e. acted upon or not)

TRA also states that the behavior is a function of behavioral beliefs and normative beliefs, which are relevant to behavior (Ajzen and Madden 1986). Ajzen and Madden (1986) note, that TRA is insufficient whenever control over the behavioral goal is incomplete, since intention in the theory is the sole predictor of behavior. Other factors could interfere with behavior, whether internal (skills, abilities, planning, knowledge) or external (opportunity, time, dependence on others). The estimate of the extent to which the individual is capable of employing control over the behavior in question needs to be assessed in order to predict behaviors over which individual have only limited control (Ajzen and Madden 1986). Davis (1989) notes that TRA does not specify the beliefs that are 
functioning for a particular behavior; moreover, researchers should identify salient beliefs for people regarding behavior in question.

TAM was formulated as an adaptation and a backdrop of TRA for modeling theoretical relationships between variables dealing with cognitive and affective determinants of computer acceptance (Davis 1989). TAM does not include subjective norm as a determinant of behavioral intention. Instead, it notes that computer usage is determined by behavioral intention, which is jointly depends on person's attitude toward a system and perceived usefulness. In fact, another modified version of TAM presented by Davis, show behavioral intention as the result of attitude toward use and perceived usefulness. The meaning of the relationship of attitude toward use and behavioral intention to use is the philosophy that "people form intentions to perform behaviors toward which they have positive affect", while the connection between perceived usefulness and behavioral intention to use is based on the idea that people in the organization form intentions toward certain behavior that they believe would increase their job performance. Some studies show a significant influence of subjective norm on perceived usefulness and behavioral intention to use and have modified TAM to include subjective norm variable (Schepers and Wetzels 2007; Hung et al. 2012).

\subsubsection{Theory of planned behavior (TPB)}

In 1985, in a Journal of Experimental Social Psychology, researchers Icek Ajzen and Thomas Madden tested a theory of planned behavior and theory of reasoned action in two experiments involving college students' attendance and 
behavioral goal (of getting an "A"). According to their results, theory of planned behavior permitted more accurate prediction of goal attainment as well as prediction of intentions compared to the theory of reasoned action. Theory of planned behavior extends the theory of reasoned action by including the concept of behavioral control. The authors explain that the importance of control could be observed through the fact that the resources and opportunities available to individuals have to dictate to some extent the likelihood of behavioral achievement (Ajzen and Madden 1986). According to the TPB, a set of beliefs that deals with the presence or absence of requisite resources and opportunities could ultimately determine intention and action. In that view, the more opportunities and resources individual think they possess, the fewer obstacles and impediments they anticipate and the greater their perceived control over behavior should be (Ajzen and Madden 1986).

The researchers also concluded that the addition of perceived behavioral control greatly improved the model's predictive power, which showed that theory of reasoned action (that implies that perceived behavioral control can influence intention indirectly via attitude or subjective norm) does not adequately account for their data (Ajzen and Madden 1986). Holden and Karsh (2010) analyze some TAM studies and show extra variables used in TAM and related models.

\subsubsection{IT adoption in hospitals}

The review of the intention-based theories of IT adoption shows that adoption and use of technological innovations is ultimately defined by the user 
beliefs and attitudes towards information systems. Since 1985, TAM has been tested by researchers in many fields, and in healthcare industry there has been a need examine capabilities of TAM for healthcare IT, particularly in the era of a strong push for electronic health records and computerized decision-support system.

One recent study looked at the use of information technology in hospitals through the lens of TAM, particularly to examine HIS acceptance by Greek hospital personnel (Aggelidis and Chatzoglou 2009). Reliability and validity of the model was tested through correlation, explanatory and confirmation factor analysis. Causal model has been evaluated using structural equation modeling (SEM) technique.

The researchers display an interesting derivative of TAM, including social influence, training, facilitating conditions as well as anxiety and self-efficacy and computer attitude. The model is shown as a framework of three contexts: technological, implementation and individual with a number of hypotheses tested. The research method consisted of a preliminary questionnaire to 50 HIT users and validation from 3 experts in the field, followed by the main questionnaire to 341 HIS users from all main public hospitals in the East Macedonia and Thrace regions of Greece, which received $83 \%$ response rate (Aggelidis and Chatzoglou 2009). Composition of the sample was skewed towards administrative personnel $(72.8 \%)$. The main factor that was shown to positively affect behavioral intention was facilitating conditions (new computers, support during information system 
usage, financial rewards). Also, it appeared that facilitating conditions, perceived usefulness and self-efficacy reduce anxiety during system usage. Perceived usefulness and ease of use found to be the most important factors directly influencing behavioral intention, while facilitating conditions and training were leading in total effects influences (Aggelidis and Chatzoglou 2009).

In comparison, we can look at the study that focuses on the application of TAM in order to explain the intention to use clinical information systems, where the random sample consisted of 604 medical staff (534 physicians) in 14 hospitals in Greece (Melas et al. 2011). The researchers used confirmatory factor analysis (CFA), SEM and multi-group analysis of structural invariance (MASI).

The results of this study clearly show the direct effect of perceived ease of use on behavioral intention to use. Extending the model by including ICT feature demands factors (how sophisticated ICT must be before clinicians would be willing to use them) and ICT knowledge (how much knowledge clinicians believe to have about ICT) revealed a good fit to the data (Melas et al. 2011). No significant effects though were found of two external factors on behavioral intention to use. Positive relationships were found between PEoU and PU and between ATT and BI. The researchers indicated that the results of structural equation modeling confirm the predictive power of TAM and support positive relationships found in previous health care projects research. Greek studies may not be generalized to other populations. The comparison with studies from other countries could show some trends and reveal other results. 
Another study with the use of modified TAM in order to understand the factors affecting acceptance of information technology by healthcare professionals was performed by researchers in Taiwan (Chen and Hsiao 2012). The survey methodology was used toward physicians in the selected hospital with 202 questionnaires send out, yielding 124 completed ones, or $61.4 \%$ response rate (Chen and Hsiao 2012). The researchers used confirmatory factor analysis in AMOS 17.0 software to assess the reliability and validity of the model and the maximum likelihood method to estimate the parameters of the research model. SEM was used for the causal model estimation. According to the results, top management support had a significant impact on perceived usefulness, and project team competency and system quality had a significant impact on perceived use of hospital IT. Again, as previous research showed, the main variables of TAM, perceptions of usefulness and ease of use had a significant impact on acceptance of the systems.

Another study from Taiwan proposed a research model based on decomposed TBP in order to examine the factors influencing physicians' acceptance of the Medline system (a biomedical research literature database). The researchers believe that TAM was not appropriate for examining technology acceptance among physicians; therefore, modified TBP was used (Hung et al. 2012). The data from 224 physicians was analyzed using SEM approach with partial least squares (PLS) method (Hung et al. 2012). Their results indicate that the physicians' intention to use is significantly impacted by attitude, subjective 
norm and perceived behavior control, the factors that could be predicted by perceived usefulness, perceived ease of use, interpersonal influence, personal innovativeness in IT and self-efficacy.

There is another study from Taiwan that looks at the adoption by a particular group of medical personnel - nurses. Cheng (2012) looked at the role and relevance of interaction factors, flow - intrinsic motivator and extrinsic motivators - perceived usefulness and perceived ease of use in explaining intention to use the e-learning system by nurses. Two regional hospitals were used to gather the data. Usable questionnaire response rate was $68 \%$ - out of total 320 surveys distributed (Cheng 2012). The researcher constructs extended TAM while presenting three types of interaction factors: learner-system, instructor-learner and learner-learner. Confirmatory factor analysis (for the measurement model development) and structural model (for causal exploration among the constructs) was deployed and tested by SEM.

Lerner-system interaction as well as instructor-learner and learner-learner had significant effects on perceived usefulness, perceived ease of use and flow. Flow had significant effects on PU and PEoU. There is strong relationship between PEoU and PU. The influences of PU, flow and PEoU on intention to use was also significant. Flow was measured by three statements that are borderline ease of use and could be interpreted as enjoyment of the system use. The statements are the following (Cheng 2012):

- I am absorbed in what I am doing while using the e-learning system; 
- I find using the e-learning system to be enjoyable

- I am often unable to keep track of the passage time while using the elearning system.

An interesting study was done by Canadian researchers that tested a portion of TAM, particularly correlations of perceived usefulness of clinical and administrative applications (Paré and Sicotte 2001). IT sophistication was characterized in three dimensions (functional, technological and integration). They found that IT sophistication and perceived usefulness of clinical applications are moderately to highly correlated while no relationship was found between the level of sophistication and perceived usefulness of administrative applications in Quebec, Canada (Paré and Sicotte 2001).

Physicians' acceptance of telemedicine was done in Hong Kong at 41 out of 70 departments of 8 public acute-care tertiary hospitals (Chau and Hu 2002). The researchers tested TPB, TAM and integrated model of both to explain physicians' technology acceptance decisions and concluded that TAM was found more appropriate for examining technology acceptance (Chau and Hu 2002).

A recent study in France by Trevor Moores revisits TAM and modifies it for applications in adoption of a clinical management system for hospital workers (Moores 2012). Some interesting findings are the differences in significant impacts depending on the experience of the users. The research model has been tested using partial least squares (PLS) and Smart PLS (Moores 2012). Abbreviated constructs for the model are: ACC - accuracy [of information 
quality]; CNT - content; FMT - format; TIM - timeliness; CSP - computing support; EFF- self-efficacy; INFQ - information quality; ENBF - enabling factors; PU - perceived usefulness; PEOU -- perceived ease of use; USE - actual system use; ATT - attitude toward using; COM - compatibility.

A longitudinal study into primary care practitioners' views of and electronic medical records system (EMR) for maternity patients conducted in a large urban hospital in New Zealand reported the findings of the first phase that perceived usefulness and ease of use were important to medical personnel and other features of proposed system influenced the respondents' willingness to use the system (Handy et al. 2001). In the United Kingdom the researchers used TAM to investigate the acceptance of a computerized decision-support system in primary care (Van Schaik et al. 2004). The researchers outlined the need to consider the balance of perceived advantages (benefits) and disadvantages (costs) of a new system in modeling of technology acceptance (Van Schaik et al. 2004). Another very recent study in Hong Kong looks into factors affecting intentions to use the clinical imaging portal based on the TAM extended by inclusion of an external variable: computer self-efficacy (Chow et al. 2012a). A study by Taiwanese researchers employed updated TAM model to study healthcare information systems adoption by district nurses, head directors and other related personnel (Pai and Huang 2011). Questionnaire and SEM analysis was used. The model included information quality, service quality and system quality. Another study in Taiwan for mobile healthcare acceptance had different external variables: 
compatibility, MHS self-efficacy and technical support \& training (Wu et al. 2007). Their results were the following: compatibility, perceived usefulness and perceived ease of use significantly affected the behavioral intent (BI); MHS efficacy had strong indirect impact on $\mathrm{BI}$ through PU and PEoU; technical support and straining had strong impact on MHS efficacy and no significant effect on PU and PEoU (Wu et al. 2007).

A rare study in Germany of physician's acceptance of e-health in ambulatory care had an interesting SEM model with 6 external variables (Dünnebeil et al. 2012). The researchers indicated that the diversities of the public systems around the world should be integrated into TAM research to correctly explain its drivers. The results of their study show that the perceived importance of standardization and perceived importance of current IT utilization were the most significant drivers for accepting e-health (Dünnebeil et al. 2012).

\subsubsection{Other health technology assessment methods}

While the use of HDM is not widely observed in healthcare technology assessment, some researchers like Degoulet et al. have developed frameworks for managing complex health information system (Degoulet et al. 1995). The abovementioned researchers proposed evaluation criteria that include functional, technical, organizational, medical, cultural, ethical, economic and industrial components for development and integration of the health care professional multimedia workstation (Degoulet et al. 1995). They looked at the problems of evaluation (reference evaluation criteria), design (workstation reference 
architecture, implementation (engineering environment) and installation (enterprise infrastructure). The researchers looked at categories of criteria, main actors and questions. Table 7 below depicts such categories (Degoulet et al. 1995).

Table 7 Evaluation criteria for development and integration of the health care professional multimedia workstation based on Degoulet et al. (1995).

\begin{tabular}{|l|l|}
\hline $\begin{array}{l}\text { Categories of } \\
\text { criteria }\end{array}$ & Sub-criteria \\
\hline Functional & Coverage, adaptability \\
\hline Technical & $\begin{array}{l}\text { Architectural (extensibility, maintainability, modularity, } \\
\text { portability, openness, interoperability, reusability, degree } \\
\text { of integration) } \\
\text { Quality and performances (acceptability, ease of use, } \\
\text { time response, reliability, security) }\end{array}$ \\
\hline Organizational & $\begin{array}{l}\text { End-user's participation, support for group decisions, } \\
\text { changes in the relationship between actors }\end{array}$ \\
\hline Medical & Quality of care, medical efficacy, effectiveness \\
\hline $\begin{array}{l}\text { Cultural and } \\
\text { ethical }\end{array}$ & $\begin{array}{l}\text { educational and research value, auditability, } \\
\text { patients'/professionals' privacy }\end{array}$ \\
\hline Economic & Direct and indirect costs, return on investments \\
\hline $\begin{array}{l}\text { Industrial and } \\
\text { commercial }\end{array}$ & $\begin{array}{l}\text { Industrial commitment, investments, market identification, } \\
\text { market share }\end{array}$ \\
\hline
\end{tabular}

While the study gave examples of the criteria and subcriteria for evaluation, it didn't employ or suggest the methodology to quantify the criteria for the decision-makers. In my proposed study, HDM will address this gap.

Other researchers (Chiasson et al. 2007) made a great contribution to the field by outlining and comparing the fields of medical informatics (MI) and information systems (IS).

The study by Angst, Agarwal, Sambamurthy and Kelly was looking into the diffusion of electronic medical records in the population of hospitals in the United 
States (Angst et al. 2010). The data used came from an annual nationwide survey of care delivery organizations in the United States (HIMSS Analytics database) and contained information about 3, 989 hospitals over 1975-2005 time span. The researchers used heterogeneous diffusion model technique to test the research hypothesis, which estimates a model of the following form:

$h_{n}(t)=\exp \left[\propto X_{n}+\sum_{s \in S_{n(t)}}\left(\beta V_{n}+\gamma W_{s}+\delta Z_{n s}\right)\right]$ (Angst et al. 2010),

where $h_{n}(t)$ is the hazard of the event of interest for case $n$ at time $t$; $X_{n}$ is a covariate vector describing the intrinsic propensity of $n$ to experience the event (adopt);

$\propto$ is a corresponding vector of parameters, net of contagion influences; $S_{n(t)}$ is a set of prior adopters that influence $n$;

$V_{n}$ is a covariate vector describing susceptibility of $n$ to contagious influence from $S_{i}$

$\beta$ is the corresponding vector of parameters;

$W_{s}$ is a covariate vector of variables that reflect the infectiousness of $s$ in influencing $n$;

$\gamma$ is the corresponding vector of parameters;

$Z_{n s}$ is a covariate vector of proximity variables for $\mathrm{n}$ and $\mathrm{s}$ (pairwise-specific influence of $s$ on $n$ );

$\delta$ is the corresponding vector of parameters. 
The study stressed the importance of social relationships in firm behaviors, saying that investigations of innovation adoption that ignore intrapopulation linkages would likely be incomplete (Angst et al. 2010). The control variables HITC (sum of HIT applications implemented), PROFIT (hospital's profit vs. not-for-profit status), TEACH (type of hospital whether it is teaching/research or not) and IS BUDGET (percentage of budget devoted to information systems) showed up as significant, while HITINIT (a count of the number of HIT initiatives underway within each state at the time of the research), SIZE (size of a hospital) and AGE (age of a hospital) were not significant. Researchers also saw significant differences between Northeast and West regions of the United States.

Nurses acceptance of electronic health records prompted a model that included EHR usability, EHR usefulness functionalities (CPOE, eMAR, nursing flowsheet) influencing EHR acceptance (Carayon et al. 2011). A study on HIT adoption by people with physical disabilities due to neurological conditions used regression analysis on 330 data showed that a person's intention to use the system increases with growing PU and PEoU; there is a negative interaction between PU and disability, positive interaction between PEoU and disability (Liang et al. 2011).

\subsubsection{Patients' adoption of healthcare IT}

There are some studies done in trying to explain adoption of healthcare IT by patients. In one study, the purpose was to identify major factors that could affect patients' perceptions of a Home Telecare Management System (HTMS) 
and develop a theoretical framework of patience acceptance of HTMS (Rahimpour et al. 2008). A total of 10 focus groups interviews with patients suffering from congestive heart failure and/or chronic obstructive pulmonary disease were conducted in Sydney, Australia. Video demonstrations and HTMS prototype was shown in order to ask the participants questions about their perceptions. The results of the study showed that while the respondents perceived system as useful and convenient, they had concerns with the issues of cost, ease of use, clinical support, low self-efficacy and use anxiety (Rahimpour et al. 2008). The researchers proposed that the two constructs: HTMS selfefficacy and anxiety should be included in future models for HTMS acceptance.

Another study on user acceptance of health IT employed TAM and extended it to understand public's need towards health information and the role of antecedents of acceptance constructs when designing effective health information website (Kim and Chang 2006). Researchers from Korea constructed the model and then tested their hypothesis through sending out a nationally representative random sample survey, performing descriptive analysis and data analysis (SEM) (Kim and Chang 2006). Their results seemed different from typical TAM studies outcomes: the hypothesis of direct effect of PEoU on CS (customer satisfaction) was not accepted; while Post-CS hypothesis was accepted in all model and it could be concluded that there is a significant effect between user satisfaction and realization of it in practice (Kim and Chang 2006). 
Usage support and customization were significant in their impact on PU and PEoU (Kim and Chang 2006).

Patient's acceptance of provider delivered e-health [application that delivers encyclopedic health content with browse and search access] was studied by American researchers by sending out a questionnaire to subjects who had recently registered for access to e-health from a large healthcare provider in the Midwestern US (Wilson and Lankton 2004). SEM analysis was used to test hypothesis. Their study tested three theoretical models of IT acceptance, which performed well in predicting patients' behavioral intention, and could be used to predict e-health acceptance in advance of system development, researchers concluded (Wilson and Lankton 2004). Additional antecedents included info seeking, satisfaction and internet dependence (Wilson and Lankton 2004).

A study that aimed at exploring the factors influencing behavior and adoption of USB-based personal health records (PHR) among patients' in Taiwan included subjective norm, security \& privacy and computer self-efficacy (Jian et al. 2012). The study showed that the key factors affecting adoption were usage intention, perceived usefulness and subjective norm (Jian et al. 2012). Another study concerning patients in Singapore investigated Singaporean women's acceptance of using mobile phones to seek health information (Lim et al. 2011). Their construct included variables of self-efficacy, technological anxiety, mobile experience, internet experience and illness experience (Lim et al. 
2011). The results showed that PU and self-efficacy positively predicted the intention to use mobile phone to seek medical information (Lim et al. 2011).

One of important aspects and aims of health IT is to improve and sustain constant education of medical personnel as new technologies, research, medical techniques, drugs etc. become available. One study, done by researchers in Hong Kong looked into e-learning, particularly into development and evaluation of a virtual environment, the online 3Dworld Second Life (SL) for rapid sequence intubation (RSI) (Chow et al. 2012b). The findings show that the system was perceived as useful and some strategies need to be taken for boosting selfconfidence in using the system (the PEoU was neutral) (Chow et al. 2012b).

\subsubsection{Research in the United States}

Adoption of PDAs in a hospital in New York, USA, was studied by Arun Vishwanath, Linda Brodsky and Steve Shaha. The researchers mention failed health IT implementations due to adoption-related barriers like user resistance, lack of motivation to adopt, perceived lack of utility and perceived complexity of innovation (Vishwanath et al. 2009). They build extended and modified TAM with external variables like age, specialty, position in hospital, attitudes toward HIT, cluster ownership and split PU and PEoU in per-adoption and post-adoption variables, making it a closed system with behavioral intent depending from preadoption PU and PEoU and PDA use frequency factor depending on behavioral intent. 
Researchers noted that inclusion of user characteristics shed light on the individual-level factors influencing the pre-adoption beliefs about PDAs (Vishwanath et al. 2009). Diffusion theory-based constructs and the physician's general attitudes toward HIT and cluster ownership effected pre-adoption variables. Authors also came to conclusion that physicians who tend to have a positive attitude toward HIT tend to have more positive expectations from PDAs.

Authors of a recent study of physician's adoption of electronic detailing (edetailing - using digital technology by pharmaceutical companies to relay information to physicians) addressed the factors and proposed a model of adoption based on diffusion of innovation theories and TAM (Alkhateeb et al. 2009). Their model included innovation characteristics (perceived relative advantage, compatibility, complexity and trialability), communication (peer influence), social system (affiliation with academia and policy restrictions), and physicians' characteristics.

Similar to the previously described study by Chau and Hu, examining the acceptance of telemedicine technology in Hong Kong, a group of researchers looked at PDA acceptance in the United States, collected data from 222 physicians and tested it with LISREL software (Yi et al. 2006). The most significant determinant of physicians' technology acceptance was perceived usefulness, consistent with Chau and $\mathrm{Hu}$ (Yi et al 2006; Chau and $\mathrm{Hu}$ 2002). The researchers also found that a significant effect of perceived ease of use on 
perceived usefulness and that both subjective norm and perceived behavioral control had significant effects on behavioral intention (Yi et al. 2006).

\subsubsection{HIT Adoption in other countries}

Some countries had achieved major breakthroughs in healthcare IT adoption, particularly in electronic medical records (Bates et al. 2003). While the regulations and culture may vary and have impact on registry implementation and use, the research and experience should be observed and studied. Thus, in Australia, New Zealand and England were able to achieve high transition to electronic health records by providing financial support and offering incentives for electronically submitted claims, ensuring evidence-based decision support, developing a strategic framework and standardization (Bates et al. 2003).

Some researchers presented case studies of implementation of HIT in various countries and provided comparative analysis as well as experiences/lessons learned sections (Rosemann et al. 2010; Were 2010).

A team of researchers from Canada performed a lessons-learned study from HIT system implementation experience in seven countries and concluded that system's graphical user interface design quality, feature functionality, project management, procurement and previous experience of users affect outcomes of implementation (Ludwick and Doucette 2009). They also concluded that quality of care, patient safety and provider/patient relations were not affected by system implementation and socio-technical perspective complicate HIT deployment (Ludwick and Doucette 2009). 
Their model of insulating and risk factors is shown in Figure 3.

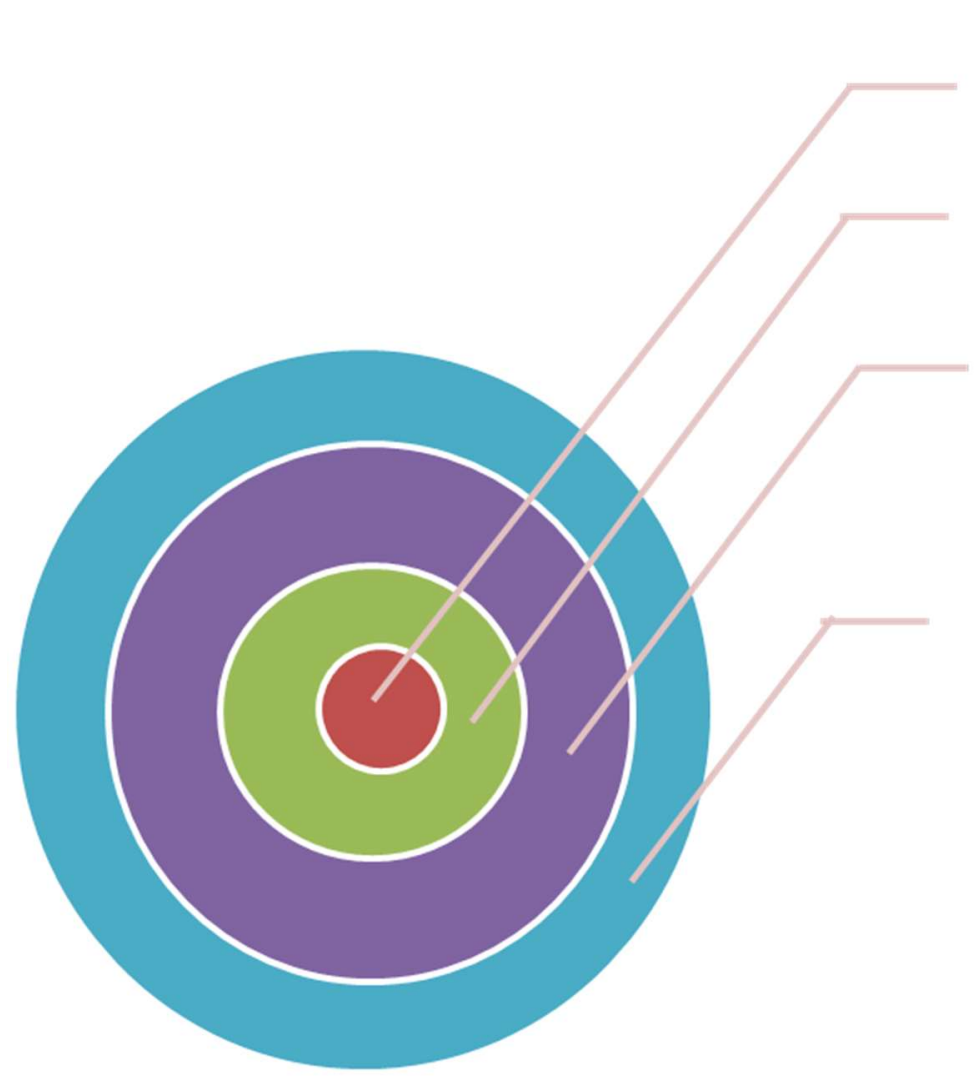

Goal

-Implementation Success

Fit Factor

-Socio-technical Interactions

Insulating Factors

-Project Management

- Leadership

- Training

-Standardization

Risk Factors

-Patient Safety

-Time

-Quality of Care

-Privacy

- Financial

-Efficiency

- Liability

-Previous Experience

-Staff Anxiety

-Patient/Provider Relationships

Figure 3 Model of insulating and risk factors (Ludwick and Doucette 2009).

\subsubsection{Quality Indicators in Healthcare Adoption and their Impacts}

While the attention of greater quality of care always persists, with research focus on how providers, patients and policies could affect factors that influence the quality of care, but despite high investments (over 1.7 trillion annually) and increased healthcare spending, U.S. ranks lower compared to other countries on 
several health measures (Jung 2006; Girosi et al. 2005; CMS 2014). Jung listed specific benefits of HIT in regard to quality of care:

- Medical error reduction (improved communication and access to information through information systems could have a great impact in this area)

- Adherence support (embedded decision support functions embedded can show the effect of HIT on adherence to guideline-based care and enhancing preventive healthcare delivery (Dexter et al. 2004; Overhage 1996; Jung 2006).

- Effective disease management (potential to improving the health outcomes of patients with specific diseases). Jung (2006) also explained that while efficiency is a complex concept, some efficiency savings have been reported by researchers as a result of HIT adoption as reduction in administrative time (Wong et al. 2003; Jung 2006) hospital stays from the increased patients' safety and care coordination etc. Positive effects on cost were documented as:

- Improved productivity

- Paper reduction

- Reduced transcription costs

- Drug utilization

- Improved laboratory tests 
Additional benefits, reported by several researchers (Bates et al. 1998; Agarwal 2002; Jung 2006) were:

- improved patient safety (from safety alerts and medication reminders)

- improved regulatory compliance (record keeping and reporting compliance with federal regulations including Health Insurance Portability and Accountability Act (HIPAA)).

Increased emphasis on preventive measures and early detection of diseases, primary care, intermittent healthcare services and continuity of care is prevalent in our ever-changing healthcare domain (Tsiknakis et al. 2002). Information and communication technologies are taking lead in this dynamic environment with the need for improved quality of healthcare services and costs control (Tsiknakis et al. 2002). Another important trend in the healthcare system is movement towards shared and integrated care, growth of home care through sophisticated telemedicine services (facilitated by intelligent sensors, hand-held technologies, monitoring devices, wireless technologies and Internet, which pushes the need for technology that supports quality and continuity of care (Tsiknakis et al. 2002; World Health Organization 2008). While the researchers enlisted a number of valuable benefits, they would need to be examined and the relationships and their significance would need to be studied further. The envisioned benefits are listed in Figure 4 and Table 8 below: 


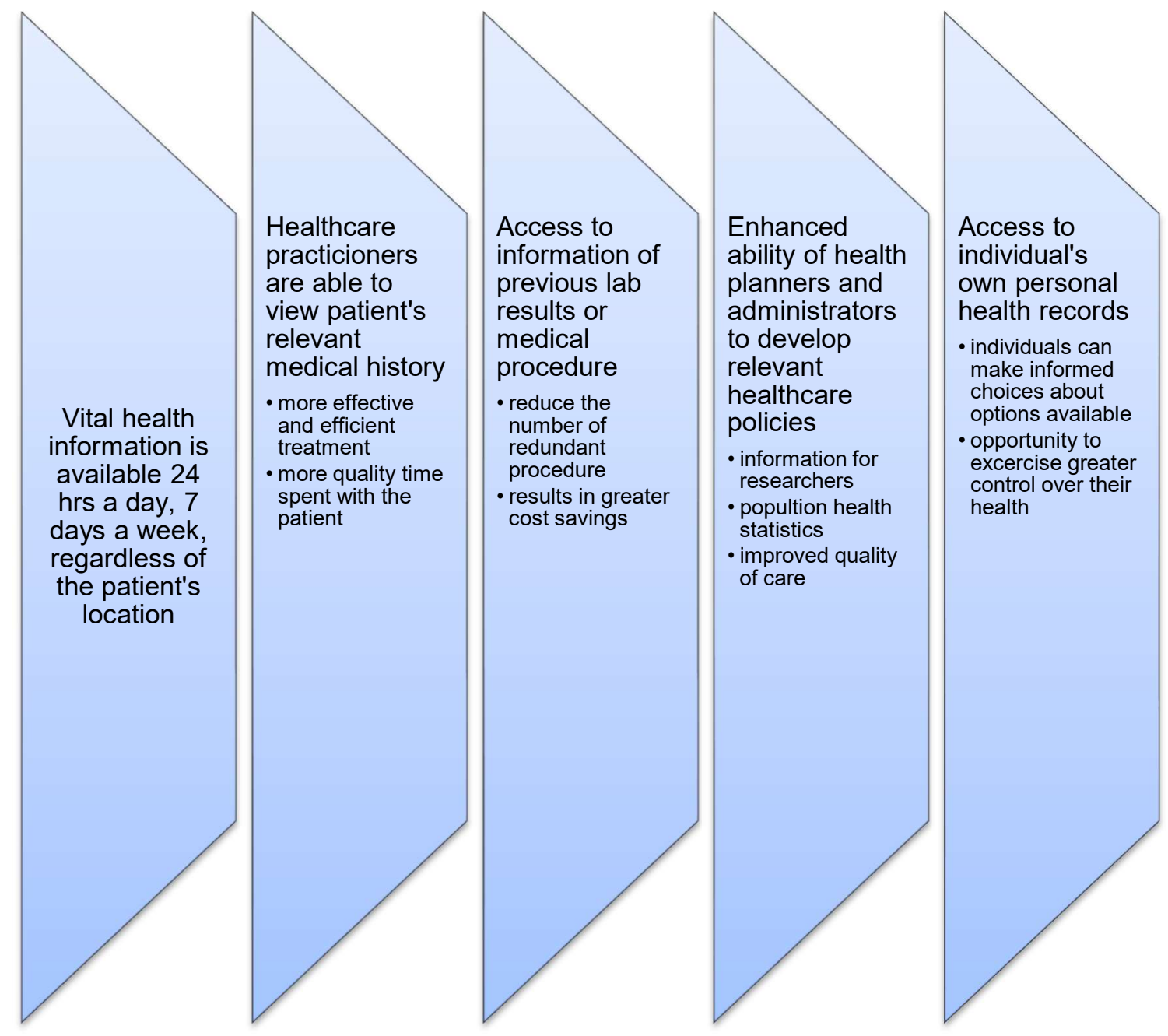

Figure 4 Envisioned health information technology benefits. 
Table 8 Potential benefits and their related features

\begin{tabular}{|c|c|}
\hline Potential benefit & $\begin{array}{l}\text { Related features of healthcare } \\
\text { information technology }\end{array}$ \\
\hline $\begin{array}{l}\text { Dissemination and distribution } \\
\text { of essential patient/client } \\
\text { information }\end{array}$ & $\begin{array}{l}\text { Open communication standards over } \\
\text { transparent platforms }\end{array}$ \\
\hline $\begin{array}{l}\text { Improved protection of personal } \\
\text { data }\end{array}$ & $\begin{array}{l}\text { Encryption and authentication } \\
\text { mechanisms for secure access to } \\
\text { sensitive personal information; auditing } \\
\text { capabilities for tracking purposes }\end{array}$ \\
\hline $\begin{array}{l}\text { Informed decision-making } \\
\text { resulting in improved quality of } \\
\text { care }\end{array}$ & $\begin{array}{l}\text { Semantic unification and multimedia } \\
\text { support for a more concise and complete } \\
\text { view of medical history }\end{array}$ \\
\hline $\begin{array}{l}\text { Prompt and appropriate } \\
\text { treatment }\end{array}$ & $\begin{array}{l}\text { Fast response times through transparent } \\
\text { networks and open interfaces }\end{array}$ \\
\hline $\begin{array}{l}\text { Risk reduction (access to a } \\
\text { wider patient/client knowledge } \\
\text { base) }\end{array}$ & $\begin{array}{l}\text { Appropriate usable human-computer } \\
\text { interfaces through awareness of } \\
\text { contextual factors }\end{array}$ \\
\hline $\begin{array}{l}\text { Facilitation of co-operation } \\
\text { between health professionals of } \\
\text { different levels of health social } \\
\text { care organization }\end{array}$ & $\begin{array}{l}\text { Role-based access mechanisms and } \\
\text { access privileges }\end{array}$ \\
\hline $\begin{array}{l}\text { Reduction in duplicate } \\
\text { recording/questioning of } \\
\text { relevant patient information }\end{array}$ & $\begin{array}{l}\text { A robust and scalable interface that } \\
\text { could extend from corporate/hospital to } \\
\text { regional and national level }\end{array}$ \\
\hline $\begin{array}{l}\text { More focused and appropriate } \\
\text { use of resources due to shared } \\
\text { information of assessment and } \\
\text { care plan }\end{array}$ & $\begin{array}{l}\text { Access to all diagnostic information } \\
\text { through adaptive user interfaces }\end{array}$ \\
\hline $\begin{array}{l}\text { Improved communication } \\
\text { between professionals }\end{array}$ & $\begin{array}{l}\text { Multimedia information is in the best } \\
\text { format by clinical information system for } \\
\text { communication without loss of quality }\end{array}$ \\
\hline $\begin{array}{l}\text { Security and guarantee of } \\
\text { continuity of care }\end{array}$ & $\begin{array}{l}\text { Permanent access and control of } \\
\text { interventions }\end{array}$ \\
\hline $\begin{array}{l}\text { Identification of a single patient } \\
\text { across multiple systems }\end{array}$ & $\begin{array}{l}\text { Mechanism for identifying a single client } \\
\text { record and associated data that may } \\
\text { have been stored on various source } \\
\text { systems }\end{array}$ \\
\hline $\begin{array}{l}\text { Consistent shared language } \\
\text { (between professionals) }\end{array}$ & $\begin{array}{l}\text { Mapping tool to display information in a } \\
\text { generic format to bridge the gap in } \\
\text { terminology and semantic differences }\end{array}$ \\
\hline
\end{tabular}


A systematic review by Goldzweig lists only a few studies of commercial health IT systems use with reported results and experiences of the impacts of EHR implementation (Goldzweig et al. 2009). In one of the studies described in their publication, authors concluded that health technology implementation (EpiCare at Kaiser Northwest) had no negative impact on quality of care: measures of quality like immunizations and cancer screening did not change (Goldzweig et al. 2009). In the second study of implementation of a commercial electronic health record in a rural family practice in New York, the authors report various financial impacts (average monthly revenue increase due to better billing practices); clinical practice satisfaction as well as the support of the core mission of providing care.

Agency for Healthcare Research Quality defined quality health care as "doing the right thing at the right time in the right way to the right person and having the best possible results" (Kazley and Ozcan 2008).

One important retrospective study in United States by Kazley and Ozcan looked at impacts of health information technology on quality performance in acute care hospitals (Kazley and Ozcan 2008). Retrospective cross-sectional format with linear regression is used in order to assess the relationship between HIT use in the hospital and quality performance (Kazley and Ozcan 2008). The authors concluded that there is a limited evidence of the relationship between technology use and quality. There are some interesting observations made by the authors towards measuring quality and they describe it as a multifaceted and 
complex construct, which may grow and change. Ten process indicators related to 3 clinical conditions: acute myocardial infarction, congestive heart failure and pneumonia are used to measure quality performance based on their validity (Kazley and Ozcan 2008). The authors noted that they didn't measure such elements of quality as patient satisfaction and long-term outcomes.

Leu et al. (2008) performed a qualitative study with in-depth semistructured interviews to describe how health IT functions within a clinical context. Six clinical domains were identified by the researchers: results management, intra-clinic communication, patient education and outreach, inter-clinic coordination, medical management and provider education and feedback. Created clinical process diagrams could provide clinicians, IT, and industry with a common structure of reference while discussing health IT systems through various time frames (Leu et al. 2008).

While it would be expected that health data would allow quality assessment and other impact assessment without expensive and timeconsuming processing of medical documentation, Roth et al. (2009) conclude that only about a third of indicators of the Quality Assessment Tools system would be readily available through health records with some concerns that only components of quality would be measured, perhaps to the detriment of other important measures of health care quality. The researchers provided a table of accessibility of quality indicators (clinical variables), which have been narrated in Table 9 below: 
Table 9 Accessibility of quality indicators

\begin{tabular}{|l|l|}
\hline $\begin{array}{l}\text { Accessible indicators (most to } \\
\text { least) }\end{array}$ & $\begin{array}{l}\text { Hard to access indicators } \\
\text { (most to least) }\end{array}$ \\
\hline Demographics & Disease-specific history \\
\hline Diagnosis & Care site \\
\hline Prescription & Physical exam \\
\hline Past medical history & Refusal \\
\hline Procedure date & Patient education \\
\hline Lab date & Social history \\
\hline Problem/chief complaint & Treatment \\
\hline Vital sign/weight/height & Diagnostic test result \\
\hline Allergy & Imaging result \\
\hline Lab result & Contraindication \\
\hline Medication history & Pathology \\
\hline Diagnostic test date & Family history \\
\hline Imaging date & EKG result \\
\hline Medications, current & X-ray result \\
\hline Vaccination & \\
\hline X-ray date & \\
\hline EKG date & \\
\hline
\end{tabular}

A group of researchers looked into problem of improving patient safety in ambulatory settings and throughout this qualitative study developed a tool kit of best practices and a collaborative to enhance medication-related practices and patient safety standards (Schauberger and Larson 2006). The list of best practices for the inpatient setting was the following, with \# 6, 10 and 3 being the top three process improvements on best practices:

1. Maintaining accurate and complete medication list

2. Ensuring medication allergy documentation

3. Standardizing prescription writing

4. Removing all IV potassium chloride from all locations

5. Emphasizing non-punitive error reporting 
6. Educating about look-alike, sound-alike drugs

7. Improving verbal orders

8. Ensuring safety and security of sample drugs

9. Following protocols for hazardous drug use

10. Partnering with patients

11. Notifying patients of laboratory results

Quality effect factors of information technology adoption were described by DesRoches et al. in the New England Journal of Medicine (DesRoches et al 2008). Those were:

- quality of clinical decisions;

- quality of communication with other providers;

- quality of communication with patients;

- prescription refills;

- timely access to medical records;

- avoiding medication errors;

- delivery of preventive care that meets guidelines;

- delivery of chronic-illness care that meets guidelines.

While the positive effect was shown in many cases, the significance of $p<0.001$ was reported only for the quality of clinical decisions; delivery of preventive care that meets guidelines and delivery of chronic-illness care that meets guidelines. 
Lanham, Leykum and McDaniel, who focused on social underpinning of HIT use or the "human element" of technology acceptance, implementation and use, also noted about research in the area of HIT impacts, particularly HIT influence of fundamental outcomes like cost and quality of healthcare delivery as well as reshaping organizational culture and clinical workflow (Lanham et al. 2012).

Goroll et al. (2008) also talked about impact on safety and impact on quality. Those types of technology impacts may be hard to assess, but are extremely important in growing the healthcare information management field and constantly improving it. Chaudhry et al. (2006) performed systematic review of the impact of HIT on quality, efficiency and cost. The researchers outlined the components of an HIT implementation (Chaudhry et al. 2006):

- Technological (for example, system applications)

- Organizational process change (workflow redesign)

- Human factors (user friendliness)

- Project management (archiving project milestones).

Chaudhry et al. (2006) also discussed what elements are behind the major effects of quality, efficiency and cost:

1. Effect on quality was predominantly in the role of increasing adherence (with decision support) to guideline- or protocol-based care. In addition to the mentioned variable, clinical monitoring based on large-scale screening and aggregation of data could show how health IT can support new ways 
of care delivery. Reduction of medication errors was also reported measure of the effect on quality.

2. Effects on efficiency

a. utilization of care (could be measured through the monetized estimates through the average cost of the examined service at the researched institution; could be analyzed through provided decision support (display of laboratory test costs, computerized reminders, display of previous test results, automated calculation of pretest probability for diagnostic tests) at the point of care;

b. provider time (Physician time could be examined in relation to computer use);

3. Effects on Costs (changes in utilization of services; cost data on aspects of system implementation or maintenance).

A summary table, indicating key points of the systematic review on impacts of HIT from Chaudhry et al. (2006) is displayed in Table 10 below: 
Table 10 Summary points of impact studies (Chaudhry et al. 2006)

\begin{tabular}{|l|}
\hline Main summary points of impact studies \\
\hline $\begin{array}{l}\text { Health information technology has been shown to improve quality through: } \\
\text { - increasing adherence to guidelines } \\
\text { - enhancing disease surveillance }\end{array}$ \\
\hline $\begin{array}{l}\text { Primary and secondary preventive care holds much evidence on quality } \\
\text { improvement. }\end{array}$ \\
\hline Decreased utilization of care is reported as the major efficiency benefit \\
\hline Effect on time utilization is mixed \\
\hline $\begin{array}{l}\text { Empirically measured data on the aspects of costs is limited and } \\
\text { inconclusive }\end{array}$ \\
\hline $\begin{array}{l}\text { Four benchmark research institutions supply most of high quality literature } \\
\text { on multifunctional HIT systems }\end{array}$ \\
\hline $\begin{array}{l}\text { Effect of multifunctional commercially developed systems is not well } \\
\text { documented }\end{array}$ \\
\hline Interoperability and consumer HIT impacts have little evidence \\
\hline Generalizability is a major limitation in the literature \\
\hline
\end{tabular}

One particular study by Yusof et al. 2008 examined previous models of information systems (IS) evaluation, particularly the IS Success Model and the IT-Organization Fit Model as well as introduced another HOT-fit model based on the system of human, organization and technology-fit factors.

Updated DeLone and McLean IS Success Model was developed in 2003, based on the original DeLone and McLean IS Success Model, introduced 20 years ago as a framework and model for measuring the complex-dependent variable in IS research (DeLone and McLean 2003).

The measures in the framework are included in the six system dimensions (Yusof et al. 2008; DeLone and McLean 2003):

- System Quality (the measures of the information processing system itself);

- Information Quality (the measures of IS output) 
- Service Quality (the measures of technical support or service)

- Information Use (recipient consumption of the output of IS)

- User Satisfaction (recipient response to the use of the output of IS)

- Net Benefits (IS impact overall).

While the model illustrates clear, grounded, well-observed and specific dimensions or impacts of IS success/effectiveness and their relationships, it does not include organizational factors, which have been included in HOT-fit model (Yusof et al. 2008). Before depicting HOT-fit model, there is another model that requires our attention in order to improve understanding of our research model.

IT-Organizational Fit Model was presented in 1991 by Scott Morton and includes both internal and external elements of fit. Model's internal fit is attained through combination and dynamic equilibrium of organizational components of business strategy, organizational structure, management processes, and roles and skills; while model's external fit is achieved due to formulation of organizational strategy grounded in environmental trends and market, industry and technology changes (Yusof et al. 2008). The enabler - IT is shown to affect the management process, also impacting organizational performance and strategy.

In 2008, Yusof et al. combined elements of both models to create humanorganization-technology fit (HOT-fit) framework and proposed it for applications in healthcare, while testing it with subjectivist, case study strategy approach, 
employing qualitative methods (Yusof et al. 2008). The researchers also presented examples of the evaluation measures of the proposed network.

Focused incentives, training and education in the right direction could speed up the process of adoption and use of more sophisticated information technology systems (Miller and Sim 2004).

The overview of the implementation barriers from the perspectives of various user groups (physicians, health care professionals, managers and patients) can show multiple facets of the issue, as well as finding commonalities in implementation factors, which was research by McGinn and others (McGinn et al. 2011). Below are the factors outlined to be common barriers for all user groups (McGinn et al. 2011):

- Design/technical concerns

- Privacy and security concerns

- Cost issues

- Lack of time and workload

- Motivation

- Productivity

- Perceived ease of use

- Interaction between a patient and a health professional

- Interoperability

- Familiarity and ability with the system 
Goldzweig et al. (2009) describe various instances in their research review where perceived barriers to adoption included resistance from physicians, system downtime, increase of physicians' time, inadequate computer skills, cost and inability to find a suitable system. While the surveys bring new interesting findings in barriers and adoption of health IT, there is a gap in research of significance of barriers and their impact on HIT adoption.

While examining a large body of literature, lots of researchers provided either qualitative or overall general empirical evaluation of technology acceptance model or other acceptance models (Chiasson et al. 2007; Dillon and Morris 1996; Im et al. 2008; Premkumar and Bhattacherjee 2008; Tsiknakis et al. 2002; Szajna 1996; Scott and Briggs 2009; Yang 2004; Yusof et al. 2008; or explored particular aspects of the HIT adoption (Burton-Jones and Hubona 2006; Cresswell and Sheikh 2012; Degoulet et al. 1995; Janczewski and Shi 2002; Jeng and Tzeng 2012; Folland 2006; Hagger et al. 2007; Karahanna and Straub 1999; Kim and Malhotra 2005; Lee and Xia 2011; Malhotra 1999; Martich and Cervenak 2007; McFarland and Hamilton 2006; Melone 1990; Shin 2010; Storey and Buchanan 2008; Viswanathan 2005), while actual applications of TAM and its derivatives (Jimoh et al. 2012; Mäenpää et al. 2009; Polančič et al. 2010; Ortega Egea and Román González 2011; Yu et al. 2009) are difficult to find in the United States.

Some literature sources reviewed had interesting frameworks of IT adoption in healthcare that differed greatly from TAM (Davidson and Heineke 
2007; Hatton et al. 2012) or were experiential in nature (André et al. 2008;

Ayatollahi et al. 2009). One study (Becker et al. 2011) showed radical modification of TAM into DART model (dynamic acceptance model for reevaluation of technologies) that refers to the dimensions of PU and PEoU. 


\subsection{Research Taxonomies and Gaps}

\subsubsection{Taxonomy of Technology Assessment methods}

Table 11 Taxonomy of Technology Assessment methods

\begin{tabular}{|c|c|}
\hline $\begin{array}{l}\text { Technology assessment } \\
\text { methods }\end{array}$ & Literature sources \\
\hline $\begin{array}{l}\text { System dynamics and } \\
\text { structural modeling }\end{array}$ & $\begin{array}{l}\text { Keller and Ledergerber 1998; Linston et al. 1979; } \\
\text { Hoyle 2012; Jackson 2001; Jackson 2003; Matsueda } \\
\text { 2012; Mueller 2008; Polančič et al. } 2010\end{array}$ \\
\hline Impact analysis & Coates 1974; Tran and Daim 2008 \\
\hline Scenario analysis & $\begin{array}{l}\text { Tran and Daim 2008; Brent 2012; Coates 1974; Miller } \\
\text { and Waller 2003; Liu et al. 2007; Ferng 2009; Pallottino } \\
\text { et al. 2005; Buytendijk et al. 2010; Bianco et al. 2014; } \\
\text { Zhang et al. } 2012\end{array}$ \\
\hline Delphi techniques & $\begin{array}{l}\text { Tran and Daim 2008; Meesapawong et al. 2014; } \\
\text { Förster and Von der Gracht } 2014\end{array}$ \\
\hline Risk assessment & $\begin{array}{l}\text { Hundy and Hamblin 1988; Wilhite 2004; Cyranoski } \\
\text { 2012; Linder and Sexton 2011; Ideker et al. } 2011\end{array}$ \\
\hline $\begin{array}{l}\text { Decision analysis, MCDA, } \\
\text { decision making and } \\
\text { technology acquisition } \\
\text { (AHP, HDM, MAU, fuzzy } \\
\text { AHP, fuzzy logic and expert } \\
\text { judgment) }\end{array}$ & $\begin{array}{l}\text { Merkhofer 1982; Cunningham and Lei 2008; Wang and } \\
\text { Hsieh 2014; Başoğlu et al. 2012; Auvinen et al. 2014; } \\
\text { van Blommestein and Daim 2013; Daim et al. 2013; } \\
\text { Daim et al. 2012a; Daim et al. 2012b; Abotah 2015; } \\
\text { Gerdsri and Kocaoglu 2009; Gerdsri and Kocaoglu } \\
\text { 2013; Riddell and Wallace 2011; Daim and Kocaoglu } \\
\text { 2008; Devlin and Sussex 2011; Thokala and Duenas } \\
2012\end{array}$ \\
\hline $\begin{array}{l}\text { Cost-benefit analysis } \\
\text { methods }\end{array}$ & $\begin{array}{l}\text { Gagnon 1991; Schwartz et al. 2011; Lee et al. 2010; } \\
\text { Jang and Lee } 2013\end{array}$ \\
\hline $\begin{array}{l}\text { Roadmapping and } \\
\text { technology development } \\
\text { envelope (TDE) }\end{array}$ & $\begin{array}{l}\text { Daim and Oliver 2008; Amer and Daim 2011; Carvalho } \\
\text { et al. 2013; Tran and Daim 2008; Lee et al. 2012; } \\
\text { Geum et al. 2011; Fleischer et al. 2005; Daim et al. } \\
\text { 2011, Gerdrsi } 2005\end{array}$ \\
\hline $\begin{array}{l}\text { Mathematical and synthesis } \\
\text { methods (conjoint analysis; } \\
\text { bibliography, patent } \\
\text { analysis, technology futures } \\
\text { analysis, social networking; } \\
\text { statistical analysis) }\end{array}$ & $\begin{array}{l}\text { Sharif and Sundararajan 1983; Grupp and Hohmeyer } \\
\text { 1986; Daim et al. 2006; Linstone et al. 1979; Shen et } \\
\text { al. 2010; Behkami and Daim 2012; Seneler et al. 2009; } \\
\text { Ahn et al. 2013; Liao et al. 2013; Lazoi et al. 2011; } \\
\text { Zhou et al. 2010; Karvonen et al. 2012; Chan et al. } \\
\text { 2010; Daim et al. } 2010\end{array}$ \\
\hline $\begin{array}{l}\text { Multi-perspective approach } \\
\text { using qualitative and } \\
\text { quantitative techniques }\end{array}$ & Thorn et al. 2011; Ahn et al. 2009 \\
\hline $\begin{array}{l}\text { Technology Acceptance and } \\
\text { Adoption and Diffusion }\end{array}$ & $\begin{array}{l}\text { Basoglu 2007; Kerimoglu et al. 2008; Tanoglu et al. } \\
2010\end{array}$ \\
\hline
\end{tabular}




\subsubsection{Taxonomy of Health Technology Assessment methods}

Table 12 Taxonomy of Health Technology Assessment Methods

\section{Health Technology \\ Assessment methods \\ System dynamics and structural modeling Decision-making}

Scenario analysis
Heuristics
Decision trees
Bayesian Belief Networks
Data mining and machine
learning
Cost-benefit analysis
methods
Roadmapping and
technology development
envelope (TDE)
Mathematical and
synthesis methods

Multi-perspective approach using qualitative and quantitative techniques Technology Acceptance and Adoption and Diffusion

\section{Literature sources}

Linston et al. 1979; Hastie et al. 2009

Pauker and Kassirer 1987; Schwartz et al. 1973; Pauker 1976; Howard 1966; Howard 2006; Raiffa 1997; Pearl 1988; Cooper 1986; Matheny and Ohno-Machado 2007; Duda et al. 2001; Brown 2008; Hastie et al. 2009; Bruce and Fries 2005; Swets 1979; Metz 1978; Ledley and Lusted 1959; Greenes 2007

van Genugten et al. 2003; Bierbooms et al. 2011; Banta et al. 1987

Davidson and Heineke 2007; Hatton et al. 2012

Bohanec 2000; Schwartz et al. 1973; Pauker 1976;

Greenes 2007; Duda et al. 2001

Pearl 1988; Cooper 1986; Matheny and Ohno-Machado 2007

Greenes 2007; Bruce and Fries 2005; Strome 2013

Metz 1978; Swets 1979; Bruce and Fries 2005

Geum et al. 2011

Greenes 2007; Ledley and Lusted 1959; Raiffa 1997; Howard 1966; Schwartz et al. 1973; Pauker 1976; Bohanec 2000; Burton-Jones and Hubona 2006; Cresswell and Sheikh 2012; Degoulet et al. 1995; Janczewski and Shi 2002; Jeng and Tzeng 2012; Folland 2006; Hagger et al. 2007; Karahanna and Straub 1999; Kim and Malhotra 2005; Lee and Xia 2011; Malhotra 1999; Martich and Cervenak 2007; McFarland and Hamilton 2006; Melone 1990; Shin 2010; Storey and Buchanan 2008; Viswanathan 2005

Banta and Bos 1991; Goroll et al. 2008

Chiasson et al. 2007; Dillon and Morris 1996; Im et al. 2008; Premkumar and Bhattacherjee 2008; Tsiknakis et al. 2002; Szajna 1996; Scott and Briggs 2009; Yang 2004; Yusof et al. 2008; Jimoh et al. 2012; Mäenpää et al. 2009; Polančič et al. 2010; Ortega Egea and Román González 2011; Yu et al. 2009 


\subsubsection{Taxonomy of HIT evaluation factors}

Table 13 Taxonomy of HIT evaluation factors - Financial

\begin{tabular}{|c|c|}
\hline Financial & Literature sources \\
\hline Start-up costs & $\begin{array}{l}\text { Schoen et al. 2006; Menachemi and Brooks 2006; } \\
\text { Boonstra and Broekhuis 2010; Cresswell and Sheikh } \\
\text { 2012; Zaroukian 2006; Palacio et al. 2009; Simon et } \\
\text { al. 2007; Fonkych and Taylor 2005; McGinn et al. } \\
\text { 2011; Valdes 2004 }\end{array}$ \\
\hline Ongoing costs & $\begin{array}{l}\text { Witter 2009; Martich ad Cervenak 2007; Boonstra and } \\
\text { Broekhuis 2010; Ash and Bates 2005; DePhilips } \\
\text { 2007; Police et al. } 2011\end{array}$ \\
\hline $\begin{array}{l}\text { Financial uncertainties (lack } \\
\text { of tangible benefits; lack of } \\
\text { financial return; } \\
\text { reimbursement) }\end{array}$ & $\begin{array}{l}\text { Chaudhry et al. 2006; Menachemi et al. 2008; } \\
\text { Goldzweig et al. 2009; Blumenthal } 2009\end{array}$ \\
\hline $\begin{array}{l}\text { Lack of financial resources } \\
\text { (in some sources referred to } \\
\text { as lack of capital, lack of } \\
\text { funding etc.) }\end{array}$ & $\begin{array}{l}\text { Shields et al. 2007; Robert Wood Johnson Foundation } \\
\text { 2010; Simon et al. 2008; Boonstra and Broekhuis } \\
\text { 2010; Bowens et al. 2010; Ash and Bates 2005; Shen } \\
\text { and Ginn 2012; Palacio et al. 2009; Goroll et al. 2008; } \\
\text { Lorenzi et al. 2009; Simon et al. 2007; Fonkych and } \\
\text { Taylor } 2005\end{array}$ \\
\hline
\end{tabular}

Table 14 Taxonomy of HIT evaluation factors - Technical

\begin{tabular}{|c|c|}
\hline Technical & Literature sources \\
\hline $\begin{array}{l}\text { Information quality } \\
\text { (accuracy, content, } \\
\text { format, timeliness) }\end{array}$ & $\begin{array}{l}\text { Moores 2012; Wu et al. 2007; Chen and Hsiao 2012; Liang } \\
\text { et al 2011; Cresswell and Sheikh 2012; Bodenheimer and } \\
\text { Grumbach 2003; Kim and Chang 2006 }\end{array}$ \\
\hline $\begin{array}{l}\text { Intensity of IT } \\
\text { utilization (data } \\
\text { security, } \\
\text { documentation, } \\
\text { technical support, } \\
\text { complexity, } \\
\text { customization, } \\
\text { reliability, } \\
\text { interconnectivity, } \\
\text { interoperability, } \\
\text { hardware issues) }\end{array}$ & $\begin{array}{l}\text { Dünnebeil et al. 2012; Chen et al. 2010; Lorence and } \\
\text { Churchill 2005; Zhang and Liu 2010; Tsiknakis et al. 2002; } \\
\text { Vedvik et al. 2009; Bates et al. 2003; Robert Wood Johnson } \\
\text { Foundation 2010; Miller and Sim 2004; Angst et al. 2010; } \\
\text { Handy et al. 2001; Greenhalgh et al. 2009; Chen and Hsiao } \\
\text { 2012; Menachemi and Brooks 2006; Boonstra and } \\
\text { Broekhuis 2010; Rosemann et al. 2010; Tyler 2001; } \\
\text { Rahimpour et al. 2008; Rind and Safran 1993; Bowens et al. } \\
\text { 2010; Valdes et al. 2004; Jian et al. 2012; Blumenthal 2009; } \\
\text { Yoon-Flannery et al. 2008; Ludwick and Doucette 2009; } \\
\text { Ortega Egea and Román González 2011; Palacio et al. } \\
\text { 2009; Goroll et al. 2008; Simon et al. 2007; Police et al. } \\
\text { 2011; Glaser et al. 2008 }\end{array}$ \\
\hline
\end{tabular}


Table 15 Taxonomy of HIT evaluation factors - Social/Organizational - Part 1

$\begin{array}{ll}\text { Social/organizational } & \text { Literature sources } \\ \text { Top management support } & \text { Chen and Hsiao 2012; Legris et al. 2003; Morton } \\ & \text { and Wiedenbeck 2009; André et al. 2008; Yusof et } \\ & \text { al. 2008; Kim and Chang } 2006 \\ \text { Project/team competency } & \text { Chen and Hsiao 2012; Yarbrough and Smith 2007; } \\ & \text { Chow et al. 2012b; Carayon et al. 2011; Zaroukian } \\ & 2006 \\ \text { Process orientation } & \text { Dünnebeil et al. 2012; Chiasson et al. 2007 } \\ \text { Standardization } & \text { Lanhan et al. 2012; Holden and Karsh 2010; Kumar } \\ & \text { and Aldrich 2010; Greenhalgh et al. 2009; Tsiknakis } \\ & \text { et al. 2002; Boonstra and Broekhuis 2010; Tyler } \\ & \text { 2001; Cresswell and Sheikh 2012; Leu et al. 2008; } \\ & \text { Helms and Williams 2011; Lapinsky et al. 2008; } \\ & \text { Ludwick and Doucette 2009; Zaroukian 2006; } \\ & \text { Lorenzi et al. 2009; Kazley and Ozcan 2008; } \\ & \text { Randeree 2007; Matysiewicz and Smyczek 2009; } \\ & \text { Wagner and Weibel 2005; Glaser et al. 2008 } \\ & \text { Greenhalgh et al. 2009; Janczewski and Shi 2002 } \\ \text { Angst et al. 2010; Morton and Wiedenbeck 2009; } & \begin{array}{l}\text { Piliouras et al. 2011; Boonstra and Broekhuis 2010; } \\ \text { Staff reallocation/employment }\end{array} \\ \text { Security/confidentiality/privacy } & \text { Rosemann et al. 2010; Tyler 2001; Rind and Safran } \\ & \text { 1993; Alper and Olson 2010; Bowens et al. 2010; } \\ & \text { Ash and Bates 2005; Dünnebeil et al. 2012 }\end{array}$


Table 16 Taxonomy of HIT evaluation factors - Social/Organizational - Part 2

\section{Social/organizational \\ (continued) \\ Incentives \\ Policy drawbacks and supports}

Transience of vendors Workflow redesign

\section{Literature sources}

Kumar and Aldrich 2010; Schoen et al. 2006; Goldzweig et al. 2009; Bates et al. 2003; Beckett et al. 2011;

Greenhalgh et al. 2009; Boonstra and Broekhuis 2010;

Rosemann et al. 2010; Ford et al. 2006; Cresswell and Sheikh 2012; Ash and Bates 2005

Witter 2009; Miller and Sim 2004; Schoen et al. 2006;

Chumbler et al. 2011; Goroll et al. 2008; Simon et al. 2008; Chen and Hsiao 2012; Vishwanath et al. 2009; André et al. 2008

Bates et al. 2003; Ford et al. 2006; Randeree 2007 Miller and Sim 2004; Dixon et al 2010; Menachemi and Brooks 2006; Boonstra and Broekhuis 2010; Chaudhry et al. 2006; Bowens et al. 2010; Furukawa 2011; Zandieh et al. 2008; Zaroukian 2006; Goroll et al. 2008; Lorenzi et al. 2009 
Table 17 Taxonomy of HIT evaluation factors - Personal

\begin{tabular}{|c|c|}
\hline Personal & Literature sources \\
\hline $\begin{array}{l}\text { Age, specialty, } \\
\text { position, } \\
\text { familiarity }\end{array}$ & $\begin{array}{l}\text { Vishwanath et al. 2009; Morton and Wiedenbeck 2010; Miller and Sim } \\
\text { 2004; Wu et al. 2007; Bergman-Evans et al. 2008; Jeng and Tzeng } \\
\text { 2012; Angst et al. 2010; Handy et al. 2001; Chen and Hsiao 2012; } \\
\text { Rosemann et al. 2010; Rahimpour et al. 2008; Ortega Egea and } \\
\text { Román González 2011; Pai and Huang 2011; Im et al. 2008; Police et } \\
\text { al. } 2011\end{array}$ \\
\hline Motivation & $\begin{array}{l}\text { Beckett et al. 2011; Dixon 1999; Wu et al. 2007; Yu et al. 2009; } \\
\text { Greenhalgh et al. 2009; Yarbrough and Smith 2007; Piliouras et al. } \\
\text { 2011; Frambach and Schillewaert 2002; Cresswell and Sheikh } 2012\end{array}$ \\
\hline Productivity & $\begin{array}{l}\text { Morton and Wiedenbeck 2009; DeLia et al. 2004; Bowens et al. 2010; } \\
\text { Yoon-Flannery et al. } 2008\end{array}$ \\
\hline $\begin{array}{l}\text { Personal } \\
\text { innovativeness }\end{array}$ & $\begin{array}{l}\text { Jeng and Tzeng 2012; Frambach and Schillewaert 2002; Vishwanath } \\
\text { et al. 2009; Moores 2012; Yi et al. 2006; Hung et al. } 2012\end{array}$ \\
\hline Self-efficacy & $\begin{array}{l}\text { Chow et al. 2012a; McFarland and Hamilton 2006; Chau and Hu } \\
\text { 2002; Dixon 1999; Wu et al. 2007; Legris et al. 2003; Yu and Gagnon } \\
\text { 2009; Chen and Hsiao 2012; Rahimpour et al. 2008; Cresswell and } \\
\text { Sheikh 2012; Wu et al. 2009; Kukafka et al. } 2003\end{array}$ \\
\hline Anxiety & $\begin{array}{l}\text { Ludwick and Doucette 2009; Wu et al. 2007; Yarbrough and Smith } \\
\text { 2007; Cheng 2012; Kukafka et al. 2003; Aggelidis and Chatzoglou } \\
\text { 2009; Storey and Buchanan } 2008\end{array}$ \\
\hline
\end{tabular}

Table 18 Taxonomy of HIT evaluation factors - Interpersonal

Interpersonal Literature sources

Doctor-doctor Wu et al. 2007; Yu and Gagnon 2009; Yarbrough and Smith 2007; Doctor-nurse Chen and Hsiao 2012; Frambach ad Schillewaert 2002; Cheng Doctor-patient 2012; Yang 2004; Dünnebeil et al. 2012; Liu and Ma 2005; Yusof et al. 2008; Chiasson et al. 2007 


\subsubsection{Research gaps framework}

After examining a large body of literature on health technology

assessment, acceptance and evaluation (Table 11, Table 12, Table 13, Table 14,

\section{Table 15,}

Table 16, Table 17, Table 18 and Table 19), major research gaps shown in

Figure 5 are the following:

1. A comprehensive hierarchical model that looks at the technology adoption potential of the wearable medical devices in departmental hospital settings has not been successfully introduced.

2. Multi-perspective approach utilizing financial, technical, organizational, patient and interpersonal criteria in one model for the purposes of assessing the technology adoption potential in healthcare has not been introduced. 
Table 19 Bibliographical review of types of studies performed in Health Technology Assessment and Adoption

\begin{tabular}{|c|c|}
\hline Type of study & Research works \\
\hline $\begin{array}{l}\text { Qualitative or empirical } \\
\text { evaluation of Technology } \\
\text { Acceptance Model or other } \\
\text { acceptance models }\end{array}$ & $\begin{array}{l}\text { Chiasson et al. 2007; Dillon and Morris 1996; Im et } \\
\text { al. 2008; Premkumar and Bhattacherjee 2008; } \\
\text { Tsiknakis et al. 2002; Szajna 1996; Scott and Briggs } \\
\text { 2009; Yang 2004; Yusof et al. } 2008\end{array}$ \\
\hline $\begin{array}{l}\text { Exploration of particular } \\
\text { aspects of the HIT adoption }\end{array}$ & $\begin{array}{l}\text { Burton-Jones and Hubona 2006; Cresswell and } \\
\text { Sheikh 2012; Degoulet et al. 1995; Janczewski and } \\
\text { Shi 2002; Jeng and Tzeng 2012; Folland 2006; } \\
\text { Hagger et al. 2007; Karahanna and Straub 1999; } \\
\text { Kim and Malhotra 2005; Lee and Xia 2011; Malhotra } \\
\text { 1999; Martich and Cervenak 2007; McFarland and } \\
\text { Hamilton 2006; Melone 1990; Shin 2010; Storey and } \\
\text { Buchanan 2008; Viswanathan } 2005\end{array}$ \\
\hline $\begin{array}{l}\text { Applications of TAM and its } \\
\text { derivatives in other countries }\end{array}$ & $\begin{array}{l}\text { Jimoh et al. 2012; Mäenpää et al. 2009; Polančič et } \\
\text { al. 2010; Ortega Egea and Román González 2011; } \\
\text { Yu et al. } 2009\end{array}$ \\
\hline $\begin{array}{l}\text { Frameworks of IT adoption in } \\
\text { healthcare (stage process } \\
\text { and heuristics) }\end{array}$ & Davidson and Heineke 2007; Hatton et al. 2012 \\
\hline $\begin{array}{l}\text { Frameworks of IT adoption } \\
\text { experimental in nature }\end{array}$ & $\begin{array}{l}\text { André et al. 2008; Ayatollahi et al. 2009; Becker et } \\
\text { al. } 2011\end{array}$ \\
\hline $\begin{array}{l}\text { Decision-making in } \\
\text { healthcare }\end{array}$ & $\begin{array}{l}\text { Pauker and Kassirer 1987; Schwartz et al. 1973; } \\
\text { Pauker 1976; Howard 1966; Raiffa 1997; Pearl 1988; } \\
\text { Cooper 1986; Matheny and Ohno-Machado 2007; } \\
\text { Duda et al. 2001; Brown 2008; Hastie et al. 2009; } \\
\text { Bruce and Fries 2005; Swets 1979; Metz 1978; } \\
\text { Ledley and Lusted 1959; Greenes } 2007\end{array}$ \\
\hline $\begin{array}{l}\text { Other applications of } \\
\text { hierarchical decision models }\end{array}$ & $\begin{array}{l}\text { Wang et al. 2013; Wang et al. 2010; Cleland and } \\
\text { Kocaoglu 1981; Sheikh et at. 2014; Sheikh 2013; } \\
\text { Chan et al. 2013; Iskin and Daim 2014; Chen et al. } \\
\text { 2009; Gerdsri and Kocaoglu 2008; Gerdsri and } \\
\text { Kocaoglu 2009; Phan and Kocaoglu } 2014\end{array}$ \\
\hline
\end{tabular}




\begin{tabular}{|c|c|c|}
\hline Research Gaps & Research Goals & Research Questions \\
\hline \multirow{2}{*}{$\begin{array}{l}\text { A comprehensive } \\
\text { hierarchical model that } \\
\text { looks at the technology } \\
\text { adoption potential of } \\
\text { the wearable medical } \\
\text { devices in } \\
\text { departmental hospital } \\
\text { settings has not been } \\
\text { successfully } \\
\text { introduced. }\end{array}$} & \multirow{2}{*}{\begin{tabular}{|l|} 
1. Define a \\
research \\
framework for \\
the technology \\
adoption \\
potential of the \\
wearable \\
medical devices \\
in departmental \\
hospital \\
settings.
\end{tabular}} & $\begin{array}{l}\text { What are the perspectives } \\
\text { and criteria for assessing } \\
\text { technology adoption potential } \\
\text { of the wearable sensor } \\
\text { products for pervasive care in } \\
\text { neurosurgery and } \\
\text { orthopedics? }\end{array}$ \\
\hline & & $\begin{array}{l}\text { What are the wearable sensor } \\
\text { products that have potential } \\
\text { for adoption for pervasive } \\
\text { care in neurosurgery and } \\
\text { orthopedics? }\end{array}$ \\
\hline \multirow{3}{*}{$\begin{array}{l}\text { Multi-perspective } \\
\text { approach utilizing } \\
\text { financial, technical, } \\
\text { organizational, patient } \\
\text { and interpersonal } \\
\text { perspectives in one } \\
\text { model for the purposes } \\
\text { of assessing the } \\
\text { technology adoption } \\
\text { potential in healthcare } \\
\text { has not been } \\
\text { introduced. }\end{array}$} & \multirow{3}{*}{$\begin{array}{l}\text { 2. Assess the } \\
\text { importance of } \\
\text { perspectives } \\
\text { and criteria and } \\
\text { the lower level } \\
\text { of HDM through } \\
\text { expert judgment } \\
\text { quantification. }\end{array}$} & $\begin{array}{l}\text { Which health technology } \\
\text { assessment perspectives } \\
\text { have the highest importance } \\
\text { from physician's perspective } \\
\text { according to the experts? }\end{array}$ \\
\hline & & $\begin{array}{l}\text { Which wearable sensor } \\
\text { product has the highest } \\
\text { adoption potential? }\end{array}$ \\
\hline & & $\begin{array}{l}\text { How does change in values } \\
\text { of perspectives impact the } \\
\text { changes of rankings of } \\
\text { wearable sensor products? }\end{array}$ \\
\hline
\end{tabular}

Figure 5 Framework of research gaps, goals and questions 


\section{CHAPTER 3. RESEARCH METHODOLOGY}

\subsection{Validation of Hierarchical Decision Modeling through Applications}

Hierarchical Decision Modeling is the methodology that is applicable when managers or researchers are faced with multilevel decision possibilities under conflicting objectives and criteria and complex problems. Hierarchical decision process, has been pioneered by Kocaoglu and Saaty, has been implemented in various industries for the purposes of evaluation of information technology or other objectives, where the problem could be viewed as a network of relationships. In HDM, each level of the hierarchy could consist of conflicting decision elements or multidimensional criteria that are relevant to the problem at hand (Kocaoglu 1983).

The figure similar to an example of a decision hierarchy from Kocaoglu's research is presented below (Figure 6): 


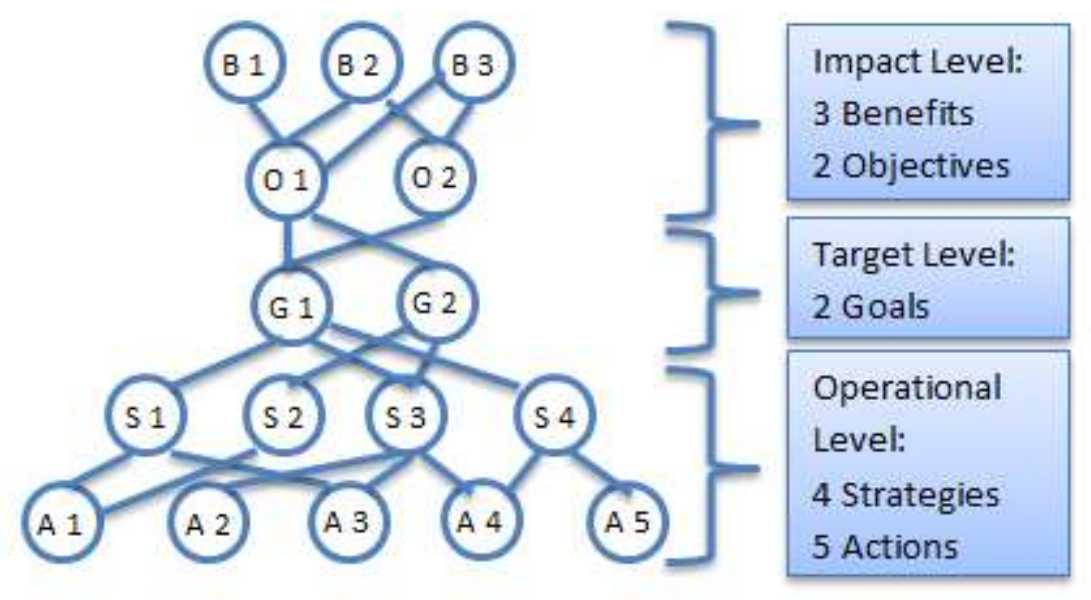

Figure 6 Example of a decision hierarchy.

Another example of generalized HDM model proposed by Kocaoglu and Cleland is MOGSA (Figure 7) (Cleland and Kocaoglu 1981). MOGSA consists of 5 hierarchical levels: Mission, Objectives, Goals, Strategies and Actions. Mission level usually answers the questions what business are we in or what business we want to be in. Objectives level represents the list of achievements we should have in order to satisfy our mission. Goals level reflects on the targets to reach in order to fulfil the objectives. Strategies, being a level under the Goals level, depict the pathways that should be followed in order to meet our goals. Actions level should outline the projects that are needed in order to develop our strategies. 


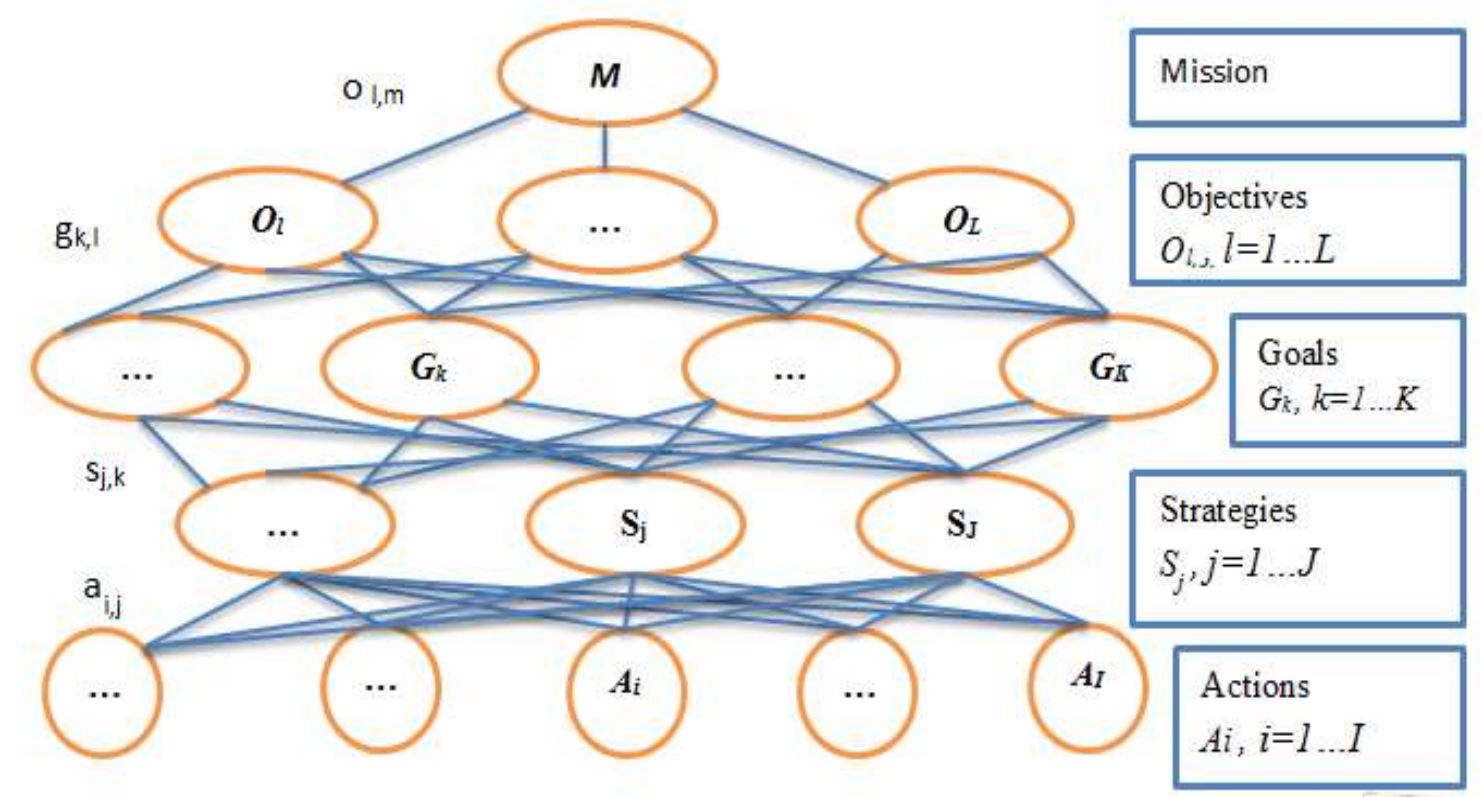

Figure 7 MOGSA research framework (Cleland and Kocaoglu 1981).

One of the most recent research papers using MOGSA was published in 2013 for selecting projects for non-profit organization (Wang et al. 2013).

Objectives in the model (for example: the advancement of education, the relief of poverty) were followed by organizational goals, other criteria and later quantified using pairwise comparison method.

Nasir Sheikh used HDM in assessment of solar photovoltaic technologies with multiple perspectives and hierarchical decision modeling (Sheikh et al. 2014). He looked at the problem through the lens of multiple perspectives, that he named STEEP: Social, Technological (or Technical), Economic, Environmental and Political. His research is another example that validates the use of HDM model for judgment quantification of decision makers to represent their world view (Sheikh et al. 2014). 
Another interesting research in hierarchical decision modeling was done by researchers Chan, Daim and Kocaoglu on developing a strategic policy choice framework for technological innovation (Chan et al. 2013). The model has four levels and the alternatives level is resource level. The model is depicted in Figure 8 below:

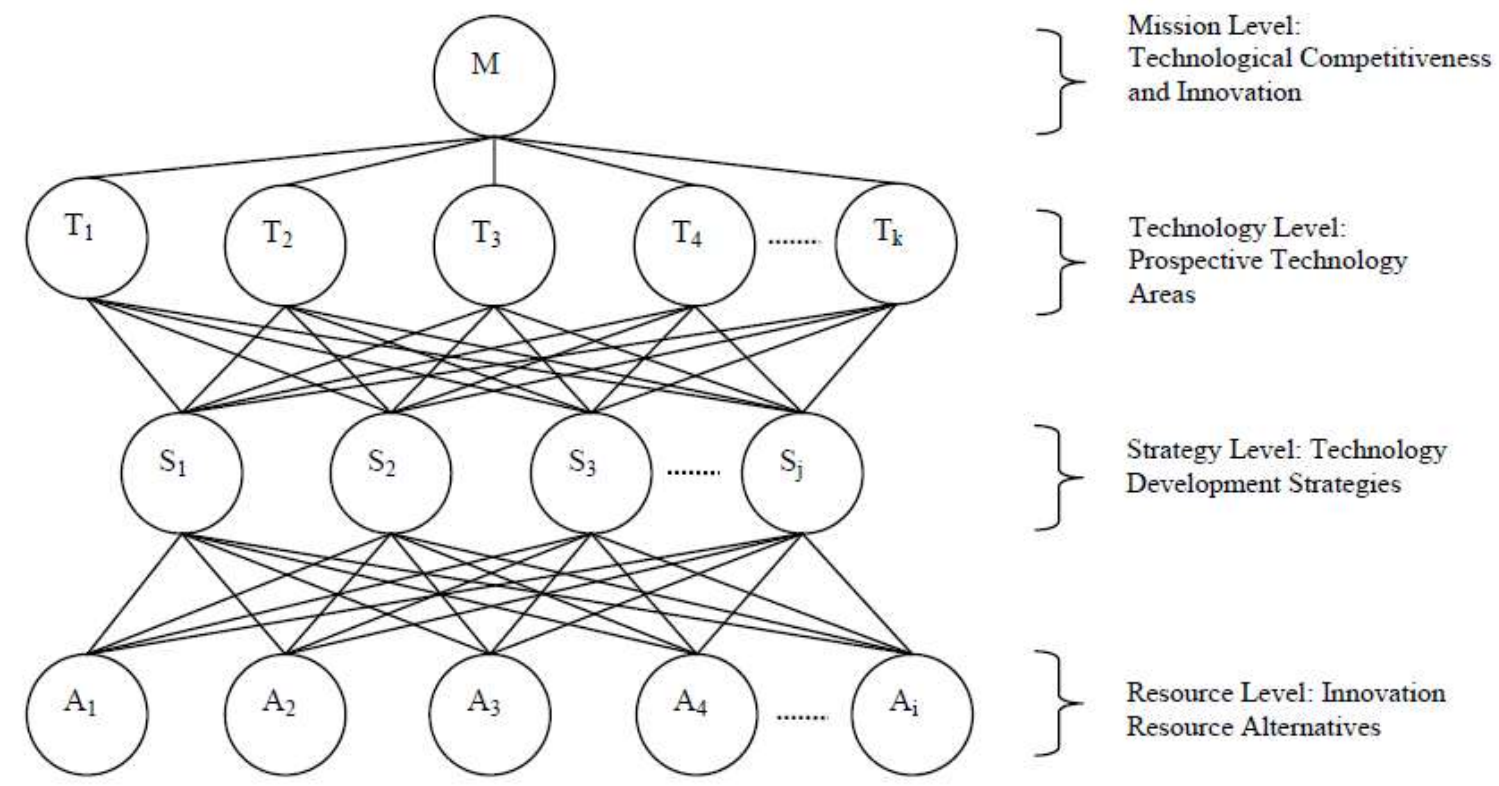

M: Mission - Technological Competitiveness and Innovation

$\mathrm{T}_{\mathrm{k}}$ : Prospective Technology Areas (k)

$\mathrm{S}_{\mathrm{j}}$ : Technology Development Strategies (j)

$\mathrm{A}_{\mathrm{i}}$ : Innovation Resource Alternatives (i)

$$
\begin{aligned}
& \mathrm{k}=1,2,3 \ldots \mathrm{K} \\
& \mathrm{j}=1,2,3 \ldots \mathrm{J} \\
& \mathrm{i}=1,2,3 \ldots \mathrm{I}
\end{aligned}
$$

Figure 8 Framework for Technological Competitiveness and Innovation (Chan et al. 2013)

Another important work validating the HDM approach was done in the field of technology assessment for energy efficiency programs in Pacific Northwest (Iskin and Daim 2014). The chainwise paired comparison method was utilized at the program alternative level due to the large number of program alternatives 
(Iskin and Daim 2014). The model was based on Research Institute for Sustainable Energy "RISE" research model. RISE model was developed by Kocaoglu and Daim and aimed to provide multi perspective assessment of various energy technologies from technical, economic, environmental, social and political perspective.

In semiconductors industry, HDM and sensitivity analysis were used for strategic assessment of the overall competitive success (Chen et al. 2009). With that in mission, the second level of hierarchy was the list of competitive goals, followed by technology strategies leading to the technology alternatives.

Another interesting research with application in semiconductors industry was performed to determine innovativeness of a company (Phan and Kocaoglu 2014). The methodology consisted of hierarchical decision model, evaluation of indicators and innovativeness. This research also implements the concept of desirability curve, the measure and representation of usefulness or 'goodness' of those values to the decision maker.

In agriculture with a particular case study of nanotechnology, HDM was used by researchers Gerdsri and Kocaoglu to develop national emerging technology strategy and policy supporting sustainable economy (Gerdsi and Kocaoglu 2008; Gerdsri and Kocaoglu 2009). The model consisted of four levels (mission, objectives, technological goals and research strategies (Gerdsi and Kocaoglu 2008). The research was directed to help policy makers establish R\&D strategy for any emerging technology according to the country's mission. The 
researchers consider HDM model and its results a decision support tool contributing to the R\&D strategy development of nanotechnology for Thailand's agriculture sector.

A recent paper used HDM for selecting medical hardware (cochlear implant device) from the patient's perspective for a real-world decision and demonstrates that it's a sophisticated decision-making tool for complex patientbased medical decision-making (Anderson and Jahromi 2016). High-level categories for the hierarchical model included performance, patient safety, usability and technical issues.

\subsection{Hierarchical Decision Model (HDM)}

Hierarchical Decision Model (HDM) developed by Dr. Kocaoglu, gives an opportunity to look at the program under consideration as a network of relationships among decision hierarchies, quantified by subjective judgments of experts in a systematic process so as to provide a sound basis for those complex evaluations (Kocaoglu 1983). HDM is widely used in Engineering and Technology Management discipline and has gained popularity in various industries (Turan et al. 2009, Fenwick et al. 2009; Kodali et al. 2009; Angst et al. 2010).

Multi-criteria decision tools like Saaty's Analytic Hierarchy Process (AHP) (Saaty 1977) and Hierarchical Decision Model (HDM) (Kocaoglu 1983) have some important steps in the application process: 
1. Structuring the decision problem into levels consisting of objectives and their associated criteria

2. Eliciting decision maker's preferences through pairwise comparison among all variables at every hierarchical level of the decision model

3. Processing the input from the decision-maker and calculating the priorities of the objectives

4. Checking consistency of the decision maker's responses to ensure logical and not random comparison of the criteria.

\subsection{Generalized Hierarchical Decision Model}

The generalized HDM for assessment of technology adoption potential is shown in Figure 9 and consists of four levels: mission statement; model perspectives, criteria and model alternatives. 


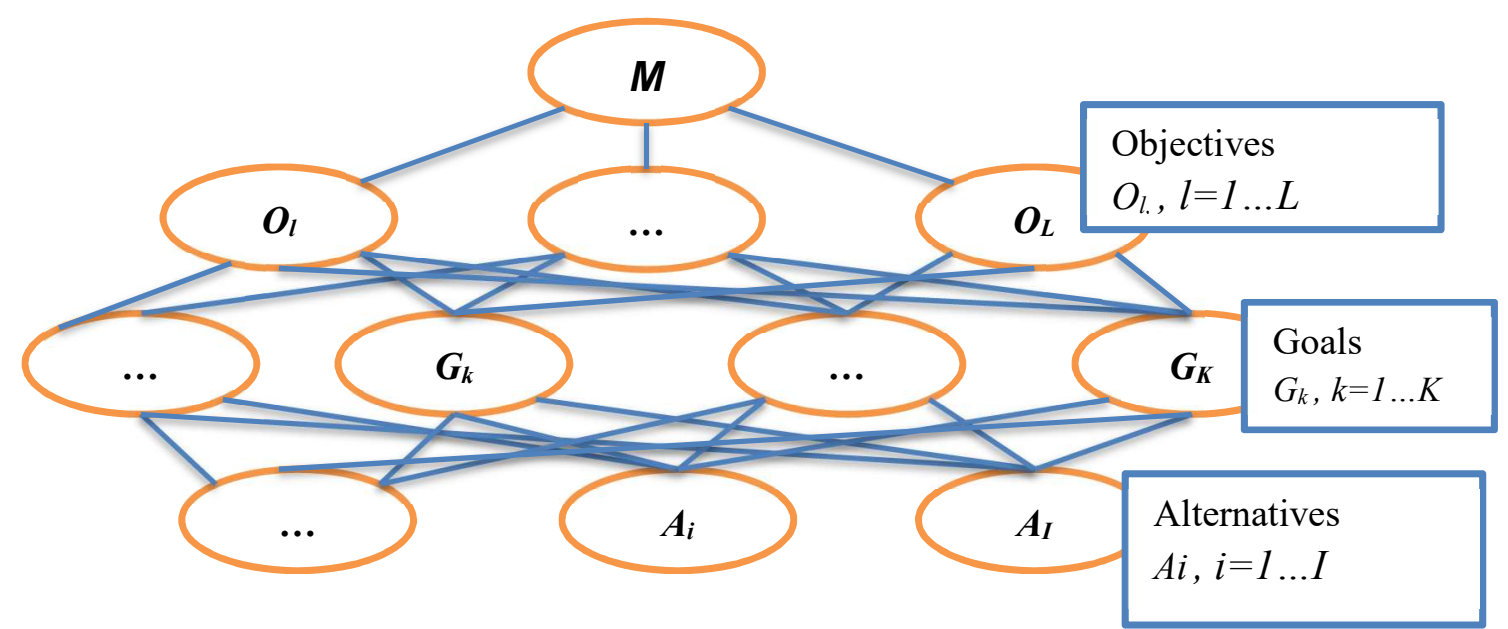

Figure 9 Generalized hierarchical decision model with 4 levels of hierarchy

- Mission: Assessment of technology adoption potential

- Objectives: $O$, with $I=1, \ldots, \mathrm{L}$

- Goals: $G_{k}$, with $\mathrm{k}=1, \ldots, \mathrm{K}$

- Alternatives: $A_{i}$, with $i=1, \ldots$, I

- $\mathrm{C}^{\mathrm{O}-\mathrm{M}}$ is the relative contribution of the $\mathrm{L}^{\text {th }}$ objective to the mission

- $\mathbf{C}_{\mathbf{k l}}{ }^{\mathrm{G}-\mathrm{O}}$ is the relative contribution of $\mathrm{k}^{\text {th }}$ goal to the $\mathrm{L}^{\text {th }}$ objective

- $\mathrm{C}_{\mathrm{ik}}{ }^{\mathrm{A}-\mathrm{G}}$ is the relative contribution of the to the $\mathrm{k}^{\text {th }} \mathrm{goal}$

- $\mathrm{C}_{\mathrm{il}}^{\mathrm{A}-\mathrm{O}}$ is the relative contribution of the $\mathrm{i}^{\text {th }}$ alternative to the $\mathrm{L}^{\text {th }}$ objective

- $\mathrm{Ci}_{\mathrm{i}}^{\mathrm{A}-\mathrm{M}}$ is the overall contribution of $\mathrm{i}^{\text {th }}$ alternative to the mission

According to the Constant Sum method, one hundred points was assigned

to be divided between the pairs of the elements at the same level of the hierarchy. The overall relative contribution of the model alternative (wearable sensor products) (A) to the mission (M) is calculated by adding the sum of the products of all local contribution matrices between $\mathrm{M}$ and $\mathrm{A}$ as depicted in the equation below (Chen and Kocaoglu 2008): 


$$
C_{i}^{A-M}=\sum_{l=1}^{L} \sum_{k=1}^{K} C_{l}^{O-M} C_{k l}^{G-O} C_{i k}^{A-G}
$$

\subsection{Judgment Quantification}

Judgment quantification (quantification of expert judgments for data collection purposes) is performed at different levels in the decision hierarchy. Pairwise comparison method is employed for judgment quantification.

In HDM, a variance-based approach is used for the inconsistency calculations and $10 \%$ limit is recommended on it in the Constant Sum Method (CSM). While the HDM approach is similar to Saaty's Analytic Hierarchy Process, the computational phase uses the Constant Sum Method instead of the Eigenvectors (Kocaoglu 1983). As explained by Dr. Kocaoglu, in the hierarchical decision process, the problem is considered as a network of relationships among major levels (impact, target and operational) of hierarchy, with multicriteria objectives at the top leading to multiple benefits and at the bottom - multiple outputs resulting from multiple actions (Kocaoglu 1983).

The constant-sum method (Kocaoglu 1983) consists of the following:

1. $n(n-1) / 2$ are randomized for the $n$ elements under consideration.

2. The decision-makers distribute a total of 100 points between elements with respect to each other. (It they are of equal importance both elements get 50 points, if one is four times higher/more important with respect to another, the allocation will be 80 to 20 points etc. 
3. The data is written into matrix $A$, through comparing column elements with row elements.

4. Matrix $B$ is obtained by taking the ration of comparisons for each pair from Matrix A.

5. Matrix $C$ is constructed through division of each element in a column of Matrix $B$ by the element in the next column.

Element $d$ is assigned a value of 1 and the calculation of other elements is performed by ratios as the mean of each column in Matrix $\mathrm{C}$.

\subsection{Inconsistency Measure}

When pairwise comparisons have some inconsistencies, the consistency measure is calculated in order to determine the level of internal consistency in the comparisons of the judgments (Ra 1988).

The inconsistency is specified through nonzero standard deviations under the Matrix C columns (Kocaoglu 1983). Matrix C (discussed above) should be repeated for all $n$ ! orientations of the $\mathrm{n}$ elements.

With normalized values calculated for each orientation, the final value for each element (the mean of the $n$ ! relative values) is determined and the variance in the relative elements distribution is used for internal inconsistency measure.

The formula of inconsistency measure (Kocaoglu 1983) is shown below:

$$
\text { Inconsistency }=\sqrt{(1 / n) \sum_{i=1}^{n}(1 / n !) \sum_{j=1}^{n !}\left(r_{s u b_{i}}-r_{i j}\right)^{2}}
$$


where $r_{i j}$ is the relative value of element $\mathrm{i}$ in $\mathrm{j}^{\text {th }}$ orientation;

$r_{s u b_{i}}$ is the average subjective value of element $\mathrm{i}$.

$$
r_{s u b_{i}}=(1 / n !) \sum_{j=1}^{n !} r_{i j}
$$

A recent study by Abbas (2016) established consistency thresholds linked to for HDM's judgment quantification method. The measure is linked to number of decision variables and alpha ( $\alpha$ ) level for the purposes of evaluating the soundness and validity of the judgment. The inconsistency measure proposed by Abbas (2016) is the square root of the sum variances and defined as the Root of the Sum of Variances (RSV) of the $\mathrm{n}$ decision elements:

$$
R S V=\sqrt{\sum_{i=1}^{n} \sigma_{i}^{2}}
$$

where:

$\sigma_{i}^{2}$ is the variance of the mean of the $\mathrm{i}^{\text {th }}$ decision element, $\mathrm{n}$ is the number of decision elements:

$$
\sigma_{i}=\sqrt{\frac{1}{n !} \sum_{j=1}^{n !}\left(x_{i j}-\bar{x}_{i j}\right)^{2}} \quad \forall i=1, \ldots, n
$$

where:

$x_{i j}$ is the normal relative value of the variable $\mathrm{i}$ for the $\mathrm{j}^{\text {th }}$ orientation in $\mathrm{n}^{\text {th }}$ factorial orientations; 
$\bar{x}_{i j}$ is the mean of the normalized relative value of the variable I for the $\mathrm{j}^{\text {th }}$ orientation:

$$
\bar{x}_{i j}=\frac{1}{n !} \sum_{j=1}^{n !} x_{i j}
$$

\subsection{Expert Disagreement}

Sometimes experts may disagree on the relative importance of the indicators, and/or the relative importance of the sub-factors in the model. Disagreements are natural and are described in research (Pandejpong and Kocaoglu 2002; Légaré et al. 2010; Turan et al. 2009; Phan 2013). The level of disagreement can be examined with the coefficient of intraclass correlation, with measures the degree to which the judges $(X)$ are in agreement with one another on the relative importance of subjects $(n)$ :

$$
r_{i c}=\frac{M S_{B S}-M S_{r e s}}{M S_{B S}+(k-1) M S_{r e s}+\frac{k}{n}\left(M S_{B J}-M S_{r e s}\right)}
$$

where:

$\mathrm{MS}_{\mathrm{BS}}$ is the mean square between decision elements,

MSres is mean residual square,

$M S_{B J}$ is the mean square between experts,

$k$ is the number of experts,

$\mathrm{n}$ is the number of decision elements 


$$
\begin{aligned}
& M S_{B S}=\frac{S S_{B S}}{d f_{B S}} \\
& S S_{B S}=\sum_{i=1}^{n}\left[\frac{\left(\sum S_{i}\right)^{2}}{k}\right]-\frac{\left(\sum X_{T}\right)^{2}}{n k} \\
& d f_{B S}=n-1 \\
& M S_{\text {res }}=\frac{S S_{\text {res }}}{d f_{\text {res }}} \\
& S S_{\text {res }}=S S_{T}-S S_{B J}-S S_{B S} \\
& d f_{\text {res }}=(n-1)(k-1) \\
& S S_{T}=\sum^{X_{T}^{2}-\frac{\left(\sum X_{T}\right)^{2}}{n k}} \\
& M S_{B J}=\frac{S S_{B J}}{d f_{B J}} \\
& S S_{B J}=\sum_{j=1}^{k}\left[\frac{\left(\sum X_{j}\right)^{2}}{n}\right]-\frac{\left(\sum X_{T}\right)^{2}}{n k} \\
& d f_{B J}=k-1
\end{aligned}
$$

This research used PCM group disagreement index to examine group disagreements in expert panels and Hierarchical Cluster Analysis is used to identify experts and expert groups that are in conflict with the rest of the group and outline new clusters with regrouping of experts into new groups with similar judgments. If the group disagreement exceeds the threshold value of 0.1 , cluster analysis is used to analyze it. 
Below is the formula for the disagreement index for $\mathrm{j}$ experts for $\mathrm{n}$ decision variables in each panel:

$$
d=\sqrt{\frac{1}{m}} \sum_{j=1}^{m} \frac{1}{n} \sum_{i=1}^{n}\left(R_{i}-r_{i j}\right)^{2}
$$

where:

$$
\begin{aligned}
& R_{i} \text { - group relative value of the } \mathrm{i}^{\text {th }} \text { element, } \\
& \mathrm{m} \text { - the number of experts, } \\
& \mathrm{n} \text { - the number of decision variables, } \\
& r_{i j} \text { - mean relative value of the } \mathrm{i}^{\text {th }} \text { element for } \mathrm{j}^{\text {th }} \text { expert }
\end{aligned}
$$

Disagreements happen, since experts, due to their field of knowledge, perceptions, beliefs and experience in their field, might have different opinions in their criteria judgements. Disagreements are analyzed through clustering judgments into homogenous groups with common characteristics and analyzing their values as well as their impacts on the outcomes of the model.

\subsection{Sensitivity Analysis}

Sensitivity analysis (SA) is performed in order to determine the allowable perturbations of different levels of the hierarchical model. The effect of changes in priorities of the objectives or goals on the model outcomes is determined with utilization of sensitivity analysis. In addition to the inconsistency measures, that will be performed, a sensitivity analysis algorithm for HDM, developed by Dr. 
Chen and Dr. Kocaoglu will be engaged. The researchers propose two sensitivity coefficients (Chen and Kocaoglu 2008) to study robustness of the current decision and flexibility of the input values without changing the decision:

1. The operating point sensitivity coefficient (OPSC), defined as a the shortest distance from the current contribution value to the edges of its tolerance (dependent on the contribution's current value and directions of change)

2. Total sensitivity coefficient (TSC), which specifies that the shorter the tolerances of a decision element's contributions are, the more sensitive the final decision is to the decision element's variations.

According to the research performed by (Chen and Kocaoglu 2008), the authors conclude that performing sensitivity analysis for HDM can be useful in a number of ways:

1. visualization of the impact of changes at the policy and strategy levels on decisions at the operational level;

2. testing the robustness of the recommended decision;

3. identification of the decision's critical elements;

4. generating scenarios of possible rankings of decision alternatives under different conditions;

5. assisting the experts in reaching the consensus;

6. offering answers to the "what-if" questions. 
The sensitivity analysis approach has been developed particularly for HDM, based on extensive research and therefore will be applied in our research. Other approaches to sensitivity measures also exist (Chen and Kocaoglu 2008; Chen and Li 2011) and include:

- simulations approach (replacing the values in the local contribution matrix with probability distributions while calculating the expected value of ranks involving numerous simulation runs;

- mathematical deduction (used when simple-form expressions are introduced to describe the relationship between inputs and outputs);

- numerical incremental analysis (an iteration-based and data-dependent process - used in Expert Choice software).

Sensitivity analysis in HDM examines the effect of any changes in the criteria on the rankings of alternatives. Tolerance is "the allowable range, in which a contribution value can vary without changing the rank order of decision alternatives", according to Chen and Kocaoglu (2008).

For the perturbations at the objectives level, $P_{l *}^{O}$ representing the perturbations applied to one of the objectives $C_{l}^{O}$ where $\left(-C_{l^{*}}^{O} \leq P_{l^{*}}^{O} \leq 1-C_{l^{*}}^{O}\right)$, the original ranking of $A_{r}$ and $A_{r+n}$ will not change if

$$
\lambda \geq P_{l}^{o} \times \lambda^{o},
$$

where:

$$
\lambda=C_{r}^{A}-C_{r+n}^{A}
$$




$$
\lambda^{o}=C_{r+n, l *}^{A-O}-C_{r, l *}^{A-O}-\sum_{l=1 . l \neq l *}^{L} C_{r+n, l}^{A-O} \times \frac{C_{l}^{o}}{\sum_{l=1, l \neq l *}^{L} C_{l}^{o}}+\sum_{l=1, l \neq l *}^{L} C_{r, l}^{A-O} \times \frac{C_{l}^{o}}{\sum_{l=1, l \neq l *}^{L} C_{l}^{o}}
$$

According to theorem 1 Chen and Kocaoglu (2008), the highest rank will remain unchanged if the above condition is satisfied for all $r=1$ and $n=1,2 \ldots l$ 1. Also, the rank order of all $A_{i}$ will be unchanged if the above condition is satisfied for all $\mathrm{r}=1,2 \ldots \mathrm{I}-1, \mathrm{n}=1$. Allowable range of perturbations on $C_{l}^{O}$ to keep the current ranking is $\left[\delta_{l-}^{o}, \delta_{l+}^{o}\right]$. The operating point sensitivity coefficient and total sensitivity coefficient are calculated according to the formulas below:

$$
\begin{gathered}
\operatorname{OPSC}\left(O_{l}\right)=\operatorname{OPSC}\left(C_{l}^{O}\right)=\operatorname{Min}\left\{\left|\delta_{l-}^{o}\right|,\left|\delta_{l+}^{o}\right|\right\} \\
\operatorname{TSC}\left(O_{l}\right)=\operatorname{TSC}\left(C_{l}^{O}\right)=\left|\delta_{l+}^{O}-\delta_{l-}^{O}\right|
\end{gathered}
$$

\subsection{Expert Panel Design}

A panel of experts are people distinguished in the field of management of IT and healthcare, who are aware of the wearable sensor technologies and are able to rate them according to the criteria. The snowballing method was used to come up with an acceptable number of experts (Kocaoglu 1983). The experts are distinguished in their fields. Expertise, according to Olson and Rueter, is primarily a skill of recognition or 'seeing' old patters in new problems, organizing the concepts in the knowledge bases with much more depth and central association (Olson and Ruetger 1987). There are two classes of methods that reveal experts knowledge: direct (asking an expert to report on knowledge he can directly articulate) and indirect (not relying on the expert's abilities to articulate the 
information, but instead collecting other behaviors and using analyst's inferences about what the expert 'must have known' according to his/her response) (Olson and Ruetger 1987).

\subsubsection{Direct methods}

- Interviews guidelines to be followed:

- Enlisting the expert's cooperation

- Asking free-form questions at the start and then narrowing them toward the end of the interview

- Not imposing own understanding on the expert

- Limiting the sessions to coherent tasks while recognizing fatigue and attention limits

- Questionnaires have advantage of being a very efficient way to gather information: the experts can fill them out in a relaxed atmosphere. Questionnaires are very useful in discovering the object of the domain, uncovering relationships and determining uncertainties. While interviews are not effectives in estimating probabilities, pre-formatted response scales can yield much more accurate estimates. The two preferred formats are

o the bar on which the point reflecting uncertainty;

- a five point verbal scale

- Observation of a task performance (recording an expert's performance either by watching or using a recording device, where the first method may 
suffer from time pressure and observer bias and the latter one depends on expert's ability to recall the reasons underlying his/her performance).

- Protocol analysis (In additional to observational techniques, the expert is asked to "think out loud" while performing a task, afterwards the protocol is analyzed. Disadvantages: not good for tasks for which there is no natural verbalization, or the process of "talking out loud" may interrupt expert's performance.

- Interruption analysis, where the expert can be interrupted by the observer, if observer needs to clarify something.

- Drawing closed curves (The expert is asked to draw the related objects in a closed curve).

- Inferential flow analysis is a variant on the interview, where answers to particular questions about causal relations are used to build up a causal network among concepts or objects in the expertise domain.

\subsubsection{Indirect methods}

- Multidimensional scaling (MDS) - a technique that should be used only in a case, where it is assumed that data came from stored representations of physical n-dimensional space.

- Johnson hierarchical clustering assumes (contrasting from MDS) merely that an item is or not a member of a cluster with judgments being a function of the number of nested clusters two items have in common. 
- General weighted networks (the expert gives symmetric distance judgments on all possible pairs of objects

- Ordered trees from recall (the technique assumes that objects belong to a cluster or not, with a notion that people recall all items from a stored cluster before recalling items from another cluster)

- Repertory grid analysis consists of:

○ initial dialog with the expert

- a rating session

$\circ$ analyses that both cluster the objects and the dimensions on which the items were rated

\subsubsection{Minimizing bias}

In addition of having knowledge and understanding the issues with healthcare management, and explicitly technology adoption of wearable sensor products, we minimized the bias by making sure that the experts chosen for panels have no vested interest in the any particular outcome of the decisionmaking process and research outcomes and we will also create a balanced representation of researchers and administrators (Kocaoglu 1983). The experts were given an opportunity to get acquainted with the research background information, the measurement procedure and other documentation or clarifying information prior to making their judgments. While the panel meetings were selected, other methods were available and were considered as discussed by Olson and Ruetger. The experts are asked to perform pairwise comparisons in 
Qualtrics with the slider, distributing 100 points between the pairs of variables of each level of the decision hierarchy.

\subsubsection{Focus and expertise of expert panels}

Data collection is carried out in expert panels. The proposed research consists of seven expert panels.

- Expert panel 1 - Main multiple perspective criteria

The first panel is for main criteria level of the hierarchy and consists of executive managers, leaders in neurosurgery and orthopedics people, who are in higher managerial positions in the healthcare field and are able to compare highlevel perspectives for assessment of technology adoption potential of wearable sensor products in wearable sensor products in neurosurgery and orthopedics. Those experts have a broad understanding of the objectives provided and are able to assess their importance to the main mission and in relation to each other.

- Expert panel 2 - Financial perspective criteria.

Expert panel 2 analyzed financial criteria. The experts for this panel have significant experience in the departmental decision making including financial considerations, leadership in neurosurgery and orthopedics department as well as hospital administration.

- Expert panel 3 - Technical criteria.

The experts in this panel have a broad knowledge and experience of technical characteristics of the healthcare field and particularly wearable sensor 
technologies. This panel consists of engineers, technologists, hospital IT managers and specialists.

- Expert panel 4 - Organizational criteria.

Expert panel 4 focuses on assessing importance of organizational criteria like management support, standardization and tech support. Those experts have significant experience in project management, personnel management, technology management, human resource allocation and understand specifics of hospital care. The experts come from healthcare organizations and academia.

- Expert panel 5 - Patient criteria.

Experts of panel 5 are doctors, nurses and IT specialists with experience in the field of neurosurgery and orthopedics. They have the knowledge and experience to assess Clinical Benefits for Patients criterion as well as Patient Experience and Privacy / Security criteria.

- Expert panel 6 - Interpersonal criteria.

Since interpersonal criteria focuses on issues of communication and sharing, the main expertise is provided by doctors, nurses and IT specialists. This group of experts has experience in doctor-patient; nurse-patient and doctor-nurse communication patterns as well have a good understanding of issues of sharing information.

- Expert panel 7 - Alternatives level.

This panel consists of engineers, technologists, project/program managers, people familiar with impacts of software/hardware/wearable products in 
healthcare, biosensors, particularly in the neurosurgery and orthopedics and could evaluate wearables with respect to the criteria provided.

Table 20 Focus and required expertise of expert panels for this research

\begin{tabular}{|l|l|l|}
\hline Panel \# & \multicolumn{1}{|c|}{ Focus } & \multicolumn{1}{c|}{ Required expertise } \\
\hline Panel 1 & Multiple perspective criteria & $\begin{array}{l}\text { Executive managers, leaders in } \\
\text { neurosurgery and orthopedics } \\
\text { departments and academia }\end{array}$ \\
\hline Panel 2 & Financial criteria & $\begin{array}{l}\text { Strategic directors, VPs, hospital } \\
\text { finance administrators \& academia }\end{array}$ \\
\hline Panel 3 & Technical criteria & $\begin{array}{l}\text { Engineers, technologists, hospital } \\
\text { IT specialists, leaders in } \\
\text { neurosurgery and orthopedics and } \\
\text { academia }\end{array}$ \\
\hline Panel 4 & Organizational criteria & $\begin{array}{l}\text { Project managers in the hospital, } \\
\text { human resource managers, } \\
\text { neurosurgeons, orthopedic } \\
\text { surgeons, academia experts }\end{array}$ \\
\hline Panel 5 & Patient criteria & Doctors, specialists in spine care \\
\hline Panel 6 & Interpersonal criteria & Doctors, nurses, IT specialists \\
\hline Panel 7 & Alternatives level & $\begin{array}{l}\text { Engineers, technologists, project / } \\
\text { program managers }\end{array}$ \\
\hline
\end{tabular}

\subsection{Research Validation}

The model will be validated by the panels of experts from healthcare and academia. Validation by definition is a test of whether the model is an adequate (sufficient) representation of the elements and their relationships of the actual systems it's referring to with their underlying importance to planned experiments (Greenberger et al. 1976; Labys 1982; Paré and Sicotte 2001). Validation is 
necessary in research for ensuring rigorous standards and adding credibility to a model constructed and studies by a researcher (Robert Wood Johnson Foundation 2010). Validation is the correspondence of the model to the fundamental process that is being modeled. Labys (1982) outlines three important items of validity:

- Statistical significance of the parameter configuration of the structure;

- The correspondence of ex post and ex ante estimated model's variable values to actual data;

- Sensitivity of the model

Greenberger et al. (1976) stated that there is no uniform procedure for model validation and it should be emphasized that since all models are simplifications of the reference systems they are never entirely valid i.e. fully supported by objective truth (Greenberger et al. 1976; Labys 1982).

Three major types of validity (Table 21) will be engaged for the purposes of this research. Construct validity as previously described will be done by a select group of experts to verify the structure of the model. Content validity will test the readiness of the instruments to gather data from respondents. Criterionrelated validity will be done after the study through review of the results by the experts and examining whether they are acceptable. Model generalization is discussed by the experts. 
Table 21 Types of validity for the proposed research

\begin{tabular}{|l|l|l|l|}
\hline Validity & \multicolumn{1}{|c|}{ Description } & \multicolumn{1}{|c|}{ Method } & When \\
\hline $\begin{array}{l}\text { Content } \\
\text { validity }\end{array}$ & $\begin{array}{l}\text { Degree to which a measure } \\
\text { represents a given domain } \\
\text { of interest and will test the } \\
\text { readiness of the instruments } \\
\text { to gather data from } \\
\text { respondents. }\end{array}$ & $\begin{array}{l}\text { Expert evaluation, } \\
\text { and literature } \\
\text { review }\end{array}$ & $\begin{array}{l}\text { During the } \\
\text { model } \\
\text { development }\end{array}$ \\
\hline $\begin{array}{l}\text { Construct } \\
\text { validity }\end{array}$ & $\begin{array}{l}\text { Degree to which a proposed } \\
\text { research approach complies } \\
\text { with its underlying theories. } \\
\text { (Verification of the structure } \\
\text { of the model.) }\end{array}$ & Expert evaluation & $\begin{array}{l}\text { After the } \\
\text { model } \\
\text { development }\end{array}$ \\
\hline $\begin{array}{l}\text { Criterion- } \\
\text { related } \\
\text { validity }\end{array}$ & $\begin{array}{l}\text { Degree of effectiveness of a } \\
\text { model in predicting real life } \\
\text { phenomenon. (Review of the } \\
\text { results by the experts and } \\
\text { examining whether they are } \\
\text { acceptable). }\end{array}$ & Expert evaluation & After the \\
analyses
\end{tabular}




\section{CHAPTER 4. CASE STUDY BACKGROUND}

This chapter contains literature research pertaining to the case application, development of alternatives of the model, mobile and wearable applications in healthcare and their assessment.

\subsection{Mobile Healthcare Systems}

Gurses and Xiao (2006) provided systematic review of literature on multidisciplinary rounds to design information technology. According to their study, which was also illuminated by Cresswell and Sheikh, they suggest that the positive impact on communication and collaboration in hospital care could be achieved through a range of attributes of IT, particularly use of mobile technologies to increase flexibility, checklists and supporting informal communication (Gurses and Xiao 2006; Cresswell and Sheikh 2012). Mendonça et al. (2004) in their study on mobile information and communication for health care noted that they anticipate hand-held wireless applications will improve patient care by reducing proximal causes of medical errors and other adverse events. Other researchers indicate that emergence of mobile device channels as new technology-driven channels adds value and enhances channel flexibility (Viswanathan 2005; Kulendran et al. 2014), others comment on challenges of simplification of the software use and design of easy-to-use software for knowledge workers and cast doubt on any particular "silver bullet" technology like mobile computing, voice recognition, tablet computers, will dramatically impact the use of the software (Miller and Sim 2004). However, recent Gartner report 
Predicts 2012: Mobile and Wireless Technologies Rise indicates that mobile and wireless technologies continue to become more mainstream and impactful, less of a stand-alone technology and will integrate more into other technologies (Dulaney et al. 2011). Researchers (Wu et al. 2007) presented a revised technology acceptance model to examine mobile healthcare systems (MHS) acceptance by physicians and their results indicated that compatibility, perceived usefulness and perceived use significantly affect healthcare professional behavioral intent. They also suggested that careful attention needs to be paid to user requirements analysis to determine the user expectations for mobile healthcare application content (Wu et al. 2007).

In another paper Wu et al. (2009), while looking at healthcare technology management competency and impacts on IT healthcare partnership development, describe healthcare technology integration $(\mathrm{HTI})$ as the ability to visualize the ways in which various kinds of technologies (like mobile/ubiquitous computing) can contribute to healthcare organization performance and help synergize information systems, healthcare professionals' knowledge and organization performance.

\subsection{Importance of Patient-Oriented Intelligible Systems}

One group of researchers was very successful in interface design, because they were able to audit physician interactions with the clinical information system and analyze usage patterns and gain objective data about performance (Melles et al. 1998). In another study, the researchers learned that 
providers want EHR that requires less complexity - a minimum of keystrokes, mouse clicks, scrolling, window changes etc. While the flexibility that accommodates various data entry styles has been built in, it could complicate data extracting accuracy and efficiency (Roth et al. 2009). Some researchers even noted that TAM is "an analytical simplification of how functionality and interface characteristics relate to adoption decisions" (Melas et al. 2011).

Efficiency in itself could have positive effects on cost through improved productivity, paper reduction, reduced transcribing, drug utilization and improved lab tests, care coordination, reduction in administrative time (Wong et al. 2003; Jung 2006) and improved patient safety and regulatory compliance (Bates et al. 1998; Agarwal 2002; Jung 2006). Zhou et al. (2012) notes that a sophisticated graphical user interface is critical for user friendly rule authoring environments (RAEs), addressing individual end user needs and expectations. In a study of lessons learned from health information systems implementation in seven countries, the researchers outlined that systems GUI quality, feature functionality, project management, procurement and users' previous experience affect outcomes of IT implementation (Ludwick and Doucette 2009). Gorla et al. (2010) stress three important points about excellence in IS quality:

- The value of information systems may be realized through improving profit margins for the firm, following best practice software standards in the industry, providing easy-to-use and useful applications and designing of easily maintainable software. 
- The quality of information systems represents designing systems that conform to the end users' information requirements and adhere to industry standards.

- Customer expectation of IS quality is met through offering of appealing, user-friendly interfaces, entertaining user requests for changes, and satisfying the stakeholders of the IS.

Convenient interface that is easy to use and adjust to is possibly one of the most and first noticeable user-friendly features of the system (Pagemill Partners 2014). However, the user might not require a fancy interface and may need an interface that fits the need of the clinic. A user interface that is poorly designed with fragmented screens and multiple sign-ins can increase computer time and also lead to dissatisfaction (Furukawa 2011). Interface is a discussed topic in research and is often mentioned in phrases as "interface design" or “interface design quality" (Ludwick and Doucette 2009; Ayatollahi et al. 2009; Degoulet et al. 1995; Becker et al. 2011; Alper and Olson 2010; Cresswell and Sheikh 2012; Despont-Gros 2005; Yusof et al. 2008; Melas et al. 2011; Valdes et al. 2004; Davis 1989; Moores 2012).

\subsection{Preventative and Integrated Care Systems}

Increased emphasis on preventive measures and early detection of diseases, primary care, intermittent healthcare services and continuity of care is prevalent in our ever-changing healthcare domain (Tsiknakis et al. 2002; Intille 2004; Dhawan et al. 2015). Information and communication technologies are 
taking lead in this dynamic environment with the need for improved quality of healthcare services and costs control and movement towards shared and integrated care (Tsiknakis et al. 2002; Yamamoto et al. 2008). The need for other software and particularly patient care systems to be able to seamlessly integrate with EHR system is a part of the coordinated care system. (McGinn et al. 2011) notes that inadequate interfacing with other IT systems was perceived as a barrier by users according to the literature review and in some cases led to negative outcomes.

Several researchers state that integration of heterogeneous IS was always problematic in healthcare organizations, therefore those organizations are looking to increase their functional capabilities and decrease integration costs (Khoumbati et al. 2006).

Another Canadian group of researchers proposed a framework to address the issues of data and knowledge interoperability by adopting healthcare and data mining modeling standards (Kazemzadeh et al. 2010). Their healthcare environment involving a guideline-based clinical decision support system and healthcare framework for operability of data and mined knowledge with shaded areas corresponding to contributions of the researchers are shown below (Kazemzadeh et al. 2010).

According to survey of hospitals and integrated delivery networks, conducted by HIMSS Media on behalf of Philips Healthcare, EMR interoperability was most important in choosing clinical technologies (44\%) and the most 
important attributes of a successful clinical informatics vendor were:

interoperability with existing EMR (42\%), deep clinical knowledge (32\%) and interoperability with existing clinical information systems (22\%) (Philips 2013).

\subsection{Biometric Technologies}

Smartphones gained sophistication over the years with an array of sensors either built into the phone, or with the capability to be affixed to the phone, in order to collect biometric and other data about consumers or patients to support remote health monitoring (Dunbrack 2014; MarketsandMarkets 2012; Munos et al. 2016; Neal and Woodard 2016; Bhattacharya 2015; Crocker and Lewis 2010; Guennoun et al. 2008). Some bright examples of those technologies include:

- Ginger.io -- sensor passively collects information from the consumer tracking consumer interactions with the device - frequency, length, and timing of phone calls and texts and movement through the incorporation of GPS. for example, less movement or less communication with friends and family may indicate that the person is isolating himself/herself and is possibly depressed. (Dunbrack 2014)

- AliveCor System - heart monitor device snaps to the smartphone and can record, display, store and transfer single-channel electrocardiogram (ECG) rhythm steps. Real-rhythm assessments could be provided to patients and doctors (Dunbrack 2014). 
- The Smartphone Physical - developed by John Hopkins medical student, showcases enables a variety of medical devices for a collection of quantitative or qualitative data, clinically relevant for physical evaluation, for example. body weight, blood pressure, heart rate, blood oxygen level, visual acuity, optic disk and tympanic membrane images, pulmonary function values, electrocardiogram, heart and lung sounds, and ultrasound visualization (carotid artery imaging) (Dunbrack 2014). In another example, researchers (Feied et al. 2004) also stressed the needs of medical community of biometric systems for identification and authorization. Having tested those systems based on fingerprints, hand morphology, facial structure, voiceprints and other contact and non-contact technologies, they have identified iris scanning as the most suitable technology meeting the healthcare community needs in identification and authorization category (Feied et al. 2004).

\subsection{Wearable Healthcare Market}

According to IDTechEx, wearable technology market has a high growth potential with $\$ 20$ billion in 2015 to $\$ 70$ in 2025 . Wearable device market will grow to $\$ 41$ billion by 2020 (Soreon Research 2014). The global medical wearable electronics market is expected to surpass $\$ 7.9$ billion by 2021 (Mordor Intelligence 2017). The emergence of wearables is driven by:

- A shift to disease prevention

- Personalization of medical care 
- Importance of medical standards

- New players (software and hardware companies) changing healthcare industry dynamics.

Emergence of wearables is bringing deep transformation to the healthcare sector. Wearable technology is a large market, transforming and evolving healthcare delivery model, where care is being brought to the patient (Munos et al. 2016).

Digital technology helps boost doctor-patient relationship by

- Providing patient diagnostic information

- Promoting self-management of chronic diseases

- Increasing interaction between patients and clinicians

- Encouraging teamwork in caregivers.

\subsection{Pervasive Care}

The paradigm of the $6 \mathrm{Ps}$ (participation [of the whole nation], prevention [of illness], [early disease] prediction, pre-emptive [treatment] for realization of pervasive and personalized healthcare) has been discussed in literature with the emphasis of introduction of wearable medical devices (miniaturized, integrated, networked, digitalized and standardized) (Poon and Zhang 2008).

Chinese researchers Carmen C.Y. Poon and Yuan-Ting Zhang have listed a number of schemes that could ensure delivery of low-cost and quality healthcare, where implementation of wearable medical technologies is intended for each layer (Poon and Zhang 2008). Those schemes are: 
1. introduction of preventative healthcare strategies;

2. development of advanced technologies for reduction of labor and equipment costs;

3. invention of new technologies for more accurate diagnosis and therapy;

4. streamlining of diagnosis process;

5. balanced contribution system;

6. elimination of unnecessary healthcare services (Poon and Zhang 2008). Sarasohn-Kahn (2013) gave various examples of pervasive care with applications of integrated and passive sensors managing conditions like asthma, Alzheimer's, ingestion of medications, sleep disorders, diabetes etc.

\subsection{Wearable Medical Systems}

Modern healthcare system should be moving towards ubiquitous healthcare i.e. healthcare at any time and any place and such system needs support of a wearable system for continual tracking and measurement of biological signals of patients through wearable sensors with useful and meaningful feedback for medical personnel.

Wearable systems for patient monitoring consist of three main modules:

1. sensing and data collection hardware (for physiological and movement data);

2. communication hardware and software (for relaying data to a remote center); 
3. data analysis methods and techniques (for extracting clinically-relevant information (Patel et al. 2012).

Many chronic diseases and conditions (cardiovascular or Parkinson's) rely on prompt responses to some warning signals or other subtle changes in patients condition and those could be monitored and tracked by wearable medical devices (Sarasohn-Kahn 2013; Soh et al. 2015). Healthcare providers seek costeffective accessible care delivery systems, shifted towards prevention, early detection, early diagnosis, and early treatment of diseases while wearable medical systems are recognized as enabling technology, where patient's health could be monitored on a continual basis, providing relevant information to the medical professionals with alarms for adverse conditions and unusual readings (Teng et al. 2008; Redmond et al. 2014; Wannenburg and Malekian 2015; Sarasohn-Kahn 2013; Shany et al. 2012; Soh et al. 2015).

There is some research on construction of wearable context aware systems for ubiquitous healthcare (Pantelopoulos and Bourbakis 2010; Kang et al. 2006; Redmond et al. 2014). Researchers Kang et al. (2006) proposed a wearable context aware framework focused on sending information from wearable sensors to healthcare service entities, which acts as interoperability middleware between sensor makers and healthcare service providers. The authors demonstrate some wrist type and chest belt type wearable systems. The importance of improved interoperability standards for information exchange, plug 
and play device interactions and configurability has been discussed by other researchers (Warren et al. 2005; Teng et al. 2008).

Xiao-Feng Teng et al. (2008) provide methodological review of wearable medical systems, wearable sensing of physiological parameters and biochemical variables (cardiac activity, blood pressure, blood oxygen saturation, respiration, biochemical measurements), body area network, standards and other enabling technologies and applications of wearable medical systems for $p$-Health (or public health), particularly for Parkinson's disease and epilepsy through the use of accelerometers and gyroscopes. The authors also suggest the advantac6ges of implementation of wearable medical devices for the new $p$-Health paradigm of disease management:

1. prediction of acute events with long-term monitoring and analysis;

2. instant diagnosis of events with connection to the emergency care;

3. telemedicine intervention (more optimal disease management with possible reductions in treatment duration)

4. rehabilitation at home for maximizing of functional outcomes (Teng et al. 2008).

The researchers divide applications of wearable systems into three categories:

- Management of personal health conditions;

- Management of cardiovascular conditions;

- Management of neurological conditions (Teng et al. 2008). 
Another group of researchers remarked on the lack of interoperability, lack of sync and obstructive policy and proposed a collaborative policy design framework for enhancing development of wearables and guiding interdisciplinary collaborators of the wearable computing market (Baker et al. 2015). In addition to the physical parameters of the wearable technology, they discuss the context of use, privacy and security requirements, conspicuousness, observers' experience and sensory requirements.

\subsection{Medical Sensors and Targeted Applications for Patient Monitoring}

Various researchers proposed targeted applications of wearable systems, innovations and monitoring frameworks for accessible mobile health (Atallah et al. 2013; Aziz et al. 2007; Aziz et al. 2011; Ciuti et al. 2015; Clifton et al. 2013; Condell et al. 2011; Lane et al. 2010 ; Mehta et al. 2012; Möller 2015; Nagy et al. 2006; Ogunduyile et al. 2013; Redmond et al. 2014; Shany et al. 2012; Slade Shantz and Veillette 2014; Escayola et al. 2009; Klingbeil et al. 2010). Accelerometers and gyroscopes proved to be accurate body-mounted measurement systems (Mayagoitia et al. 2002; Möller 2015, Ogunduyile et al. 2013; Scheirey et al. 2013; Tobergte and Curtis 2013; Van Lummel et al. 2013; Zijlstra et al. 2010; Atallah et al. 2013; Aziz et al. 2007; Aziz et al. 2011; Ciuti et al. 2015). Researchers from Greece presented a mobile health real-time monitoring framework with wearables (Stavropoulos et al. 2015). Their HealthMon framework provides a mobile monitoring solution and re-purposes 
sensor-rich wristbands in retail to clinical, real-time monitoring of physical activity levels, posture detection and heart rate measurements.

Appelboom et al. 2014 provide review of sensors and their clinical applications discussed in literature that include cardiopulmonary and vascular monitoring, glucose home monitoring, neurological function monitoring, physical therapy and rehabilitation. The authors note that neurological function monitoring is one area, where neurological monitoring with smart wearable sensors (SWS) has great potential and success, especially in post-operative management, outpatient care and rehabilitation medicine (Appelboom et al. 2014). Those smart wearable sensors have the ability for accurate measurement of gait, limb paralysis, cerebral palsy with diagnostic capabilities of early detection of Parkinson's and Alzheimer's diseases.

One study determined that sensors are a reliable method to analyze gait in children with cerebral palsy (CP), while not all sensors could be treated as reliable and accurate (Kuo et al. 2009). Kirste et al. (2014) studied the use of accelerometer sensors in continuous monitoring of Alzheimer's patients and healthy control subjects and showed that with the novel algorithm the changes in everyday behavior are detectable in accelerometric behavior with the $91 \%$ accuracy even in absence of major clinical behavioral impairments. Use of inertial sensors with auditory and visual feedback also showed rehabilitative capabilities of sensors in patients with gait disturbances due to cerebral palsy (Baram and Lenger 2012). Appelboom et al. (2014) cite examples of various 
research that demonstrate clinical relevance and potential for clinical utility the integration of smart wearable sensors into healthcare to allow the medical professionals earlier detection of diseases, monitoring patient recovery and rehabilitation. The authors remark on the evolution of smart wearable sensors and their ability to monitor mobility, symptoms and other health indicators, which shows a great potential on transforming the healthcare system and patient behavior; continuous improvement of technologies and the need to integration and adoption of these technologies with infrastructure of evidence regarding reliability, validity and responsiveness of those applications across the healthcare field as well as collaboration between physicians, patients, engineers and establishment of guidelines for preventative and post-operative monitoring with the emphasis on physician-patient relationship (Appelboom et al. 2014). 


\section{CHAPTER 5. CASE APPLICATION}

\subsection{Introduction to the Problem}

According to StartUp Health, investment in digital health hopped $\$ 1.35$ billion in the first quarter of 2014 . Six themes (representing about $50 \%$ of last year's funding) emerged, including EHR/clinical workflow, analytics/big data, digital medical devices (clinically-oriented products with specific disease/condition focus), wearables/biosensing (consumer-oriented products with generalized biosensors), population health management, healthcare consumer engagement (purchasing of health insurance, healthcare services and products, intended for B2B and B2C markets) (Stoaks 2014).

Ash and Bates (2005) indicate that comprehensive national surveys with a high response rate are not available, and data in their study comes from the industry resources that may have some vested interests in IT usage or selection. The authors also indicate that small practices are less likely to adopt comparing to larger ones with various adoption gaps between the types of practices (pediatric, internal medicine etc.) Another interesting aspect provided by the authors is that there is a considerable amount of international experience (for example, Sweden, the Netherlands and Australia) that United States can gain insights from (Ash and Bates 2005).

In diffusion and adoption of health information technology, the physicians should consider factors that are environmental (financial and safety, social and 
behavioral), organizational, personal and technical (for example, ability of systems to interoperate with each other) in nature (Ash and Bates 2005).

One important study about the attitudes of physicians toward technology implementation was performed by Morton and Wiedenbeck using the framework grounded in Diffusion of Innovations Theory and TAM, while being conducted at the University of Mississippi Medical Center (UMMC) (Morton and Wiedenbeck 2009) The researchers acknowledged that their findings might not be generalized to other physician's offices, since the study was limited to one large healthcare system, however, they revealed an overwhelming need for customizable and flexible IT products (Morton and Wiedenbeck 2009).

In the era of rising healthcare costs, spinal problems are a burden to the society, since high costs are incurred as a result of spine health issues, including hospital stays, absence from work, disablement expenses, in addition to loss in overall quality of life (Farra et al. 2011). About $80 \%$ of adults will experience back pain at some point in life, some may need surgical intervention and roughly $10 \%$ of those will have a relapse in spine health condition (Farra et al. 2011). The Bureau of Labor Statistics reports that more than 130,000 lost-time cases of work-related back pain, sprains, stains and tears, carpal tunnel, tendonitis are associated with healthcare social assistants (Premier 2015). Americans spend at least $\$ 50$ billion each year on low back pain, the most common cause of jobrelated disability and a leading contributor to missed work. While most 
occurrences of low back pain resolve within days, some cases persist, resulting in chronic disability.

Historically, the surgical treatment of low back pain with spinal fusion has had relatively poor success with only $25 \%$ of patients reporting vast improvement following surgery. Because of this, health care insurers are demanding better methods for tracking surgical indications and outcomes. There are various indicators that could be tracked for back pain (Malliou 2006). The Oregon Health and Science University (OHSU) Multi-disciplinary Spine Clinic utilizes the Integrated Survey System (ISS, Dynamic Clinical Systems, Hanover, NH) to collect patient research data (e.g., SF-12, Oswestry Disability Index(ODI)) via a computer questionnaire (data is stored in a secured database).

\subsection{Problem Statement}

In June of 2007, OHSU started to use electronic database in order to track patients before their first visit and throughout their entire care at OHSU. Patients would fill out confidential surveys tracking factors like pain level, mobility, overall quality of life and level of disability. Since then the database has not been integrated well into physicians' routines so it could be impactful in physicianpatient relationships and communication levels. OHSU is looking to enhance the database through acquisitions of technologies that would correspond to the needs of physicians, analyze important patient characteristics, and give a useful snapshot of patients' issues that could lead to additional guidance and better treatment successes (Figure 10). 

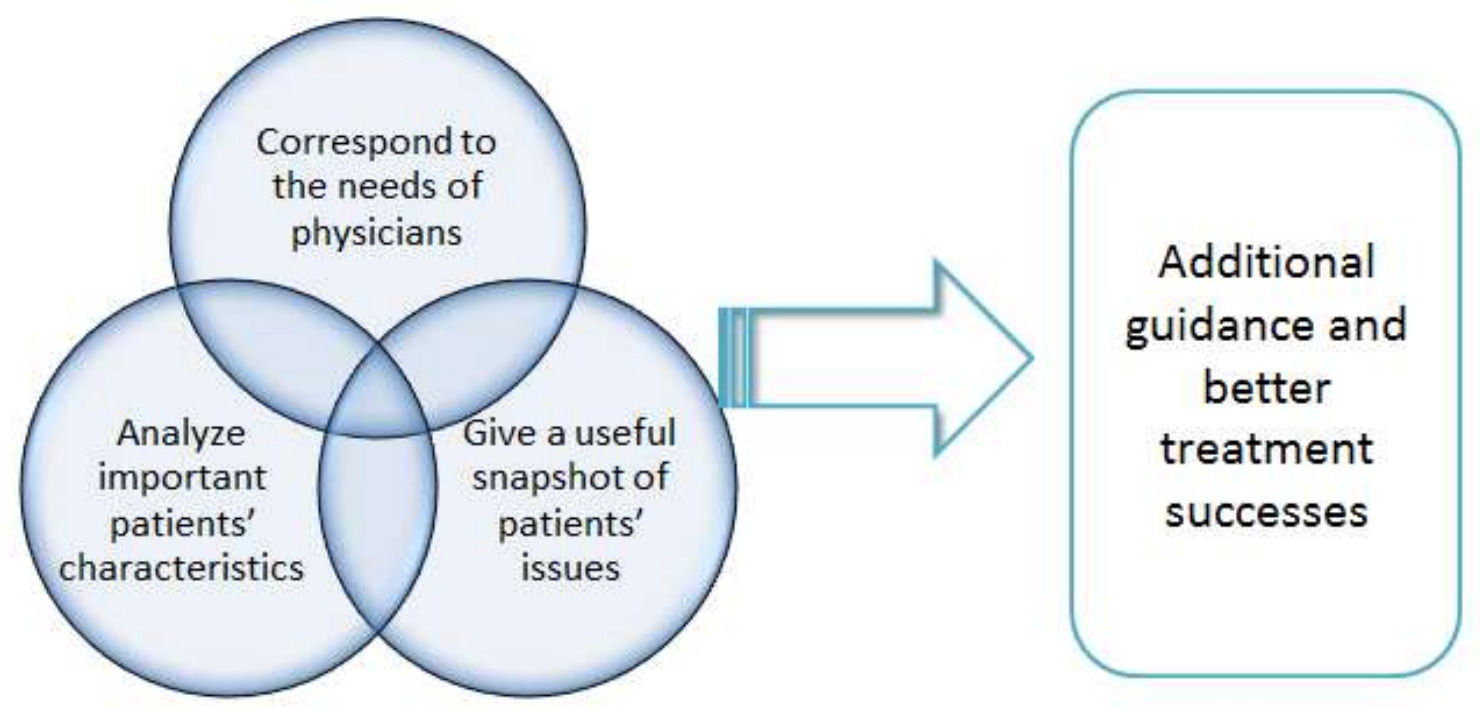

Figure 10 Main qualities of the technological system according to the needs physicians

The ISS system started at OHSU has roughly 14,000 patients with over 225,000 patient visits. The patient questionnaire takes roughly $8-10$ minutes to complete and can be accomplished on computer or paper. Roughly $20 \%$ of patients do not attempt the questionnaire reporting insufficient time as the number one reason. $25 \%$ percent of patients that start the questionnaire do not complete it. This high proportion of patients that fail to record their response results can be attributed to a database designed in the 1990's that does not take advantage of current graphic-user-interface (GUI) technology, mobile phone technology, has difficulty in quick data extraction, and finally, does not integrate with recently developed electronic health record (EHR) systems (Table 22). Finally, the system is onerous for surgeons to obtain data during patient clinical 
visits leaving these important patient measures out of point-of-care decision making.

Table 22 Pros and Cons of the current OHSU spine patient outcome database (Integrated Survey System (ISS, Dynamic Clinical Systems, Hanover, NH)).

\begin{tabular}{|c|c|}
\hline Pros: & Cons: \\
\hline $\begin{array}{l}\text { Validated research outcome } \\
\text { measures (e.g., SF-12, } \\
\text { Oswestry Disability Index (ODI) } \\
\text { (Niskanen 2002) }\end{array}$ & $\begin{array}{l}\text { Roughly } 20 \% \text { of patients to not attempt } \\
\text { questionnaire (June } 7 \text { - March } 2014 ; \\
847 / 5040((17 \%))\end{array}$ \\
\hline \multirow[t]{4}{*}{$\begin{array}{l}\text { Excellent patient reminder } \\
\text { system }\end{array}$} & $\begin{array}{l}75 \% \text { questionnaire completion rate (June } 7 \\
\text { - March } 2014 ; 3807 / 5040(75 \%) \text { ). Note: } \\
\text { insufficient time quoted as number one } \\
\text { reason for not completing (takes roughly } \\
20 \text { minutes to complete). }\end{array}$ \\
\hline & $\begin{array}{l}\text { No physician graphic user interface for } \\
\text { ease of data inquiry }\end{array}$ \\
\hline & $\begin{array}{l}\text { Labor intensive data input and data } \\
\text { extraction (e.g., paper questionnaires } \\
\text { manually inputted) }\end{array}$ \\
\hline & $\begin{array}{l}\text { Failure to integrate directly with OHSU } \\
\text { Electronic Health Record (Epic) }\end{array}$ \\
\hline
\end{tabular}

Oregon Health and Science University maintains the Functional Outcomes Program, which studies the treatment of chronic back pain and other conditions (Newman 2009; OHSU 2015). The goals of the program for patient care is targeting improvements in physical function; better pain management; daily activity management; preventative care aimed to decrease the need for future treatments. Wearable medical devices could enhance traditional patient/physical 
communication, improve patient adherence to medication regimens and potentially lead to better health outcomes (Slade Shantz and Veillette 2014).

Wearable sensor technologies could monitor patients' mobility, gait, tremor and other health indicators and daily activity in real time that could allow for simple, non-invasive, tracking of spine care that may lead to increased patient engagement, integration, feedback, post-surgery analysis, monitoring of patient's condition, patient's data extraction and analysis and possibly aiding in better diagnosis, intervention, adherence to treatment for the betterment of quality of care.

\subsection{Research Objective}

Wearable medical devices that track mobility of patients after spinal procedures could help surgeons in providing post-operative care, analysis of treatment outcomes and patient mobility. The assessment of those devices by physicians is a complex process associated with various perspectives and criteria.

Therefore, the objective of this research is to assess the potential for technology adoption of those wearable medical devices through development of a hierarchical decision-making model (HDM) that incorporates the relevant perspectives and criteria encompassing the needs of hospital neurological surgery and orthopedics department.

The proposed research builds on existing body of knowledge researched through literature review and background of the field and expand the health 
technology assessment field by implementation of holistic, comprehensive and multi-perspective approach to technology assessment in wearable sensor products adoption for pervasive care in neurosurgery and orthopedics.

Below is the short list of research procedures implemented for this study:

- Extensive literature review provides justification for the criteria used.

- Development of a hierarchical decision-making model (HDM) that incorporates important aspects of the needs of neurological surgery and orthopedics department with the possible alternative solutions.

- Validation of the model done by experts in the field (Qualtrics software used)

- Seven expert panels for quantification are established.

- Quatrics data in xml format is transferred with Ruby script into R CRAN AHP package module.

- Data provided by the experts' judgements is analyzed with $\mathrm{R}$ software and PCM software for validation purposes.

- HDM analysis is done with R and Excel. Discussion of priorities synthesis, sensitivity analysis and scenario analysis is performed.

- Analysis and interpretation of the data with the possibility of model generalization for possible hospital-wide and nation-wide acceptance.

The main study questions are: 
- What are the criteria for assessing technology adoption potential of the wearable sensor products for pervasive care in neurosurgery and orthopedics?

- What are the wearable sensor products that have potential for adoption for pervasive care in neurosurgery and orthopedics?

- Which health technology assessment perspectives have the highest importance from physician's perspective according to the experts?

- Which wearable sensor product has the highest adoption potential?

- How does change in values of perspectives impact the changes of rankings of wearable sensor products? 


\section{CHAPTER 6. CASE STUDY MODEL DEVELOPMENT}

\subsection{Research Phases}

The main research phases are shown in Figure 11 and described below:

Literature analysis and

HDM model development
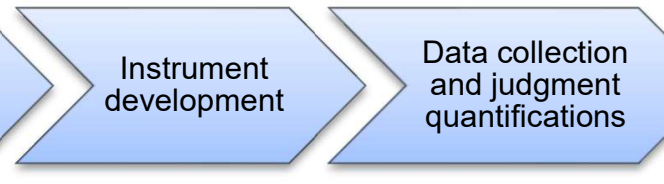

Data analysis, conclusions, recommendations

Figure 11 Major research phases

\subsubsection{Phase 1: Literature analysis and HDM model development}

During this phase the relevant literature in implementation factors, surveys, expert evaluations and other sources were studied to outline the implementation factors. The implementation factors were validated through the expert panels and reduced to a concise list that was tested with the questionnaire instrument.

\subsubsection{Phase 2: Instrument development}

Expert questionnaire instrument was developed for model validation and quantifications. The list of experts was constructed via snowball method. Personalized letter with request for participation in the study was sent to each one of them. In case some experts were not able to participate in the study, they were asked for a referral to a substitute expert with the required level of expertise 
in a particular field and specialization. Invitation letters were sent over email (Appendix A), followed by the links to the Qualtrics online site and consent letters were sent to the experts. Explanations of the model, perspectives, criteria and alternatives were provided with the research instrument in Qualtrics. Any questions were resolved over phone, email or face-to-face meeting with follow-up procedures for completion of the research instrument over email and phone.

\subsubsection{Phase 3: Data collection and judgment quantification}

This is a stage that included expert judgment quantifications for data collection purposes. Expert judgments were quantified across the levels of the decision hierarchy. The experts were asked to analyze the relative importance of the criteria with respect to the higher level criterion, for example: strategic goals with respect to the mission etc. Data collection was done through Qualtrics software. Data was saved in xml format for future analysis.

\subsubsection{Phase 4: Data analysis, conclusions, outlining recommendations}

Data analysis is done with Qualtrics, Ruby, R, PCM software and Excel. Once the data is analyzed, I outlined a set of recommendations, procedures and frameworks that could impact the direction of research in HIT management. Healthcare industry is very concerned about clinical benefits, however, the realization that those are difficult to achieve without good user experience, especially in hospital settings, is apparent. With mobile wearable technology overwhelming the market and becoming more common for everyday use, 
healthcare industry will be more involved, hoping for more involvement from the patients and better clinical results and use from the doctors.

\subsection{Model Development}

Model development in this research consists of two phases, which are the literature review and the expert evaluation. As a part of the model development phase, a preliminary assessment model has been constructed based on a comprehensive literature review. For content validity purposes, the preliminary model is subjected to the expert evaluation by capturing experts' judgment on suitability of the decision variables employed. Based on their comments and feedback, new variables may be added. Also some of the existing variables may be eliminated from the model. After expert evaluations are captured, the preliminary assessment model is revised and finalized. Due to the nature of the methodology (Hierarchical Decision Modeling) used in this research, research model and data collection instrument design are directly related. As a result, data collection instrument is revised based on the final model.

The development of the model components known as perspectives, criteria and alternatives has been done through a comprehensive literature review.

\subsubsection{Financial perspective}

Financial perspective is mentioned in the literature from rising costs of healthcare to high costs of systems implementation and management as well as 
loses from unsuccessful software applications. Some researchers indicated that facilitating conditions like financial rewards have been main factors to positively affect behavioral intention (Aggelidis and Chatzoglou 2009). The conclusions from study by one group of researchers stated that financial position indeed relates to HIT adoption in mid-term and long-term planning (Shen and Ginn 2012). Goldzweig et al. (2009) have noted that the costs still remain the numberone barrier cited by surveys assessing adoption, and stressed the need for a better alignment between "who pays" and "who benefits" from health IT. Implementation of financial rewards for quality improvement and for public reporting of quality performance measures can sometimes mitigate information systems issues and increase their use in the healthcare setting (Miller and Sim 2004).

Through my independent studies, besides the abovementioned articles, I have found a large number of researchers studying importance of financial incentives, identification of financial barriers and outlining financial attributes that are fundamental for healthcare IT implementation (Cresswell and Sheikh 2012; Ash and Bates 2005; Furukawa 2011; Leu et al. 2008; Blumenthal 2009; André et al. 2008; Goldberg 2012; Linder et al. 2007; Zandieh et al. 2008; Dixon et al. 2010; Jung 2006; Ortega Egea and Román González 2011; Im et al. 2008; Simon et al. 2007; Randeree 2007; Fonkych and Taylor 2005; McGinn et al. 2011; Martich and Cervenak 2007; Boonstra and Broekhuis 2010). 
In one study on defining and measuring successful emergency care networks, the researchers conclude that there are large gaps in understanding and measuring effects of those networks on outcomes, what disease conditions to target and how to best allocate resources (Glickman et al. 2010). Pilgrim et al. (2010) also note that there is significant fragmentation of the emergency care system with poor coordination, inefficient use of resources and therefore suboptimal patient outcomes. In a study of information technology implementation, the authors report various financial impacts (average monthly revenue increase due to better billing practices); clinical practice satisfaction as well as the support of the core mission of providing care (Goldzweig et al. 2009). Sayek (2013) also wrote on the importance of examining strength and weaknesses in the delivery of surgical care in the community and ability to formulate balanced policies for resource allocation and access to surgery.

\subsubsection{Technical perspective}

Technical aspects are very important to consider, but most importantly, to assess how well they will fit in within the multiple perspectives scenario, whether those technical capabilities would be a good fit and whether they get a good use under the current circumstances. Technical perspective is mentioned extensively in the literature (Moores 2012; Wu et al. 2007; Chen and Hsiao 2012; Liang et al. 2011; Cresswell and Sheikh 2012; Bodenheimer and Grumbach 2003; Kim and Chang 2006; Dünnebeil et al. 2012; Chen et al. 2010; Lorence and Churchill 2005; Zhang and Liu 2010; Tsiknakis et al. 2002; Vedvik et al. 2009; Bates et al. 
2003; Robert Wood Johnson Foundation 2010; Miller and Sim 2004; Angst et al. 2010; Handy et al. 2001; Greenhalgh et al. 2009; Menachemi and Brooks 2006; Boonstra and Broekhuis 2010; Rosemann et al. 2010; Tyler 2001; Rahimpour et al. 2008; Rind and Safran 1993; Bowens et al. 2010; Valdes et al. 2004; Jian et al. 2012; Blumenthal 2009; Yoon-Flannery et al. 2008; Ludwick and Doucette 2009; Ortega Egea and Román González 2011, Palacio et al. 2009; Goroll et al. 2008; Zhang and Liu 2010; Simon et al. 2007; Police et al. 2011; Glaser et al. 2008).

Pai and Huang (2011) presented a study of HIT adoption by district nurses, head directors and other related personnel, where TAM was used with external variables (information quality, service quality and system quality).

Perceived usefulness and perceived use are outlined to be the main two variables influencing attitude toward using the system. Just like perceived usefulness, the concept of ease of use has been known from Davis's Technology Acceptance model (Davis 1989) and it is the user's perception of the extent to which using a particular system would be free of effort (Davis 1985; Davis and Venkatesh 1996).

Aggelidis and Chatzoglou (2009) examined the use of health technology acceptance with the use of modified and extended TAM. Perceived ease of use and perceived usefulness were the most important factors of direct influence on behavioral intention. 
A large body of research has shown that perceived ease of use significantly impacts technology acceptance and influences user's decisionmaking process (Karahanna and Straub 1999; Cheng 2012; Kim and Chang 2006; Dixon 1999; Chow et al. 2012a; Chow et al. 2012b; Chen and Hsiao 2012; Vishwanath et al. 2009; King and He 2006; Legris et al. 2003; Ayatollahi et al. 2009; Cresswell and Sheikh 2012; Despont-Gros 2005; Davis and Venkatesh 1996; Yusof et al. 2008; Garcia-Smith and Effken 2013; Melas et al. 2011; Liu and Ma 2005; Dünnebeil et al. 2012; Jian et al. 2012; Carayon et al. 2011 and others).

The system should have the ease of use in aspects of data extraction, access and storage. The health technology system could be packed with valuable data, but if it is not easy for the user to access it (in a timely manner with not a significant amount of effort), the value of that system to the user diminishes greatly. Easy access to information facilitates communication and decisionmaking in healthcare (Kim and Chang 2006). The concept of accessibility, data extraction and storage, is studied in the context of healthcare management, technology acceptance and software or application selection (Roth et al. 2009; Mäenpää et al. 2009; Chumbler et al. 2011; Zhang and Liu 2010; Rind and Safran 1993; Ayatollahi et al. 2009; Garcia-Smith and Effken 2013; Millstein and Darling 2010; Furukawa 2011; Leu et al. 2008; Dünnebeil et al. 2012; Alper and Olson 2010; Mäenpää et al. 2009; Ludwick and Doucette 2009; Zhang and Liu 
2010; Vedvik et al. 2009; Sanchez et al. 2013; Wu et al. 2009; Chen et al. 2010; Goldberg 2012).

System's user should also be able to search the system in a timely effortless manner with acceptable and meaningful results. Having a good quality search engine with quick searching capabilities could greatly benefit a small practice; however, some physicians may not feel like they need an elaborate searching system and may opt out for software with the modest acceptable searching capabilities. Researchers have noted the feature of good data mining or data search (Ayatollahi et al. 2009; Alper and Olson 2010; Randeree 2007; Palacio et al. 2009).

Perceived usefulness is "the degree to which individual believes that using a particular system would enhance his or her job performance" (Davis 1985; Davis and Venkatesh 1996). This criteria has its roots in Technology Acceptance Model (Davis 1989), identifies the user's perception of the degree to which using a particular system will improve his or her performance. The psychological origins of the concept are grounded in social presence theory, social influence theory and Triandis modifications to the theory of reasoned action (Karahanna and Straub 1999). Perceived usefulness has been shown to have a great impact on technology acceptance in healthcare (Cheng 2012; Kim and Chang 2006; Chen and Hsiao 2012; King and He 2006; Cresswell and Sheikh 2012; DespontGros et al. 2005; Yusof et al. 2008; Melas et al. 2011; McGinn et al. 2011; Morton and Wiedenbeck 2009). The concepts of TAM and relative research has been 
instrumental in explaining how beliefs about systems lead users to have positive attitudes toward systems; intentions to use these systems and system use (Karahanna and Straub 1999). Perceived usefulness will be rated in terms of enhancing job performance.

The system should be able to function well with other applications in the network, local and shared. Alper and Olson (2010) note that interoperability is important to improve and coordinate care delivery. While in the United States most patients receive care from several providers, a lack of interoperability in the network would mean that physicians do not have access to a complete record for a patient and a "master record" might not exist or might not be complete at any point in time (Alper and Olson 2010). Different systems will provide various levels of interoperability and the users may require more or less advanced systems for their clinics. A number of researchers stressed the importance of interoperability of the information technology system as expressed by administrators, physicians and other technology users and the need to invest in improvements in it (Cresswell and Sheikh 2012; Alper and Olson 2010; Ash and Bates 2005; Furukawa 2011; Goldzweig et al. 2009; Dünnebeil et al. 2012; Jian et al. 2012; Glaser et al. 2008; Yao and Kumar 2013; Blumenthal 2009; Yoon-Flannery et al. 2008; Degoulet et al. 1995; Mäenpää et al. 2009; Dixon et al. 2010; Zaroukian 2006; Jung 2006; Lapinsky et al. 2008; Palacio et al. 2009; Goroll et al. 2008; Bufalino et al. 2011; Blumenthal 2010; DePhillips 2007; Box et al. 2010; Kazley 
and Ozcan 2008; Fonkych and Taylor 2005; McGinn et al. 2011; Zhang and Liu 2010; Tsiknakis et al. 2002).

\subsubsection{Organizational perspective}

In addition to the technical and financial aspects of wearable technology adoption, it is also important to consider organizational aspect that plays a crucial role in adoption process. Box et al. (2010) state that throughout health information technology implementation, success requires a careful balance of technical, clinical and organizational factors. Cresswell and Sheikh (2012) dedicate an empirical and interpretative review study on organizational issues in HIT adoption and implementation.

Organizational issues were described by the number of researchers: (Morton and Wiedenbeck 2009; McGinn et al. 2011; Boonstra and Broekhuis 2010; Yi et al. 2006; Kukafka et al. 2003; Moores 2012; Weiner et al. 2011; Davis 1989; Yarbrough and Smith 2007; Lanham et al. 2012; Brand et al. 2005; Chen et al. 2010; Johnson et al. 2012; Goldberg 2012; Burton-Jones and Hubona 2006; Pynoo et al. 2011; Alper and Olson 2010; Chumbler et al. 2011; Zaroukian 2006; Kim and Chang 2006; Ash and Bates 2005).

With any new system, there will be some time for adjustment from an organizational point of view and some training required. Some systems may require more or less training, and physicians need to be aware of those variables. In addition to the possible financial impact the process of training will require, it may also involve hiring more personnel or using vendors' training 
human resources. The intensity, timing and availability of training and support post-implementation affect user experience (Ludwick and Doucette 2009). The issue of training is an important one to consider and has been mentioned by various researchers (Lee and Xia 2011; Noblin et al. 2013; Ludwick and Doucette 2009; Yeager et al. 2010; Pilouras et al. 2011; Morton and Wiedenbeck 2009; Ayatollahi et al. 2009; McGinn et al. 2011; Police et al. 2011; Yi et al. 2006; Chaudhry et al. 2006; Moores 2012; Kumar and Aldrich 2010).

The availability of tech support is important in software selection, with some that may have straightforward, personalized system, or online-only system, or the vendor might not provide tech support. Depending on the IT infrastructure and the in-house capabilities, physicians need to carefully examine this aspect to decide how important tech support is for them and how much tech support they will require. Tech support, or lack of thereof, is an issue described by researchers, with bright examples in qualitative studies (Boonstra and Broekhuis 2010; Miller and Sim 2004; Holden and Karsh 2010; Pynoo et al. 2011; Wu et al. 2007; Yu et al. 2009; Valdes et al. 2004; Goroll et al. 2008; Lustria et al. 2011).

\subsubsection{Patient Perspective}

Patient experience refers to physician's perspective of patient experience with the system (Garcia-Smith and Effken 2013) and is described in the Clinical Information Systems Success Model and IT End-User Satisfaction Model (Mahmood et al. 2000). After an extensive literature review, the researchers reveal that IT end-user satisfaction factors fall into three major categories: 
perceived benefits and convenience (for example, perceived ease of use and usefulness), user background and involvement (user experience falls in this category) and organizational attitude and support (Mahmood et al. 2000). User experience and satisfaction was also outlined in the many studies (Wolf et al. 2014; Rose et al. 2005; Im et al. 2008; Moores 2012; Haron et al. 2012; Ludwick and Doucette 2009; Venkatesh and Davis 2000; Chiasson et al. 2007; Premkumar and Bhattacherjee 2008).

Clinical benefits, sometimes called patient-related benefits of HIT (Police et al. 2011), is a very important and measurable factor in healthcare. Clinical benefits measures give opportunity to physicians to track, to report and review their notes, procedures, routines and diagnoses. Miller and Sim (2004) commented on the dependence of quality benefits on the amount of viewable clinical data. Shields et al. (2007) also emphasize on the importance of access to clinical benefits associated with HIT.

Privacy concerns have been some of the well noted issues for physicians while choosing a software system. Issues of privacy are mentioned in numerous research articles (Randeree 2007; Simon et al. 2007; Bates et al. 2003; Bufalino et al. 2011; Handy et al. 2001; Lorenzi et al. 2009; Angst et al. 2010; Glaser et al. 2008; Palacio et al. 2009; Kazley and Ozcan 2007; Lustria et al. 2011; Morton and Wiedenbeck 2010; Tyler 2001; DePhillips 2007; Blumenthal 2010; YoonFlannery et al. 2008; Goroll et al. 2008; Ash and Bates 2005; Zheng et al. 2010). The concept of data security has been brought up by many researchers as well 
as the government (Chen et al. 2010; Lorence and Churchill 2005; Zhang and Liu 2010; Tsiknakis et al. 2002; Vedvik et al. 2009; Rind and Safran 1993; Alper and Olson 2010; Bowens et al. 2010; Yusof et al. 2008; Liu and Ma 2005; Dünnebeil et al. 2012). The concept of data security, encryption and secure storage has been described in the literature review sections above. Differences of in-cloud vs. remote storage have been discussed as having various security features.

\subsubsection{Interpersonal perspective}

The importance of various relationships in people lives and workplaces can impact their perceptions, assessment and adoption. Perceived impact of dynamics of the relationship, whether it is doctor-doctor, doctor-nurse and doctorpatient should not be overlooked.

Interpersonal perspective has some elements of social, organizational and personal dynamics (Cresswell and Sheikh 2012). The importance of sharing and communication among various levels in the organization and outside (doctorpatient) and the ability of the software to provide that capability and perhaps improve the communication and important flow of information should be considered in technology adoption process. Interpersonal issues have been discussed in the research literature (Beckett et al. 2011; Wu et al. 2007; Yu and Gagnon 2009; Yarbrough and Smith 2007; Chen and Hsiao 2012; Frambach and Schillewaert 2002; Cheng 2012; Yang 2004; Dünnebeil et al. 2012; Liu and Ma 2005; Yusof et al. 2008; Chiasson et al. 2007; Makam et al. 2014). Kumar and Aldrich (2010) performed a SWOT analysis of a nationwide HIT system 
implementation in US, and in the section of "threats" included statements that greater standardization could remove the "human touch" between healthcare practitioners and patients and the doctor-patient relationship might turn into a new triad, where healthcare technology could be acting as a proxy for all who provide patient with care.

\subsubsection{Taxonomy of the main model perspectives}

Taxonomy of perspectives is shown in Table 23. 
Table 23 Taxonomy of the main perspectives used in the model.

\begin{tabular}{|c|c|}
\hline Perspectives & References \\
\hline Financial & $\begin{array}{l}\text { Aggelidis and Chatzoglou 2009; Shen and Ginn 2012; Goldzweig et } \\
\text { al. 2009; Cresswell and Sheikh 2012; Ash and Bates 2005; } \\
\text { Furukawa 2011; Leu et al. 2008; Blumenthal 2009; André et al. 2008; } \\
\text { Goldberg 2012; Linder et al. 2007; Zandieh et al. 2008; Dixon et al. } \\
\text { 2010; Jung 2006; Ortega Egea and Román González 2011; Im et al. } \\
\text { 2008; Simon et al. 2007; Randeree 2007; Fonkych and Taylor 2005; } \\
\text { McGinn et al. 2011; Martich and Cervenak 2007; Boonstra and } \\
\text { Broekhuis 2010; Tsiknakis et al. 2002; Glickman et al. 2010; Pilgrim } \\
\text { et al. 2010; Sayek 2013; DeLone and McLean } 2003\end{array}$ \\
\hline Technical & $\begin{array}{l}\text { Moores 2012; Wu et al. 2007; Chen and Hsiao 2012; Liang et al. } \\
\text { 2011; Cresswell and Sheikh 2012; Bodenheimer and Grumbach } \\
\text { 2003; Kim and Chang 2006; DeLone and McLean 2003; Pai and } \\
\text { Huang 2011; Davis 1985; Davis and Venkatesh 1996; Aggelidis and } \\
\text { Chatzoglou 2009; Karahanna and Straub 1999; Cheng 2012; Kim } \\
\text { and Chang 2006; Dixon 1999; Chow et al. 2012a; Vishwanath et al. } \\
\text { 2009; King and He 2006; Legris et al. 2003; Ayatollahi et al. 2009; } \\
\text { Cresswell and Sheikh 2012; Despont-Gros 2005; Davis and } \\
\text { Venkatesh 1996; Yusof et al. 2008; Garcia-Smith and Effken 2013; } \\
\text { Melas et al. 2011; Liu and Ma 2005; Dünnebeil et al. 2012; Jian et al. } \\
\text { 2012; Carayon et al. 2011; Roth et al. 2009; Mäenpää et al. 2009; } \\
\text { Chumbler et al. 2011; Zhang and Liu 2010; Rind and Safran 1993; } \\
\text { Ayatollahi et al. 2009; Garcia-Smith and Effken 2013; Millstein and } \\
\text { Darling 2010; Furukawa 2011; Leu et al. 2008; Dünnebeil et al. 2012; } \\
\text { Alper and Olson 2010; Mäenpää et al. 2009; Ludwick and Doucette } \\
\text { 2009; Vedvik et al. 2009; Sanchez et al. 2013; Wu et al. 2009; Chen } \\
\text { et al. 2010; Goldberg 2012; Karahanna and Straub 1999; Cheng } \\
\text { 2012; Kim and Chang 2006; Chen and Hsiao 2012; King and He } \\
\text { 2006; Cresswell and Sheikh 2012; Despont-Gros et al. 2005; Yusof } \\
\text { et al. 2008; Melas et al. 2011; McGinn et al. 2011; Morton and } \\
\text { Wiedenbeck 2009; Ash and Bates 2005; Goldzweig et al. 2009; Jian } \\
\text { et al. 2012; Glaser et al. 2008; Yao and Kumar 2013; Blumenthal } \\
\text { 2009; Yoon-Flannery et al. 2008; Degoulet et al. 1995; Mäenpää et } \\
\text { al. 2009; Dixon et al. 2010; Zaroukian 2006; Jung 2006; Lapinsky et } \\
\text { al. 2008; Palacio et al. 2009; Goroll et al. 2008; Bufalino et al. 2011; } \\
\text { Blumenthal 2010; DePhillips 2007; Box et al. 2010; Kazley and } \\
\text { Ozcan 2008; Fonkych and Taylor 2005; McGinn et al. 2011; Zhang } \\
\text { and Liu 2010; Tsiknakis et al. 2002. }\end{array}$ \\
\hline
\end{tabular}




\begin{tabular}{|c|c|}
\hline Perspectives & References \\
\hline Organizational & $\begin{array}{l}\text { Box et al. 2010; Cresswell and Sheikh 2012; Ludwick and Doucette } \\
\text { 2009; Lee and Xia 2011; Noblin et al. 2013; Ludwick and Doucette } \\
\text { 2009; Yeager et al. 2010; Pilouras et al. 2011; Morton and } \\
\text { Wiedenbeck 2009; Ayatollahi et al. 2009; McGinn et al. 2011; Police } \\
\text { et al. 2011; Yi et al. 2006; Chaudhry et al. 2006; Moores 2012; } \\
\text { Kumar and Aldrich 2010; Moores 2012; Helfrich et al. 2007; Yi et al. } \\
\text { 2006; Kukafka et al. 2003; Zaroukian 2006; Aggelidis and } \\
\text { Chatzoglou 2009; Holden and Karsh 2010; Goroll et al. 2008; Pynoo } \\
\text { et al. 2011; Wu et al. 2007; Randeree 2007; Hung et al. 2012; } \\
\text { Staples et al. 2002; Alhateeb et al. 2009; Chow et al. 2012b; Shibl et } \\
\text { al. 2013; Dillon and Morris 1996; Lanham et al. 2012; Cresswell and } \\
\text { Sheikh 2012; Kumar and Aldrich 2010; Dünnebeil et al. 2012; Li et al. } \\
\text { 1998; Ludwick and Doucette 2009; Moores 2012; Helfrich et al. 2007; } \\
\text { Yi et al. 2006; Kukafka et al. 2003; Zaroukian 2006; Aggelidis and } \\
\text { Chatzoglou 2009; Holden and Karsh 2010; Goroll et al. 2008; Pynoo } \\
\text { et al. 2011; Wu et al. 2007; Randeree 2007; Hung et al. 2012; } \\
\text { Staples et al. 2002; Alhateeb et al. 2009;; Moores 2012; Helfrich et } \\
\text { al. 2007; Yi et al. 2006; Kukafka et al. 2003; Zaroukian 2006; } \\
\text { Aggelidis and Chatzoglou 2009; Holden and Karsh 2010; Goroll et al. } \\
\text { 2008; Pynoo et al. 2011; Wu et al. 2007; Randeree 2007; Hung et al. } \\
\text { 2012; Staples et al. 2002; Alhateeb et al. 2009; Boonstra and } \\
\text { Broekhuis 2010; Miller and Sim 2004; Holden and Karsh 2010; } \\
\text { Pynoo et al. 2011; Wu et al. 2007; Yu et al. 2009; Valdes et al. 2004; } \\
\text { Goroll et al. 2008; Lustria et al. 2011 }\end{array}$ \\
\hline Patient & $\begin{array}{l}\text { Garcia-Smith and Effken 2013; Mahmood et al. 2000; Mahmood et } \\
\text { al. 2000; Rose et al. 2005; Im et al. 2008; Moores 2012; Ludwick and } \\
\text { Doucette 2009; Venkatesh and Davis 2000; Chiasson et al. 2007; } \\
\text { Premkumar and Bhattacherjee 2008; Police et al. 2011; Miller and } \\
\text { Sim 2004; Shields et al. 2007; Randeree 2007; Simon et al. 2007; } \\
\text { Bates et al. 2003; Bufalino et al. 2011; Handy et al. 2001; Lorenzi et } \\
\text { al. 2009; Angst et al. 2010; Glaser et al. 2008; Palacio et al. 2009; } \\
\text { Kazley and Ozcan 2007; Lustria et al. 2011; Morton and Wiedenbeck } \\
\text { 2010; Tyler 2001; DePhillips 2007; Blumenthal 2010; Yoon-Flannery } \\
\text { et al. 2008; Goroll et al. 2008; Ash and Bates 2005; Zheng et al. } \\
\text { 2010; Chen et al 2010; Lorence and Churchill 2005; Zhang and Liu } \\
\text { 2010; Tsiknakis et al. 2002; Vedvik et al. 2009; Rind and Safran } \\
\text { 1993; Alper and Olson 2010; Bowens et al. 2010; Yusof et al. 2008; } \\
\text { Liu and Ma 2005; Dünnebeil et al. 2012 }\end{array}$ \\
\hline Interpersonal & $\begin{array}{l}\text { Cresswell and Sheikh 2012; Beckett et al. 2011; Wu et al. 2007; Yu } \\
\text { and Gagnon 2009; Yarbrough and Smith 2007; Chen and Hsiao } \\
\text { 2012; Frambach and Schillewaert 2002; Cheng 2012; Yang 2004; } \\
\text { Dünnebeil et al. 2012; Liu and Ma 2005; Yusof et al. 2008; Chiasson } \\
\text { et al. 2007; Kumar and Aldrich 2010; Cresswell and Sheikh 2012; } \\
\text { Beckett et al. 2011; Wu et al. 2007; Yu and Gagnon 2009; Yarbrough } \\
\text { and Smith 2007; Chen and Hsiao 2012; Frambach and Schillewaert } \\
2002\end{array}$ \\
\hline
\end{tabular}




\subsubsection{Taxonomies of model criteria}

Taxonomies of model criteria with their descriptions and definitions for the model are presented in tables below (Table 24, Table 25, Table 26, Table 27, Table 28).

Table 24 Taxonomy of model criteria of Financial perspective

\begin{tabular}{|c|c|c|c|}
\hline Criteria & Descriptions & Definitions for model & References \\
\hline $\begin{array}{l}\text { Cost of } \\
\text { acquisition }\end{array}$ & $\begin{array}{l}\text { Cost is inclusive of the } \\
\text { product cost as well as } \\
\text { the cost involved in } \\
\text { research, marketing, } \\
\text { and accessibility costs }\end{array}$ & $\begin{array}{l}\text { Expenses } \\
\text { associated with the } \\
\text { purchase of the } \\
\text { wearable sensor } \\
\text { technology solution }\end{array}$ & $\begin{array}{l}\text { (Zaroukian } \\
\text { 2006; Girosi et } \\
\text { al. 2005) }\end{array}$ \\
\hline $\begin{array}{l}\text { Savings due } \\
\text { to economies } \\
\text { of scale }\end{array}$ & $\begin{array}{l}\text { The cost advantages } \\
\text { that enterprises obtain } \\
\text { due to size, output, } \\
\text { or scale of operation, } \\
\text { with cost per unit of } \\
\text { output generally } \\
\text { decreasing with } \\
\text { increasing scale as } \\
\text { fixed costs are spread } \\
\text { out over more units of } \\
\text { output }\end{array}$ & $\begin{array}{l}\text { Savings to the } \\
\text { department and } \\
\text { clinic due to } \\
\text { obtaining large } \\
\text { amounts if a } \\
\text { particular kind of } \\
\text { wearable solution for } \\
\text { their patients } \\
\text { (provided by the } \\
\text { suppliers and by the } \\
\text { larger pool of } \\
\text { patients served by a } \\
\text { wearable medical } \\
\text { device. }\end{array}$ & $\begin{array}{l}\text { (Walter 2008; } \\
\text { Fonkych and } \\
\text { Taylor 2005, } \\
\text { Viswanathan } \\
\text { 2005; Simon et } \\
\text { al. 2008; } \\
\text { Menachemi et } \\
\text { al. 2008) }\end{array}$ \\
\hline $\begin{array}{l}\text { Cost of } \\
\text { maintenance }\end{array}$ & $\begin{array}{l}\text { Costs associated with } \\
\text { maintenance of the } \\
\text { system }\end{array}$ & $\begin{array}{l}\text { Costs associated } \\
\text { with maintenance of } \\
\text { the system of the } \\
\text { wearable product. }\end{array}$ & $\begin{array}{l}\text { (Girosi et al. } \\
\text { 2005; Basoglu } \\
\text { et al. 2012) }\end{array}$ \\
\hline $\begin{array}{l}\text { Decrease in } \\
\text { hospital/clinic } \\
\text { costs }\end{array}$ & $\begin{array}{l}\text { Decrease in hospital } \\
\text { costs due to savings to } \\
\text { the department } \\
\text { associated with the } \\
\text { acquisition of the } \\
\text { technology solutions }\end{array}$ & $\begin{array}{l}\text { Decrease in overall } \\
\text { expenses incurred } \\
\text { by hospital in } \\
\text { providing services } \\
\text { due to the } \\
\text { technological } \\
\text { acquisition. }\end{array}$ & $\begin{array}{l}\text { (Bowens et al. } \\
\text { 2010; Shen and } \\
\text { Ginn 2012) }\end{array}$ \\
\hline
\end{tabular}


Table 25 Taxonomy of model criteria of Technical perspective

\begin{tabular}{|c|c|c|c|}
\hline Criteria & Descriptions & Definitions for model & References \\
\hline Reliability & $\begin{array}{l}\text { The quality or } \\
\text { state of being } \\
\text { reliable; an } \\
\text { attribute of a } \\
\text { system that } \\
\text { produces the } \\
\text { same results, } \\
\text { preferably } \\
\text { meeting or } \\
\text { exceeding } \\
\text { expectations }\end{array}$ & $\begin{array}{l}\text { A characteristic of a } \\
\text { wearable sensor } \\
\text { technological solution } \\
\text { providing accurate } \\
\text { measurements and } \\
\text { analytic reports; } \\
\text { consistent and stable } \\
\text { performance, free of } \\
\text { technical issues and } \\
\text { problems. }\end{array}$ & $\begin{array}{l}\text { (Box et al. 2010; } \\
\text { Banerjee et al. } \\
\text { 2012; Banos et } \\
\text { al. 2014; } \\
\text { Mäenpää et al. } \\
\text { 2009; Van Schaik } \\
\text { et al. 2004) }\end{array}$ \\
\hline $\begin{array}{l}\text { Physician's } \\
\text { ease of use }\end{array}$ & $\begin{array}{l}\text { The degree to } \\
\text { which an } \\
\text { individual } \\
\text { believes that } \\
\text { using a particular } \\
\text { system would be } \\
\text { free of physical } \\
\text { and mental effort. }\end{array}$ & $\begin{array}{l}\text { Physician's intuitive } \\
\text { and effortless } \\
\text { interaction with the } \\
\text { wearable sensor } \\
\text { technological solution } \\
\text { including the } \\
\text { accompanied software, } \\
\text { databases and } \\
\text { analytics/aggregate } \\
\text { reports. }\end{array}$ & $\begin{array}{l}\text { (Davis 1985; } \\
\text { Davis and } \\
\text { Venkatesh 1996; } \\
\text { Cheng 2012; Kim } \\
\text { and Chang 2006; } \\
\text { Chow et al. } \\
\text { 2012a; Chow et } \\
\text { al. 2012b; Chen } \\
\text { and Hsiao 2012) }\end{array}$ \\
\hline $\begin{array}{l}\text { Productivity } \\
\text { impact } \\
\text { (usefulness) }\end{array}$ & $\begin{array}{l}\text { The degree to } \\
\text { which individual } \\
\text { believes that } \\
\text { using a particular } \\
\text { system would } \\
\text { enhance his or } \\
\text { her job } \\
\text { performance }\end{array}$ & $\begin{array}{l}\text { The ability of the } \\
\text { complete technological } \\
\text { solution to enhance the } \\
\text { physician's job } \\
\text { performance (enabling } \\
\text { to provide better care, } \\
\text { diagnoses, etc.) }\end{array}$ & $\begin{array}{l}\text { (Davis 1985; } \\
\text { Davis and } \\
\text { Venkatesh 1996; } \\
\text { Cheng 2012; Kim } \\
\text { and Chang 2006; } \\
\text { Chen and Hsiao } \\
\text { 2012; King and } \\
\text { He 2006; } \\
\text { Cresswell and } \\
\text { Sheikh 2012). }\end{array}$ \\
\hline
\end{tabular}




\begin{tabular}{|c|c|c|c|}
\hline Criteria & Descriptions & Definitions for model & References \\
\hline Interoperability & $\begin{array}{l}\text { Ability of a } \\
\text { system to } \\
\text { exchange and } \\
\text { make use of } \\
\text { information; the } \\
\text { ability of different } \\
\text { information } \\
\text { technology } \\
\text { systems and } \\
\text { software } \\
\text { applications to } \\
\text { communicate, } \\
\text { exchange data, } \\
\text { and use the } \\
\text { information that } \\
\text { has been } \\
\text { exchanged. }\end{array}$ & $\begin{array}{l}\text { Vendors support of } \\
\text { consensus standards } \\
\text { that enables } \\
\text { communication and } \\
\text { data exchange of } \\
\text { wearable sensor } \\
\text { technology solution } \\
\text { with clinical software } \\
\text { applications and } \\
\text { databases. }\end{array}$ & $\begin{array}{l}\text { (Alper and Olson } \\
\text { 2010; Cresswell } \\
\text { and Sheikh 2012; } \\
\text { Ash and Bates } \\
\text { 2005; Furukawa } \\
\text { 2011; Goldzweig } \\
\text { et al. 2009; } \\
\text { Dünnebeil et al. } \\
\text { 2012; Jian et al. } \\
\text { 2012; Glaser et } \\
\text { al. 2008). }\end{array}$ \\
\hline $\begin{array}{l}\text { Ease of data } \\
\text { access }\end{array}$ & $\begin{array}{l}\text { The quality of } \\
\text { being available } \\
\text { when needed; } \\
\text { effective } \\
\text { coverage of the } \\
\text { data provided } \\
\text { throughout the } \\
\text { department. }\end{array}$ & $\begin{array}{l}\text { Ability of the wearable } \\
\text { sensor solution to } \\
\text { provide easy access to } \\
\text { data reports and } \\
\text { analytics. }\end{array}$ & $\begin{array}{l}\text { (Teng et al. 2008; } \\
\text { Cho et al. 2010; } \\
\text { Pilgrim et al. } \\
\text { 2010; Banos et } \\
\text { al. 2014; } \\
\text { Mäenpää et al. } \\
\text { 2009) }\end{array}$ \\
\hline
\end{tabular}


Table 26 Taxonomy of model criteria of Organizational perspective

\begin{tabular}{|c|c|c|c|}
\hline Criteria & Descriptions & Definitions for model & References \\
\hline $\begin{array}{l}\text { Training } \\
\text { Needed }\end{array}$ & $\begin{array}{l}\text { Organized activit } \\
\text { y aimed at } \\
\text { imparting } \\
\text { information and } \\
\text { /or instructions to } \\
\text { improve the } \\
\text { recipient's perfor } \\
\text { mance or to help } \\
\text { him or her attain } \\
\text { a required level } \\
\text { of knowledge or } \\
\text { skill. }\end{array}$ & $\begin{array}{l}\text { Organized activity } \\
\text { aimed at imparting } \\
\text { information and/or } \\
\text { instructions to help } \\
\text { medical staff attain a } \\
\text { required level of } \\
\text { knowledge or skill } \\
\text { related to wearable } \\
\text { product solution. }\end{array}$ & $\begin{array}{l}\text { (Ludwick and } \\
\text { Doucette 2009; Lee } \\
\text { and Xia 2011; Noblin } \\
\text { et al. 2013; Yeager et } \\
\text { al. 2010; Pilouras et } \\
\text { al. 2011; Morton and } \\
\text { Wiedenbeck 2009; } \\
\text { Ayatollahi et al. 2009; } \\
\text { McGinn et al. 2011; } \\
\text { Police et al. } 2011\end{array}$ \\
\hline $\begin{array}{l}\text { Tech } \\
\text { Support }\end{array}$ & $\begin{array}{l}\text { A service } \\
\text { provided by a } \\
\text { hardware or } \\
\text { software } \\
\text { company in the } \\
\text { form of help and } \\
\text { advice about } \\
\text { their products. }\end{array}$ & $\begin{array}{l}\text { A service provided by } \\
\text { a hardware or } \\
\text { software company } \\
\text { that supplies medical } \\
\text { staff with help and } \\
\text { advice about the } \\
\text { wearable product } \\
\text { solution. }\end{array}$ & $\begin{array}{l}\text { (Pilgrim et al. 2010; } \\
\text { Walker et al. 2008; } \\
\text { Zhou et al. 2015; } \\
\text { Lustria et al. 2011; } \\
\text { Sandberg et al. 2003) }\end{array}$ \\
\hline $\begin{array}{l}\text { Data } \\
\text { Support }\end{array}$ & $\begin{array}{l}\text { Ensuring data } \\
\text { continuity, } \\
\text { backup and } \\
\text { integration into } \\
\text { clinical practice. }\end{array}$ & $\begin{array}{l}\text { Support with overall } \\
\text { data integration: } \\
\text { formats, standards, } \\
\text { changes, downloads, } \\
\text { updates, etc. }\end{array}$ & $\begin{array}{l}\text { (Pilouras et al. 2011; } \\
\text { Degoulet et al. 1995; } \\
\text { Geum et al. 2011; } \\
\text { Paré and Sicotte } \\
\text { 2001; Bentley et al. } \\
\text { 2007; Cresswell and } \\
\text { Sheikh 2012; } \\
\text { Mendonça et al. 2004; } \\
\text { Khoumbati et al. } \\
\text { 2006; Murff et al. } \\
\text { 2003) }\end{array}$ \\
\hline $\begin{array}{l}\text { Competitive } \\
\text { Advantage }\end{array}$ & $\begin{array}{l}\text { Competitiveness } \\
\text { and advantage } \\
\text { resulting from } \\
\text { technological } \\
\text { innovations, } \\
\text { critical } \\
\text { technological } \\
\text { areas }\end{array}$ & $\begin{array}{l}\text { An advantage over } \\
\text { competitors gained } \\
\text { by clinics offering } \\
\text { patients greater } \\
\text { value by providing } \\
\text { greater benefits and } \\
\text { services as a result } \\
\text { of the wearable } \\
\text { sensor product } \\
\text { acquisition. }\end{array}$ & $\begin{array}{l}\text { (Azzone and Manzini } \\
\text { 2008; Wu et al. 2009; } \\
\text { Wang and Hsieh } \\
\text { 2014; Tran and Daim } \\
\text { 2008; Liyanage 1995; } \\
\text { Köhler and Som 2013; } \\
\text { Fonkych and Taylor } \\
\text { 2005; Jolly 2008) }\end{array}$ \\
\hline
\end{tabular}


Table 27 Taxonomy of model criteria of Patient perspective

\begin{tabular}{|c|c|c|c|}
\hline Criteria & Descriptions & $\begin{array}{l}\text { Definitions for } \\
\text { model }\end{array}$ & References \\
\hline $\begin{array}{l}\text { Patient } \\
\text { Experience }\end{array}$ & $\begin{array}{l}\text { The sum of all interactions, } \\
\text { shaped by an organization's } \\
\text { culture, that influence patient } \\
\text { perceptions across the } \\
\text { continuum of care ( The } \\
\text { Beryl Institute); aligned with } \\
\text { patient-centered care } \\
\text { principles }\end{array}$ & $\begin{array}{l}\text { Overall } \\
\text { patient's } \\
\text { satisfaction } \\
\text { with using the } \\
\text { wearable } \\
\text { product } \\
\text { solution. }\end{array}$ & $\begin{array}{l}\text { (Bächlin et al. } \\
\text { 2010; Morton } \\
\text { and Wiedenbeck } \\
\text { 2009; DeLia et } \\
\text { al. 2004; } \\
\text { Johnson et al. } \\
\text { 2012; Chen et al. } \\
\text { 2010) }\end{array}$ \\
\hline $\begin{array}{l}\text { Clinical } \\
\text { benefits for } \\
\text { patients }\end{array}$ & $\begin{array}{l}\text { A positive effect of a } \\
\text { therapeutic intervention; the } \\
\text { agent demonstrates an } \\
\text { improvement in survival } \\
\text { compared with no therapy or } \\
\text { to a known effective therapy, } \\
\text { equivalence or noninferiority } \\
\text { to a known effective } \\
\text { treatment, or, in some cases, } \\
\text { a clear improvement in time } \\
\text { to disease progression } \\
\text { together with an } \\
\text { improvement in symptoms or } \\
\text { QoL3 for the patient } \\
\text { (Schilsky 2002) }\end{array}$ & $\begin{array}{l}\text { Health } \\
\text { outcome } \\
\text { measures: a } \\
\text { positive effect } \\
\text { of a } \\
\text { therapeutic } \\
\text { intervention } \\
\text { (improvement } \\
\text { in patient's } \\
\text { mobility, } \\
\text { improvement } \\
\text { in adherence } \\
\text { to the } \\
\text { treatment } \\
\text { plan and } \\
\text { behavioral } \\
\text { intervention) }\end{array}$ & $\begin{array}{l}\text { (Morton and } \\
\text { Wiedenbeck } \\
\text { 2009; Lanham et } \\
\text { al. 2012; } \\
\text { Chaudhry et al. } \\
\text { 2006; } \\
\text { Greenspun } \\
\text { 2012; Godzweig } \\
\text { et al. 2009, } \\
\text { Police et al. } \\
\text { 2011, Miller and } \\
\text { Sim 2004; } \\
\text { Shields et al. } \\
\text { 2007) }\end{array}$ \\
\hline $\begin{array}{l}\text { Privacy/ } \\
\text { Security }\end{array}$ & $\begin{array}{l}\text { Protection of personal } \\
\text { information }\end{array}$ & $\begin{array}{l}\text { Protection of } \\
\text { personal } \\
\text { information }\end{array}$ & $\begin{array}{l}\text { (Witter 2009; } \\
\text { Jian et al. 2012; } \\
\text { Lim et al. 2011; } \\
\text { Lustria et al. } \\
\text { 2011) }\end{array}$ \\
\hline
\end{tabular}


Table 28 Taxonomy of model criteria of Interpersonal perspective

\begin{tabular}{|c|c|c|c|}
\hline Criteria & Descriptions & $\begin{array}{l}\text { Definitions for } \\
\text { model }\end{array}$ & References \\
\hline $\begin{array}{l}\text { Efficiency of } \\
\text { Communica } \\
\text { tion and } \\
\text { Patient } \\
\text { Access }\end{array}$ & $\begin{array}{l}\text { Connecting people; } \\
\text { receiving information } \\
\text { through individual and } \\
\text { social prism; transferring } \\
\text { the health data through } \\
\text { interpersonal } \\
\text { relationships; the ability } \\
\text { of the provider to elicit } \\
\text { and understand patient } \\
\text { concerns, to explain } \\
\text { healthcare issues and to } \\
\text { engage in shared } \\
\text { decision-making if } \\
\text { desired. }\end{array}$ & $\begin{array}{l}\text { The ability of the } \\
\text { wearable solution } \\
\text { to improve } \\
\text { interpersonal } \\
\text { connectivity } \\
\text { (among healthcare } \\
\text { team members and } \\
\text { the patient) and } \\
\text { access to patients } \\
\text { as part of remote } \\
\text { health care model. }\end{array}$ & $\begin{array}{l}\text { (Cresswell and } \\
\text { Sheikh 2012; } \\
\text { Beckett et al. } \\
\text { 2011; Wu et al. } \\
\text { 2007; Yu and } \\
\text { Gagnon 2009; } \\
\text { Yarbrough and } \\
\text { Smith 2007; } \\
\text { Chen and Hsiao } \\
\text { 2012; Frambach } \\
\text { and Schillewaert } \\
\text { 2002) }\end{array}$ \\
\hline $\begin{array}{l}\text { Information } \\
\text { Sharing }\end{array}$ & $\begin{array}{l}\text { The ability of new } \\
\text { technological solutions } \\
\text { to provide and perhaps } \\
\text { improve the important } \\
\text { flow of information } \\
\text { through the system } \\
\text { among various levels in } \\
\text { the organization and } \\
\text { outside. }\end{array}$ & $\begin{array}{l}\text { The ability of new } \\
\text { technological } \\
\text { solutions to share } \\
\text { information with } \\
\text { various internal and } \\
\text { external healthcare } \\
\text { providers and } \\
\text { shared decision- } \\
\text { makers. }\end{array}$ & $\begin{array}{l}\text { (Beckett et al. } \\
\text { 2011; Wu et al. } \\
\text { 2007; Yarbrough } \\
\text { and Smith 2007; } \\
\text { Chen and Hsiao } \\
\text { 2012; Cheng } \\
\text { 2012; Yang } \\
\text { 2004; Dünnebeil } \\
\text { et al. 2012) }\end{array}$ \\
\hline $\begin{array}{l}\text { Educational } \\
\text { benefits and } \\
\text { new } \\
\text { knowledge } \\
\text { creation }\end{array}$ & $\begin{array}{l}\text { The ability to contribute } \\
\text { to the creation and } \\
\text { dispersion of new } \\
\text { knowledge in a hospital } \\
\text { setting, while } \\
\text { contributing to } \\
\text { educational benefits for } \\
\text { the parties involved. }\end{array}$ & $\begin{array}{l}\text { Contributing to } \\
\text { educational } \\
\text { benefits and new } \\
\text { knowledge creation } \\
\text { for the parties } \\
\text { involved. }\end{array}$ & $\begin{array}{l}\text { (Degoulet et al., } \\
\text { 1995; Van } \\
\text { Schaik et al. } \\
\text { 2004; Jimoh et } \\
\text { al. 2012; Elwyn } \\
\text { et al. 2000; } \\
\text { Bentley et al. } \\
\text { 2007; Lustria et } \\
\text { al. 2011, } \\
\text { Ammenwerth et } \\
\text { al. 2004) }\end{array}$ \\
\hline
\end{tabular}




\subsubsection{Research focus}

The case alternatives for the model are represented with the selection of medical-grade product innovations - medical devices in the area of mobility tracking for post-spine procedures/surgeries. Those health monitoring devices have medical-grade sensor technology for mobility (accelerometers, gyroscopes. magnetometers) in addition to the network of other vitality-measuring sensors. Those devices prevail in terms of design, efficiency, accuracy, privacy and uniqueness. Thus, the research focus will be evaluation of technology adoption potential of medical-grade wearable devices by physicians. Figure 12 depicting research focus for the study is presented below: 


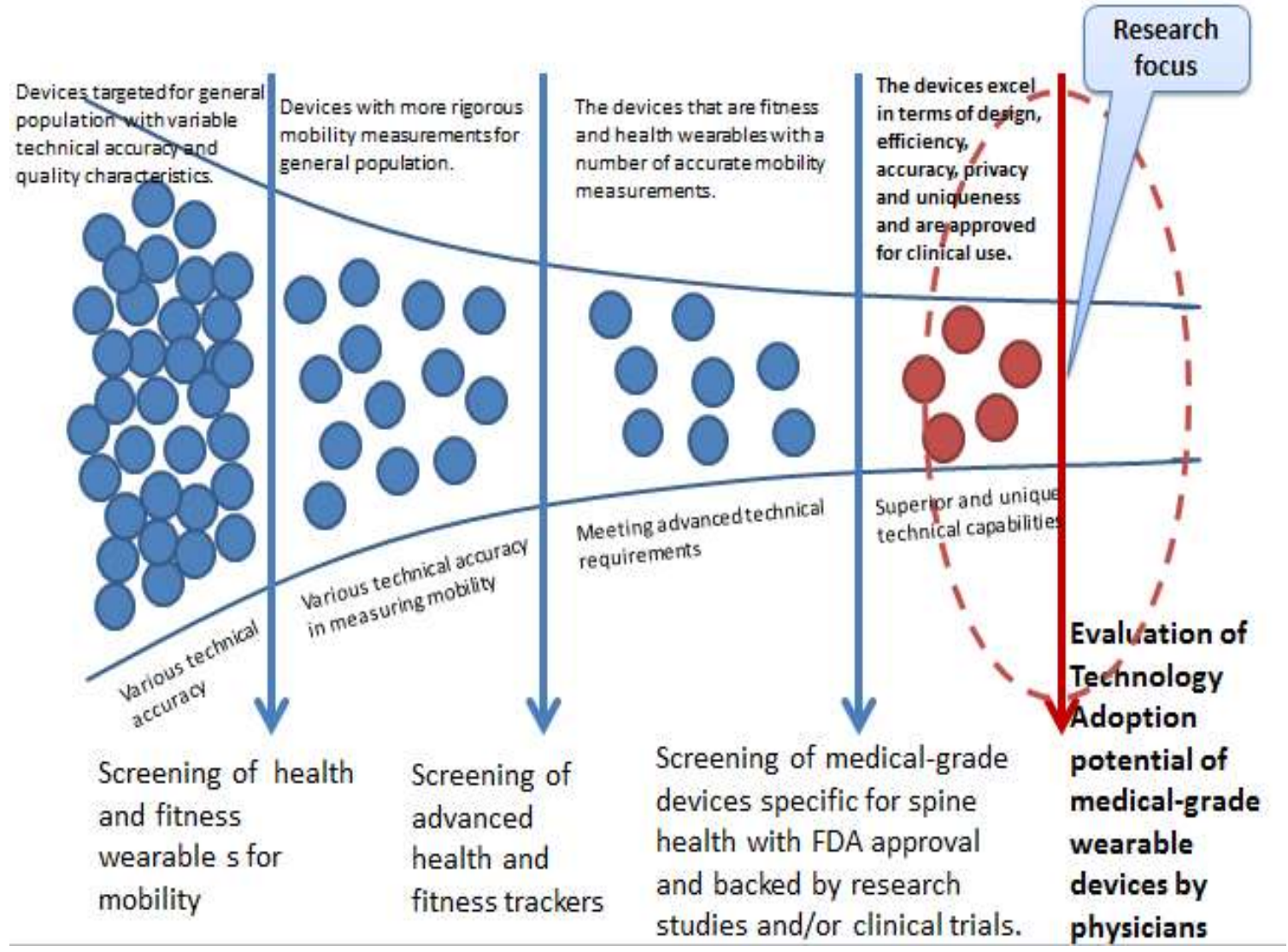

Figure 12 Research focus of this study

\subsubsection{Alternatives - wearable sensor devices}

Wearable products alternatives for preliminary model:

VITALITI by Cloud DX wearable vital sign monitor measures ECG, heart rate, oxygen saturation, respiration, core body temperature, blood pressure, movement, steps and posture and has advanced power management that enables 72 hours of comfortable wear. The sensors of this wearable include electrocardiograph, photoplethysmograph, core thermometer and 3-axis accelerometer. VITALITI monitor is a part of VITALITY platform of four 
interdependent wireless devices, which connect to an advanced mobile app running on a smartphone (Cloud DX 2017).

VitalPatch by VitalConnect comes in a form of $11 \mathrm{~g}$ adhesive

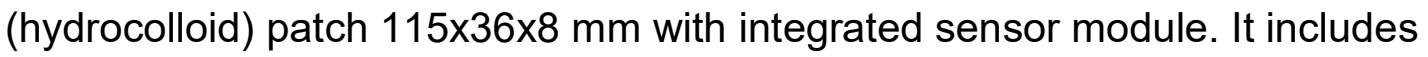
ECG electrodes for heart rate detection, 3-axis MEMS accelerometer for motion detection and thermistor for skin temperature measurement. It is FDA cleared, CE marked, ISO 13485 certified and CMDR registered with disposable zinc air battery (96 hours battery life). It monitors eight vital signs (single-lead ECG, heart rate, heart rate variability, respiratory rate, skin temperature, body posture, fall detection, activity) continuously in real time and care givers and hospital staff can access the information in a consolidated view on a mobile platform (VitalConnect 2017).

Valedo by Hocoma -- back pain therapy medical device with constant feedback and motivation through tracking performance and progress sharing 3D gyroscope $+3 D$ accelerometer $+3 D$ magnetometer (a continuous and precise 9axis vector calculation of movement angles and accelerations). The wireless motion sensors capture body movements in real time and in 3D with the latest Bluetooth technology. Therapeutic exercises are monitored to be executed correctly by playing motivating games based on movement science and physiotherapy while the patients' performance could be tracked and shared on iOS and Android device (Hocoma 2017). 
Opal by ADPM, Inc. -- research-grade wearable sensor solution (3-axis accelerometer +3 -axis gyroscope +3 -axis) magnetometer with robust software development kit built for measuring activity level, tremor, intervention response, gait, balance, diurnal patterns and turning. Opal is made of PC-ABS plastic and glass, weighs less than 25 grams with battery, with dimensions of 43.7×39.7x13.7 mm. Opal has a high sampling rate $(20-200 \mathrm{~Hz})$, robust software development kit, wireless synchronization for up to 24 Opals on one wireless network, can access raw kinematic data, sync with other systems, and provides $8-50$ hours of battery life. The device is able to stream data in real time to a local computer or log data on board each Opal and download later (8 Gb internal storage). Opals could be synchronized with third-party systems, such as optical motion capture systems, EMG, or gait mats (ADPM 2017).

Metria IH1 -- health monitoring device, developed by Vancive Medical Technologies. Metria IH1 is a disposable lifestyle assessment system that collects and reports multiple parameters for health, fitness and wellness purposes. It collects and reports the following parameters for up to 7 days*: physical activity, activity level, calories burned, sleep duration and quality. Sensors include 3-axis accelerometer, skin temperature, near body temperature and galvanic skin response (GSR) (Wearable Technology 2014).

The preliminary model is shown in the diagram below (Figure 13): 


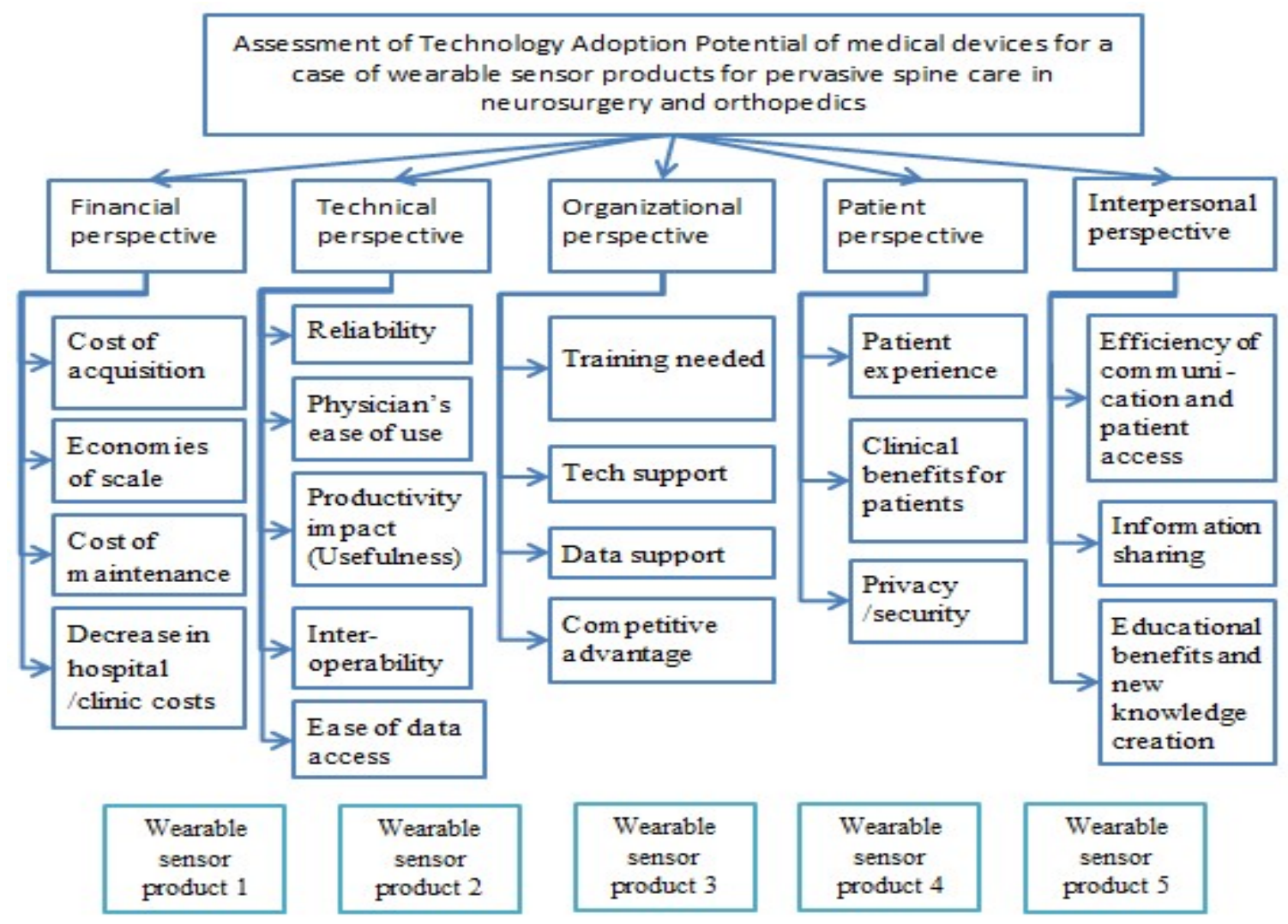

Figure 13 Preliminary model of assessment of Technology Adoption Potential of medical devices.

\subsection{Model Validation}

Validation tests an adequate (sufficient) representation of the model elements and their relationships of the actual systems it's referring to with their underlying importance to model's planned experiments (Greenberger et al. 1976; Labys 1982). Rigorous standards and model credibility are ensured by validation in research studies (Robert Wood Johnson Foundation 2010).

Content validity instrument was constructed in Qualtrics software. Experts from academia and industry were chosen for expert validation with snowballing 
method. Thirty experts provided evaluations of validation and quantification in this study.

The experts are distinguished in their fields of expertise with numerous publications in peer-reviewed journals in their respected fields. The experts are given an opportunity to get acquainted with the research background information, the measurement procedure and other documentation or clarifying information prior to making their judgments.

The experts were contacted via email with a letter that contained a link to the consent letter and validation. The experts were in 7 panels and asked to provide their expert opinion to validate criteria of the model pertaining to their field of expertise. The validation process consisted of validating the perspectives, criteria and alternatives of the model on the yes and no scale. The approval by at least of $75 \%$ of experts means that the variable is approved by the expert panel. The sample of the validation instrument is provided in Appendix B. 
Below are the Figures (Figure 14, Figure 15, Figure 16, Figure 17, Figure 18, Figure 19 and Figure 20) with validation results.

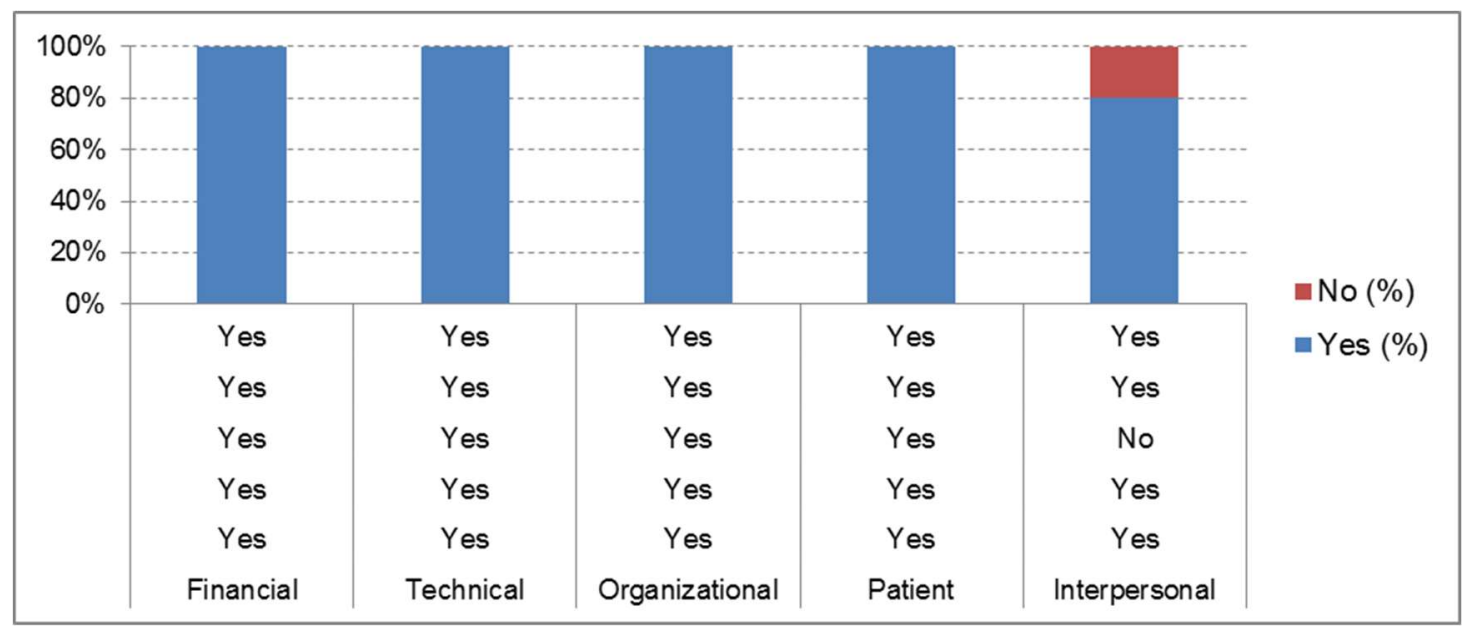

Figure 14 Validation of the model Perspectives

According to the content validation results of panel 1 , all of the perspectives got finalized for the model. Interpersonal perspective received one 'no', which is still within the 'cut-off' value of $75 \%$. 


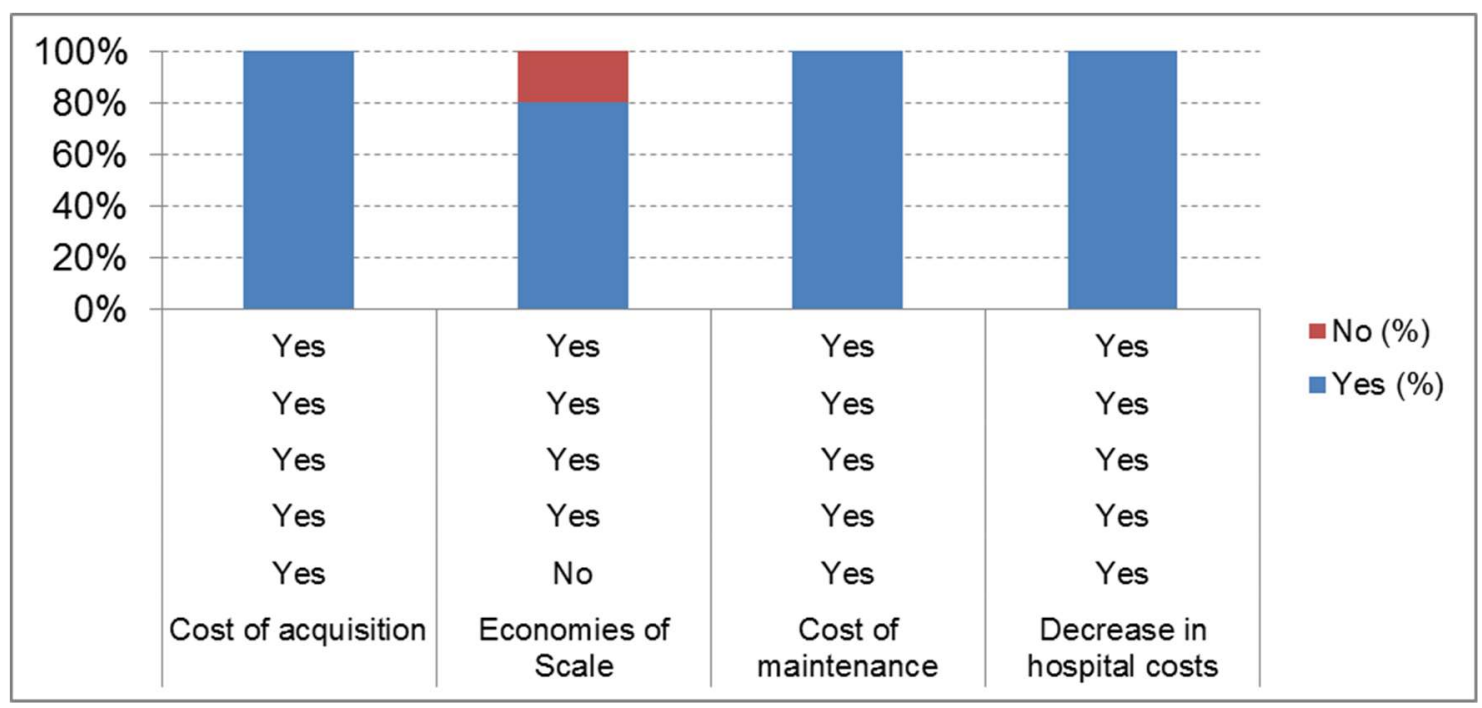

Figure 15 Validation of Financial criteria - Panel 2

Experts of validation panel 2 approved all the Financial criteria of the model. One of the experts felt that Economies of Scale should not be a part of the model.

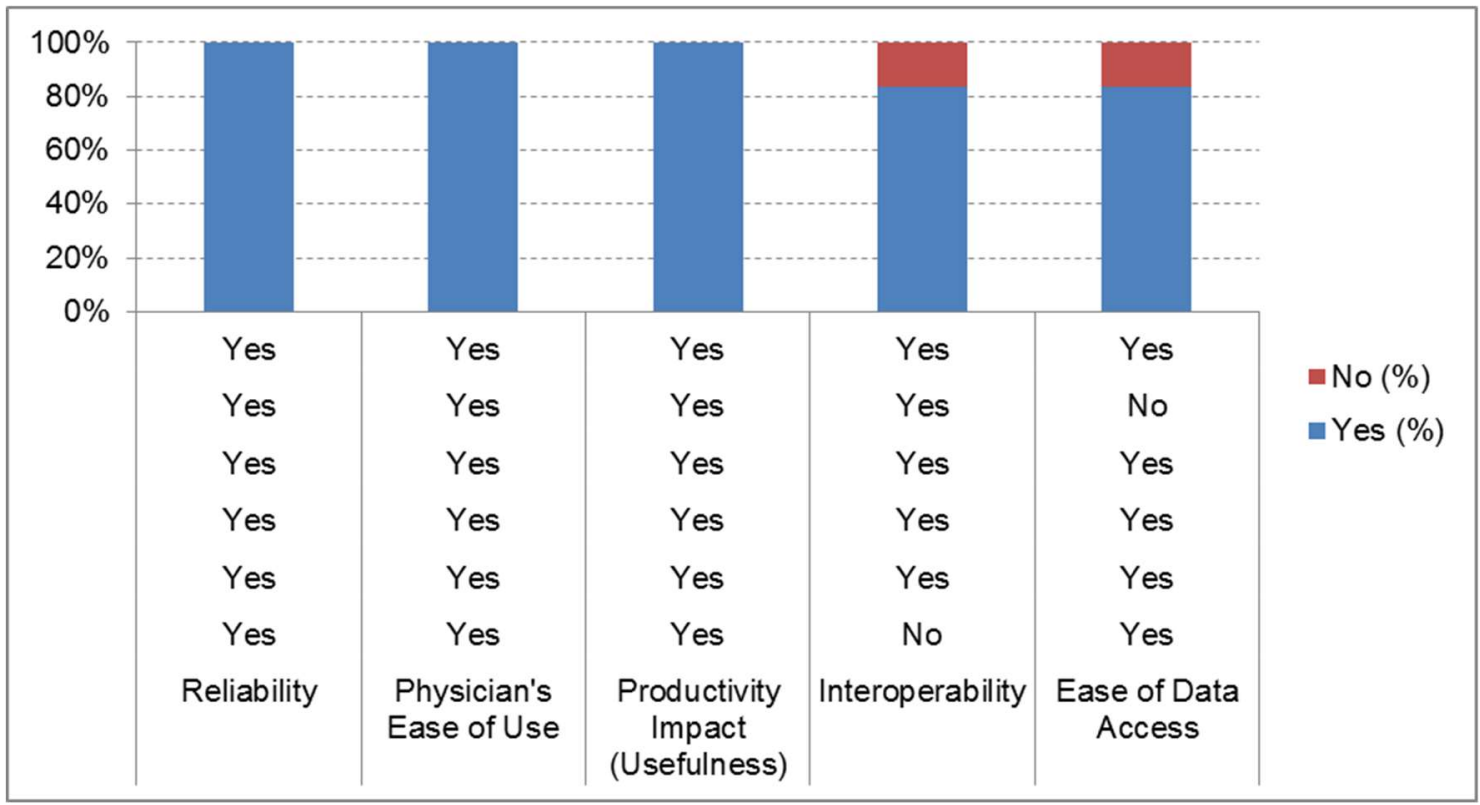

Figure 16 Validation of Technical criteria - Panel 3 
Experts of validation panel 3 approved all Technical criteria. One of the experts gave a 'no' to Interoperability criteria, while another expert felt that Ease of Data Access criteria should not be in the model.

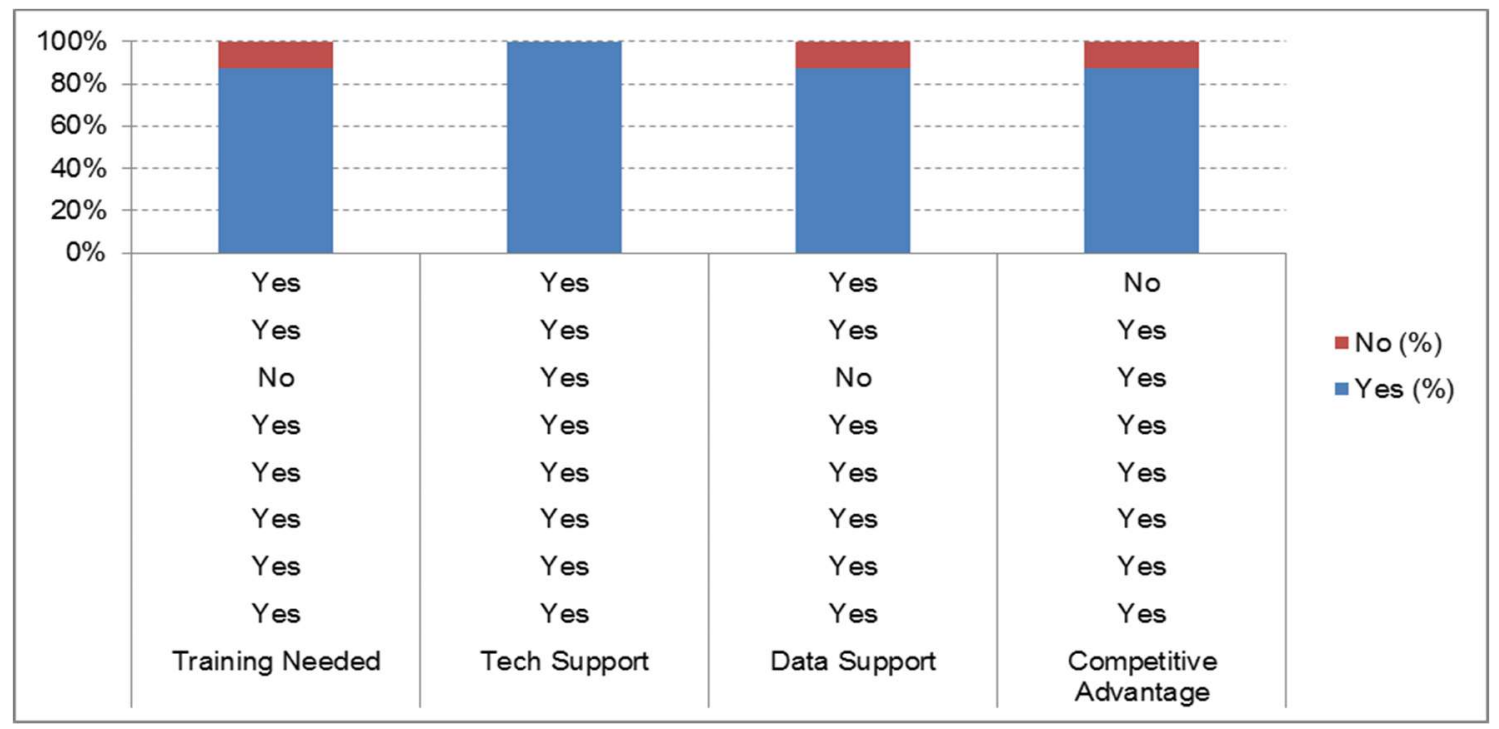

Figure 17 Validation of Organizational criteria - Panel 4

All of the Organizational criteria have been approved by validation panel 4 . 


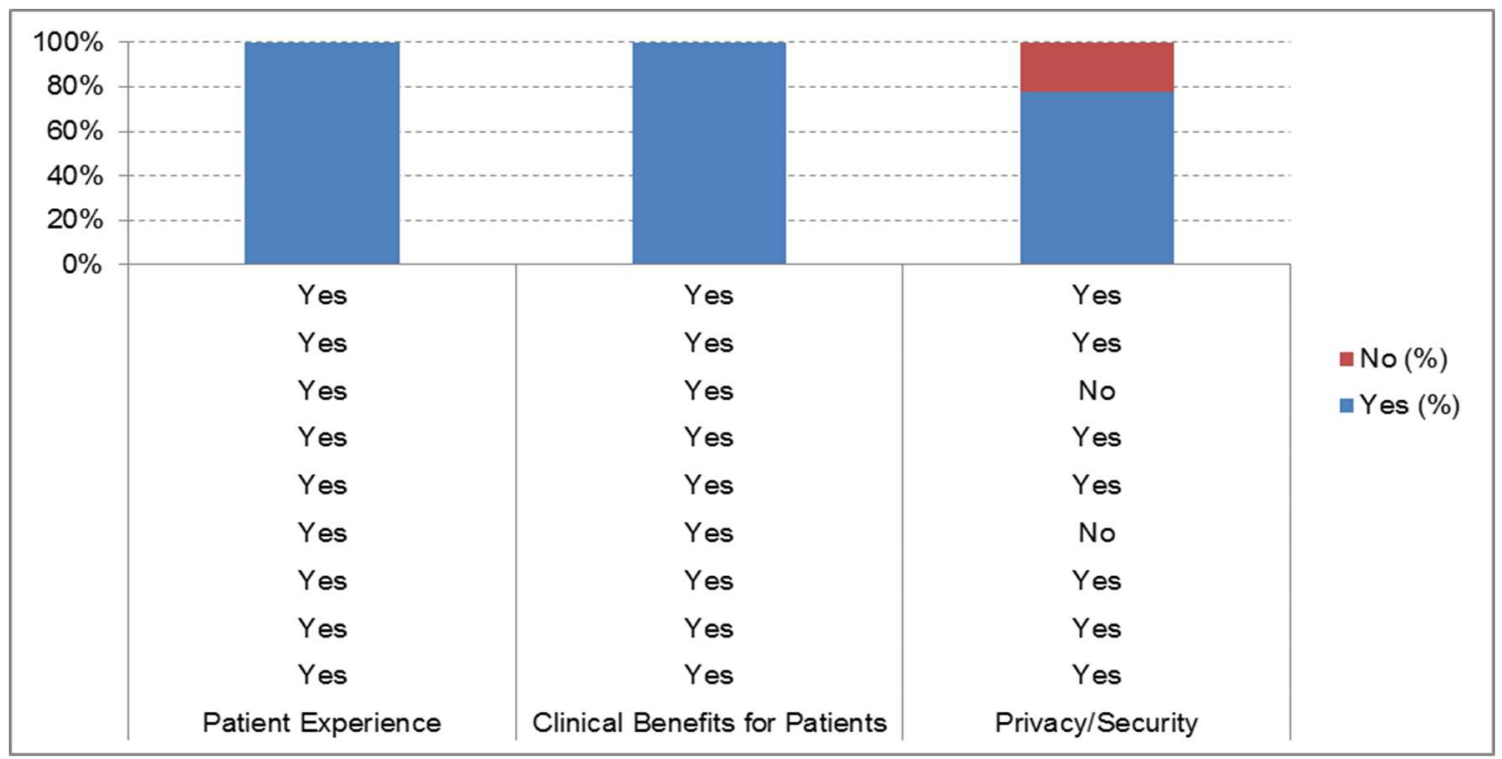

Figure 18 Validation of Patient criteria - Panel 5

Patient criteria have been approved by 9 experts of validation panel 5 .

Two experts felt that Privacy and Security should not be a part of the model. 


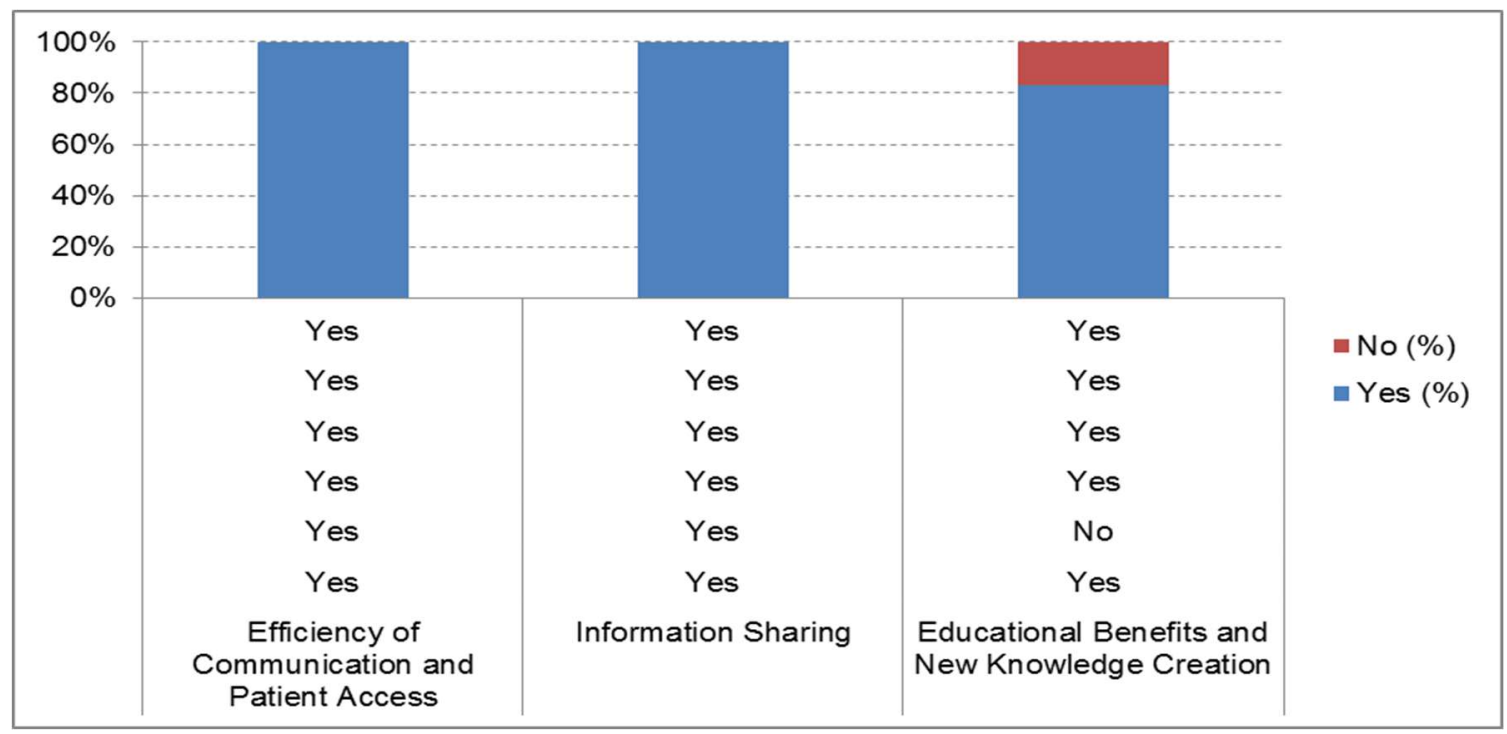

Figure 19 Validation of Interpersonal criteria - Panel 6

Panel 6 experts validated all of the criteria in Interpersonal perspective.

Educational Benefits and New Knowledge Creation criterion got $83 \%$ of approval, which is above the threshold of $75 \%$.

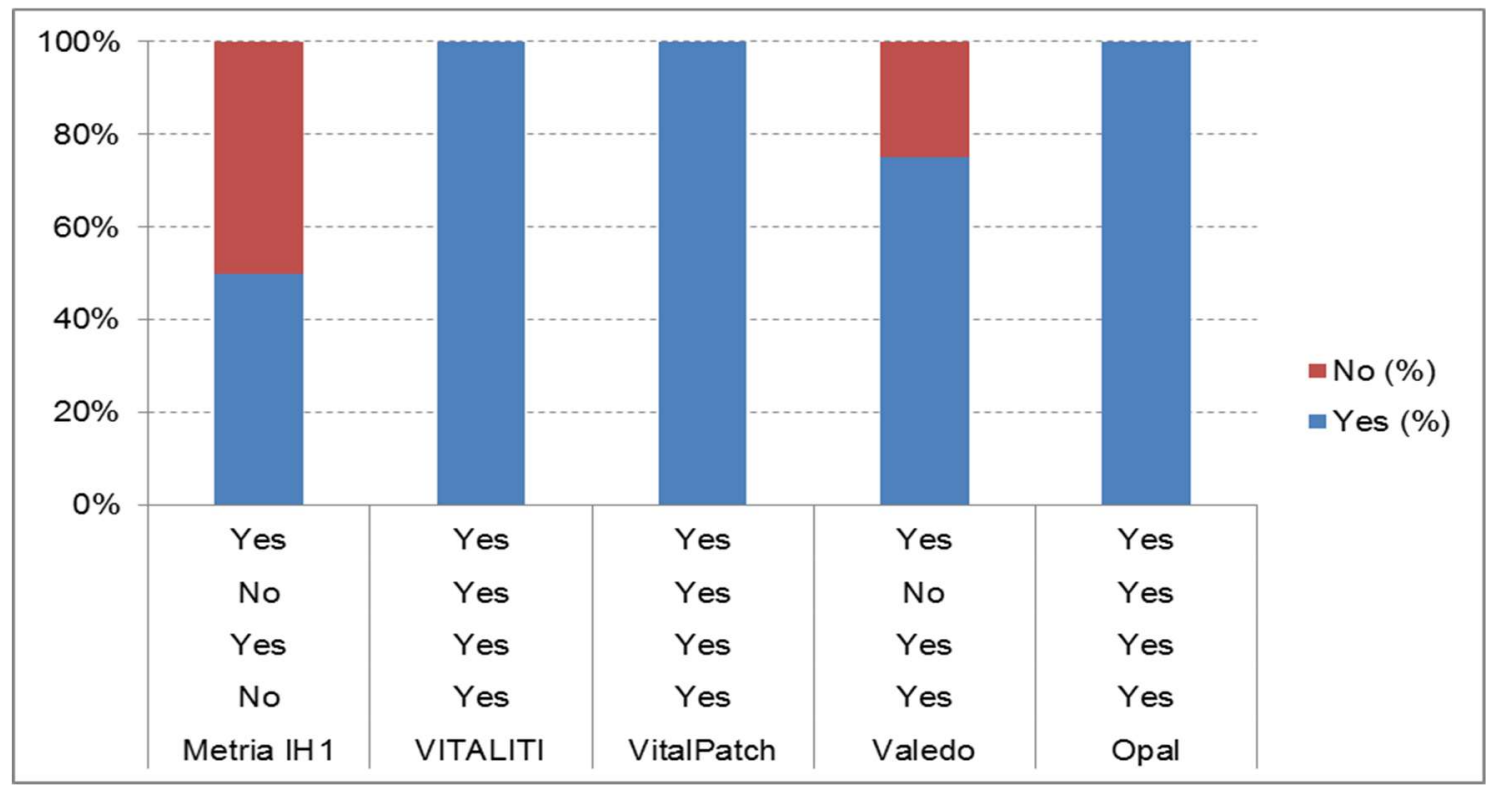


Figure 20 Validation of model Alternatives - Panel 7

All of the model alternatives, except Metria IH1 (50\% of positive responses) were approved by the experts for our model. Therefore, the finalized model will have 4 alternatives: VITALITI, Valedo, Opal and VitalPatch. It is also important to note that VitalPatch used to be HealthPatchMD when it was validated, but then the company-developer renamed it to VitalPatch. In this research the name VitalPatch is used throughout.

Aggregated results are shown in Table 29 below. 
Table 29 Aggregated validation results

\begin{tabular}{|l|l|l|}
\hline Panels & Criteria & $\begin{array}{l}\text { Validation } \\
\text { (Positive } \\
\text { response } \\
\%)\end{array}$ \\
& & 100.00 \\
Panel 1 & Financial & 100.00 \\
& Technical & 100.00 \\
& Organizational & 100.00 \\
& Patient & 80.00 \\
\hline Interpersonal & 100.00 \\
Panel 2 & Cost of acquisition & 80.00 \\
& Economies of scale & 100.00 \\
& Cost of maintenance & 100.00 \\
\hline Panel 3 & Decrease in hospital costs & 100.00 \\
(Technical) & Reliability & 100.00 \\
& Physician's Ease of Use & 100.00 \\
& Productivity Impact (Usefulness) & 83.33 \\
& Interoperability & 83.33 \\
\hline Eanel 4 & Training Needed & 87.50 \\
(Organizational) & Tech Support & 100.00 \\
& Data Support & 87.50 \\
& Competitive Advantage & 87.50 \\
\hline Panel 5 & Patient Experience & 100.00 \\
(Patient) & Clinical Benefits & 100.00 \\
& Privacy/Security & 77.78 \\
\hline Panel 6 & Efficiency of Communication and Patient Access & 100.00 \\
(Interpersonal) & Information Sharing & 100.00 \\
& Educational Benefits and New Knowledge & 83.33 \\
& Creation & \\
\hline Panel 7 & Metria IH1 & 50.00 \\
(Alternatives) & VITALITI & 100.00 \\
& VitalPatch (formerly HealthPatchMD) & 100.00 \\
& Valedo & 75.00 \\
& Opal & 100.00 \\
\hline
\end{tabular}

\subsection{Finalized Assessment Model}

After the model has been validated for content, the research model was modified to reflect the expert opinions. The finalized model, pictured in the Figure 
below, consists of 4 levels: mission level, perspectives level, criteria level, and wearable sensor products (alternatives) level. The first level is the mission of the study and is self-explanatory: assessment of technology adoption potential of medical devices for a case of wearable sensor products for pervasive spine care in neurosurgery and orthopedics.

Five perspectives were chosen based on an extensive literature review and experts. The criteria of perceived usefulness and perceived ease of use, are discussed in the literature review, are based on the elements of the Technology Acceptance Model. Since the above-described research indicates that the acceptance of the technology is based on perceptions of users, those criteria were included in the model. It is assumed that the hardware and software systems or their building components have legal compliance. Finalized assessment model is presented in Figure 21 below: 


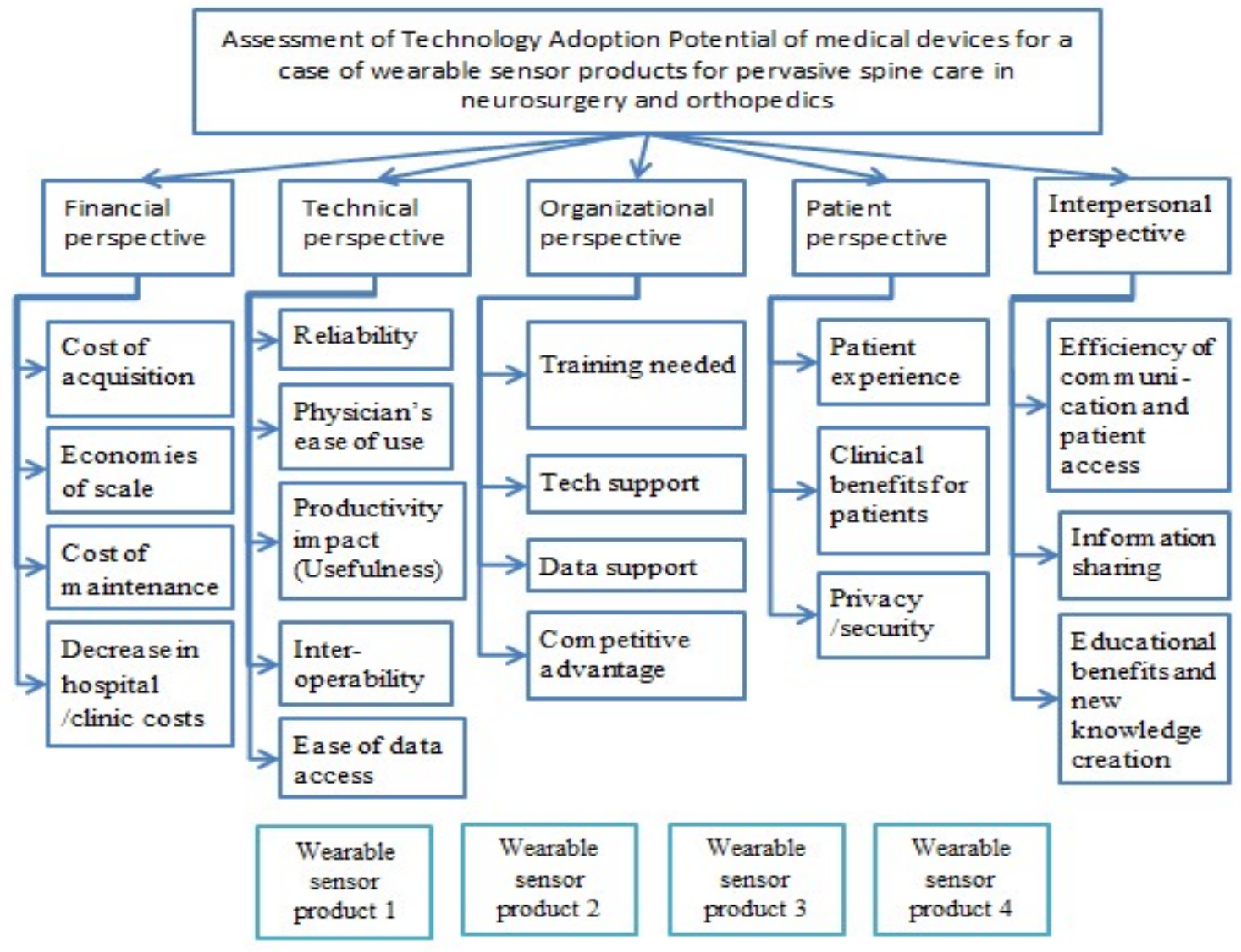

Figure 21 Finalized Assessment model

\subsubsection{Model mission}

The first level is the mission of the study and is self-explanatory: assessment of technology adoption potential of medical devices: case of wearable sensor products for pervasive care in neurosurgery and orthopedics.

\subsubsection{Model perspectives and criteria}

Below are the five perspectives and their criteria with definitions. 
Financial perspective mentioned in the literature from rising costs of healthcare to high costs of systems implementation and management as well as loses from unsuccessful software applications. It encompasses the importance of financial benefits to the organization that a certain technology selection could bring. The following are the financial perspective criteria:

1. Cost of acquisition - expenses associate with the purchase of the wearable sensor technology solution (medical device).

2. Savings due to the economies of scale -- savings to the department and clinic due to obtaining large amounts of a particular kind of wearable technological solution for their patients (provided by the suppliers and by the larger pool of patients served by a wearable medical device).

3. Cost of maintenance - costs associated with maintenance of the system of the wearable technological solution.

4. Decrease in hospital/clinic costs - decrease in overall expenses incurred by hospital in providing services due to the technology acquisition.

Technical perspective represents the value of a technology in a healthcare setting from the view of the importance of technical capabilities improvement. The following criteria represent technical perspective:

1. Reliability - a characteristic of a wearable sensor technological solution providing accurate measurements and analytic reports; consistent and stable performance, free of technical issues and problems. 
2. Physician's ease of use - physician's intuitive and effortless interaction with the wearable sensor technological solution including the accompanied software, databases and analytics/aggregate reports.

3. Productivity impact (usefulness) - the ability of the complete technological solution to enhance the physician's job performance (enabling to provide better care, diagnoses, etc.).

4. Interoperability - vendors support of consensus standards that enable communication and data exchange of wearable sensor technology solution with clinical software applications and databases.

5. Ease of data access - ability of the wearable sensor solution to provide easy access to data reports and analytics.

Organizational perspective encompasses the criteria that technological solutions should provide in organizational setting, which includes management support, integration, tech and data support as well as competitive advantage it can provide for the healthcare organization. Organizational aspect plays a crucial role in technology assessment. Box et al. (2010) stated that success of health information technology implementation requires a careful balance of technical, clinical and organizational factors.

Cresswell and Sheikh (2012) dedicate an empirical and interpretative review study on organizational issues in HIT adoption and implementation. The following four criteria compose organizational perspective: 
1. Training needed - organized activity aimed at imparting information and /or instructions to help medical staff attain a required level of knowledge or skill related to wearable sensor technological solution.

2. Tech support - a service provided by a hardware or software company that provides medical staff with help and advice about the wearable sensor technology solution.

3. Data support - support with overall data integration: formats, standards, changes, downloads, updates, changes etc.

4. Competitive advantage - an advantage over competitors gained by hospitals/clinics offering patients greater value by providing greater benefits and services as results of the wearable sensor technology implementation.

Patient perspective comprises of criteria that reflect physician's view on what technological solution needs to provide like satisfactory patient experience, clinical benefits and privacy and security issues. The criteria are below:

1. Patient experience - overall patient's satisfaction with using a wearable medical device.

2. Clinical benefits for patients - health outcome measures: a positive effect of a therapeutic intervention (improvement in patient's mobility, improvement in adherence to the treatment plan and behavioral intervention).

3. Privacy/security - protection of personal information. 
Interpersonal perspective reflects the importance of sharing and communication among various levels in the organization and outside; the ability of the technological solution to provide that capability and perhaps improve the communication and important flow of information. The operational definition of interpersonal communication is "the ability of the provider to elicit and understand patient concerns, to explain healthcare issues and to engage in shared decisionmaking if desired (Beaulieu et al., 2011). Interpersonal perspective has some elements of social, organizational and personal dynamics (Cresswell and Sheikh, 2012). The criteria definitions are listed below:

1. Efficiency of communication and patient access - the ability of the wearable solution to improve interpersonal connectivity (among healthcare team members and the patient) and access to patients as part of remote health care model.

2. Information sharing - the ability of new technological solutions to share information with various internal and external healthcare providers and shared decision-makers.

3. Educational benefits and new knowledge creation - contributing to educational benefits and new knowledge creation for the parties involved.

\subsubsection{Wearable sensor products}

Four wearable sensor products were validated for the analysis (Table 30): 
Table 30 Wearable sensor products and their sensor modules

\begin{tabular}{|l|l|}
\hline Wearable sensor product & Sensor module \\
\hline VITALITI by Cloud DX & $\begin{array}{l}\text { 3-axis accelerometer and additional sensors } \\
\text { for measuring vitality (electrocardiograph, } \\
\text { photoplethysmograph, core thermometer, } \\
\text { oscillometric cuff (BP), microphone (coughs), } \\
\text { spirometer module, wireless camera) }\end{array}$ \\
\hline VitalPatch by VitalConnect & $\begin{array}{l}\text { 3-axis MEMS accelerometer to detect motion, } \\
\text { ECG electrodes to detect heart rate, thermistor } \\
\text { to detect skin temperature }\end{array}$ \\
\hline Valedo by Hocoma & $\begin{array}{l}\text { 3D gyroscope + 3D accelerometer + 3D } \\
\text { magnetometer (a continuous and precise 9- } \\
\text { axis vector calculation of movement angles } \\
\text { and accelerations }\end{array}$ \\
\hline Opal by ADPM, Inc. & $\begin{array}{l}\text { 3-axis accelerometer + 3-axis gyroscope+ 3- } \\
\text { axis magnetometer }\end{array}$ \\
\hline
\end{tabular}

Product specifications are listed below:

- VITALITI by Cloud DX (Cloud DX 2017)

- Medically accurate consumer/clinical vital sign platform;

- Has 3-axis accelerometer and additional sensors for measuring vitality (electrocardiograph, photoplethysmograph, core thermometer, oscillometric cuff (BP), microphone (coughs), spirometer module, wireless camera);

- Mobility measures: step counter, posture monitor, slip \& fall detector;

- The Cloud DX entry in the Qualcomm Tricorder XPRIZE consists of four interdependent wireless devices connecting to an advanced mobile app running on a smartphone. 
- Wearable Vital Sign Monitor

- Wireless Spirotoscope

- In Vitro Diagnostic System

- Pulsewave Health Station

- VitalPatch by VitalConnect (VitalConnect 2017):

- Comprehensive tracking of eight FDA-cleared measurements in a single biosensor patch;

- single-lead ECG; heart rate; heart rate variability; respiratory rate; skin temperature; body posture; fall detection; activity including steps

- Contextual measurement through multiple data streams and strong foundation for data analytics;

- Continuous remote patient monitoring (data is transmitted to the patient provider with ability to observe improvement or degradation on a real-time basis;

- Supports integration with $3^{\text {rd }}$ party services and applications;

- Wireless Bluetooth low energy connectivity to a mobile device or other relay;

- Wi-Fi connection enables fast data delivery from mobile device to the server;

- All data is encrypted and could be stored in a cloud; 
- The lightweight flexible adhesive patch is worn discretely under clothing.

- Valedo by Hocoma (Hocoma 2017):

- Back pain therapy solution;

- Created by medical device company;

- Two highly sensitive Bluetooth motion sensors;

- 3D gyroscope $+3 D$ accelerometer $+3 D$ magnetometer (a continuous and precise 9-axis vector calculation of movement angles and accelerations);

- iOS/Android App with therapeutic back pain exercises;

- Constant feedback and motivation through tracking performance and progress (pdf report), checking precision and accuracy and ability to share the results with the physician.

- Opal by APDM, Inc. (APDM 2017):

Can measure activity level; tremor; intervention response, gait, balance, diurnal patterns, turning:

- High sampling rate

- Robust Software Development kit

- Access raw kinematic data

- Sync with other systems

- 8-50 hours battery life

- Up to 24 Opals on one network 
- Stream or log data

- Gyroscope + accelerometer + magnetometer.

\subsection{Data Collection and Expert Panels Design}

This study recruited a total of 30 experts for validation and quantification. The experts in quantification process were distributed among 7 expert panels for judgement quantification of model variables. Experts were in the panels according to their field of expertise. Experts had an opportunity to serve on several panels if corresponded with their expertise. Experts were chosen from various healthcare industry, sensor technology industry and academia.

Data collection instrument was developed in Qualtrics. Pairwise comparison method was used for judgment quantifications. Data collection instruments were sent to experts as an anonymous link with a password to a personal questionnaire. Before the start of the questionnaire, the experts were prompted to sign and the Letter of Consent. After the expert answered the questionnaire, the data in .csv, .xls and .xml format was stored in Qualtrics software online. Detailed questionnaire instrument in shown in Appendix C. Table 31 shows the expertise and number of experts in each quantification panel. 
Table 31 Expert panels and expertise of the experts

\begin{tabular}{|c|c|c|}
\hline Expert Panels & Expertise & $\begin{array}{l}\text { Number of } \\
\text { experts }\end{array}$ \\
\hline $\begin{array}{l}\text { Panel } 1 \text { - Main } \\
\text { Perspectives }\end{array}$ & $\begin{array}{l}\text { Professor, Doctor, Researcher, Orthopedics, Spine Center } \\
\text { Clinical Informatics, Anesthesiology, Orthopedics, } \\
\text { Rehabilitation } \\
\text { HIS, Clinical Informatics, OHSU } \\
\text { Continuing Care, Kaiser Permanente } \\
\text { VP and ClO, OHSU } \\
\text { Strategic Director, Merck } \\
\text { Division Director, OHSU } \\
\text { Clinical Content and Decision Support Lead, Kaiser } \\
\text { Permanente } \\
\text { Associate Professor, Medical Informatics and Epidemiology, } \\
\text { OHSU }\end{array}$ & 9 \\
\hline $\begin{array}{l}\text { Panel 2- } \\
\text { Financial }\end{array}$ & $\begin{array}{l}\text { HIS, Clinical Informatics, OHSU } \\
\text { Continuing Care, Kaiser Permanente } \\
\text { VP and CIO, OHSU } \\
\text { Strategic Director, Merck } \\
\text { Division Director, OHSU } \\
\text { Clinical Content and Decision Support Lead, Kaiser } \\
\text { Permanente } \\
\text { Professor, Doctor, Researcher, Orthopedics, Spine Center } \\
\text { Clinical Informatics, Anesthesiology, Orthopedics, } \\
\text { Rehabilitation }\end{array}$ & 8 \\
\hline $\begin{array}{l}\text { Panel 3- } \\
\text { Technical }\end{array}$ & $\begin{array}{l}\text { Director, Intel Labs at Intel Corporation } \\
\text { Neurosurgeon, Rebound Orthopedics } \\
\text { Assistant Professor of Research Orthopedics Surgery, Keck } \\
\text { School of Medicine of USC } \\
\text { Associate Professor, Medical Informatics and Clinical } \\
\text { Epidemiology, OHSU } \\
\text { Associate Professor, Medical Informatics and Epidemiology, } \\
\text { OHSU } \\
\text { Continuing Care, Kaiser Permanente } \\
\text { VP and CIO, OHSU } \\
\text { Assistant Professor of Neurological Surgery, School of } \\
\text { Medicine, OHSU } \\
\text { HIS, Clinical Informatics, OHSU } \\
\text { Strategic Director, Merck }\end{array}$ & 10 \\
\hline $\begin{array}{l}\text { Panel } 4 \text { - } \\
\text { Organizational }\end{array}$ & $\begin{array}{l}\text { Associate Professor, Medical Informatics and Clinical } \\
\text { Epidemiology, OHSU } \\
\text { Assistant Professor of Research Orthopedics Surgery, Keck } \\
\text { School of Medicine of USC } \\
\text { Instructor of Neurological Surgery, Division of Skull Base and } \\
\text { Cerebrovascular School of Medicine Physician Assistant, } \\
\text { OHSU } \\
\text { Continuing Care, Kaiser Permanente } \\
\text { VP and CIO, OHSU } \\
\text { Division Director, OHSU } \\
\text { Strategic Director, Merck } \\
\text { Assistant Professor of Neurological Surgery, School of } \\
\text { Medicine, OHSU }\end{array}$ & 9 \\
\hline
\end{tabular}




\begin{tabular}{|c|c|c|}
\hline Expert Panels & Expertise & $\begin{array}{l}\text { Number of } \\
\text { experts }\end{array}$ \\
\hline $\begin{array}{l}\text { Panel } 5 \text { - } \\
\text { Patient }\end{array}$ & $\begin{array}{l}\text { Assistant Professor of Research Orthopedics Surgery, Keck } \\
\text { School of Medicine of USC } \\
\text { Professor, Doctor, Researcher, Orthopedics, Spine Center } \\
\text { Clinical Informatics, Anesthesiology, Orthopedics, } \\
\text { Rehabilitation } \\
\text { Neurosurgeon, Rebound Orthopedics } \\
\text { Continuing Care, Kaiser Permanente } \\
\text { Assistant Professor of Neurological Surgery, School of } \\
\text { Medicine, OHSU } \\
\text { Instructor of Neurological Surgery, Division of Skull Base and } \\
\text { Cerebrovascular School of Medicine Physician Assistant, } \\
\text { OHSU } \\
\text { HIS, Clinical Informatics, OHSU }\end{array}$ & 8 \\
\hline $\begin{array}{l}\text { Panel } 6 \text {-- } \\
\text { Interpersonal }\end{array}$ & $\begin{array}{l}\text { Assistant Professor of Research Orthopedics Surgery, Keck } \\
\text { School of Medicine of USC } \\
\text { Neurosurgeon, Rebound Orthopedics } \\
\text { Instructor of Neurological Surgery, Division of Skull Base and } \\
\text { Cerebrovascular School of Medicine Physician Assistant, } \\
\text { OHSU } \\
\text { Continuing Care, Kaiser Permanente } \\
\text { VP and CIO, OHSU } \\
\text { Strategic Director, Merck } \\
\text { Division Director of Perioperative Services, OHSU } \\
\text { Professor, Doctor, Researcher, Orthopedics, Spine Center } \\
\text { Clinical Informatics, Anesthesiology, Orthopedics, } \\
\text { Rehabilitation }\end{array}$ & 10 \\
\hline Panel 7 & $\begin{array}{l}\text { Neurosurgeon, Rebound Orthopedics } \\
\text { Instructor of Neurological Surgery, Division of Skull Base and } \\
\text { Cerebrovascular School of Medicine } \\
\text { Professor, Doctor, Researcher, Orthopedics, Spine Center } \\
\text { Clinical Informatics, Anesthesiology, Orthopedics, } \\
\text { Rehabilitation } \\
\text { Associate Professor, Medical Informatics and Clinical } \\
\text { Epidemiology, OHSU } \\
\text { Associate Professor, Medical Informatics and Epidemiology, } \\
\text { OHSU } \\
\text { Director, Intel Labs at Intel Corporation } \\
\text { Biosensor Specialist, PhD, Intel Labs at Intel Corporation }\end{array}$ & 8 \\
\hline
\end{tabular}

Experts in each panel were responsible to analyze the perspectives, criteria or alternatives relative to the mission. Experts from Panel 7 were also broken into subpanels according to their expertise. The experts were asked to compare decision variables in a pair-wise fashion. In case the experts had 
difficulty with the terms, they could refer to the explanations for the terms listed.

The detailed descriptions of experts' tasks are presented in the tables (Table 32 and Table 33) below.

Table 32 Quantification panel focus and task

\begin{tabular}{|l|l|l|}
\hline Panel \# & \multicolumn{1}{|c|}{ Focus } & \multicolumn{1}{|c|}{ Main task } \\
\hline Panel 1 & Multiple Perspectives & $\begin{array}{l}\text { pairwise comparison of main wearable } \\
\text { technology assessment perspectives to } \\
\text { reflect the judgement on their relative } \\
\text { importance to overall mission }\end{array}$ \\
\hline Panel 2 & Financial criteria & $\begin{array}{l}\text { comparison the importance of criteria in } \\
\text { 'Financial perspective' according to the } \\
\text { mission }\end{array}$ \\
\hline Panel 3 & Technical criteria & $\begin{array}{l}\text { comparison the importance of criteria in } \\
\text { 'Technical perspective' according to the } \\
\text { mission }\end{array}$ \\
\hline Panel 4 & Organizational criteria & $\begin{array}{l}\text { comparison the importance of criteria in } \\
\text { 'Organizational perspective' according to the } \\
\text { mission }\end{array}$ \\
\hline Panel 5 & Patient criteria & $\begin{array}{l}\text { comparison the importance of criteria in } \\
\text { 'Patient perspective' according to the } \\
\text { mission }\end{array}$ \\
\hline Panel 6 & Interpersonal criteria & $\begin{array}{l}\text { comparison the importance of criteria in } \\
\text { 'Financial perspective' according to the } \\
\text { mission }\end{array}$ \\
\hline Panel 7 & Alternatives level & $\begin{array}{l}\text { ranking of the wearable products for the } \\
\text { Assessment of Technology Adoption } \\
\text { Potential of wearable medical devices for } \\
\text { pervasive spine care in neurosurgery and } \\
\text { orthopedics with respect to the criterion }\end{array}$ \\
\hline
\end{tabular}


Table 33 Subpanels of quantification expert panel 7

\begin{tabular}{|c|l|l|}
\hline Panel \# & \multicolumn{1}{|c|}{ Focus } & \multicolumn{1}{c|}{ Main task } \\
\hline Panel 7.1 & $\begin{array}{l}\text { Alternatives } \\
\text { with respect to } \\
\text { Financial criteria }\end{array}$ & $\begin{array}{l}\text { ranking of the wearable products for the } \\
\text { Assessment of Technology Adoption } \\
\text { Potential of wearable medical devices for } \\
\text { pervasive spine care in neurosurgery and } \\
\text { orthopedics with respect to the criteria in } \\
\text { Financial perspective }\end{array}$ \\
\hline Panel 7.2 & $\begin{array}{l}\text { Alternatives } \\
\text { with respect to } \\
\text { Technical criteria }\end{array}$ & $\begin{array}{l}\text { ranking of the wearable products with } \\
\text { respect to the criteria in Technical } \\
\text { perspective }\end{array}$ \\
\hline Panel 7.3 & $\begin{array}{l}\text { Alternatives } \\
\text { with respect to } \\
\text { Organizational criteria }\end{array}$ & $\begin{array}{l}\text { ranking of the wearable products with } \\
\text { respect to the criteria in Organizational } \\
\text { perspective }\end{array}$ \\
\hline Panel 7.4 & $\begin{array}{l}\text { Alternatives } \\
\text { with respect to } \\
\text { Patient criteria }\end{array}$ & $\begin{array}{l}\text { ranking of the wearable products with } \\
\text { respect to the criteria in Patient perspective }\end{array}$ \\
\hline Panel 7.5 & $\begin{array}{l}\text { Alternatives } \\
\text { with respect to } \\
\text { Interpersonal criteria }\end{array}$ & $\begin{array}{l}\text { ranking of the wearable products with } \\
\text { respect to the criteria in Interpersonal } \\
\text { perspective }\end{array}$ \\
\hline
\end{tabular}




\section{CHAPTER 7. DATA ANALYSIS TOOLS}

\subsection{Hierarchical Decision Modeling Research Software Package}

The Hierarchical Decision Modeling Research Software tool flow provides

a suite of software tools that allows collection of the expert panel responses from Qualtrics and their migration to R in order to perform HDM calculations, analysis, and visualization of the resultant data.

The flow of the research software tool consists of the following steps (Figure 22):

1. Extracting data from Qualtrics with XML file (generated by Qualtrics).

2. Transferring data with Ruby code (created for this research) and two XML criteria mapping files: question number-criteria pair; perspectives-criteriaalternatives (created for this study).

3. Running file in $R$ (created, validated new code and used $R$ packages).

4. Validation of achieved results with PCM software that has been used and validated in HDM studies. 

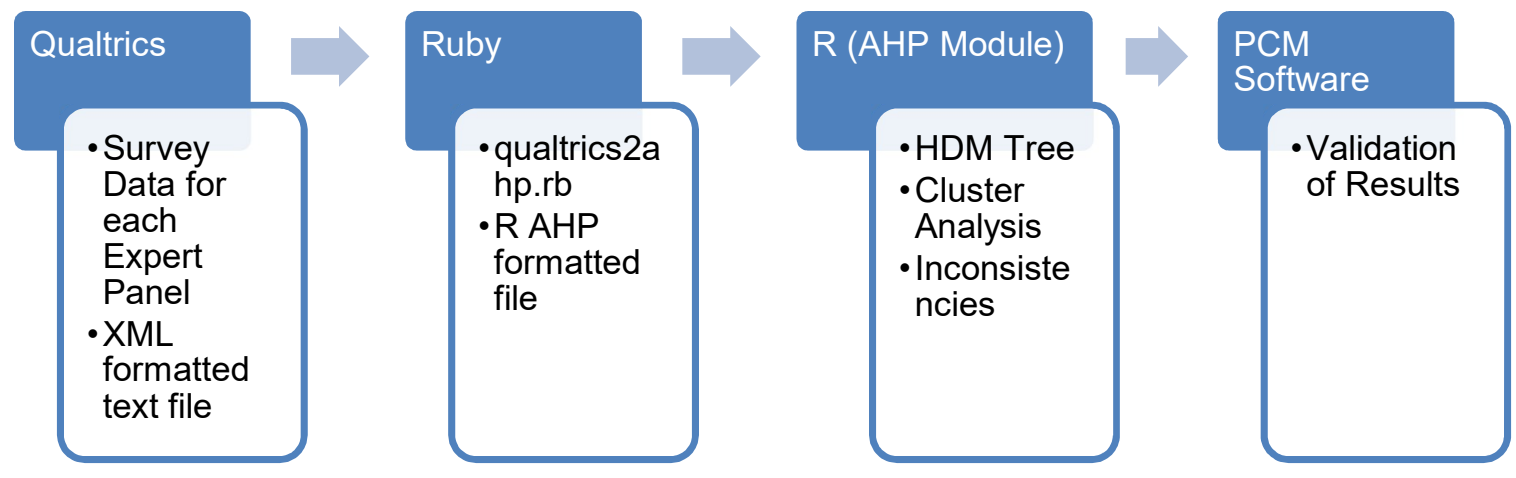

Figure 22 Software tools process flow

\subsection{Software Tool Component Descriptions}

\subsubsection{Qualtrics questionnaire instrument}

Qualtrics provides a web-based questionnaire framework that is used to collect responses for each expert panel in the HDM model. This application provides an XML Schema for the data which can then be downloaded in the form of an XML formatted file for all of the survey responses for each expert panel. This XML data file is then sent to the qualtrics2ahp command line tool for further processing.

\subsubsection{Qualtrics2ahp data processing utility}

The qualtrics2ahp command line tool is written in Ruby and is used to convert the XML data file from Qualtrics into a form useable by the R AHP/HDM analysis module. Although the primary input to the Ruby script is the XML formatted expert panel file, the script also requires two additional XML formatted 
mapping files to understand how the questions in each expert panel are mapped to the HDM data tree model.

The first mapping file defines the relationship between the unique ID (question_ids) in Qualtrics and their associated criteria in the HDM model. The basic structure of this XML file looks like the following:

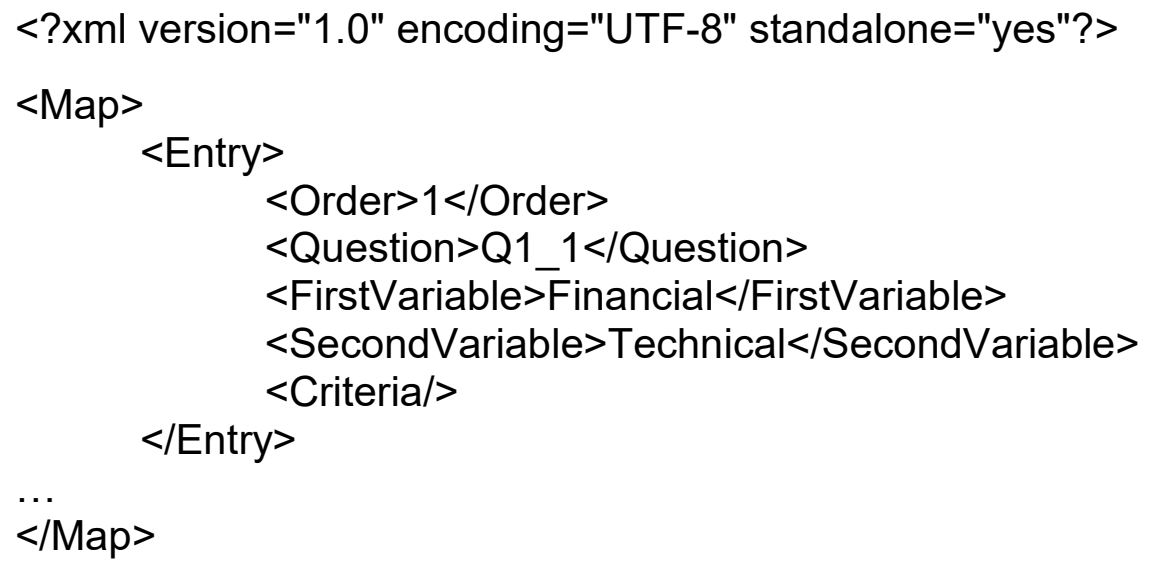

The second XML formatted file maps each perspective to the associated criteria thereby formulating the HDM tree structure required to analyze the panel responses.

$<$ ?xml version="1.0" encoding="UTF-8" standalone="yes"?> $<$ Criteria $>$ 
The output from the qualtrics2ahp Ruby script is a data file in HDM format that can be loaded and processed by the R CRAN AHP package module.

\subsubsection{R CRAN AHP package customized script}

The R portion of the HDM software tool flow loads the HDM file generated by the qualtrics2ahp script and loads the hierarchical decision model tree data in an AHP module for calculation, visualization, and analysis package.

Customization of data processing of our HDM data is controlled by implementing an R script, which can perform the functions of 'Calculate', 'Analyze', and 'Visualize' required for our HDM model. The capability to perform 'Inconsistency' calculations on the individual panels as well as cluster analysis for disagreement checking in this $\mathrm{R}$ script is programmed in as well. The AHP package for $\mathrm{R}$ is available on the CRAN repository (CRAN 2017).

\subsubsection{PCM software}

PCM software is an MS-DOS based software package that was used as a validation step to ensure that the calculated values for inconsistency, disagreement, and other factors in the new tool flow matched the output from a standardized tool that is used across PSU ETM department research. The software requires that you run a 32-bit MS-DOS emulator, like Dos Box, on modern PCs because it is a 16-bit application. Entering the data into PCM is laborious. A newer, more modern tool in $\mathrm{R}$ is more convenient and accurate for data analysis on large data sets. 
By validating our new tool flow against an accepted standard tool for HDM research (running the model in both environments and achieving matching results to the precision of the values from PCM software), I ensured that my implementation of HDM in the new software tool flow matches the results obtained for prior research publications that used the MS-DOS tool. This showed that my data analysis is in agreement with both tools and therefore my implementation is correct to within the degree that PCM is correct.

\subsection{Software Tool Flow Steps}

The following sections describe the steps necessary to analyze a single expert panel from Qualtrics all the way through to analysis in R.

\subsubsection{Saving survey response panel data from Qualtrics to XML}

Survey responses from Qualtrics are saved to an XML formatted file with a very specific naming convention that indicates which panels are included in the file. For example, the file name format looks like the following: WSPA_123.xml.

This indicates that the XML data file contains responses from experts for panels 1, 2, 3. For each survey response there will be a set of these files. Using Computer Science terminology, a regular expression for the file name looks like the following: WSPA_[123]/.

\subsubsection{Using Ruby qualtrics2ahp.rb to convert XML to HDM format}

After each WSPA_ ${ }^{*} . x m l$ file is saved in a subdirectory of the qualtrics2ahp utility named ./survey_data. When run from the command line, the Ruby 
qualtrics2ahp.rb file will search the ./survey_data for all of the WPA_* ${ }^{*}$ xml files for the expert panel that will be analyzed. For example, if you are analyzing all of the panel 1 responses then the script will process all files named ./survey_data/WSPA_ $1^{*} . \mathrm{xml}$. This is done through invoking the command from a Ruby command window as follows: > ruby qualtrics2ahp.rb.

This generates an HDM formatted file named ./panel1.ahp suitable for loading into the $\mathrm{R}$ environment and analyzing.

\subsubsection{Generating HDM in $R$ using AHP module}

The following sequence of commands is then issued in $R$ to further process the panel1.ahp file and analyze the responses from Qualtrics.

In $\mathrm{R}$, issue the following commands:

$>$ ahpFile <- Load(“./panel1.ahp")

$>$ CalculateHDM(ahpFile, panel=1)

This will print out a new HDM formatted file to the console window that will need to be copied and pasted to a new file named panel1.hdm.

$>$ hdmFile <- Load(“./panel1.hdm”)

Now calculate the HDM results and inconsistency values using the following command:

$>$ Calculate(hdmFile)

$>$ Analyze(hdmFile) 


\subsubsection{Analyzing inconsistencies}

Inconsistency values are contained within the newly generated panel1.hdm file. Panel1.hdm file needs to be edited to remove any responses that are outside of the inconsistency bounds. In the future, I plan to make this process either automatically done or to allow the user to choose which responses to eliminate during the CalculateHDM step.

\subsubsection{Analyzing disagreement and cluster analysis in $\mathbf{R}$}

Once 'Calculate()' in $\mathrm{R}$ has been run, Cluster Analysis in $\mathrm{R}$ can be generated on the loaded hdmFile. The following commands are issued:

$>$ cla $<-$ ClusterAnalysis(hdmFile)

$>$ plot(cla)

The above procedure will display the cluster diagram for the panel that was just calculated. 


\section{CHAPTER 8. RESULTS AND DATA ANALYSIS}

The current chapter presents the results of the expert judgement quantification, judgement inconsistencies and group disagreements. Expert judgements were quantified in R and PCM software. The threshold value for inconsistency of 0.1 was based on previous studies (Gerdsri and Kocaoglu 2009: Gerdsri 2005; Iskin 2014; Abotah 2015). In addition, inconsistencies above the value of 0.1 were tested against Root Sum Variance (Abbas 2016) graphs. Cluster dendrograms were used in group disagreements over the value of 0.1 . Discussion of priorities synthesis, sensitivity analysis and scenario analysis is shown here as well.

\subsection{Expert Panel 1}

\subsubsection{Expert panel 1 results}

Experts in expert panel 1 were asked to evaluate relative importance of main technology assessment perspectives with respect to overall mission of assessment of technology adoption potential of medical devices for a case of wearable sensor products for pervasive spine care in neurosurgery and orthopedics. The arithmetic means of the relative importance of main technology assessment perspectives of panel 1 [8 expert opinions] are shown in the following figure (Figure 23): 


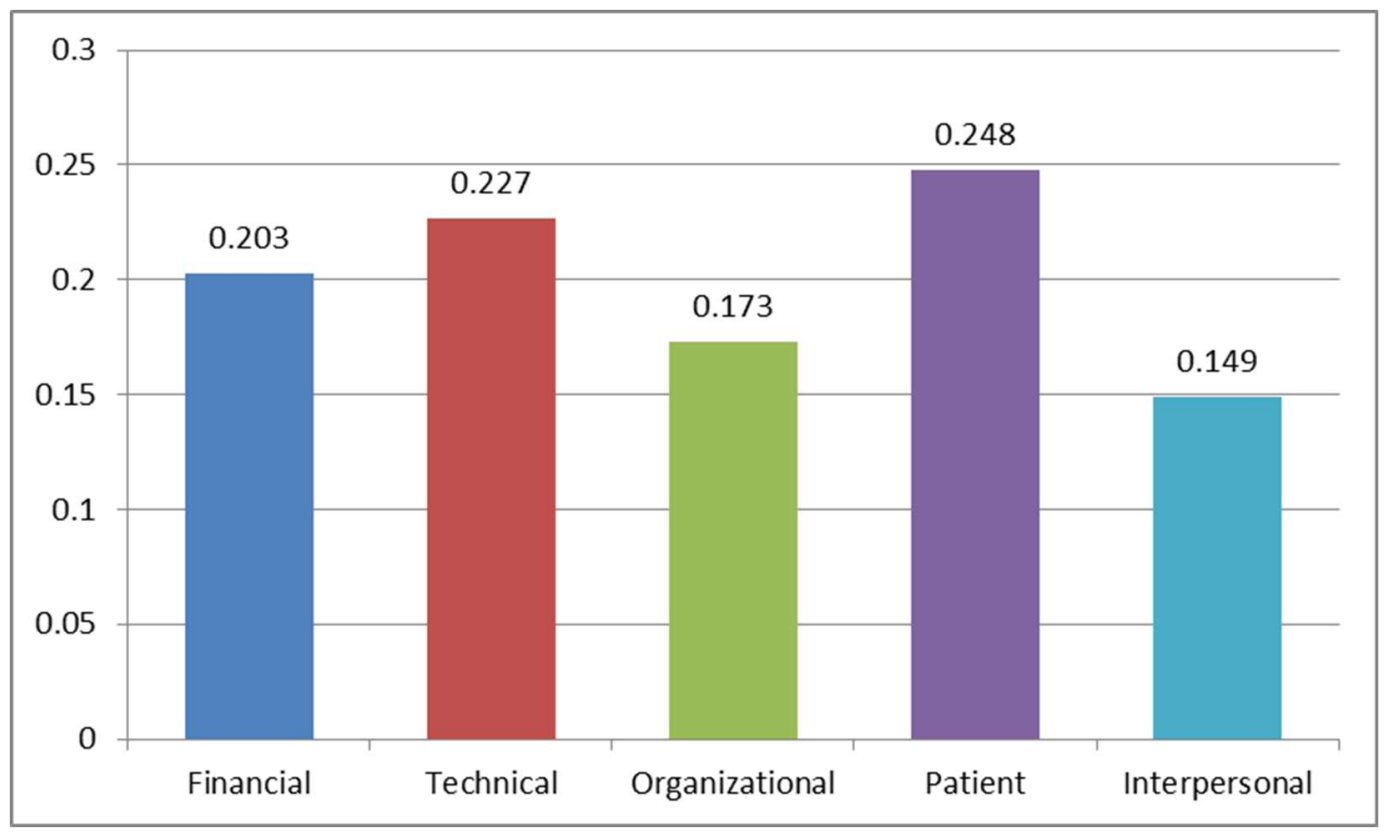

Figure 23 Relative importance of technology assessment perspectives

Quantification of the expert judgements shows that the most important perspective with respect to the mission is Patient perspective (24.8\%), which reflects patient issues of adoption of medical devices like satisfactory patient experience, clinical benefits and privacy/security of information. Technical perspective follows with $22.7 \%$ and financial perspective occupies the third place $(20.3 \%)$. The least important technology assessment perspective is interpersonal $(14.9 \%)$

\subsubsection{Analysis of expert panel 1 results}

Only experts that had an acceptable level of consistency were included in calculations of relative importance of perspectives. Three experts had inconsistency levels above 0.1 and their Root Sum Variance was calculated 
according to (Abbas 2016). The threshold of acceptable inconsistency with RSV value for 5 decision variables at $\alpha=0.05$ is 0.2651 . RSV values for the three experts with inconsistencies of $0.188,0.161$ and 0.118 were $0.421,0.360$ and 0.263 respectively. Since RSV value of 0.263 is below the threshold of 0.2651 , the evaluations of that expert were included in the analysis. There was also some disagreement among the experts $(0.114)$ present and will be discussed in this chapter. Table 34 with individual relative priority weights, aggregated group results, inconsistency levels and group disagreement is presented below.

Table 34 Analysis of expert panel 1 results

\begin{tabular}{|c|c|c|c|c|c|c|}
\hline Panel 1 & Financial & Technical & Organizational & Patient & Interpersonal & Inconsistency \\
\hline Expert 1 & 0.04 & 0.23 & 0.11 & 0.33 & 0.28 & 0.029 \\
\hline Expert 2 & 0.11 & 0.13 & 0.14 & 0.49 & 0.13 & 0.035 \\
\hline Expert 3 & 0.21 & 0.29 & 0.1 & 0.33 & 0.08 & 0.024 \\
\hline Expert 4 & 0.28 & 0.4 & 0.05 & 0.22 & 0.06 & 0.018 \\
\hline Expert 5 & 0.22 & 0.24 & 0.24 & 0.11 & 0.19 & 0.084 \\
\hline Expert 6 & 0.43 & 0.14 & 0.17 & 0.07 & 0.18 & 0.070 \\
\hline Expert 7 & 0.16 & 0.15 & 0.15 & 0.37 & 0.19 & 0.004 \\
\hline Expert 8 & 0.18 & 0.25 & 0.42 & 0.07 & 0.08 & 0.118 \\
\hline Mean & 0.203 & 0.227 & 0.173 & 0.248 & 0.149 & \\
\hline \multicolumn{2}{|c|}{ Disagreement } & & & & & 0.114 \\
\hline
\end{tabular}

Hierarchical agglomerative clustering in $\mathrm{R}$ has been used to identify subgroups within expert panel 1. Two subgroups have been identified with hierarchical cluster analysis and the dendrogram is presented in Figure 24. 


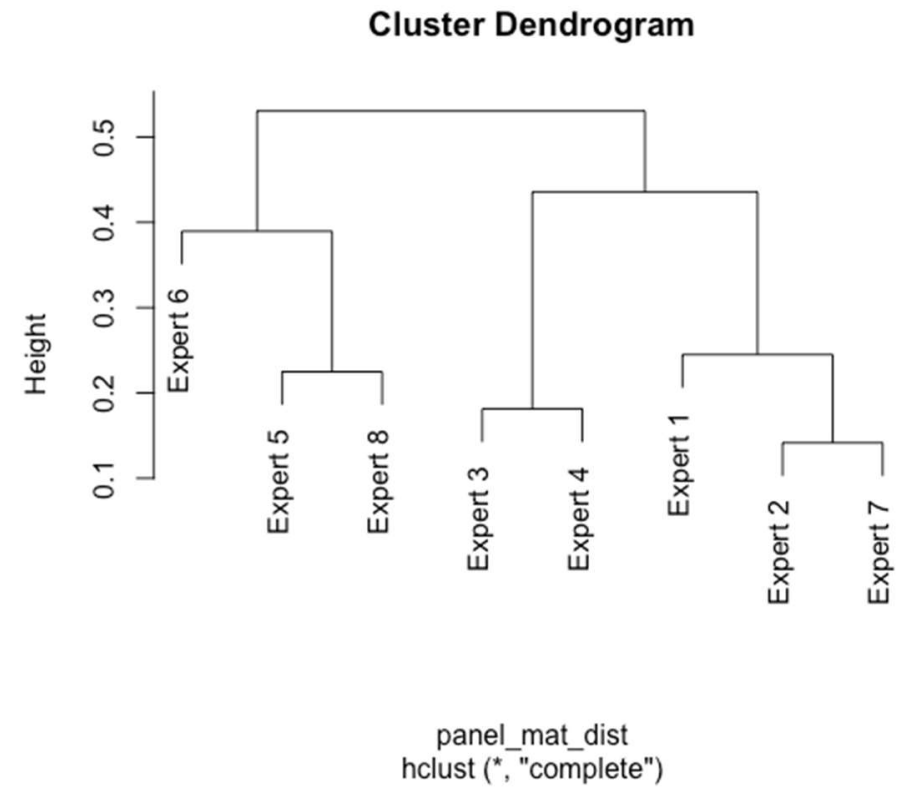

Figure 24 Dendrogram of subgroups of expert panel 1

Group disagreements of subgroup A (experts 5, 6 and 8) and subgroup B (experts 1,2,3,4 and 7) are below the threshold value of 0.1 and are 0.094 and 0.089 respectively. Table 35 and Table 36 show expert relative priorities, inconsistencies, aggregated group results and group disagreements for the two subgroups in panel 1: subgroup A and $B$.

Table 35 Analysis of results in subgroup A of expert panel 1

\begin{tabular}{|l|r|r|r|r|r|r|}
\hline Panel 1 & Financial & Technical & Organizational & Patient & Interpersonal & Inconsistency \\
\hline Expert 5 & 0.22 & 0.24 & 0.24 & 0.11 & 0.19 & 0.084 \\
\hline Expert 6 & 0.43 & 0.14 & 0.17 & 0.07 & 0.18 & 0.070 \\
\hline Expert 8 & 0.18 & 0.25 & 0.42 & 0.07 & 0.08 & 0.118 \\
\hline Mean & $\mathbf{0 . 2 8}$ & $\mathbf{0 . 2 1}$ & $\mathbf{0 . 2 8}$ & $\mathbf{0 . 0 8}$ & $\mathbf{0 . 1 5}$ & \\
\hline \multicolumn{2}{|l|}{ Disagreement } & & & & & 0.094 \\
\hline
\end{tabular}


Subgroup A has placed high importance on financial $(0.28)$ and organizational perspectives $(0.28)$ in for the purposes of assessment of adoption potential of wearable medical devices. The experts of this subgroup are closely tied with medical Informatics, management of information technologies in the hospital and high leadership/managerial executive roles in the clinic.

Table 36 Analysis of results in subgroup B of expert panel 1

\begin{tabular}{|c|c|c|c|c|c|c|}
\hline Panel 1 & Financial & Technical & Organizational & Patient & Interpersonal & Inconsistency \\
\hline Expert 1 & 0.04 & 0.23 & 0.11 & 0.33 & 0.28 & 0.029 \\
\hline Expert 2 & 0.11 & 0.13 & 0.14 & 0.49 & 0.13 & 0.035 \\
\hline Expert 3 & 0.21 & 0.29 & 0.1 & 0.33 & 0.08 & 0.024 \\
\hline Expert 4 & 0.28 & 0.4 & 0.05 & 0.22 & 0.06 & 0.018 \\
\hline Expert 7 & 0.16 & 0.15 & 0.15 & 0.37 & 0.19 & 0.004 \\
\hline Mean & 0.16 & 0.24 & 0.11 & 0.35 & 0.15 & \\
\hline \multicolumn{2}{|c|}{ Disagreement } & & & & & 0.089 \\
\hline
\end{tabular}

The results of the subgroup $B$ show that experts place higher importance on Patient perspective (0.35). Technical perspective gets the second place with the value of 0.24 . Financial, interpersonal and organizational perspectives are of the least importance to this group. The experts in this group are more closely tied to patient care and information technologies.

\subsection{Expert Panel 2}

\subsubsection{Expert panel 2 results}

Expert panel 2 evaluated the importance of the financial perspective criteria for the assessment of the technology adoption potential of wearable 
medical devices. Expert panel 2 consisted of 9 experts. The inconsistencies in expert opinions are below the value of 0.1 and the means of the relative importance of financial criteria of panel 2 are shown in Figure 25 below:

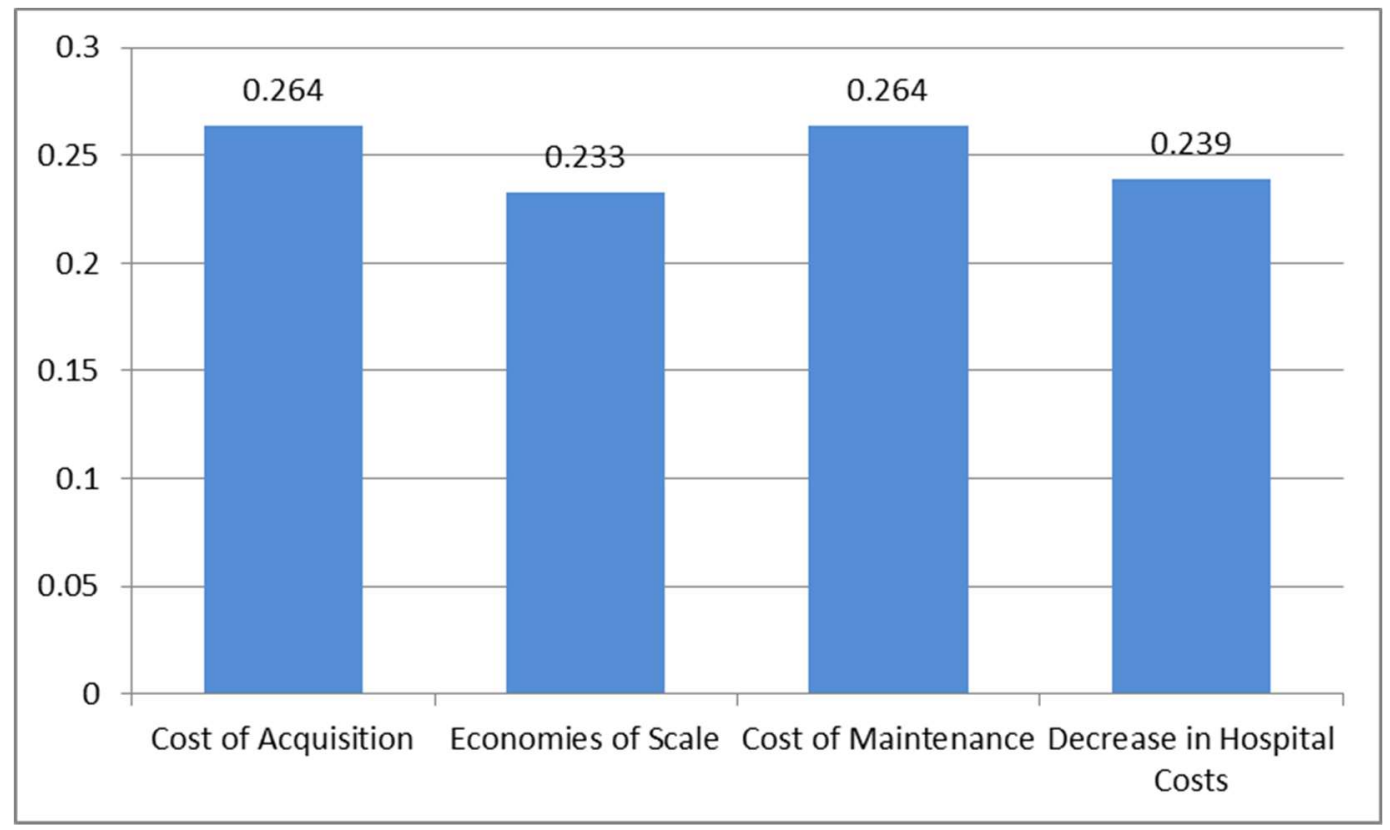

Figure 25 Relative importance of criteria in Financial perspective

According to the results, both cost of acquisition (26.4\%) and cost of maintenance $(26.4 \%)$ were considered the most important criteria of financial perspective. Savings from economies of scale (23.3\%) got the lowest score for importance.

\subsubsection{Analysis of expert panel 2 results}

The experts of panel 2 had inconsistencies within acceptable level. Table 37 with individual relative priority weights, aggregated group results, inconsistency levels and group disagreement is presented below. 
Table 37 Analysis of expert panel 2 results

\begin{tabular}{|c|c|c|c|c|c|}
\hline Panel 2 & $\begin{array}{l}\text { Cost of } \\
\text { Acquisition }\end{array}$ & $\begin{array}{l}\text { Economies } \\
\text { of Scale }\end{array}$ & $\begin{array}{l}\text { Cost of } \\
\text { Maintenance }\end{array}$ & $\begin{array}{l}\text { Decrease } \\
\text { in } \\
\text { Hospital } \\
\text { Costs }\end{array}$ & Inconsistency \\
\hline Expert 1 & 0.23 & 0.13 & 0.24 & 0.39 & 0.031 \\
\hline Expert 2 & 0.12 & 0.46 & 0.14 & 0.27 & 0.058 \\
\hline Expert 3 & 0.17 & 0.09 & 0.37 & 0.37 & 0.002 \\
\hline Expert 4 & 0.17 & 0.11 & 0.14 & 0.58 & 0.019 \\
\hline Expert 5 & 0.18 & 0.15 & 0.66 & 0.01 & 0.032 \\
\hline Expert 6 & 0.65 & 0.07 & 0.2 & 0.08 & 0.084 \\
\hline Expert 7 & 0.18 & 0.62 & 0.14 & 0.07 & 0.015 \\
\hline Expert 8 & 0.2 & 0.22 & 0.27 & 0.3 & 0.004 \\
\hline Expert 9 & 0.47 & 0.24 & 0.2 & 0.09 & 0.094 \\
\hline Mean & 0.264 & 0.233 & 0.264 & 0.240 & \\
\hline Disagreement & & & & & 0.18 \\
\hline
\end{tabular}

There was some disagreement (0.18) among the experts; therefore, hierarchical agglomerative clustering in $\mathrm{R}$ has been used to identify subgroups within expert panel 2. Four subgroups have been identified with hierarchical cluster analysis and the dendrogram is presented in Figure 26 below. 


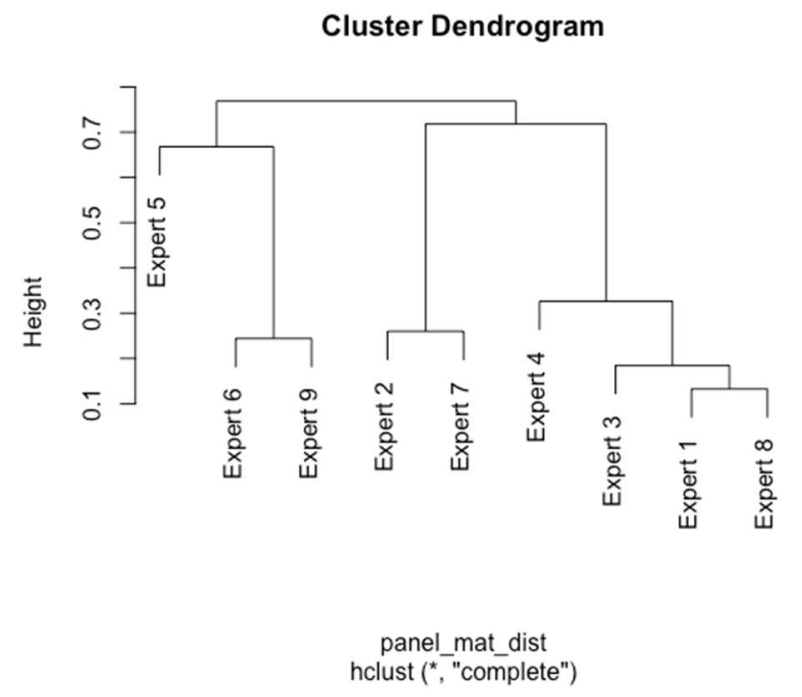

Figure 26 Dendrogram of subgroups of expert panel 2

Group disagreements of subgroup A (expert 5) and subgroup B (experts 6 and 9), subgroup $C$ (experts 2 and 7) and subgroup D (experts 1, 3, 4 and 8) are below the threshold value of 0.1 . The tables (Table 38, Table 39, Table 40 and Table 41) below show expert relative priorities, inconsistencies, aggregated group results and group disagreements for the two subgroups in panel 2: subgroup $A, B, C$ and $D$.

Table 38 Analysis of results in subgroup A of expert panel 2

\begin{tabular}{|c|c|c|c|c|c|}
\hline Panel 2 & $\begin{array}{l}\text { Cost of } \\
\text { Acquisition }\end{array}$ & $\begin{array}{l}\text { Economies } \\
\text { of Scale }\end{array}$ & $\begin{array}{l}\text { Cost of } \\
\text { Maintenance }\end{array}$ & $\begin{array}{l}\text { Decrease } \\
\text { in } \\
\text { Hospital } \\
\text { Costs }\end{array}$ & Inconsistency \\
\hline Expert 5 & 0.18 & 0.15 & 0.66 & 0.01 & 0.032 \\
\hline Mean & 0.18 & 0.15 & 0.66 & 0.01 & \\
\hline Disagreement & & & & & - \\
\hline
\end{tabular}


Subgroup A consists of one expert, who has placed high importance on cost of maintenance associated with adoption of wearable medical device (0.66), while considering the importance of decrease in hospital costs as negligible. The expert holds a high executive position in the hospital.

Table 39 Analysis of results in subgroup B of expert panel 2

\begin{tabular}{|c|c|c|c|c|c|}
\hline Panel 2 & $\begin{array}{l}\text { Cost of } \\
\text { Acquisition }\end{array}$ & $\begin{array}{l}\text { Economies } \\
\text { of Scale }\end{array}$ & $\begin{array}{l}\text { Cost of } \\
\text { Maintenance }\end{array}$ & $\begin{array}{l}\text { Decrease } \\
\text { in } \\
\text { Hospital } \\
\text { Costs }\end{array}$ & Inconsistency \\
\hline Expert 6 & 0.65 & 0.07 & 0.2 & 0.08 & 0.084 \\
\hline Expert 9 & 0.47 & 0.24 & 0.2 & 0.09 & 0.094 \\
\hline Mean & 0.56 & 0.15 & 0.20 & 0.09 & \\
\hline Disagreement & & & & & 0.086 \\
\hline
\end{tabular}

Subgroup B consists of two experts, who have placed high importance on cost of acquisition associated with adoption of wearable medical device (0.56), followed by cost of maintenance (0.20) and economies of scale (0.15), while considering the importance of decrease in hospital costs a minor criteria (0.09).

Table 40 Analysis of results in subgroup $C$ of expert panel 2

\begin{tabular}{|l|r|r|r|r|r|}
\hline & Cost of \\
Panel 2 & Acquisition & $\begin{array}{l}\text { Economies } \\
\text { of Scale }\end{array}$ & $\begin{array}{l}\text { Cost of } \\
\text { Maintenance }\end{array}$ & $\begin{array}{l}\text { Decrease } \\
\text { in } \\
\text { Hospital } \\
\text { Costs }\end{array}$ & Inconsistency \\
\hline Expert 2 & 0.12 & 0.46 & 0.14 & 0.27 & 0.058 \\
\hline Expert 7 & 0.18 & 0.62 & 0.14 & 0.07 & 0.015 \\
\hline Mean & $\mathbf{0 . 1 5}$ & $\mathbf{0 . 5 4}$ & $\mathbf{0 . 1 4}$ & $\mathbf{0 . 1 7}$ & \\
\hline Disagreement & & & & & 0.092 \\
\hline
\end{tabular}

Two experts of subgroup $C$ consider economies of scale ( 0.54$)$ of the highest importance towards wearable technology adoption, distantly followed by 
cost of acquisition (0.15), decrease in hospital costs (0.17) and cost of maintenance (0.14). The disagreement in this group is within the acceptable level.

Table 41 Analysis of results in subgroup D of expert panel 2

\begin{tabular}{|c|c|c|c|c|c|}
\hline Panel 2 & $\begin{array}{l}\text { Cost of } \\
\text { Acquisition }\end{array}$ & $\begin{array}{l}\text { Economies } \\
\text { of Scale }\end{array}$ & $\begin{array}{l}\text { Cost of } \\
\text { Maintenance }\end{array}$ & $\begin{array}{l}\text { Decrease } \\
\text { in } \\
\text { Hospital } \\
\text { Costs }\end{array}$ & Inconsistency \\
\hline Expert 1 & 0.23 & 0.13 & 0.24 & 0.39 & 0.031 \\
\hline Expert 3 & 0.17 & 0.09 & 0.37 & 0.37 & 0.002 \\
\hline Expert 4 & 0.17 & 0.11 & 0.14 & 0.58 & 0.019 \\
\hline Expert 8 & 0.2 & 0.22 & 0.27 & 0.3 & 0.004 \\
\hline Mean & 0.19 & 0.14 & 0.26 & 0.41 & \\
\hline Disagreement & & & & & 0.083 \\
\hline
\end{tabular}

Subgroup $D$ is the largest subgroup of panel 2 and it consists of four experts. The experts place decrease of hospital costs as the most important financial criteria (0.41). Cost of maintenance is the second important financial criteria according to the subgroup $D(0.26)$, followed by cost of acquisition (0.19) and economies of scale $(0.14)$.

\subsection{Expert Panel 3}

\subsubsection{Expert panel 3 results}

Experts in expert panel 3 were asked to evaluate relative importance of technical criteria with respect to overall mission of assessment of technology adoption potential of medical devices for a case of wearable sensor products for 
pervasive spine care in neurosurgery and orthopedics. The arithmetic means of the relative importance of technical criteria of panel 3 [10 expert opinions] are shown in Figure 27 below:

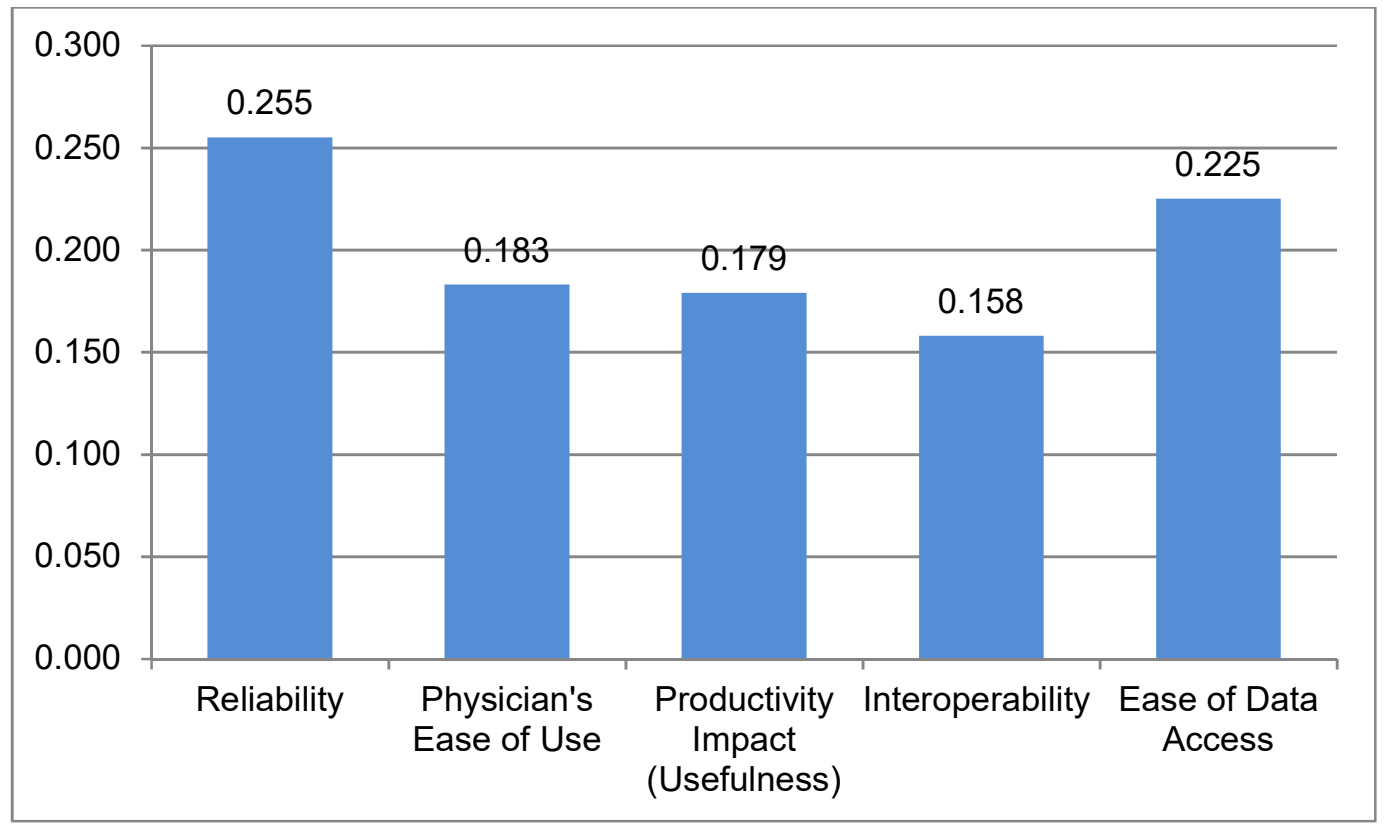

Figure 27 Relative importance of criteria in Technical perspective

According to the expert opinions of expert panel 3 , reliability is the highest ranked criterion (25.5\%) out of technical perspective criteria. Ease of data access is the second highly regarded criteria with $22.5 \%$ relative weight. Physician's ease of use, usefulness and interoperability have the lowest relative weights of importance of $18.3 \%, 17.9 \%$ and $15.8 \%$ respectively.

\subsubsection{Analysis of expert panel 3 results}

Two experts in expert panel 3 had inconsistency levels above 0.1 and their Root Sum Variance was calculated according to (Abbas 2016). The 
threshold of acceptable inconsistency with RSV value for 5 decision variables at $\alpha=0.05$ is 0.2651 RSV values for the two experts with inconsistencies $(0.140$ and 0.118 ) were 0.312 and 0.2648 respectively. Since RSV value of 0.2648 is below the threshold of 0.2651 , the evaluations of that expert were included in the analysis. There was also some disagreement among the experts $(0.106)$ present and will be discussed in this chapter. The table with individual relative priority weights, aggregated group results, inconsistency levels and group disagreement, is presented below Table 42 .

Table 42 Analysis of expert panel 3 results

\begin{tabular}{|c|c|c|c|c|c|c|}
\hline Panel 3 & Reliability & $\begin{array}{l}\text { Physician's } \\
\text { Ease of Use }\end{array}$ & $\begin{array}{l}\text { Productivity } \\
\text { Impact } \\
\text { (Usefulness) }\end{array}$ & Interoperability & $\begin{array}{l}\text { Ease of } \\
\text { Data } \\
\text { Access } \\
\end{array}$ & Inconsistency \\
\hline Expert 1 & 0.26 & 0.1 & 0.27 & 0.13 & 0.23 & 0.064 \\
\hline Expert 2 & 0.25 & 0.27 & 0.28 & 0.07 & 0.13 & 0.009 \\
\hline Expert 3 & 0.33 & 0.22 & 0.22 & 0.12 & 0.11 & 0.029 \\
\hline Expert 4 & 0.60 & 0.05 & 0.15 & 0.03 & 0.18 & 0.118 \\
\hline Expert 5 & 0.07 & 0.24 & 0.06 & 0.32 & 0.31 & 0.098 \\
\hline Expert 6 & 0.19 & 0.22 & 0.18 & 0.17 & 0.24 & 0.025 \\
\hline Expert 7 & 0.39 & 0.13 & 0.27 & 0.12 & 0.09 & 0.018 \\
\hline Expert 8 & 0.23 & 0.16 & 0.09 & 0.23 & 0.29 & 0.014 \\
\hline Expert 9 & 0.09 & 0.12 & 0.2 & 0.27 & 0.33 & 0.032 \\
\hline Expert 10 & 0.15 & 0.34 & 0.09 & 0.09 & 0.34 & 0.002 \\
\hline Mean & 0.255 & 0.183 & 0.179 & 0.158 & 0.225 & \\
\hline \multicolumn{2}{|c|}{ Disagreement } & & & & & 0.106 \\
\hline
\end{tabular}

There was slight disagreement (0.106) among the experts. Using hierarchical agglomerative clustering in $\mathrm{R}$, two subgroups were identified within expert panel 3. The dendrogram of hierarchical clustering is presented in Figure 28. 


\section{Cluster Dendrogram}

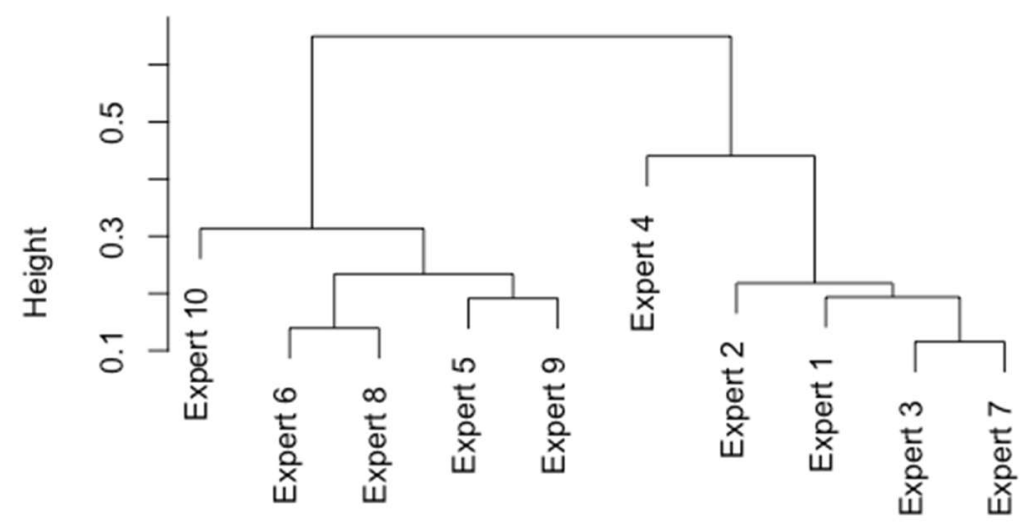

\footnotetext{
panel_mat_dist hclust ( ${ }^{*}$ " "complete")
}

Figure 28 Dendrogram of subgroups of expert panel 3

Subgroup A for expert panel 3 consists of five experts (expert 5, 6, 8, 9 and 10 ) and has overall disagreement value of 0.071 . Subgroup $B$ also consists of five experts (experts $1,2,3,4$ and 7 ), has disagreement value of 0.085 . The tables below (Table 43 and Table 44) show expert relative priorities, inconsistencies, aggregated group results and group disagreements for the two subgroups in panel 3: subgroup A and B. 
Table 43 Analysis of results in subgroup A of expert panel 3

\begin{tabular}{|c|c|c|c|c|c|c|}
\hline Panel 3 & Reliability & $\begin{array}{l}\text { Physician's } \\
\text { Ease of Use }\end{array}$ & $\begin{array}{l}\text { Productivity } \\
\text { Impact } \\
\text { (Usefulness) }\end{array}$ & Interoperability & $\begin{array}{l}\text { Ease of } \\
\text { Data } \\
\text { Access } \\
\end{array}$ & Inconsistency \\
\hline Expert 5 & 0.07 & 0.24 & 0.06 & 0.32 & 0.31 & 0.098 \\
\hline Expert 6 & 0.19 & 0.22 & 0.18 & 0.17 & 0.24 & 0.025 \\
\hline Expert 8 & 0.23 & 0.16 & 0.09 & 0.23 & 0.29 & 0.014 \\
\hline Expert 9 & 0.09 & 0.12 & 0.2 & 0.27 & 0.33 & 0.032 \\
\hline Expert 10 & 0.15 & 0.34 & 0.09 & 0.09 & 0.34 & 0.002 \\
\hline Mean & 0.15 & 0.21 & 0.12 & 0.22 & 0.30 & \\
\hline \multicolumn{2}{|c|}{ Disagreement } & & & & & 0.071 \\
\hline
\end{tabular}

Experts of subgroup A of expert panel 3 overall consider ease of data access as the most important technical criteria $(0.30)$, followed by interoperability $(0.22)$ and physician's ease of use (0.21). The disagreement is within the acceptable level in this group.

Table 44 Analysis of results in subgroup B of expert panel 3

\begin{tabular}{|c|c|c|c|c|c|c|}
\hline Panel 3 & Reliability & $\begin{array}{l}\text { Physician's } \\
\text { Ease of Use }\end{array}$ & $\begin{array}{l}\text { Productivity } \\
\text { Impact } \\
\text { (Usefulness) }\end{array}$ & Interoperability & $\begin{array}{l}\text { Ease of } \\
\text { Data } \\
\text { Access }\end{array}$ & Inconsistency \\
\hline Expert 1 & 0.26 & 0.1 & 0.27 & 0.13 & 0.23 & 0.064 \\
\hline Expert 2 & 0.25 & 0.27 & 0.28 & 0.07 & 0.13 & 0.009 \\
\hline Expert 3 & 0.33 & 0.22 & 0.22 & 0.12 & 0.11 & 0.029 \\
\hline Expert 4 & 0.60 & 0.05 & 0.15 & 0.03 & 0.18 & 0.118 \\
\hline Expert 7 & 0.39 & 0.13 & 0.27 & 0.12 & 0.09 & 0.018 \\
\hline Mean & 0.36 & 0.15 & 0.24 & 0.10 & 0.15 & \\
\hline \multicolumn{2}{|c|}{ Disagreement } & & & & & 0.085 \\
\hline
\end{tabular}

Subgroup B of expert panel 3 places reliability as the most important technical criteria as related to the mission (0.36), while productivity impact (usefulness) gets a second place with 0.24 . Interoperability $(0.10)$ is the lowest important technical criteria according to the group's results. 


\subsection{Expert Panel 4}

\subsubsection{Expert panel 4 results}

Experts in expert panel 4 were evaluating relative importance of criteria under organizational perspective with respect to overall mission of assessment of technology adoption potential of medical devices for a case of wearable sensor products for pervasive spine care in neurosurgery and orthopedics. The arithmetic means of the relative importance of organizational criteria of panel 4 [9 expert opinions] are shown in Figure 29 below:

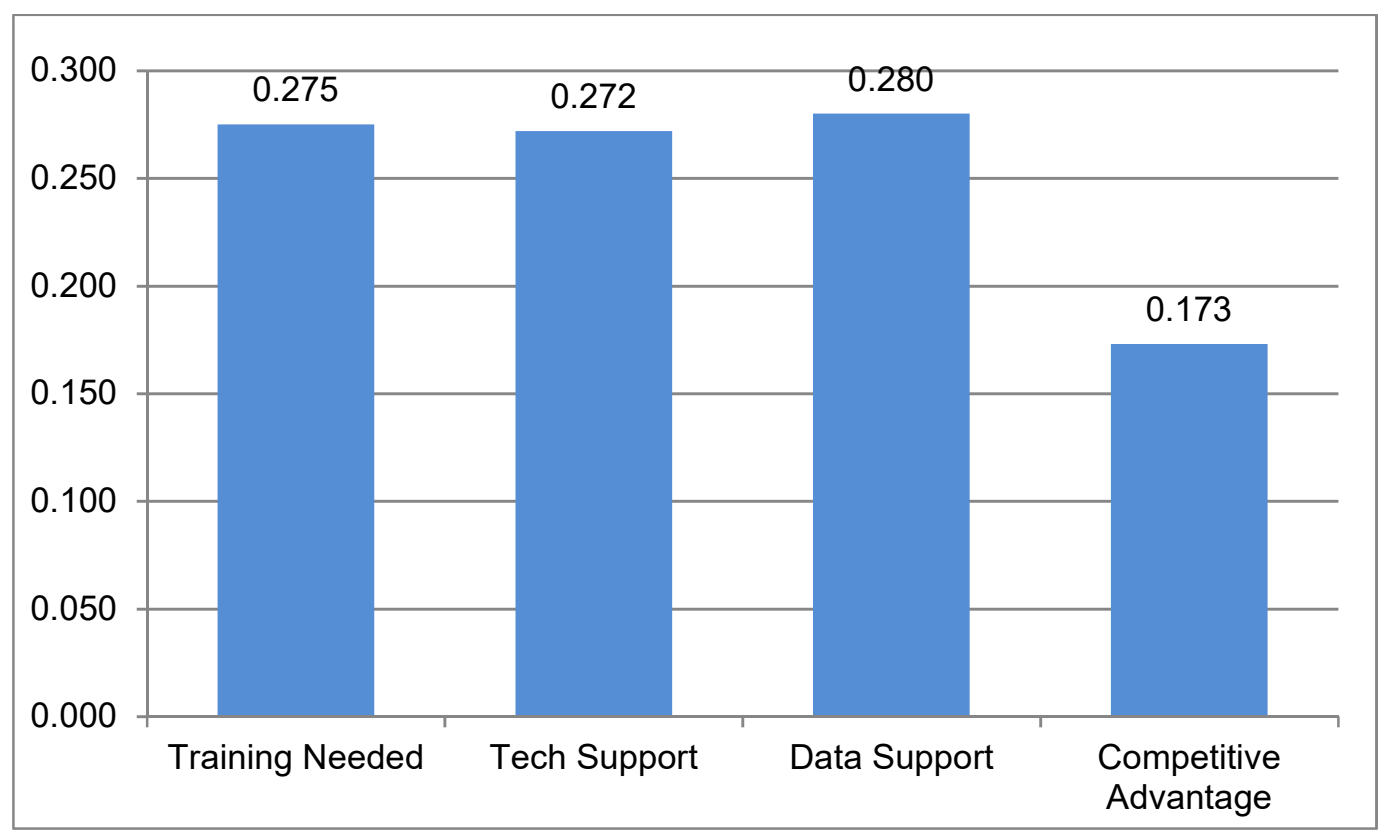

Figure 29 Relative importance of criteria in Organizational perspective

According to results of expert panel 4 , data support is the highest valued perspective in organizational perspective (28\%), although it's very closely 
followed by 'training needed' (27.5\%) and 'tech support' (27.2\%). Competitive advantage has the lowest importance (17.3\%) according to experts in panel 4.

\subsubsection{Analysis of expert panel 4 results}

All experts of panel 4 had inconsistencies within acceptable level. Some disagreement among the experts (0.106) was present and will be discussed in the analysis of expert panel 4 section. Table 45 with individual relative priority weights, aggregated group results, inconsistency levels and group disagreement, is shown below.

Table 45 Analysis of results of expert panel 4

\begin{tabular}{|l|r|r|r|r|r|}
\hline & $\begin{array}{l}\text { Training } \\
\text { Needed }\end{array}$ & $\begin{array}{l}\text { Tech } \\
\text { Support }\end{array}$ & Data Support & $\begin{array}{l}\text { Competitive } \\
\text { Advantage }\end{array}$ & Inconsistency \\
\hline Expert 1 & 0.38 & 0.21 & 0.22 & 0.19 & 0.046 \\
\hline Expert 2 & 0.09 & 0.31 & 0.46 & 0.14 & 0.068 \\
\hline Expert 3 & 0.11 & 0.26 & 0.32 & 0.31 & 0.011 \\
\hline Expert 4 & 0.55 & 0.25 & 0.11 & 0.09 & 0.073 \\
\hline Expert 5 & 0.28 & 0.2 & 0.27 & 0.25 & 0.011 \\
\hline Expert 6 & 0.22 & 0.34 & 0.2 & 0.24 & 0.035 \\
\hline Expert 7 & 0.3 & 0.27 & 0.27 & 0.16 & 0.005 \\
\hline Expert 8 & 0.39 & 0.22 & 0.26 & 0.13 & 0.018 \\
\hline Expert 9 & 0.17 & 0.39 & 0.4 & 0.04 & 0.000 \\
\hline Mean & $\mathbf{0 . 2 7 5}$ & $\mathbf{0 . 2 7 2}$ & $\mathbf{0 . 2 8 0}$ & $\mathbf{0 . 1 7 3}$ & \\
\hline Disagreement & & & & & 0.106 \\
\hline
\end{tabular}

Hierarchical agglomerative clustering in $\mathrm{R}$ was used and two subgroups were identified within expert panel 3 . The dendrogram of hierarchical clustering is presented in Figure 30. 


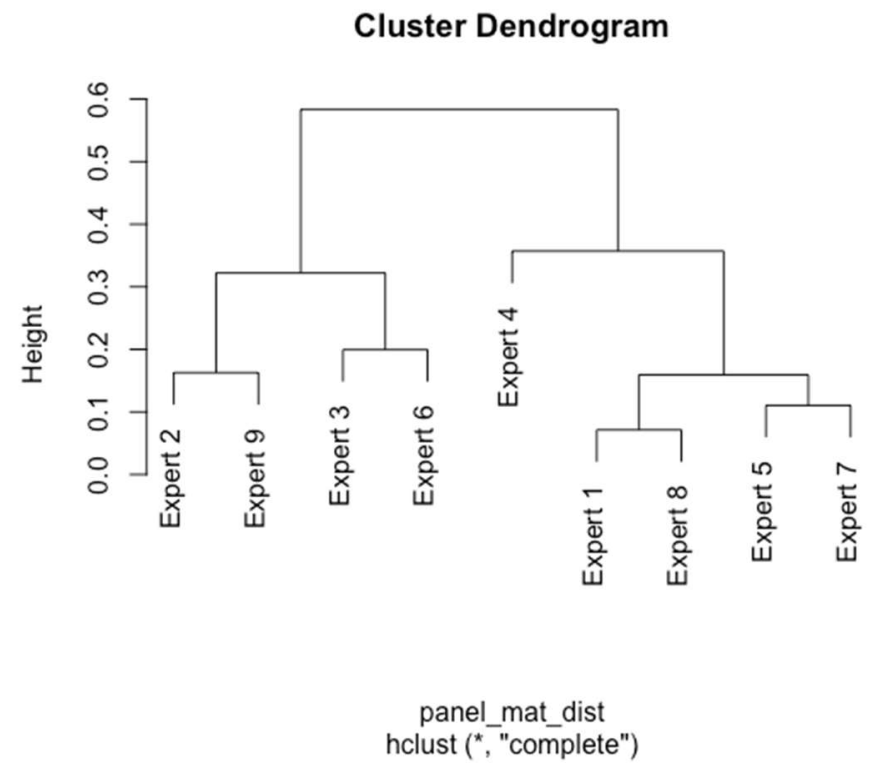

Figure 30 Dendrogram of subgroups of expert panel 4

Subgroup A for expert panel 4 consists of four experts (expert 2, 3, 6 and 9) and has overall disagreement value of 0.092. Subgroup B is the largest one and consists of five experts (experts 1, 4, 5, 7 and 8) and has disagreement value of 0.071 . The tables (Table 46 and Table 47 ) below show expert relative priorities, inconsistencies, aggregated group results and group disagreements for the two subgroups in panel 4: subgroup A and B.

Table 46 Analysis of results in subgroup A of expert panel 4

\begin{tabular}{|l|r|r|r|r|r|}
\hline Panel 4 & $\begin{array}{r}\text { Training } \\
\text { Needed }\end{array}$ & $\begin{array}{l}\text { Tech } \\
\text { Support }\end{array}$ & Data Support & $\begin{array}{l}\text { Competitive } \\
\text { Advantage }\end{array}$ & Inconsistency \\
\hline Expert 2 & 0.09 & 0.31 & 0.46 & 0.14 & 0.068 \\
\hline Expert 3 & 0.11 & 0.26 & 0.32 & 0.31 & 0.011 \\
\hline Expert 6 & 0.22 & 0.34 & 0.2 & 0.24 & 0.035 \\
\hline Expert 9 & 0.17 & 0.39 & 0.4 & 0.04 & 0.000 \\
\hline Mean & $\mathbf{0 . 1 5}$ & $\mathbf{0 . 3 2}$ & $\mathbf{0 . 3 5}$ & $\mathbf{0 . 1 8}$ & \\
\hline Disagreement & & & & & 0.092 \\
\hline
\end{tabular}


Experts in subgroup A of panel 4 indicated that data support is the most important criterion in organizational perspective (0.35). Tech support is a close second criterion (0.32), while 'competitive advantage' and 'training needed' are distant third and fourth important criteria with values of 0.18 and 0.15 respectively.

Table 47 Analysis of results in subgroup B of expert panel 4

\begin{tabular}{|l|r|r|r|r|r|}
\hline & $\begin{array}{l}\text { Training } \\
\text { Needed }\end{array}$ & $\begin{array}{l}\text { Tech } \\
\text { Support }\end{array}$ & Data Support & $\begin{array}{l}\text { Competitive } \\
\text { Advantage }\end{array}$ & Inconsistency \\
\hline Expert 1 & 0.38 & 0.21 & 0.22 & 0.19 & 0.046 \\
\hline Expert 4 & 0.55 & 0.25 & 0.11 & 0.09 & 0.073 \\
\hline Expert 5 & 0.28 & 0.2 & 0.27 & 0.25 & 0.011 \\
\hline Expert 7 & 0.3 & 0.27 & 0.27 & 0.16 & 0.005 \\
\hline Expert 8 & 0.39 & 0.22 & 0.26 & 0.13 & 0.018 \\
\hline Mean & $\mathbf{0 . 3 8}$ & $\mathbf{0 . 2 3}$ & $\mathbf{0 . 2 3}$ & $\mathbf{0 . 1 6}$ & \\
\hline Disagreement & & & & & 0.071 \\
\hline
\end{tabular}

Subgroup B of expert panel 4 placed 'training needed' as the most important organizational criteria (0.38). Competitive advantage (0.16) was the least important criteria in this group. Both tech support and data support were equally important with the value of 0.23 .

\subsection{Expert Panel 5}

\subsubsection{Expert panel 5 results}

Relative importance of patient criteria was the evaluation objective of expert panel 5. Experts of this panel evaluated patient criteria with respect to overall mission of assessment of technology adoption potential of medical 
devices for a case of wearable sensor products for pervasive spine care in neurosurgery and orthopedics. The arithmetic means of the relative importance of patient criteria of panel 5 [7 expert opinions] are shown in Figure 31 below:

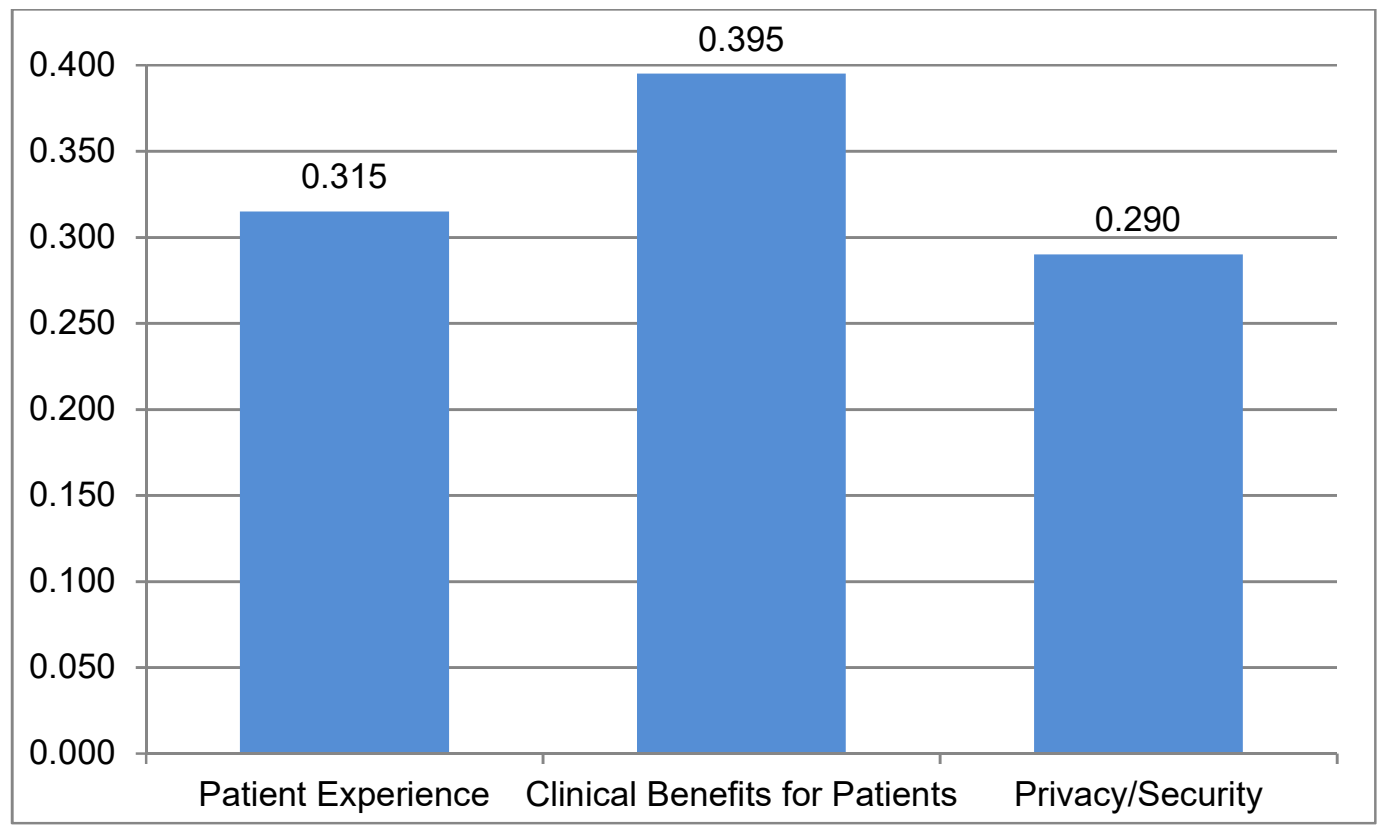

Figure 31 Relative importance of criteria in Patient perspective

The most important criteria in patient perspective as related to the overall mission and according to the experts of panel 5 , is criterion of clinical benefits for patients (39.5\%). The second place holds criterion of patient experience (31.5\%). Privacy and security has the lowest priority in this group of criteria according to the experts with $29 \%$.

\subsubsection{Analysis of expert panel 5 results}

One expert in expert panel 5 had inconsistency level above 0.1 and this expert's Root Sum Variance was calculated according to (Abbas 2016). The 
threshold of acceptable inconsistency with RSV value for 3 decision variables at $\alpha=0.05$ is 0.0014 . RSV value for this expert with inconsistency $(0.162)$ was 0.28105 . Since RSV value of 0.28105 is above the threshold of 0.0 .0014 , the evaluations of this expert were not included in the analysis. All other 7 experts of panel 5 were consistent in their evaluations and had inconsistencies values within acceptable level. There was a slight disagreement (0.108) within the panel, which was analyzed with hierarchical cluster analysis. The table with individual relative priority weights, aggregated group results, inconsistency levels and group disagreement is shown below (Table 48).

Table 48 Analysis of results of expert panel 5

\begin{tabular}{|c|c|c|c|c|}
\hline Panel 5 & $\begin{array}{l}\text { Patient } \\
\text { Experience }\end{array}$ & $\begin{array}{l}\text { Clinical Benefits } \\
\text { for Patients }\end{array}$ & Privacy/Security & Inconsistency \\
\hline Expert 1 & 0.31 & 0.47 & 0.23 & 0.003 \\
\hline Expert 2 & 0.24 & 0.32 & 0.43 & 0.023 \\
\hline Expert 3 & 0.29 & 0.29 & 0.43 & 0.000 \\
\hline Expert 4 & 0.34 & 0.33 & 0.33 & 0.000 \\
\hline Expert 5 & 0.33 & 0.33 & 0.33 & 0.000 \\
\hline Expert 6 & 0.22 & 0.55 & 0.23 & 0.056 \\
\hline Expert 7 & 0.48 & 0.48 & 0.05 & 0.000 \\
\hline Mean & 0.31 & 0.39 & 0.29 & \\
\hline Disagreement & & & & 0.108 \\
\hline
\end{tabular}

Hierarchical agglomerative clustering in $\mathrm{R}$ has been used to identify subgroups within expert panel 5 to analyze the disagreement. Three subgroups have been identified with hierarchical cluster analysis and the dendrogram is presented in Figure 32 below. 


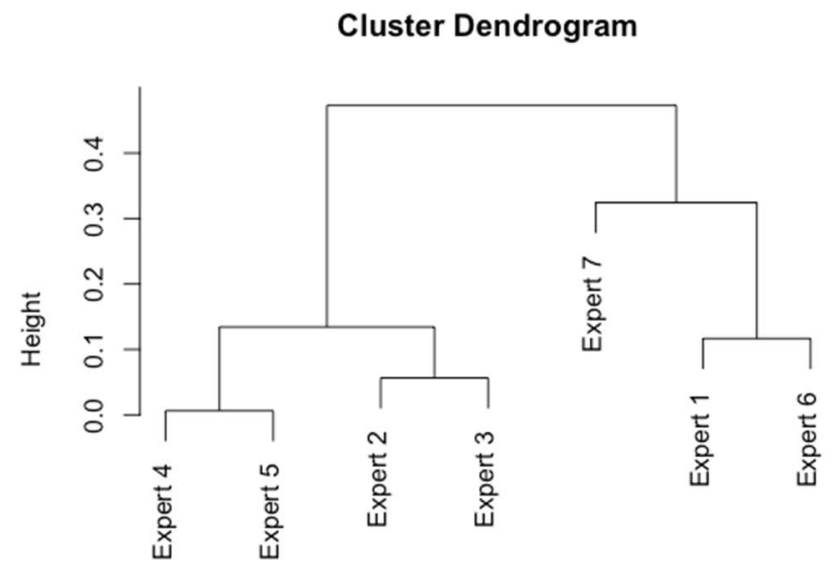

panel_mat_dist
hclust ${ }^{\star}$, "complete")

Figure 32 Dendrogram of subgroups of expert panel 5

Four experts (expert 2, 3, 4 and 5) compose subgroup A of expert panel 5. It is also the largest group and has overall disagreement value of 0.043 .

Subgroup B consists of two experts (expert 1and 6) and has disagreement value of 0.048 . Subgroup $C$ consists of one expert (expert 7). The tables below (Table 49, Table 50 and Table 51) show expert relative priorities, inconsistencies, aggregated group results and group disagreements for the three subgroups in panel 5: subgroup A, B and C. 
Table 49 Analysis of results in subgroup A of expert panel 5

\begin{tabular}{|c|c|c|c|c|}
\hline Panel 5 & $\begin{array}{l}\text { Patient } \\
\text { Experience }\end{array}$ & $\begin{array}{l}\text { Clinical Benefits } \\
\text { for Patients }\end{array}$ & Privacy/Security & Inconsistency \\
\hline Expert 2 & 0.24 & 0.32 & 0.43 & 0.023 \\
\hline Expert 3 & 0.29 & 0.29 & 0.43 & 0.000 \\
\hline Expert 4 & 0.34 & 0.33 & 0.33 & 0.000 \\
\hline Expert 5 & 0.33 & 0.33 & 0.33 & 0.000 \\
\hline Mean & 0.30 & 0.32 & 0.38 & \\
\hline Disagreement & & & & 0.043 \\
\hline
\end{tabular}

Experts in subgroup A of panel 5 placed privacy and security as the most important criteria in patient perspective (0.38). 'Clinical benefits to patients' was the second important criterion (0.32) and patient experience took the third place (0.30) according to subgroup A of panel 5.

Table 50 Analysis of results in subgroup B of expert panel 5

\begin{tabular}{|c|c|c|c|c|}
\hline Panel 5 & $\begin{array}{l}\text { Patient } \\
\text { Experience }\end{array}$ & $\begin{array}{l}\text { Clinical Benefits } \\
\text { for Patients }\end{array}$ & Privacy/Security & Inconsistency \\
\hline Expert 1 & 0.31 & 0.47 & 0.23 & 0.003 \\
\hline Expert 6 & 0.22 & 0.55 & 0.23 & 0.056 \\
\hline Mean & 0.26 & 0.51 & 0.23 & \\
\hline Disagreement & & & & 0.048 \\
\hline
\end{tabular}

Subgroup B of expert panel 4 placed 'clinical benefits to patient' as the most important patient criteria (0.51). Patient experience was regarded as the second important criteria in patient perspective $(0.26)$, followed by privacy/security (0.23). 
Table 51 Analysis of results in subgroup $\mathrm{C}$ of expert panel 5

\begin{tabular}{|c|c|c|c|c|}
\hline Panel 5 & $\begin{array}{l}\text { Patient } \\
\text { Experience }\end{array}$ & $\begin{array}{l}\text { Clinical Benefits } \\
\text { for Patients }\end{array}$ & Privacy/Security & Inconsistency \\
\hline Expert 7 & 0.48 & 0.48 & 0.05 & 0.000 \\
\hline Mean & 0.48 & 0.48 & 0.05 & \\
\hline Disagreement & & & & 0.000 \\
\hline
\end{tabular}

Subgroup $\mathrm{C}$ of panel 5 has one expert, who gave equally high importance to 'clinical benefits for patients' and 'patient experience', but considered privacy and security as criteria of low importance (0.05) relative to the mission.

\subsection{Expert Panel 6}

\subsubsection{Expert panel 6 results}

Experts in expert panel 6 were evaluating relative importance of criteria under interpersonal perspective with respect to overall mission of assessment of technology adoption potential of medical devices for a case of wearable sensor products for pervasive spine care in neurosurgery and orthopedics. The arithmetic means of the relative importance of interpersonal criteria of panel 6 [11 expert opinions] are shown in Figure 33 below: 


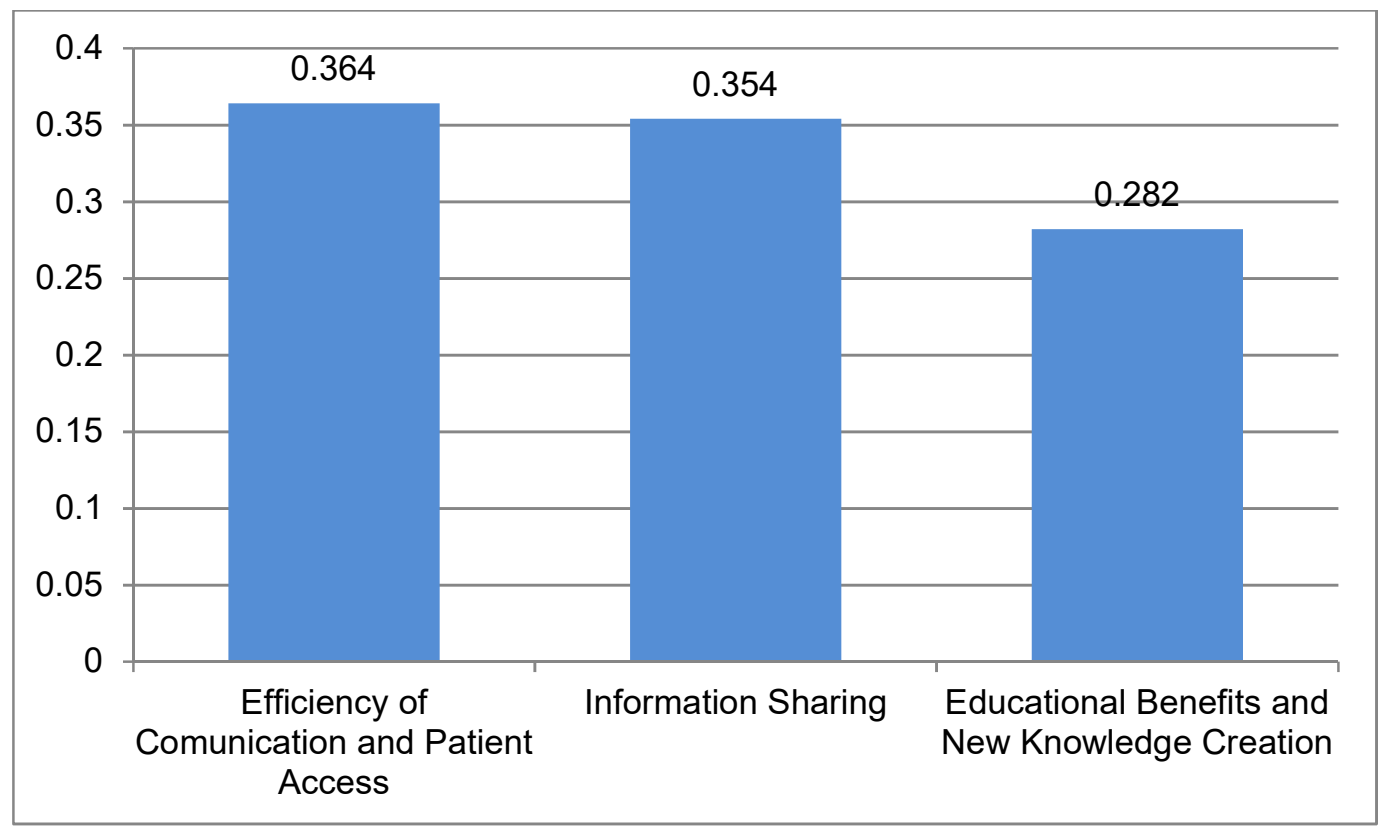

Figure 33 Relative importance of criteria in Interpersonal perspective

According to results of expert panel 6, 'efficiency of communication and patient access' is the highest valued criterion in interpersonal perspective (36.4\%), however, it's very closely followed by 'information sharing' (35.4\%). 'Educational benefits and new knowledge creation' has the lowest importance (28.2\%) according to experts in panel 6.

\subsubsection{Analysis of expert panel 6 results}

All 11 experts of panel 5 were consistent in their evaluations and had inconsistencies values within acceptable level. There was some disagreement (0.138) within the panel, which was analyzed with hierarchical cluster analysis. Table 52 with individual relative priority weights, aggregated group results, inconsistency levels and group disagreement is shown below. 
Table 52 Analysis of results of expert panel 6

\begin{tabular}{|c|c|c|c|c|}
\hline Panel 6 & $\begin{array}{l}\text { Efficiency of } \\
\text { Communication } \\
\text { and Patient Access }\end{array}$ & $\begin{array}{l}\text { Information } \\
\text { Sharing }\end{array}$ & $\begin{array}{l}\text { Educational Benefits } \\
\text { and New Knowledge } \\
\text { Creation }\end{array}$ & Inconsistency \\
\hline Expert 1 & 0.35 & 0.33 & 0.32 & 0.000 \\
\hline Expert 2 & 0.58 & 0.27 & 0.15 & 0.029 \\
\hline Expert 3 & 0.33 & 0.33 & 0.33 & 0.000 \\
\hline Expert 4 & 0.49 & 0.28 & 0.23 & 0.008 \\
\hline Expert 5 & 0.66 & 0.28 & 0.07 & 0.000 \\
\hline Expert 6 & 0.33 & 0.33 & 0.33 & 0.000 \\
\hline Expert 7 & 0.33 & 0.33 & 0.33 & 0.000 \\
\hline Expert 8 & 0.17 & 0.47 & 0.36 & 0.004 \\
\hline Expert 9 & 0.43 & 0.29 & 0.29 & 0.000 \\
\hline Expert 10 & 0.17 & 0.3 & 0.53 & 0.022 \\
\hline Expert 11 & 0.16 & 0.67 & 0.16 & 0.000 \\
\hline Mean & 0.36 & 0.35 & 0.28 & \\
\hline Disagreement & & & & 0.138 \\
\hline
\end{tabular}

Hierarchical agglomerative clustering in $\mathrm{R}$ has been used to identify subgroups within expert panel 6 to analyze the disagreement. As shown in table above, the disagreement value was 0.138 . Four subgroups have been identified with hierarchical cluster analysis and the dendrogram is presented in Figure 34. 


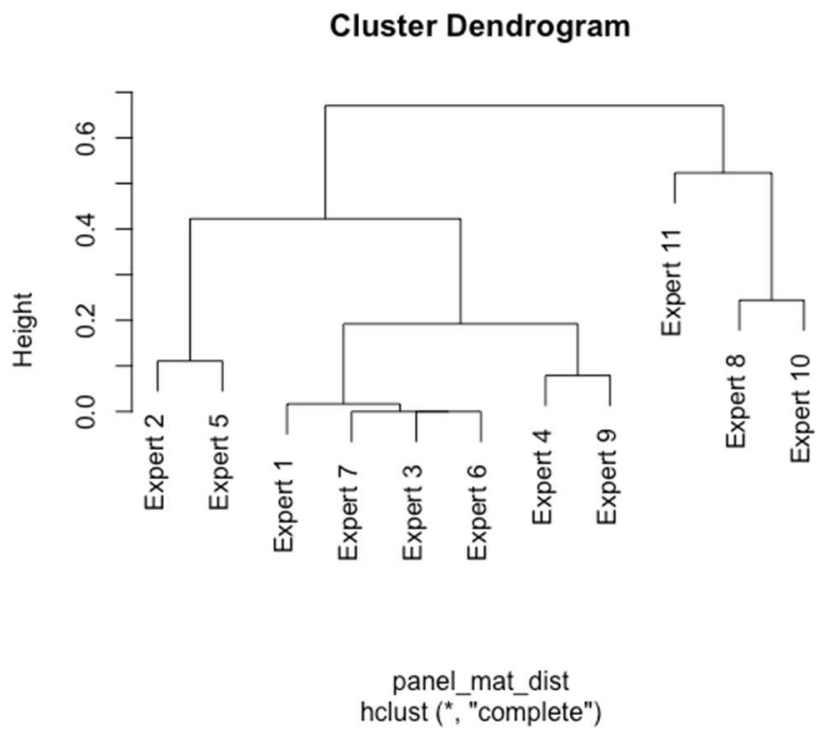

Figure 34 Dendrogram of subgroups of expert panel 6

Eight experts (experts 1-7 and 9) compose subgroup A of expert panel 6. It is also the largest group and has overall disagreement value of 0.095 .

Subgroup B, C and D consist of one expert each: expert 8, 10 and 11 respectively since their relative importance values were different. Expert 8 (subgroup B) placed the highest value on information sharing $(0.47)$, followed by 'educational benefits and new knowledge creation' (0.36), while 'efficiency of communication' was third (0.17). Expert 10 (subgroup C) felt very strongly about 'educational benefits' criteria ( 0.53$)$, followed by 'information sharing' $(0.30)$ and placed 'efficiency of communication' third (0.17). Expert 11 (subgroup D) evaluated interpersonal criteria with the highest importance value for 'information sharing' (0.67), and equal importance (0.16) for 'efficiency of communication' and 'educational benefits' criteria. The common theme in evaluations given by experts 8,10 and 11 is their evaluation of 'efficiency of communication and 
patient access' criterion, since this group of experts placed it third with values of 0.17 and 0.16 , while subgroup $A$ of panel 6 had different views. It should be noted that the views of those three experts didn't shift the aggregate results of the panel, which placed 'efficiency of communication and patient access' as the most important criteria. Table 53 provides the view of expert relative priorities, inconsistencies, aggregated group results and group disagreements for the largest subgroups in panel 6: subgroup A.

Table 53 Analysis of results in subgroup A of expert panel 6

\begin{tabular}{|c|c|c|c|c|}
\hline Panel 6 & $\begin{array}{l}\text { Efficiency of } \\
\text { Communication } \\
\text { and Patient Access }\end{array}$ & $\begin{array}{l}\text { Information } \\
\text { Sharing }\end{array}$ & $\begin{array}{l}\text { Educational Benefits } \\
\text { and New Knowledge } \\
\text { Creation }\end{array}$ & Inconsistency \\
\hline Expert 1 & 0.35 & 0.33 & 0.32 & 0.000 \\
\hline Expert 2 & 0.58 & 0.27 & 0.15 & 0.029 \\
\hline Expert 3 & 0.33 & 0.33 & 0.33 & 0.000 \\
\hline Expert 4 & 0.49 & 0.28 & 0.23 & 0.008 \\
\hline Expert 5 & 0.66 & 0.28 & 0.07 & 0.000 \\
\hline Expert 6 & 0.33 & 0.33 & 0.33 & 0.000 \\
\hline Expert 7 & 0.33 & 0.33 & 0.33 & 0.000 \\
\hline Expert 9 & 0.43 & 0.29 & 0.29 & 0.000 \\
\hline Mean & 0.44 & 0.31 & 0.26 & \\
\hline Disagreement & & & & 0.095 \\
\hline
\end{tabular}

Experts in subgroup A of panel 5 placed 'efficiency of communication and patient access' as the most important criteria in interpersonal perspective (0.44). 'Information sharing' was the second most important criteria (0.31) and 'educational benefits and new knowledge creation' took the third place $(0.26)$ according to subgroup A of panel 6 . 


\subsection{Expert Panel 7}

Experts of expert panel 7 compared model alternatives - wearable medical devices with respect to each criterion. Expert panel 7 consisted of 8 experts; however, since experts were assigned to evaluate model alternatives according to their expertise, not all of the experts provided comparisons for all criteria. Model alternatives were evaluated with respect to 19 criteria.

\subsubsection{Expert panel 7.1 results}

Experts of expert panel 7.1 analyzed financial criteria. The inconsistencies of the expert opinions were within acceptable level. The arithmetic means of the relative scores of wearable medical devices with respect to financial criteria are shown in Figure 35 below: 


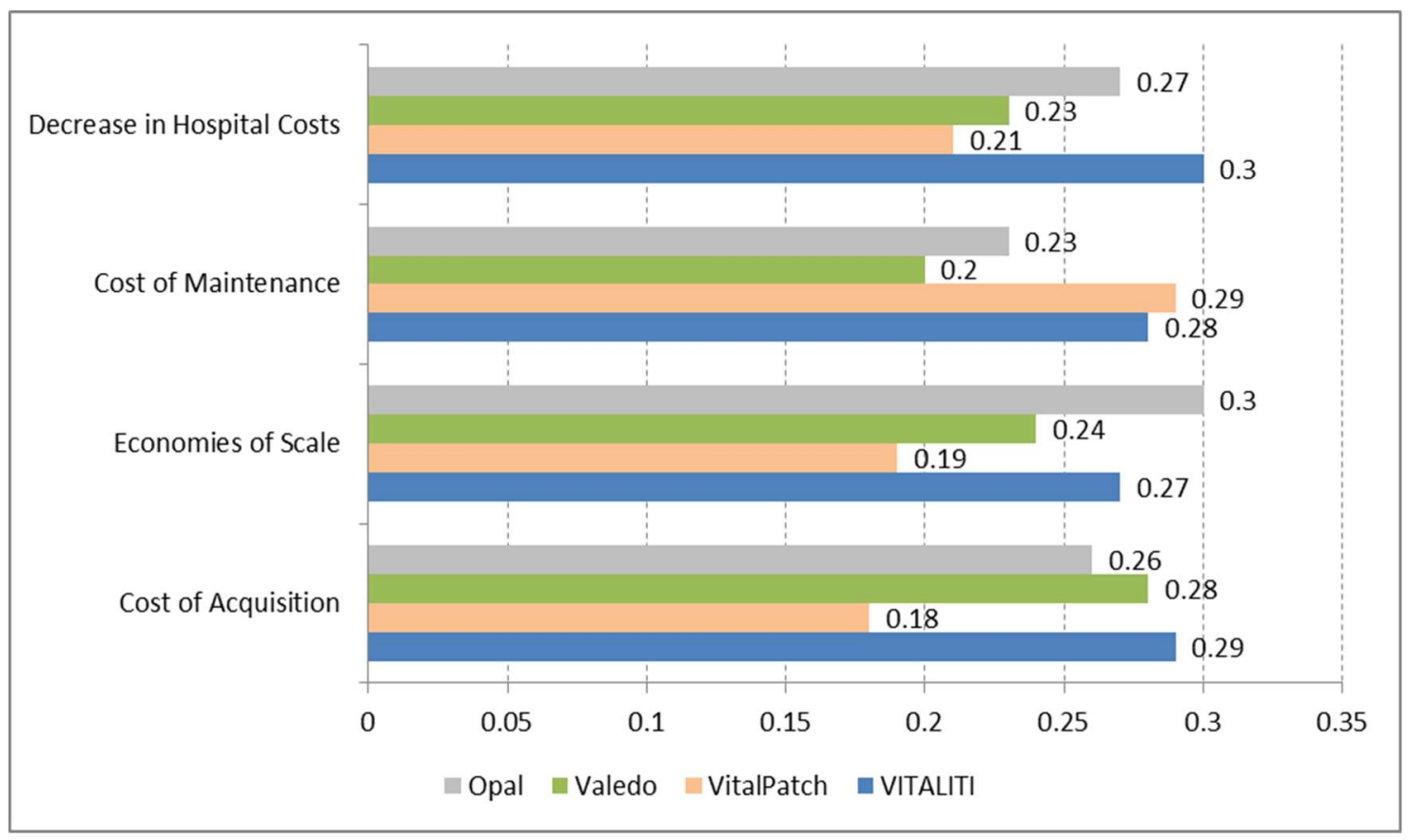

Figure 35 Relative importance of model alternatives with respect to criteria in Financial perspective

According to the results of expert panel 7.1, VITALITI has the highest value (29\%) with respect to cost of acquisition. The second place holds Valedo (28\%), followed by Opal (26\%) and VitalPatch(18\%). In comparisons of wearable medical devices with respect to economies of scale, Opal is a leading alternative (30\%), closely followed by VITALITI (27\%), Valedo (24\%) and VitalPatch (19\%). Alternatives rated with respect to 'cost of maintenance' criteria had the following lineup: VitalPatch (29\%), VITALITI (28\%), Opal (23\%) and Valedo (20\%). The following ranking of the alternatives was given by the experts with respect to decrease in hospital costs criterion: VITALITI (30\%), Opal (27\%), Valedo (23\%) and VitalPatch (21\%). 


\subsubsection{Analysis of expert panel 7.1 results}

All experts of panel 7.1 had inconsistencies within acceptable level. Some disagreement among the experts $(0.109)$ was present while comparing alternatives with respect to decrease in hospital costs and 2 experts disagreed while evaluating importance of VitalPatch and Valedo. One of the experts ranked Valedo and Opal the highest (0.32), followed by VITALITI (0.28) and giving the lowest rank to VitalPatch (0.09). The other expert gave VITALITI and VitalPatch the highest rank (0.32), while showing lower scores for Opal (0.22) and Valedo (0.14). Table 54 with individual relative priority weights, aggregated group results, inconsistency levels and group disagreement is shown below. 
Table 54 Analysis of results of expert panel 7.1

\begin{tabular}{|l|r|r|r|r|r|}
\hline Panel 7.1 & VITALITI & VitalPatch & Valedo & \multicolumn{1}{l|}{ Opal } & Inconsistency \\
\hline Expert 1.1 & 0.25 & 0.25 & 0.25 & 0.25 & 0.000 \\
\hline Expert 2.1 & 0.32 & 0.1 & 0.31 & 0.27 & 0.001 \\
\hline Mean & $\mathbf{0 . 2 9}$ & $\mathbf{0 . 1 8}$ & $\mathbf{0 . 2 8}$ & $\mathbf{0 . 2 6}$ & \\
\hline Disagreement & & & & & 0.060 \\
\hline \hline Expert 1.2 & 0.3 & 0.13 & 0.34 & 0.23 & 0.036 \\
\hline Expert 2.2 & 0.25 & 0.25 & 0.14 & 0.36 & 0.025 \\
\hline Mean & $\mathbf{0 . 2 7}$ & $\mathbf{0 . 1 9}$ & $\mathbf{0 . 2 4}$ & $\mathbf{0 . 3}$ & \\
\hline Disagreement & & & & & 0.095 \\
\hline \hline Expert 1.3 & 0.25 & 0.25 & 0.25 & 0.25 & 0.036 \\
\hline Expert 2.3 & 0.32 & 0.32 & 0.14 & 0.22 & 0.001 \\
\hline Mean & $\mathbf{0 . 2 8}$ & $\mathbf{0 . 2 9}$ & $\mathbf{0 . 2}$ & $\mathbf{0 . 2 3}$ & \\
\hline Disagreement & & & & & 0.053 \\
\hline \hline Expert 1.4 & 0.28 & 0.09 & 0.32 & 0.32 & 0.007 \\
\hline Expert 2.4 & 0.32 & 0.32 & 0.14 & 0.22 & 0.001 \\
\hline Mean & $\mathbf{0 . 3}$ & $\mathbf{0 . 2 1}$ & $\mathbf{0 . 2 3}$ & $\mathbf{0 . 2 7}$ & \\
\hline Disagreement & & & & & 0.109 \\
\hline
\end{tabular}

\subsubsection{Expert panel 7.2 results}

Experts of expert panel 7.2 analyzed technical criteria: 1) reliability; 2)

physician's ease of use; 3) productivity impact (usefulness); 4) interoperability; 5) ease of data access.

The inconsistencies of the expert opinions were within acceptable level. The arithmetic means of the relative scores of wearable medical devices with respect to technical criteria are shown in Figure 36 below: 


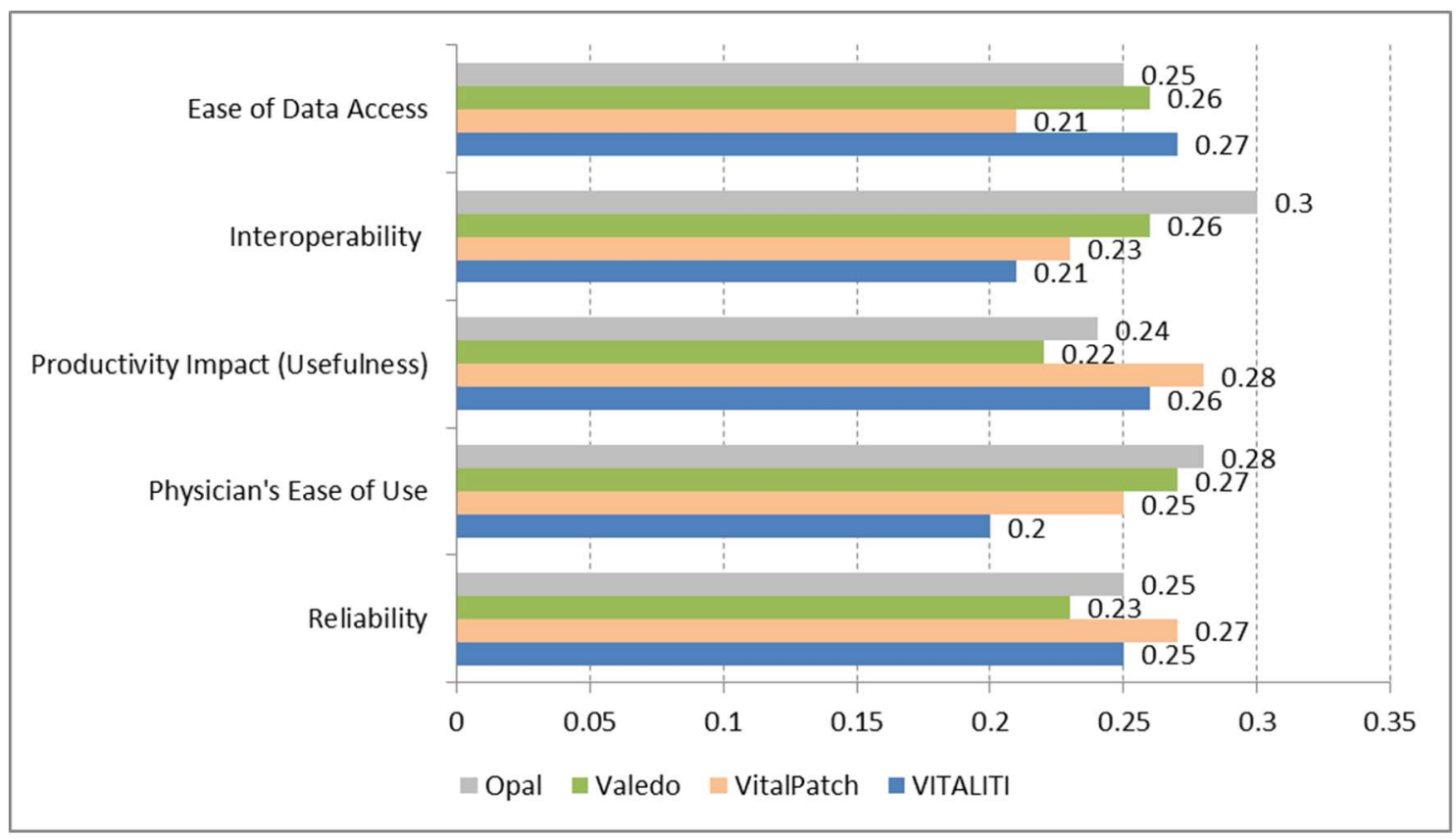

Figure 36 Relative importance of model alternatives with respect to criteria in Technical perspective

According to the results of expert panel 7.2, VitalPatch has the highest value $(27 \%)$ with respect to reliability. The second place share VITALITI (25\%) and Opal (25\%). Valedo has the last place with $23 \%$. With respect to physician's ease of use, Opal has the highest score of $28 \%$, then Valedo (27\%), VitalPatch (25\%) and VITALITI (20\%). In comparisons of wearable medical devices with respect to productivity impact (usefulness), VitalPatch is a leading alternative (28\%), closely followed by VITALITI (26\%), Opal (24\%) and Valedo (22\%).

Alternatives rated with respect to interoperability criteria had the following lineup: Opal (30\%), Valedo (26\%), VitalPatch (23\%) and VITALITI (21\%). The following ranking of the alternatives was given by the experts with respect to 'ease of data 
access' criterion: VITALITI (27\%), Valedo (26\%), Opal (25\%) and VitalPatch (21\%).

\subsubsection{Analysis of expert panel 7.2 results}

All experts of panel 7.2 had inconsistencies within acceptable level. The disagreement was also at acceptable level. Table 55 with individual relative priority weights, aggregated group results, inconsistency levels and group disagreement is shown below. 
Table 55 Analysis of results of expert panel 7.2

\begin{tabular}{|l|r|r|r|r|r|}
\hline Panel 7.2 & VITALITI & VitalPatch & \multicolumn{1}{l|}{ Valedo } & \multicolumn{1}{l|}{ Opal } & Inconsistency \\
\hline Expert 1.1 & 0.25 & 0.25 & 0.25 & 0.25 & 0.000 \\
\hline Expert 2.1 & 0.35 & 0.32 & 0.17 & 0.16 & 0.002 \\
\hline Expert 3.1 & 0.14 & 0.24 & 0.28 & 0.35 & 0.001 \\
\hline Mean & $\mathbf{0 . 2 5}$ & $\mathbf{0 . 2 7}$ & $\mathbf{0 . 2 3}$ & $\mathbf{0 . 2 5}$ & \\
\hline Disagreement & & & & & 0.081 \\
\hline \hline Expert 1.2 & 0.25 & 0.25 & 0.25 & 0.25 & 0.000 \\
\hline Expert 2.2 & 0.18 & 0.19 & 0.33 & 0.3 & 0.001 \\
\hline Expert 3.2 & 0.17 & 0.3 & 0.23 & 0.3 & 0.001 \\
\hline Mean & $\mathbf{0 . 2}$ & $\mathbf{0 . 2 5}$ & $\mathbf{0 . 2 7}$ & $\mathbf{0 . 2 8}$ & \\
\hline Disagreement & & & & & 0.045 \\
\hline \hline Expert 1.3 & 0.27 & 0.3 & 0.2 & 0.22 & 0.012 \\
\hline Expert 2.3 & 0.31 & 0.31 & 0.18 & 0.2 & 0.003 \\
\hline Expert 3.3 & 0.19 & 0.24 & 0.27 & 0.3 & 0.000 \\
\hline Mean & $\mathbf{0 . 2 6}$ & $\mathbf{0 . 2 8}$ & $\mathbf{0 . 2 2}$ & $\mathbf{0 . 2 4}$ & \\
\hline Disagreement & & & & & 0.049 \\
\hline \hline Expert 1.4 & 0.25 & 0.25 & 0.25 & 0.25 & 0.000 \\
\hline Expert 2.4 & 0.19 & 0.19 & 0.26 & 0.36 & 0.002 \\
\hline Expert 3.4 & 0.2 & 0.25 & 0.26 & 0.29 & 0.018 \\
\hline Mean & $\mathbf{0 . 2 1}$ & $\mathbf{0 . 2 3}$ & $\mathbf{0 . 2 6}$ & $\mathbf{0 . 3}$ & \\
\hline Disagreement & & & & & 0.038 \\
\hline \hline Expert 1.5 & 0.25 & 0.25 & 0.25 & 0.25 & 0.000 \\
\hline Expert 2.5 & 0.34 & 0.16 & 0.3 & 0.2 & 0.003 \\
\hline Expert 3.5 & 0.22 & 0.23 & 0.24 & 0.31 & 0.019 \\
\hline Mean & $\mathbf{0 . 2 7}$ & $\mathbf{0 . 2 1}$ & $\mathbf{0 . 2 6}$ & $\mathbf{0 . 2 5}$ & \\
\hline Disagreement & & & & & 0.05 \\
\hline
\end{tabular}

\subsubsection{Expert panel 7.3 results}

Experts of expert panel 7.3 analyzed organizational criteria: 1) training needed; 2) tech support; 3) data support; 4) competitive advantage.

The inconsistencies of the expert opinions were within acceptable level. The arithmetic means of the relative scores of wearable medical devices with respect to organizational criteria are shown in Figure 37 below: 


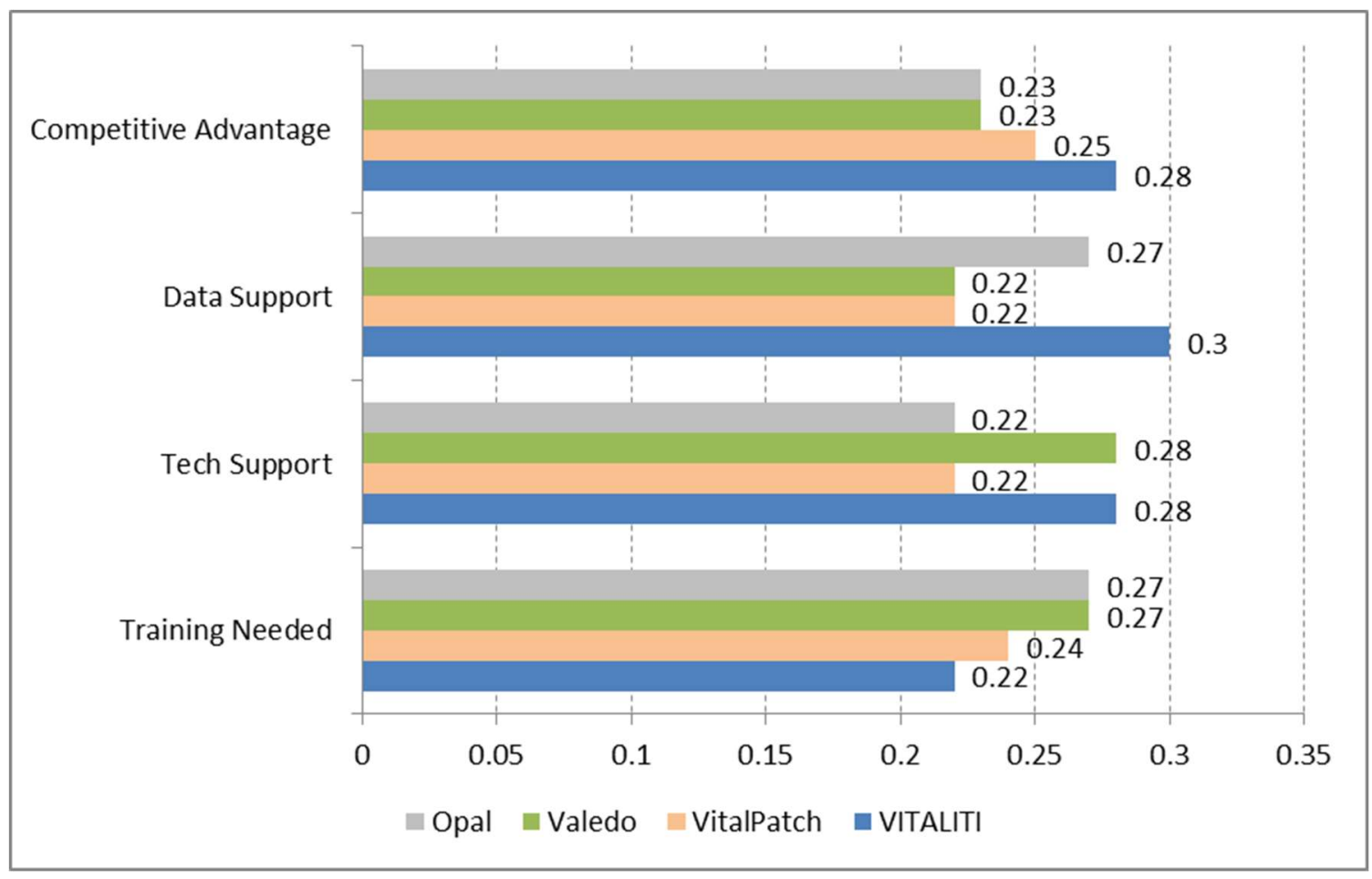

Figure 37 Relative importance of model alternatives with respect to criteria in Organizational perspective

According to the results of expert panel 7.3 , Opal $(27 \%)$ and Valedo $(27 \%)$ share the first place with respect to criterion 'training needed'. VitalPatch (24\%) and VITALITI (22\%) are the bottom two. The following ranking of the alternatives was given by the experts with respect to 'tech support' criterion: VITALITI (28\%), Valedo (28\%), Opal (22\%) and VitalPatch (22\%). In comparisons of wearable medical devices with respect to 'data support', VITALITI is a leading alternative (30\%), closely followed by Opal (27\%), Valedo (22\%) and VitalPatch (22\%). With respect to 'competitive advantage', VITALITI has the highest score of $28 \%$, then VitalPatch (25\%), Valedo (23\%), and Opal (23\%). 


\subsubsection{Analysis of expert panel 7.3 results}

All experts of panel 7.3 had inconsistencies and disagreements within acceptable level. The table with individual relative priority weights, aggregated group results, inconsistency levels and group disagreement is shown below (Table 56).

Table 56 Analysis of results of expert panel 7.3

\begin{tabular}{|l|r|r|r|r|r|}
\hline Panel 7.3 & VITALITI & VitalPatch & Valedo & \multicolumn{1}{l|}{ Opal } & Inconsistency \\
\hline Expert 1.1 & 0.25 & 0.25 & 0.25 & 0.25 & 0.000 \\
\hline Expert 2.1 & 0.19 & 0.23 & 0.28 & 0.29 & 0.000 \\
\hline Mean & $\mathbf{0 . 2 2}$ & $\mathbf{0 . 2 4}$ & $\mathbf{0 . 2 7}$ & $\mathbf{0 . 2 7}$ & \\
\hline Disagreement & & & & & 0.028 \\
\hline \hline Expert 1.2 & 0.25 & 0.25 & 0.25 & 0.25 & 0.000 \\
\hline Expert 2.2 & 0.3 & 0.2 & 0.3 & 0.2 & 0.000 \\
\hline Mean & $\mathbf{0 . 2 8}$ & $\mathbf{0 . 2 2}$ & $\mathbf{0 . 2 8}$ & $\mathbf{0 . 2 2}$ & \\
\hline Disagreement & & & & & 0.035 \\
\hline \hline Expert 1.3 & 0.25 & 0.25 & 0.25 & 0.25 & 0.000 \\
\hline Expert 2.3 & 0.35 & 0.19 & 0.19 & 0.28 & 0.000 \\
\hline Mean & $\mathbf{0 . 3}$ & $\mathbf{0 . 2 2}$ & $\mathbf{0 . 2 2}$ & $\mathbf{0 . 2 7}$ & \\
\hline Disagreement & & & & & 0.048 \\
\hline \hline Expert 1.4 & 0.25 & 0.25 & 0.25 & 0.25 & 0.000 \\
\hline Expert 2.4 & 0.32 & 0.26 & 0.21 & 0.21 & 0.000 \\
\hline Mean & $\mathbf{0 . 2 8}$ & $\mathbf{0 . 2 5}$ & $\mathbf{0 . 2 3}$ & $\mathbf{0 . 2 3}$ & \\
\hline Disagreement & & & & & 0.031 \\
\hline
\end{tabular}

\subsubsection{Expert panel 7.4 results}

Experts of expert panel 7.4 analyzed patient criteria: 1) patient experience; 2) clinical benefits for patients; 3) privacy/security. 
The inconsistencies of the expert opinions were within acceptable level. The arithmetic means of the relative scores of wearable medical devices with respect to patient criteria are shown in Figure 38 below:

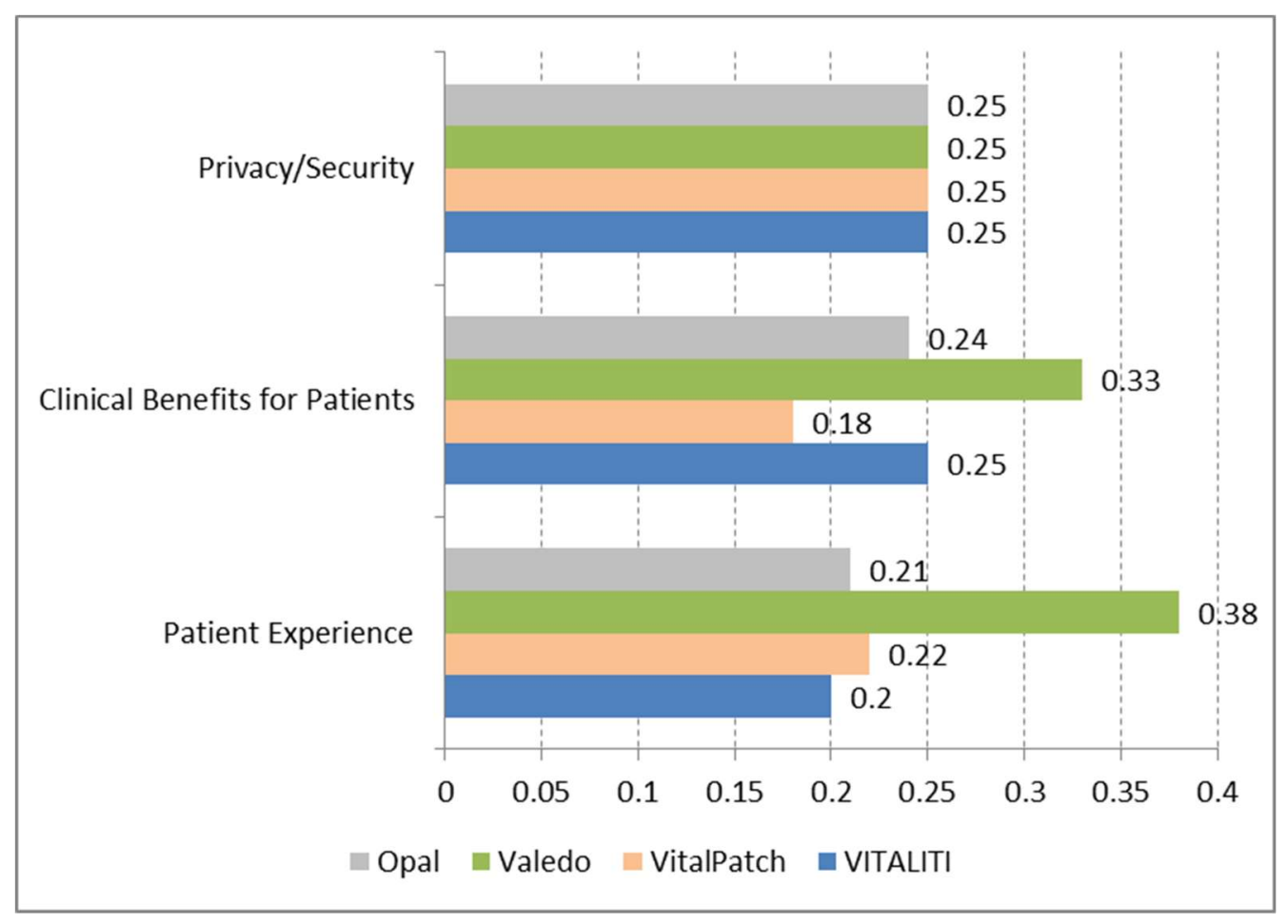

Figure 38 Relative importance of model alternatives with respect to criteria in Patient perspective

According to the results of expert panel 7.4 , Valedo has the highest ranking of $38 \%$ with respect to criterion 'patient experience'. The device gives patients an opportunity to play videogames for therapy. VitalPatch (22\%), Opal (21\%) and VITALITI (20\%) have distant second, third and fourth ranking with respect to 'patient experience'. Valedo also ranked first in criterion 'clinical benefits to patients', since it aimed to provide back pain therapy in addition to 
movement data collection and analysis. VITALITI (25\%) has the second place in 'clinical benefits for patients' category, closely followed by Opal (24\%) and VitalPatch (18\%). In comparisons of wearable medical devices with respect to 'privacy/security', all the devices received equal score of $25 \%$ each. All of the device manufacturers stress the importance of privacy and security of their products and the experts also ranked them equal in that category.

\subsubsection{Analysis of expert panel 7.4 results}

All experts of panel 7.4 had inconsistencies and disagreements within acceptable level. Table 57 with individual relative priority weights, aggregated group results, inconsistency levels and group disagreement is shown below.

Table 57 Analysis of results of expert panel 7.4

\begin{tabular}{|l|r|r|r|r|r|}
\hline Panel 7.4 & VITALITI & VitalPatch & Valedo & \multicolumn{1}{l|}{ Opal } & Inconsistency \\
\hline Expert 1.1 & 0.19 & 0.18 & 0.5 & 0.16 & 0.033 \\
\hline Expert 2.1 & 0.25 & 0.25 & 0.25 & 0.25 & 0.000 \\
\hline Mean & $\mathbf{0 . 2}$ & $\mathbf{0 . 2 2}$ & $\mathbf{0 . 3 8}$ & $\mathbf{0 . 2 1}$ & \\
\hline Disagreement & & & & & 0.103 \\
\hline \hline Expert 1.2 & 0.26 & 0.1 & 0.42 & 0.22 & 0.036 \\
\hline Expert 2.2 & 0.25 & 0.25 & 0.25 & 0.25 & 0.000 \\
\hline Mean & $\mathbf{0 . 2 5}$ & $\mathbf{0 . 1 8}$ & $\mathbf{0 . 3 3}$ & $\mathbf{0 . 2 4}$ & \\
\hline Disagreement & & & & & 0.079 \\
\hline \hline Expert 1.3 & 0.25 & 0.25 & 0.25 & 0.25 & 0.000 \\
\hline Expert 2.3 & 0.25 & 0.25 & 0.25 & 0.25 & 0.000 \\
\hline Mean & $\mathbf{0 . 2 5}$ & $\mathbf{0 . 2 5}$ & $\mathbf{0 . 2 5}$ & $\mathbf{0 . 2 5}$ & \\
\hline Disagreement & & & & & 0.000 \\
\hline \hline
\end{tabular}




\subsubsection{Expert panel 7.5 results}

Experts of expert panel 7.5 analyzed criteria of interpersonal perspective:

1) efficiency of communication and patient access; 2) information sharing; 3)

educational benefits and new knowledge creation.

The inconsistencies of the expert opinions were within acceptable level.

The arithmetic means of the relative scores of wearable medical devices with respect to interpersonal criteria are shown in Figure 39 below:

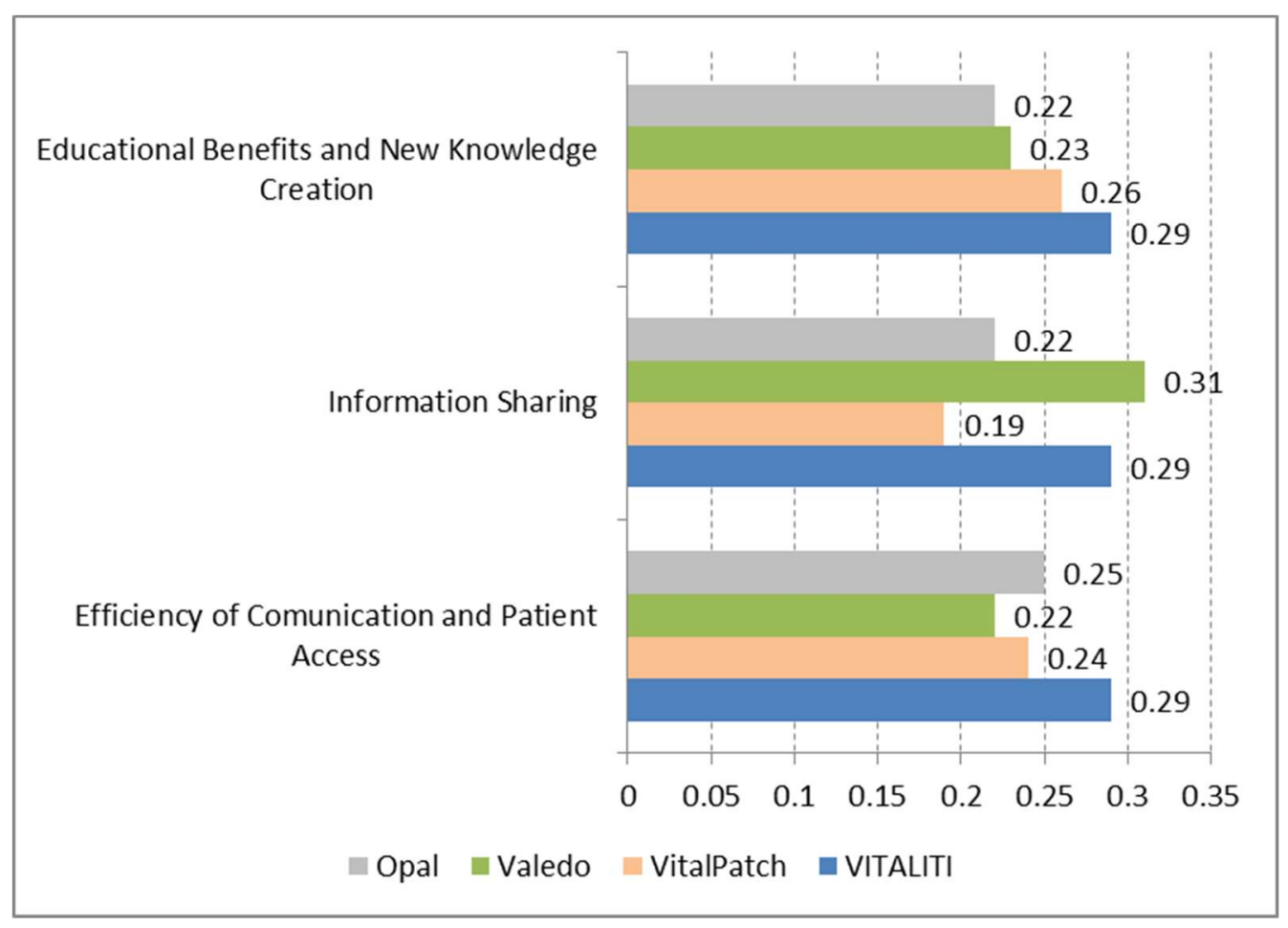

Figure 39 Relative importance of model alternatives with respect to criteria in Interpersonal perspective

According to the results of expert panel 7.5, VITALITI (29\%) has the highest ranking with respect to 'efficiency of communication and patient access' 
criterion, closely followed by Opal (25\%), VitalPatch (24\%) and Valedo (22\%). The following ranking of the alternatives was given by the experts with respect to 'information sharing' criterion: Valedo (31\%), VITALITI (29\%), Opal (22\%) and VitalPatch (19\%). In comparisons of wearable medical devices with respect to 'educational benefits and new knowledge creation', VITALITI is a leading alternative (29\%), followed by VitalPatch (26\%), Valedo (23\%) and Opal (22\%).

\subsubsection{Analysis of expert panel 7.5 results}

All experts of panel 7.5 had inconsistencies within acceptable level. There was some disagreement in experts, who compared wearable medical devices with respect to 'information sharing' criterion (0.110). Expert 1.2 (table below) gave very high ranking to Valedo (0.51) and placed VITALITI second (0.24), Opal - third (0.15) and VitalPatch - fourth (0.1). This expert is in subgroup A of panel 7.5-2. Subgroup B of panel 7.5-2 consists of experts 2.2 and 2.3. Their group disagreement value is within norm (0.058) and reflected in the table below. The experts in this subgroup gave the highest ranking to VITALITI (31\%), followed by Opal (25\%), VitalPatch (23\%) and Valedo (20\%). Table 58 with individual relative priority weights, aggregated group results, inconsistency levels and group disagreement is shown below. 
Table 58 Analysis of results of expert panel 7.5

\begin{tabular}{|l|r|r|r|r|r|}
\hline Panel 7.5 & VITALITI & VitalPatch & Valedo & \multicolumn{1}{l|}{ Opal } & Inconsistency \\
\hline Expert 1.1 & 0.25 & 0.25 & 0.25 & 0.25 & 0.000 \\
\hline Expert 2.1 & 0.25 & 0.25 & 0.25 & 0.25 & 0.000 \\
\hline Expert 3.1 & 0.38 & 0.21 & 0.16 & 0.25 & 0.001 \\
\hline Mean & $\mathbf{0 . 2 9}$ & $\mathbf{0 . 2 4}$ & $\mathbf{0 . 2 2}$ & $\mathbf{0 . 2 5}$ & \\
\hline Disagreement & & & & & 0.047 \\
\hline \hline Subgroup A & & & & & \\
\hline Expert 1.2 & 0.24 & 0.1 & 0.51 & 0.15 & 0.033 \\
\hline Subgroup B & & & & & \\
\hline Expert 2.2 & 0.25 & 0.25 & 0.25 & 0.25 & 0.000 \\
\hline Expert 3.2 & 0.38 & 0.21 & 0.16 & 0.25 & 0.001 \\
\hline $\begin{array}{l}\text { Subgroup B } \\
\text { Mean }\end{array}$ & $\mathbf{0 . 3 1}$ & $\mathbf{0 . 2 3}$ & $\mathbf{0 . 2}$ & $\mathbf{0 . 2 5}$ & \\
\hline $\begin{array}{l}\text { Subgroup B } \\
\text { Disagreement }\end{array}$ & & & & & 0.058 \\
\hline Mean & $\mathbf{0 . 2 9}$ & $\mathbf{0 . 1 9}$ & $\mathbf{0 . 3 1}$ & $\mathbf{0 . 2 2}$ & \\
\hline Disagreement & & & & & 0.110 \\
\hline \hline Expert 1.3 & 0.22 & 0.28 & 0.27 & 0.22 & 0.016 \\
\hline Expert 2.3 & 0.25 & 0.25 & 0.25 & 0.25 & 0.000 \\
\hline Expert 3.3 & 0.4 & 0.26 & 0.16 & 0.18 & 0.001 \\
\hline Mean & $\mathbf{0 . 2 9}$ & $\mathbf{0 . 2 6}$ & $\mathbf{0 . 2 3}$ & $\mathbf{0 . 2 2}$ & \\
\hline Disagreement & & & & & 0.058 \\
\hline \hline
\end{tabular}

\subsection{Synthesis of Priorities}

According to the results of expert panels, synthesis of priorities is calculated for different levels of the decision hierarchy. The relative priorities of technology assessment perspectives with respect to the mission are calculated according to panel 1 results. The relative priorities of technology assessment criteria with respect to the mission, relative importance of program alternatives with respect to criteria and perspectives and overall importance of wearable medical devices with respect to the mission of assessment of technology 
adoption potential of those devices in neurosurgery and orthopedics is presented in this section.

\subsubsection{Relative importance of criteria with respect to the mission}

The highest weighted criteria are Clinical Benefits for Patients (0.098), Patient Experience (0.078) and Privacy and Security (0.072). It is important to remark that the top three criteria belong to Patient perspective. Reliability $(0.058)$ is the next highest weighted criteria and it is a part of Technical perspective. There are some reliability issues with new technology and wearable devices in healthcare industry and it resonated with the experts in this study. Efficiency of Communication and Patient Access (0.054), which is a part of Interpersonal perspective, is very closely followed by financial criteria: Cost of Acquisition (0.054) and Cost of Maintenance (0.054) and interpersonal criterion - Information Sharing (0.053). The lowest weight has organizational criterion -- Competitive Advantage (0.030). While clinics realize the benefits of having competitive advantage in the marketplace when adopting new technology, compared to the other factors in the model, experts gave this criterion the lowest importance relative to the mission. Other criteria with lower rankings are Interoperability (0.036), Productivity Impact (Usefulness) (0.041), Physician's Ease of Use (0.042) and Educational Benefits and New Knowledge Creation (0.042). Relative importance of criteria with respect to the mission is presented in Figure 40 below: 


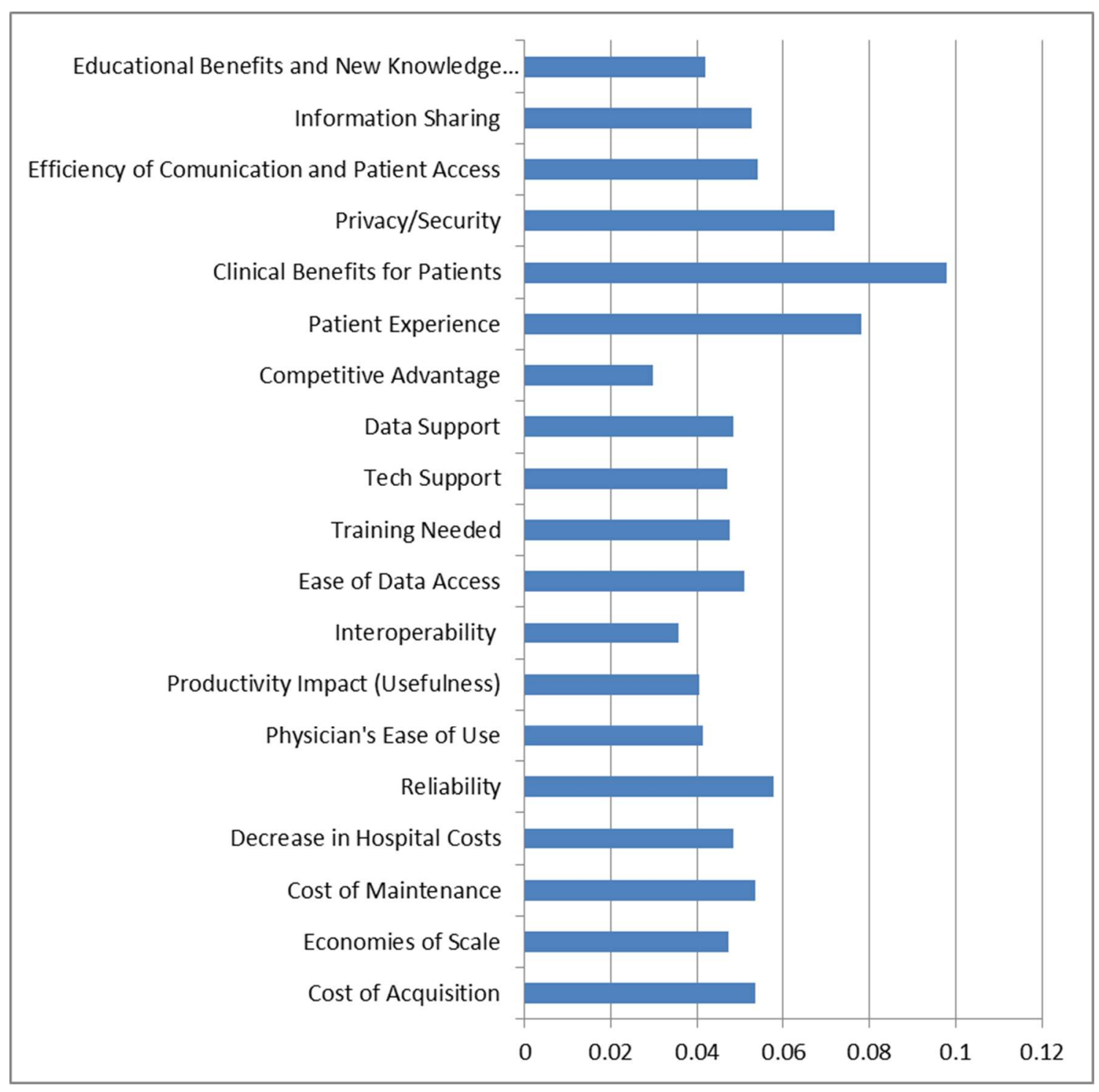

Figure 40 Relative importance of criteria with respect to the mission

\subsubsection{Relative importance of alternatives with respect to perspectives.}

This section shows relative importance of alternatives with respect to perspectives. This analysis provides the alignment of alternatives from our experts' point of view in each perspective. This analysis could be useful if clinicians would want to look at adoption of wearable from the single perspective 
view. The results are shown in five figures below (Figure 41, Figure 42, Figure 43, Figure 44 and Figure 45).

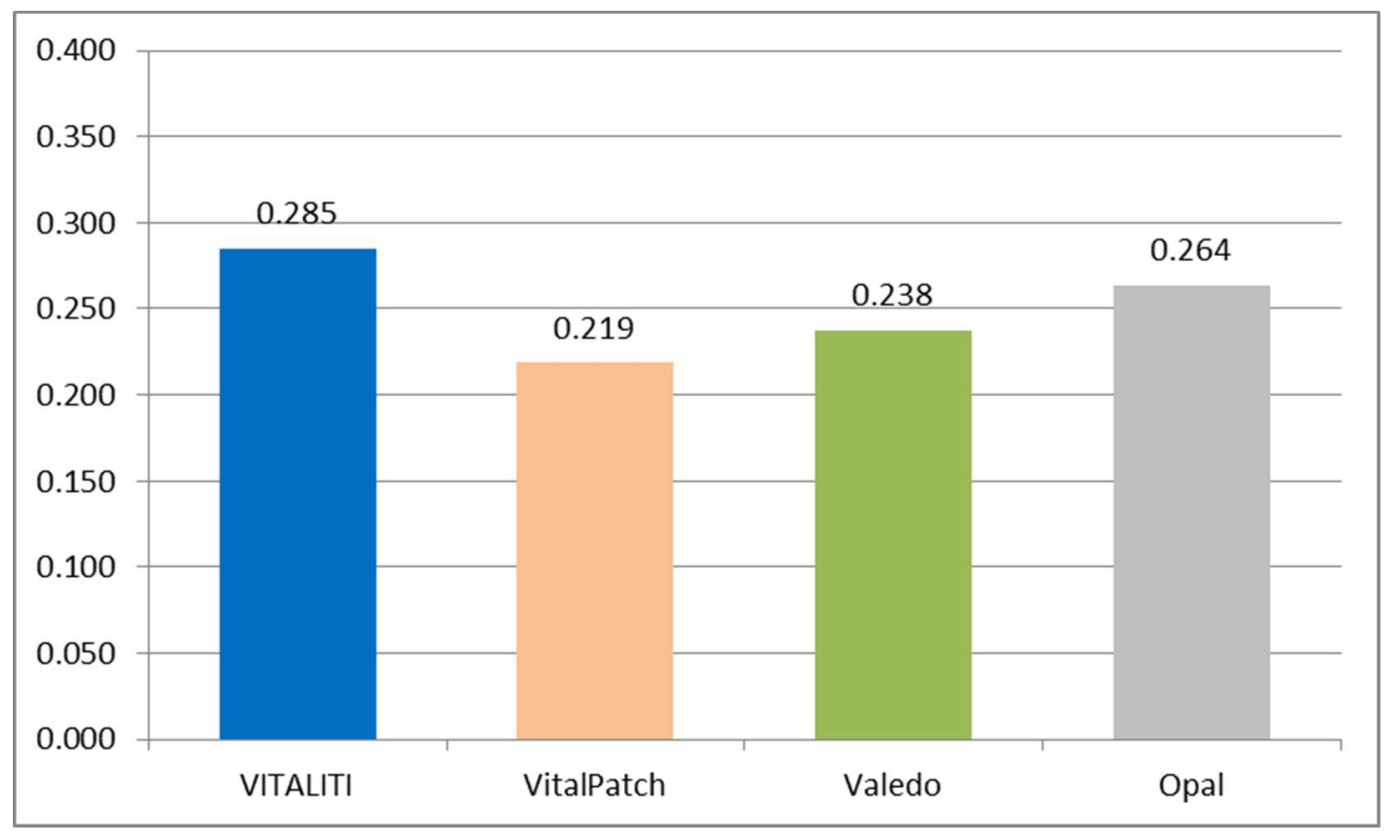

Figure 41 Relative importance of model alternatives with respect to Financial perspective

According to the results (Figure 41) of expert panels and considering Financial perspective, the relative importance leader is VITALITI $(0.285)$ and Opal is a close second (0.264). From our experts' point of view, those alternatives best reflect financial considerations according to the mission. Valedo (0.238) and VitalPatch (0.219) have lower scores. 


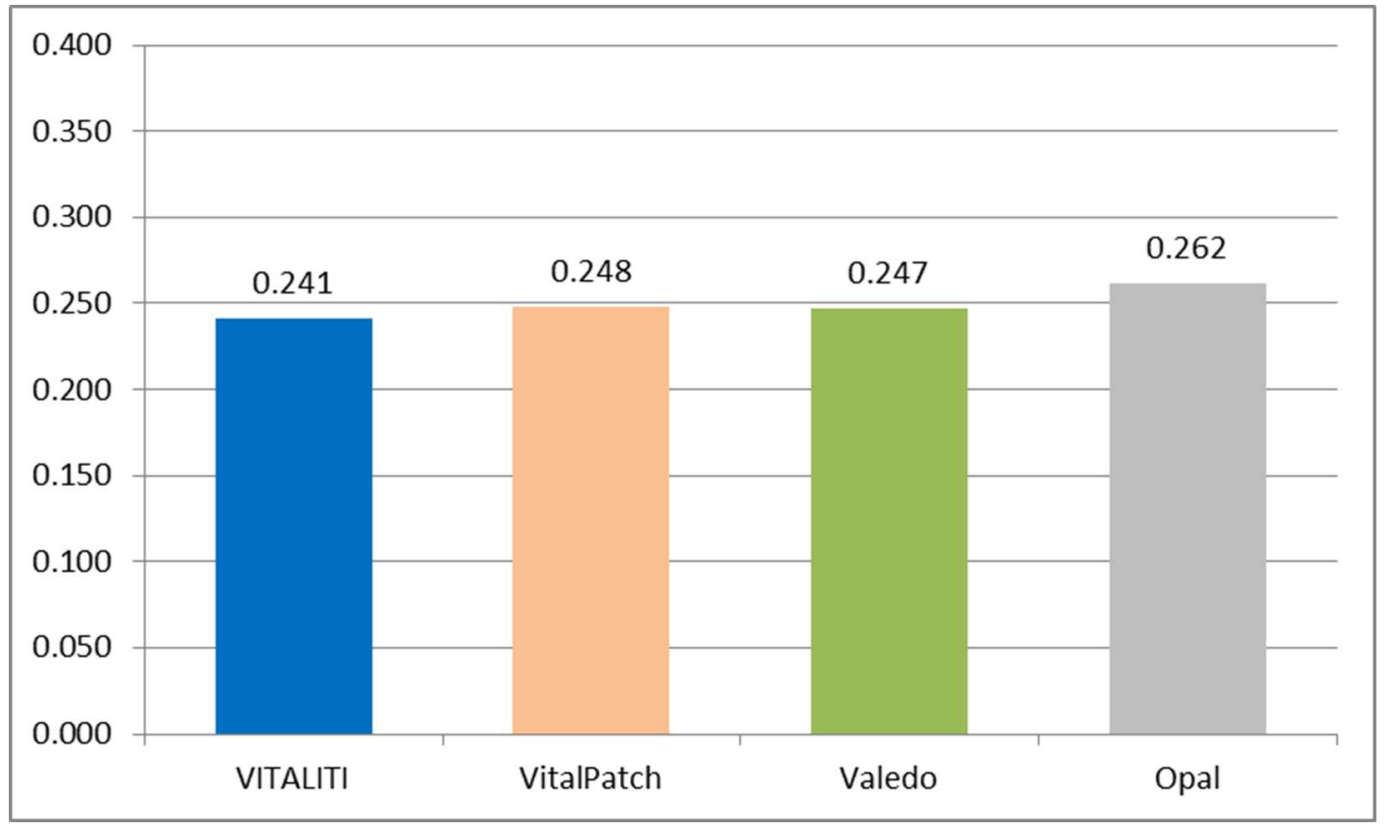

Figure 42 Relative importance of model alternatives with respect to Technical perspective

Our wearable medical devices show very strong technical values. While each of them shows higher or lower relative importance values in the five technical criteria, the combined picture (Figure 42) shows that Opal $(0.262)$ is a top alternative, while VitalPatch (0.248), Valedo (0.247) and VITALITI (0.241) have very similar results. 


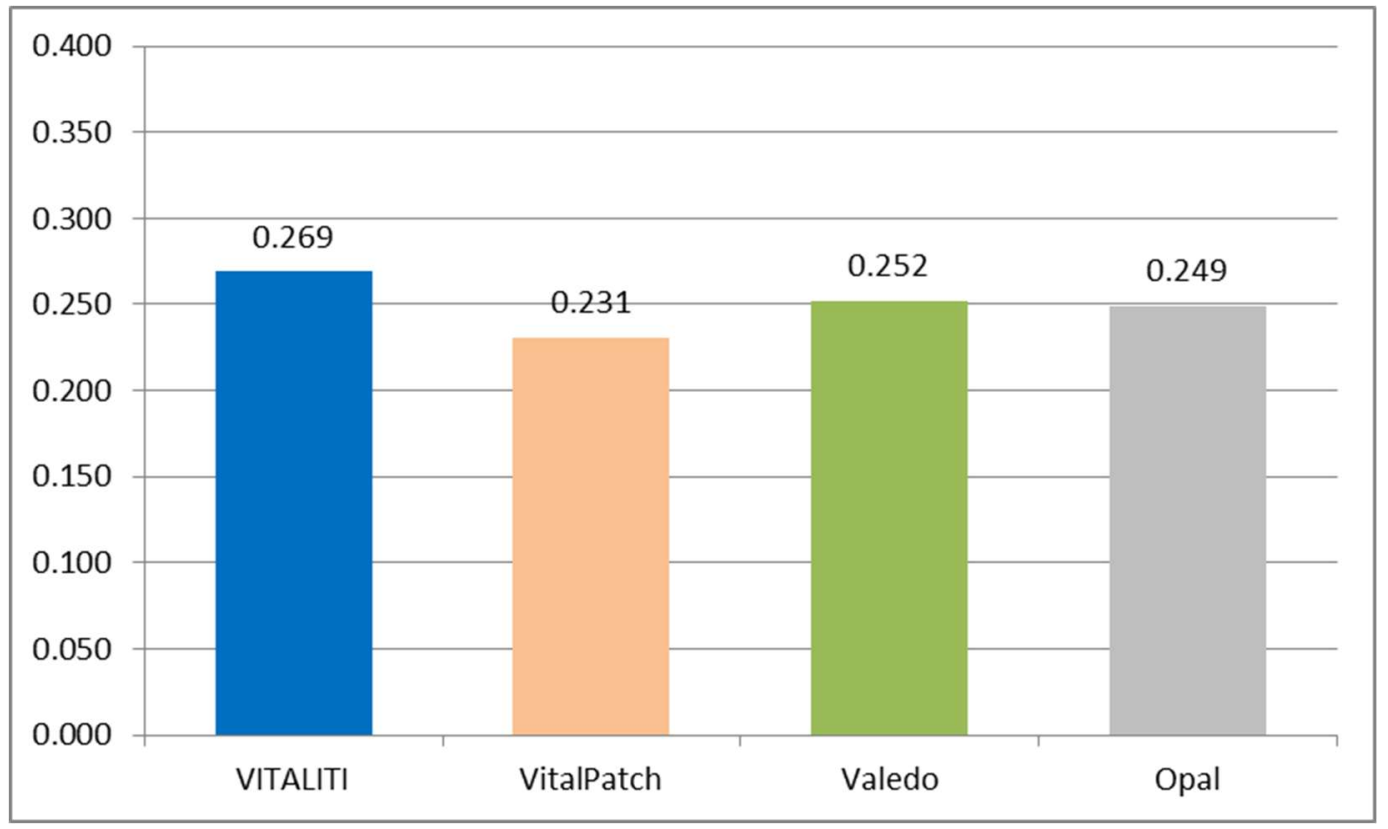

Figure 43 Relative importance of model alternatives with respect to Organizational perspective

According to the results (Figure 43) of expert panel 4 and expert panel 7.3, VITALITI has the highest relative importance $(0.269)$ with respect to Organizational perspective of technology adoption potential. Valedo (0.252) and Opal (0.249) closely follow. VitalPatch has the lowest relative importance score of 0.231 . 


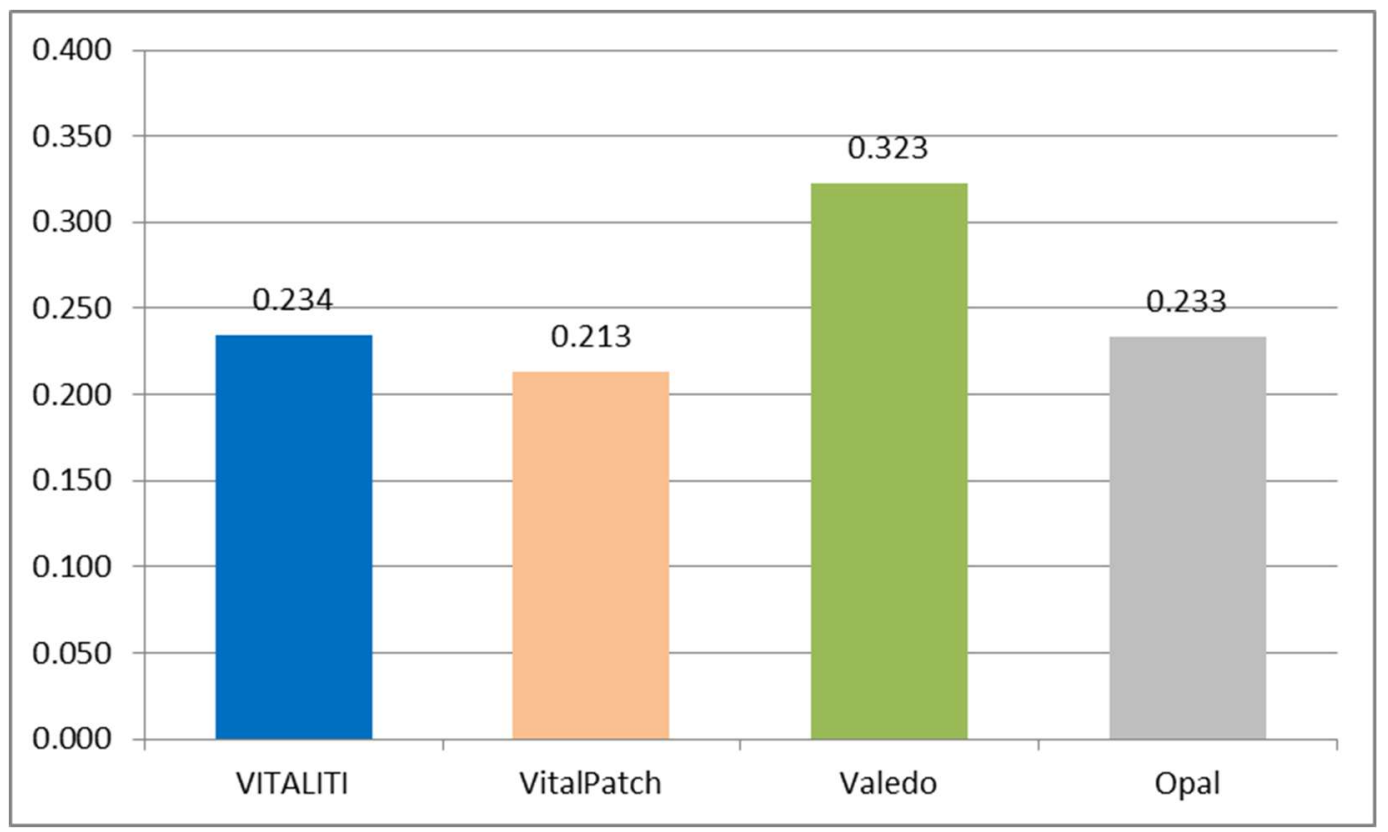

Figure 44 Relative importance of model alternatives with respect to Patient perspective

According to the results (Figure 44), Valedo (0.323) has the highest relative importance of model alternatives with respect to Patient perspective.

Valedo gives an option of performing some exercises through games, which may be helpful and engaging to patients with certain spine health conditions. VITALITI (0.234) and Opal (0.233) are close in ranks for the second place, while VitalPatch (0.213) has the lowest relative importance value with respect to Patient perspective. 


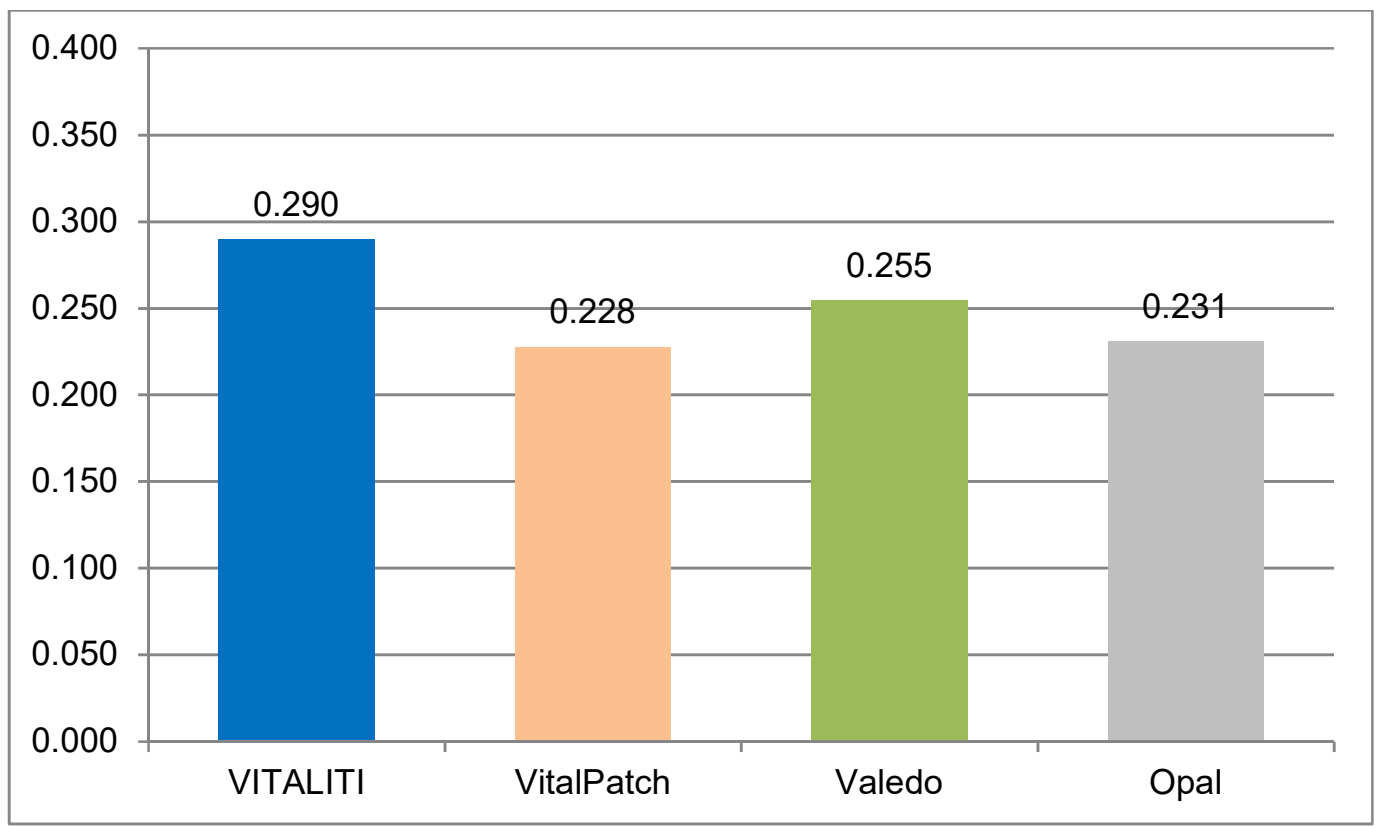

Figure 45 Relative importance of model alternatives with respect to Interpersonal perspective

The results (Figure 45) show that VITALITI (0.290) has the highest relative importance values with respect to Interpersonal perspective. Valedo is a close second with the value of 0.255 . Opal (0.231) and VitalPatch (0.228) have similar values and have third and fourth places accordingly. So, from our experts' interpersonal viewpoint, VITALITI has the highest adoption potential, while VitalPatch has the lowest.

\subsubsection{Overall importance of alternatives with respect to the mission.}

This section provides analysis of the global priorities of model alternatives with respect to the mission. The ranking of the wearable sensor products is shown as well as their technology adoption potential for pervasive care in 
neurosurgery and orthopedics. The overall importance of model alternatives with respect to the mission is shown in Figure 46.

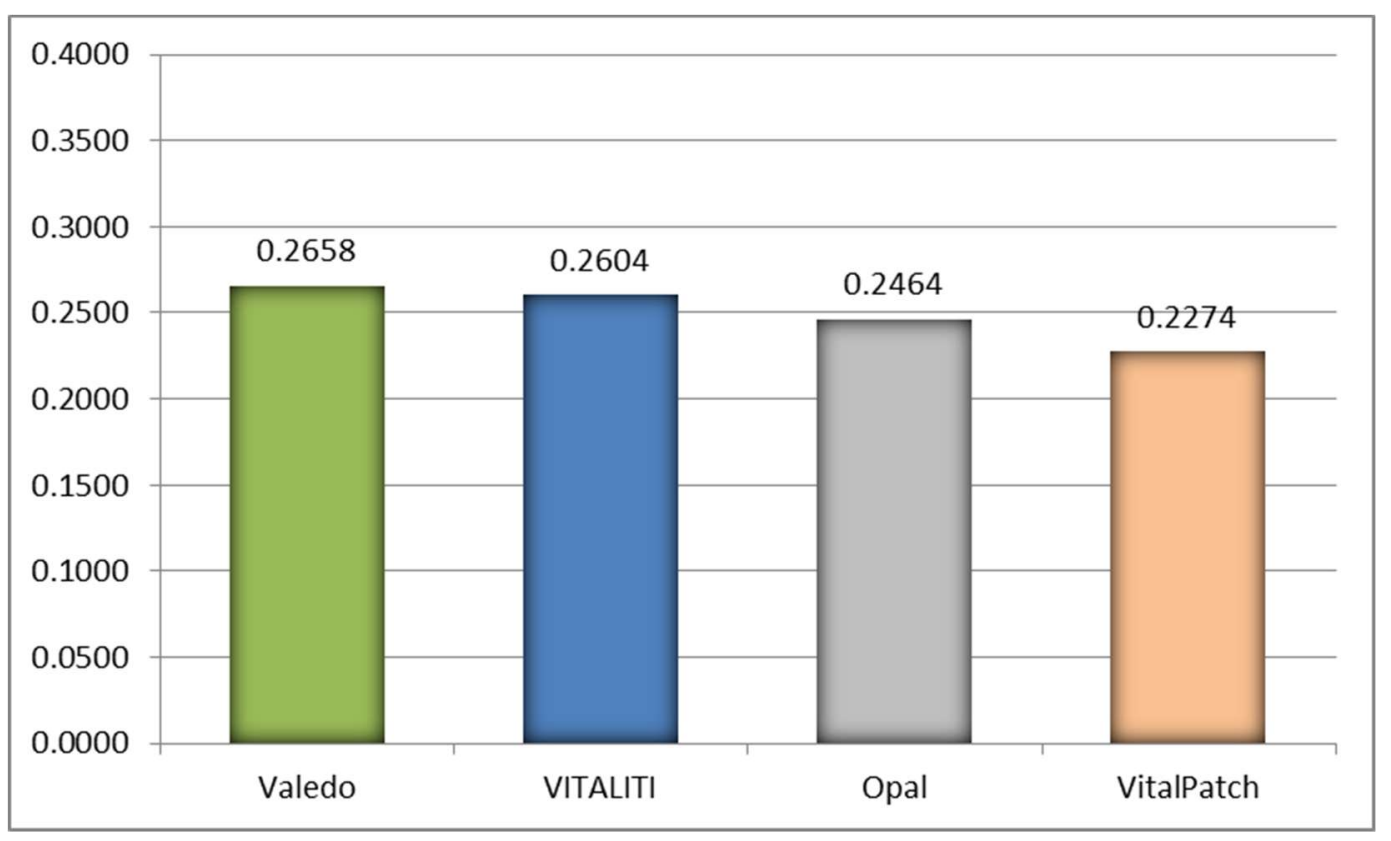

Figure 46 Overall importance of model alternatives with respect to the mission

According to the results from all the expert panels, Valedo $(0.2658)$ has the highest ranking and thus the highest technology adoption potential for pervasive care in neurosurgery and orthopedics. VITALITI (0.2604) has the second global importance ranking with respect to the mission, while Opal $(0.2464)$ and VitalPatch $(0.2274)$ have the third and the fourth overall importance accordingly. As it was shown in the previous chapter, Valedo and VITALITI had higher importance ranking in several perspectives. However, it's important to admit that all the analyzed wearable sensor products had high importance score and are valuable adoption potential contenders for pervasive care in neurosurgery and orthopedics. 


\subsection{Analysis of Ranking of Model Alternatives with Respect to Expert Panels Disagreements}

There were some disagreements in Panels 1-6. Most of the disagreements were within 0.1 , with Panel 2 having the highest disagreement among the experts -0.18 . This section analyses the impact of those disagreements on the overall ranking of model alternatives. The ranking of model alternatives are recalculated with each subgroup compared with the original importance values of model alternatives.

\subsubsection{Analysis of results with respect to expert disagreements in panel 1}

Experts in panel 1 disagree on the relative importance of perspectives with respect to overall mission. Hierarchical clustering analysis distinguished two subgroups in expert panel 1, which lowered the disagreement values. Table 59 below shows the relative importance of perspectives with respect to the mission.

Table 59 Relative importance of perspectives in panel 1 and its subgroups

\begin{tabular}{|l|r|r|r|r|r|}
\hline & Financial & Technical & Organizational & Patient & Interpersonal \\
\hline Panel 1 & 0.203 & 0.227 & 0.173 & 0.248 & 0.149 \\
\hline Subgroup A & 0.28 & 0.21 & 0.28 & 0.08 & 0.15 \\
\hline Subgroup B & 0.16 & 0.24 & 0.11 & 0.35 & 0.15 \\
\hline
\end{tabular}

The rankings of alternatives were recalculated according to results of 2 subgroups and compared to original rankings of expert panel 1. Synthesis of priorities and rankings of alternatives with subgroups compared to overall expert panel 1 results are presented in Table 60 below. 
Table 60 Global rankings of model alternatives of expert panel 1, subgroups A and $\mathrm{B}$

\begin{tabular}{|l|r|l|l|r|}
\hline & VITALITI & VitalPatch & Valedo & Opal \\
\hline Panel 1 & 2 & 4 & 1 & 3 \\
\hline Subgroup A & 1 & 4 & 2 & 3 \\
\hline Subgroup B & 2 & 4 & 1 & 3 \\
\hline
\end{tabular}

Only rankings of Valedo and VITALITI switch places in Subgroup A

results. Valedo and VITALITI are very close in ranking overall with 0.2658 and

0.2604 respectively. With Subgroup A the values become 0.268 for VITALITI and 0.253 for Valedo. Other rankings remain the same.

\subsubsection{Analysis of results with respect to expert disagreements in panel 2}

Experts in panel 2 disagree on the relative importance of financial criteria in Financial perspective. Hierarchical clustering analysis distinguished four subgroups in panel 2 , which lowered the disagreement values. Table 61 below shows the relative importance of criteria in Financial perspective.

Table 61 Relative importance of criteria in expert panel 2 and its subgroups

\begin{tabular}{|c|c|c|c|c|}
\hline & $\begin{array}{l}\text { Cost of } \\
\text { Acquisition }\end{array}$ & $\begin{array}{l}\text { Economies } \\
\text { of Scale }\end{array}$ & $\begin{array}{l}\text { Cost of } \\
\text { Maintenance }\end{array}$ & $\begin{array}{l}\text { Decrease in Hospital } \\
\text { Costs }\end{array}$ \\
\hline Panel 2 & 0.264 & 0.233 & 0.264 & 0.24 \\
\hline Subgroup A & 0.18 & 0.15 & 0.66 & 0.01 \\
\hline Subgroup B & 0.56 & 0.15 & 0.2 & 0.09 \\
\hline Subgroup C & 0.15 & 0.54 & 0.14 & 0.17 \\
\hline Subgroup D & 0.19 & 0.14 & 0.26 & 0.41 \\
\hline
\end{tabular}

The rankings of alternatives were recalculated according to results of 4 subgroups and compared to original rankings of panel 2. Synthesis of priorities 
and rankings of alternatives with subgroups compared to overall panel 2 results are presented in Table 62 below.

Table 62 Global rankings of model alternatives of panel 2 and subgroups A, B, C and D

\begin{tabular}{|l|r|l|r|r|}
\hline & VITALITI & VitalPatch & Valedo & Opal \\
\hline Panel 1 & 2 & 4 & 1 & 3 \\
\hline Subgroup A & 2 & 4 & 1 & 3 \\
\hline Subgroup B & 2 & 4 & 1 & 3 \\
\hline Subgroup C & 2 & 4 & 1 & 3 \\
\hline Subgroup D & 2 & 4 & 1 & 3 \\
\hline
\end{tabular}

Rankings of the model alternatives remain unchanged with every subgroup compared to the complete panel 2 results. Valedo still has the highest importance ranking, followed by VITALITI, Opal and VitalPatch.

\subsubsection{Analysis of results with respect to expert disagreements in panel 3}

Experts in expert panel 3 disagree on the relative importance of technical criteria in Technical perspective. Hierarchical clustering analysis distinguished two subgroups in panel 3 , which lowered the disagreement values. Table 63 shows the relative importance of criteria in Technical perspective.

Table 63 Relative importance of criteria in expert panel 3 and its subgroups

\begin{tabular}{|l|r|r|r|r|r|}
\hline & Reliability & $\begin{array}{l}\text { Physician's } \\
\text { Ease of } \\
\text { Use }\end{array}$ & $\begin{array}{l}\text { Productivity } \\
\text { Impact } \\
\text { (Usefulness) }\end{array}$ & Interoperability & \multicolumn{2}{l|}{$\begin{array}{l}\text { Ease of Data } \\
\text { Access }\end{array}$} \\
\hline Panel 3 & 0.255 & 0.183 & 0.179 & 0.158 & 0.225 \\
\hline Subgroup A & 0.15 & 0.21 & 0.12 & 0.22 & 0.3 \\
\hline Subgroup B & 0.36 & 0.15 & 0.24 & 0.1 & 0.15 \\
\hline
\end{tabular}


The rankings of alternatives were recalculated according to results of 2 subgroups and compared to total rankings in panel 3 . Synthesis of priorities and rankings of alternatives with subgroups compared to overall panel 3 results are presented in Table 64 below.

Table 64 Global rankings of model alternatives of panel 3 and its subgroups A and $\mathrm{B}$

\begin{tabular}{|l|r|l|l|r|}
\hline & VITALITI & VitalPatch & Valedo & Opal \\
\hline Panel 1 & 2 & 4 & 1 & 3 \\
\hline Subgroup A & 2 & 4 & 1 & 3 \\
\hline Subgroup B & 2 & 4 & 1 & 3 \\
\hline
\end{tabular}

Rankings of the model alternatives remain unchanged with every subgroup compared to the complete panel 3 results. Valedo still has the highest importance ranking, followed by VITALITI, Opal and VitalPatch.

\subsubsection{Analysis of results with respect to expert disagreements in panel 4}

Experts in expert panel 4 disagree on the relative importance of organizational criteria in Organizational perspective. Hierarchical clustering analysis distinguished two subgroups in panel 4, which lowered the disagreement values. Table 65 below shows the relative importance of criteria in Organizational perspective. 
Table 65 Relative importance of criteria in expert panel 4 and its subgroups

\begin{tabular}{|c|c|c|c|c|}
\hline & $\begin{array}{l}\text { Training } \\
\text { Needed }\end{array}$ & Tech Support & Data Support & $\begin{array}{l}\text { Competitive } \\
\text { Advantage }\end{array}$ \\
\hline Panel 4 & 0.275 & 0.272 & 0.28 & 0.173 \\
\hline Subgroup A & 0.15 & 0.32 & 0.35 & 0.18 \\
\hline Subgroup B & 0.38 & 0.23 & 0.23 & 0.16 \\
\hline
\end{tabular}

The rankings of alternatives were recalculated according to results of 2 subgroups and compared to total rankings in panel 4 . Synthesis of priorities and rankings of alternatives with subgroups compared to overall panel 4 results are presented in Table 66.

Table 66 Global rankings of model alternatives of panel 4 and its subgroups A and $\mathrm{B}$

\begin{tabular}{|l|r|l|l|l|}
\hline & VITALITI & VitalPatch & Valedo & Opal \\
\hline Panel 1 & 2 & 4 & 1 & 3 \\
\hline Subgroup A & 2 & 4 & 1 & 3 \\
\hline Subgroup B & 2 & 4 & 1 & 3 \\
\hline
\end{tabular}

Rankings of the model alternatives remain unchanged with every subgroup compared to the complete panel 4 results. Valedo still has the highest importance ranking, followed by VITALITI, Opal and VitalPatch.

\subsubsection{Analysis of results with respect to expert disagreements in panel 5}

Experts in expert panel 5 had slight disagreements on the relative importance of patient criteria in Patient perspective. Hierarchical clustering analysis distinguished three subgroups in panel 5 , which lowered the 
disagreement values. Table 67 below shows the relative importance of criteria in Patient perspective.

Table 67 Relative importance of criteria in expert panel 5 and its subgroups

\begin{tabular}{|l|r|l|l|}
\hline & \multicolumn{2}{|l|}{ Patient Experience } & \multicolumn{2}{l|}{$\begin{array}{l}\text { Clinical Benefits for } \\
\text { Patients }\end{array}$} \\
\hline Panel 5 & 0.31 & 0.39 & 0.29 \\
\hline Subgroup A & 0.3 & 0.32 & 0.38 \\
\hline Subgroup B & 0.26 & 0.51 & 0.23 \\
\hline Subgroup C & 0.48 & 0.48 & 0.05 \\
\hline
\end{tabular}

The rankings of alternatives were recalculated according to results of 3 subgroups and compared to total rankings in panel 5 . Synthesis of priorities and rankings of alternatives with subgroups compared to overall panel 5 results are presented in Table 68.

Table 68 Global rankings of model alternatives of panel 5 and its subgroups A, B and $\mathrm{C}$

\begin{tabular}{|l|r|r|r|r|}
\hline & VITALITI & VitalPatch & Valedo & Opal \\
\hline Panel 1 & 2 & 4 & 1 & 3 \\
\hline Subgroup A & 2 & 4 & 1 & 3 \\
\hline Subgroup B & 2 & 4 & 1 & 3 \\
\hline Subgroup C & 2 & 4 & 1 & 3 \\
\hline
\end{tabular}

Rankings of the model alternatives remain unchanged with every subgroup compared to complete model calculations. Valedo still has the highest importance ranking, followed by VITALITI, Opal and VitalPatch. 


\subsubsection{Analysis of results with respect to expert disagreements in panel 6}

Experts in expert panel 6 had some disagreements on the relative importance of interpersonal criteria in Interpersonal perspective with respect to the mission. Hierarchical clustering analysis distinguished three subgroups in panel 6, which lowered the disagreement values. Table 69 shows the relative importance of criteria in Interpersonal perspective.

Table 69 Relative importance of criteria in expert panel 6 and its subgroups

\begin{tabular}{|c|c|c|c|}
\hline & $\begin{array}{l}\text { Efficiency of } \\
\text { Communication and } \\
\text { Patient Access }\end{array}$ & $\begin{array}{l}\text { Information } \\
\text { Sharing }\end{array}$ & $\begin{array}{l}\text { Educational Benefits and } \\
\text { New Knowledge Creation }\end{array}$ \\
\hline Panel 6 & 0.36 & 0.35 & 0.28 \\
\hline Subgroup A & 0.44 & 0.31 & 0.26 \\
\hline Subgroup B & 0.17 & 0.47 & 0.36 \\
\hline Subgroup C & 0.17 & 0.3 & 0.53 \\
\hline Subgroup D & 0.17 & 0.67 & 0.17 \\
\hline
\end{tabular}

The rankings of alternatives were recalculated according to results of 4 subgroups and compared to total rankings in panel 6 . Synthesis of priorities and rankings of alternatives with subgroups compared to overall panel 6 results are presented in Table 70. 
Table 70 Global rankings of model alternatives of panel 6 and subgroups A, B, C and D

\begin{tabular}{|l|r|r|r|r|}
\hline & VITALITI & VitalPatch & Valedo & Opal \\
\hline Panel 1 & 2 & 4 & 1 & 3 \\
\hline Subgroup A & 2 & 4 & 1 & 3 \\
\hline Subgroup B & 2 & 4 & 1 & 3 \\
\hline Subgroup C & 2 & 4 & 1 & 3 \\
\hline Subgroup D & 2 & 4 & 1 & 3 \\
\hline
\end{tabular}

Rankings of the model alternatives remain unchanged with every subgroup compared to complete model calculations. Valedo still has the highest importance ranking, followed by VITALITI, Opal and VitalPatch.

There were also slight disagreements in panels 7.1 .4 alternative rankings with respect to 'Decrease in hospital costs' criteria and 7.4.1 alternative rankings with respect to 'Patient experience' ranking criteria. When model results were recalculated and analyzed to account for disagreements, original rankings of model alternatives remain.

\subsection{Sensitivity Analysis}

Sensitivity analysis gives an opportunity to test the robustness of the model, test the tolerance ranges of the results and examine rankings of decision alternatives under different scenarios. Several kinds of sensitivity analyses are presented in this study.

1. Sensitivity analysis is conducted to determine the allowable range of perturbations of model perspectives that preserves the ranking of the top alternatives. 
2. Sensitivity analysis is carried out to determine the allowable range of perturbations in values of perspectives to preserve the ranking of all of the alternatives.

3. Sensitivity analysis of looking at changes in rankings of alternatives according to the different scenarios according to perspective focus.

Table 71 shows the rankings of the alternatives for the reference in the analysis presented below.

Table 71 Rankings and weights of model alternatives with respect to the mission

\begin{tabular}{|c|c|c|}
\hline Wearable Sensor Product & Rank & Weight \\
\hline Valedo & 1 & 0.2658 \\
\hline VITALITI & 2 & 0.2604 \\
\hline Opal & 3 & 0.2464 \\
\hline VitalPatch & 4 & 0.2274 \\
\hline
\end{tabular}

\subsubsection{HDM SA at the perspective level to preserve the ranking of the best alternative}

This analysis looks at allowable levels of perturbations - the threshold of changes to values of perspectives - to preserve the top ranking of Valedo (top wearable sensor product alternative). This type of sensitivity analysis also gives opportunity to look at the values of tolerance, operating sensitivity coefficients (OPSC) and total sensitivity coefficients (TSC). The values of conducted sensitivity analysis are displayed in Table 72 . 
Table 72 HDM SA at the perspectives level to preserve the ranking of the top alternative

\begin{tabular}{|c|c|c|c|c|c|}
\hline & Financial & Technical & Organizational & Patient & Interpersonal \\
\hline Base values & 0.203 & 0.227 & 0.173 & 0.248 & 0.149 \\
\hline $\begin{array}{l}\text { Allowable } \\
\text { ranges of } \\
\text { perturbations }\end{array}$ & $\begin{array}{c}{[-0.203} \\
0.114]\end{array}$ & $\begin{array}{c}{[-0.227} \\
0.773]\end{array}$ & {$[-0.173,0.313]$} & $\begin{array}{c}{[-0.061} \\
0.752]\end{array}$ & $\begin{array}{c}{[-0.149} \\
0.153]\end{array}$ \\
\hline Tolerance & {$[0,0.317]$} & {$[0,1]$} & {$[0,0.486]$} & {$[0.187,1]$} & {$[0,0.302]$} \\
\hline OPSC & $11.4 \%$ & $22.7 \%$ & $17.3 \%$ & $6.1 \%$ & $14.9 \%$ \\
\hline TSC & $31.7 \%$ & $100.0 \%$ & $48.6 \%$ & $81.3 \%$ & $30.2 \%$ \\
\hline
\end{tabular}

The results of the analysis show that Valedo will remain the top-ranking alternative if the model perspectives remain within their allowable levels of perturbation. Thus, if Financial perspective stays within [0, 0.317], the ranking of Valedo stays as top alternative or having a highest potential for technology adoption for pervasive care in neurosurgery and orthopedics. Any perturbations in Technical perspective preserve Valedo as top-ranking alternative.

Organizational perspective shows the allowable tolerance ranges of $[0,0.486]$. Changes in Patient perspective within $[0.187,1]$ will not shift the top-ranking alternative. Interpersonal perspective has a tolerance range of $[0,0.302]$ for keeping Valedo as a top-ranking alternative. Both Financial and Patient perspectives are the most critical perspectives with the lowest OPSC and TSC criteria. The probability that top alternative ranking will change when Patient perspectives changes uniformly between zero and one is $18.7 \%$. This analysis shows that model ranking solution is quite robust. 


\subsubsection{HDM SA at the perspective level to preserve the ranking of all alternatives}

This sensitivity analysis provides opportunity to look at the allowable levels of perturbations in model perspectives, which preserve the rankings of all alternatives. Table 73 below provides a detailed analysis of allowable ranges of perturbations, tolerance ranges, and coefficients: OPSC and TSC.

Table 73 HDM SA at the perspectives level aimed to preserve the ranking of all alternatives

\begin{tabular}{|l|c|c|c|c|r|}
\hline & Financial & Technical & Organizational & Patient & Interpersonal \\
\hline Base values & 0.203 & 0.227 & 0.173 & 0.248 & 0.149 \\
\hline $\begin{array}{l}\text { Allowable } \\
\text { ranges of } \\
\text { perturbations }\end{array}$ & $\begin{array}{c}{[-0.203,} \\
0.114]\end{array}$ & $\begin{array}{c}{[-0.227,} \\
0.578]\end{array}$ & {$[-0.173,0.313]$} & {$[-0.061,0.752]$} & $\begin{array}{c}{[-0.149,} \\
0.153]\end{array}$ \\
\hline Tolerance & {$[0,0.317]$} & {$[0,0.805]$} & {$[0,0.486]$} & {$[0.187,1]$} & {$[0,0.302]$} \\
\hline OPSC & $11.4 \%$ & $22.7 \%$ & $17.3 \%$ & $6.1 \%$ & $14.9 \%$ \\
\hline TSC & $31.7 \%$ & $80.5 \%$ & $48.6 \%$ & $81.3 \%$ & $30.2 \%$ \\
\hline
\end{tabular}

The most critical assessment perspective for preserving all the alternatives' ranks is Financial, since its OPSC $=11.4 \%$ as well as TSC $=31.7 \%$. The least sensitive perspectives is Technical perspective with tolerance range of $[0,0.805]$ and allowable ranges of perturbation of $[-0.227,0.578]$ from the base value of 0.227 . While OPSC of Interpersonal perspective has a low value of $14.9 \%$, its allowable range of perturbations is $[-0.149,0.153]$, which is $+/$ the base value of the Interpersonal perspective (0.149). The values of other perspectives are similar to the perturbations and tolerances of the sensitivity analysis to 
preserve the top alternative. As a result of that, the model could be considered reasonably robust.

\subsubsection{Scenario analysis}

Scenario analysis was conducted to understand the effects of changing relative importance of the technology assessment perspectives on rankings of model alternatives. Extreme weight variations were considered in "what-if" scenarios on perspectives while rankings of the model alternatives were observed. Since there are 5 main perspectives, five scenarios will be considered in this sensitivity analysis. In each case, one of the perspectives will be assigned a value of 0.96 , while four others will be assigned a value of 0.01 each (Table 74) (Abotah 2015; Iskin 2014). The scenarios might be useful if a clinic or hospital department wants to focus only on one of the model perspectives and look at the rankings of the alternatives under that main consideration (Table 75).

Table 74 Distribution of weights for model perspectives in five scenarios

\begin{tabular}{|l|r|r|r|r|r|}
\hline Perspectives & \multicolumn{1}{|c|}{ Financial } & \multicolumn{1}{|l|}{ Technical } & \multicolumn{1}{l|}{ Organizational } & \multicolumn{1}{l|}{ Patient } & \multicolumn{1}{|c|}{ Interpersonal } \\
\hline Original Values & 0.203 & 0.227 & 0.173 & 0.248 & 0.149 \\
\hline Scenario 1 & 0.96 & 0.01 & 0.01 & 0.01 & 0.01 \\
\hline Scenario 2 & 0.01 & 0.96 & 0.01 & 0.01 & 0.01 \\
\hline Scenario 3 & 0.01 & 0.01 & 0.96 & 0.01 & 0.01 \\
\hline Scenario 4 & 0.01 & 0.01 & 0.01 & 0.96 & 0.01 \\
\hline Scenario 5 & 0.01 & 0.01 & 0.01 & 0.01 & 0.96 \\
\hline
\end{tabular}


Table 75 Description of scenario focus

\begin{tabular}{|l|l|}
\hline Scenario & Description \\
\hline Financial focus & $\begin{array}{l}\text { The main point of focus in adoption of wearable sensor products by } \\
\text { neurosurgery and orthopedic departments is financial, which include } \\
\text { impacts of costs of acquisition, maintenance, savings from economies of } \\
\text { scale and decrease of overall hospital costs. }\end{array}$ \\
\hline Technical focus & $\begin{array}{l}\text { Focus on the technical aspect of adoption without much concern about } \\
\text { financial, organizational, patient and interpersonal criteria. Adopting from } \\
\text { the technical superiority standpoint. }\end{array}$ \\
\hline Organizational focus & $\begin{array}{l}\text { Mostly considering organizational aspects in adoption process, focusing on } \\
\text { training needed, tech and data support and competitive advantage. }\end{array}$ \\
\hline Patient focus & $\begin{array}{l}\text { Patient-driven adoption, where cost concerns or organizational, technical } \\
\text { and interpersonal concerns are practically not relevant. }\end{array}$ \\
\hline Interpersonal focus & $\begin{array}{l}\text { Interpersonal aspect is dominating the adoption focus with providing } \\
\text { communication and information sharing as well educational benefits. }\end{array}$ \\
\hline
\end{tabular}

Changes in global rankings of the alternatives according to each scenario are shown in figure below (Figure 47, Figure 48, Figure 49, Figure 50 and Figure 51).

\subsubsection{Financial focus scenario}

The main idea of this scenario is high consideration of financial criteria in adoption of wearable sensor products for pervasive care in neurosurgery and orthopedics. In this scenario the clinic is looking at the best possible financial adoption potential of wearable sensor products. 


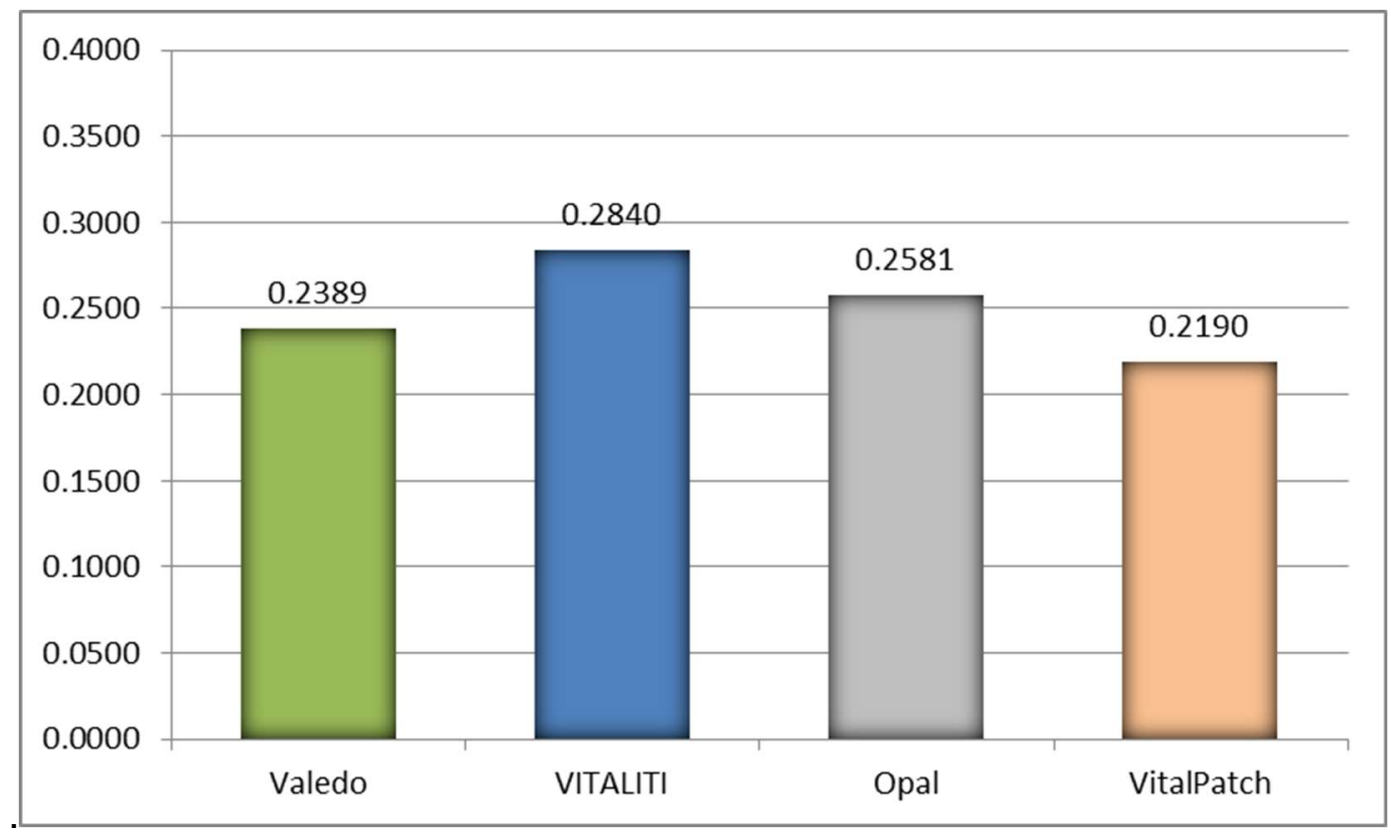

Figure 47 Global contribution of alternatives to the mission in scenario 1

According to the analysis (Figure 47) VITALITI (0.284) has the highest importance ranking in scenario 1 (Financial focus). Opal takes a second place in technology adoption according to financial focus scenario with the value of 0.2581. Valedo (0.2389), which ranked first under original considerations drops down to third place and VitalPatch remains fourth (0.2190).

\subsubsection{Technical focus scenario}

This scenario shows an extreme focus on the technical aspect of adoption without much concern about financial, organizational, patient and interpersonal perspectives. Adoption of wearable sensor devices is based solely from valuing technical adoption criteria. The results of calculations under this technical focus scenario are shown in Figure 48. 


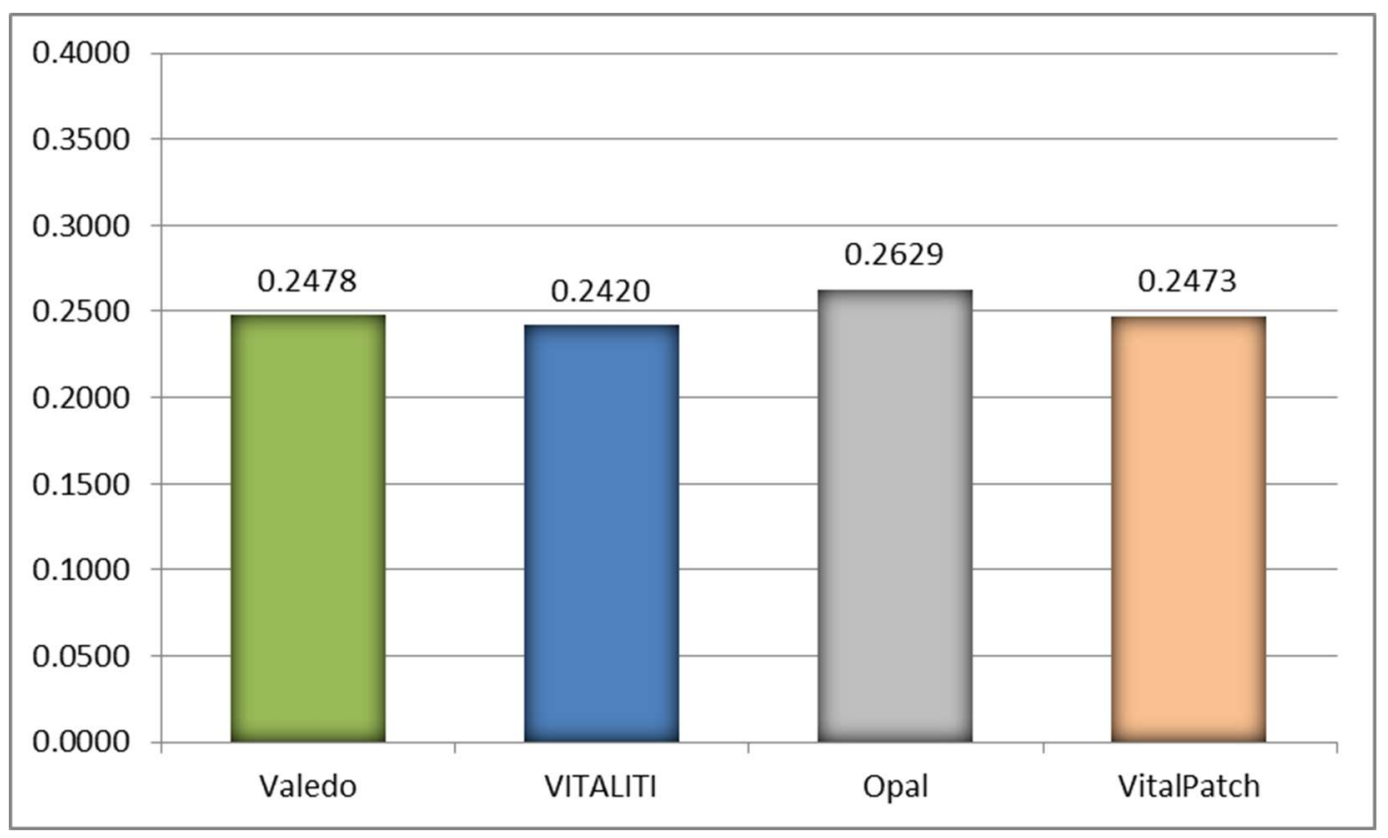

Figure 48 Global contribution of alternatives to the mission in scenario 2

According to the results, Opal rates the highest in this scenario with value of 0.2629. Opal is a research grade medical device, and is versatile. Valedo closely follows in second place (0.2478) and is almost tied with VitalPatch. VITALITI $(0.2420)$ has also very close values to top contenders. All devices are technologically advanced product solutions.

\subsubsection{Organizational focus scenario}

This scenario could be relevant for a clinic or hospital department that wants to consider the organizational impact of adoption of wearable medical devices. The main focus of the department would be on training needed, tech and data support and competitive advantage. The results of calculations under this organizational focus scenario are shown in Figure 49. 


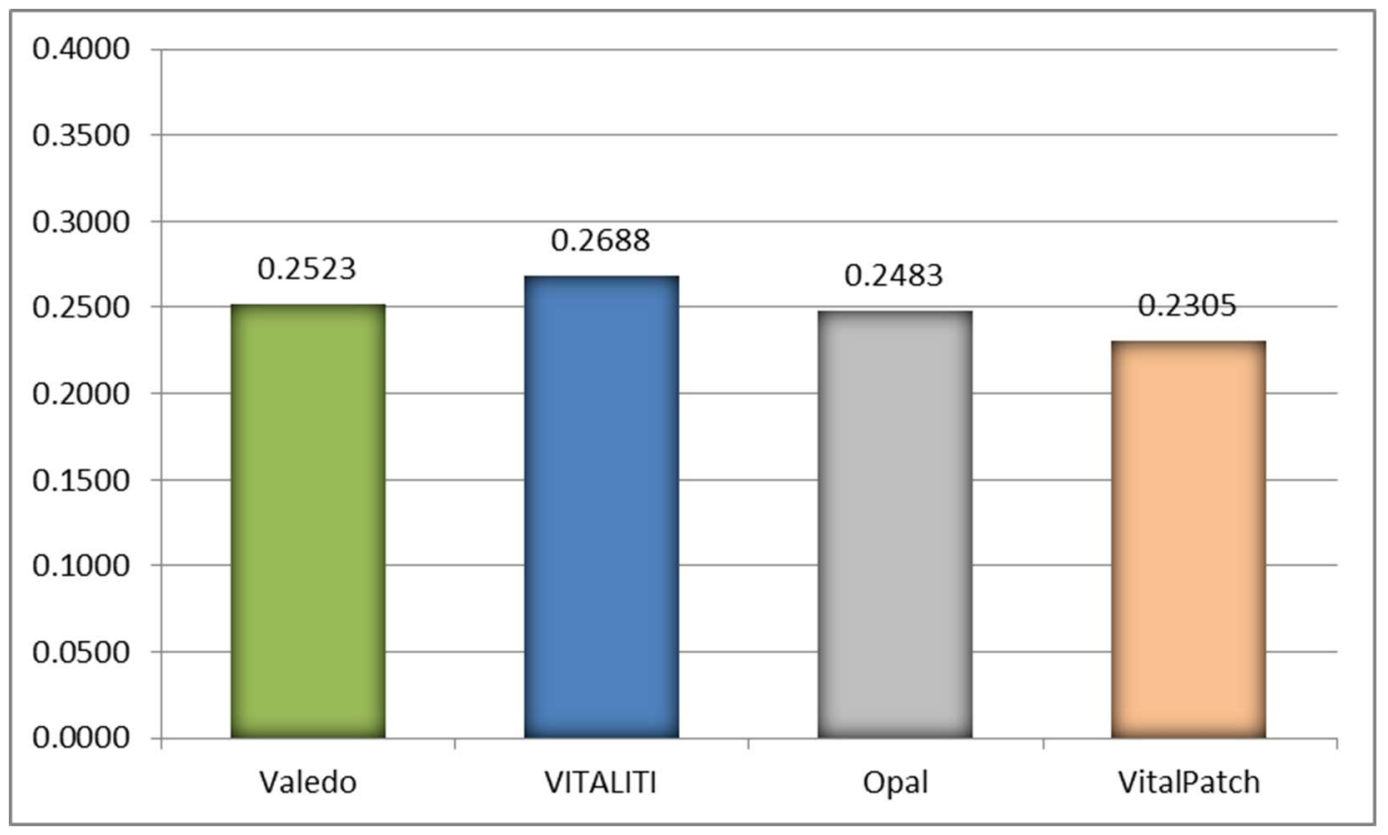

Figure 49 Global contribution of alternatives to the mission in scenario 3

The results of scenario 3 calculations show that VITALITI has the highest importance rating of 0.2688 . Valedo $(0.2523)$ is the second ranked alternative, followed by Opal (0.2483) and VitalPatch (0.2305).

\subsubsection{Patient focus scenario}

Scenario 4 is the case of extreme patient driven adoption, or just a way to look at the lineup of wearable sensor product consideration with a patientfocused lens. While first priority for doctors is well-being of patients, this scenario practically disregards other considerations: financial, organizational etc. The results of calculations under this patient focus scenario are shown in Figure 50. 


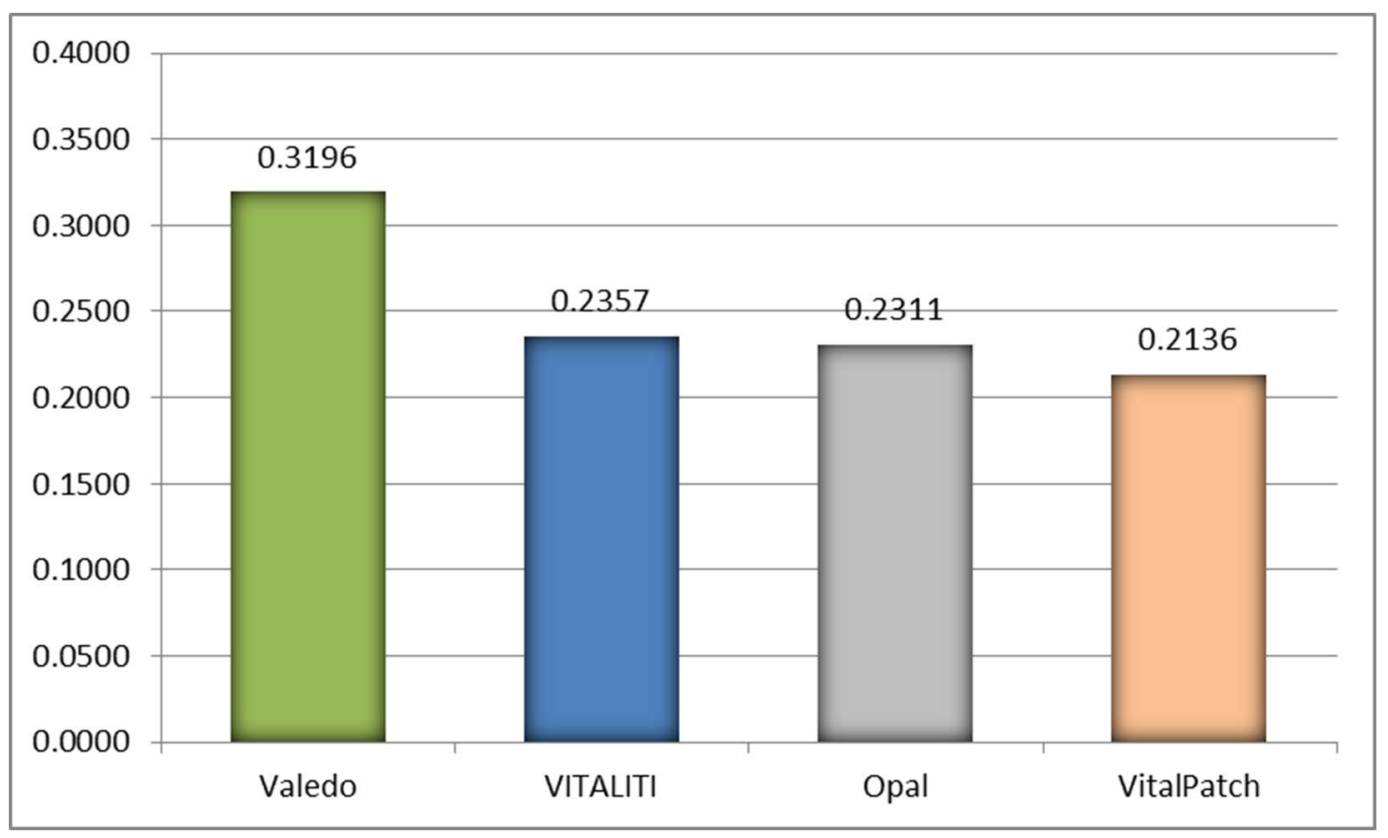

Figure 50 Global contribution of alternatives to the mission in scenario 4

According to the results, a clear top ranking solution is Valedo (0.3196).

The device engages patient encouragement tactics and has high rankings in patient experience criteria and clinical benefits for patients. VITALITI has the second importance ranking in technology adoption potential with 0.2357 . Opal (0.2311) and VitalPatch (0.2136) hold a third and fourth place respectively in this extreme patient perspective scenario.

\subsubsection{Interpersonal focus scenario}

This scenario focuses on Interpersonal perspective and its criteria of efficiency of communication and patient access, information sharing and educational benefits. If clinic is looking to improve its information sharing and communication channels through doctors, nurses and patients, it may was to 
look at the adoption of wearable medical products that can satisfy those goals. The results of calculations under this technical focus scenario are shown in Figure 51.

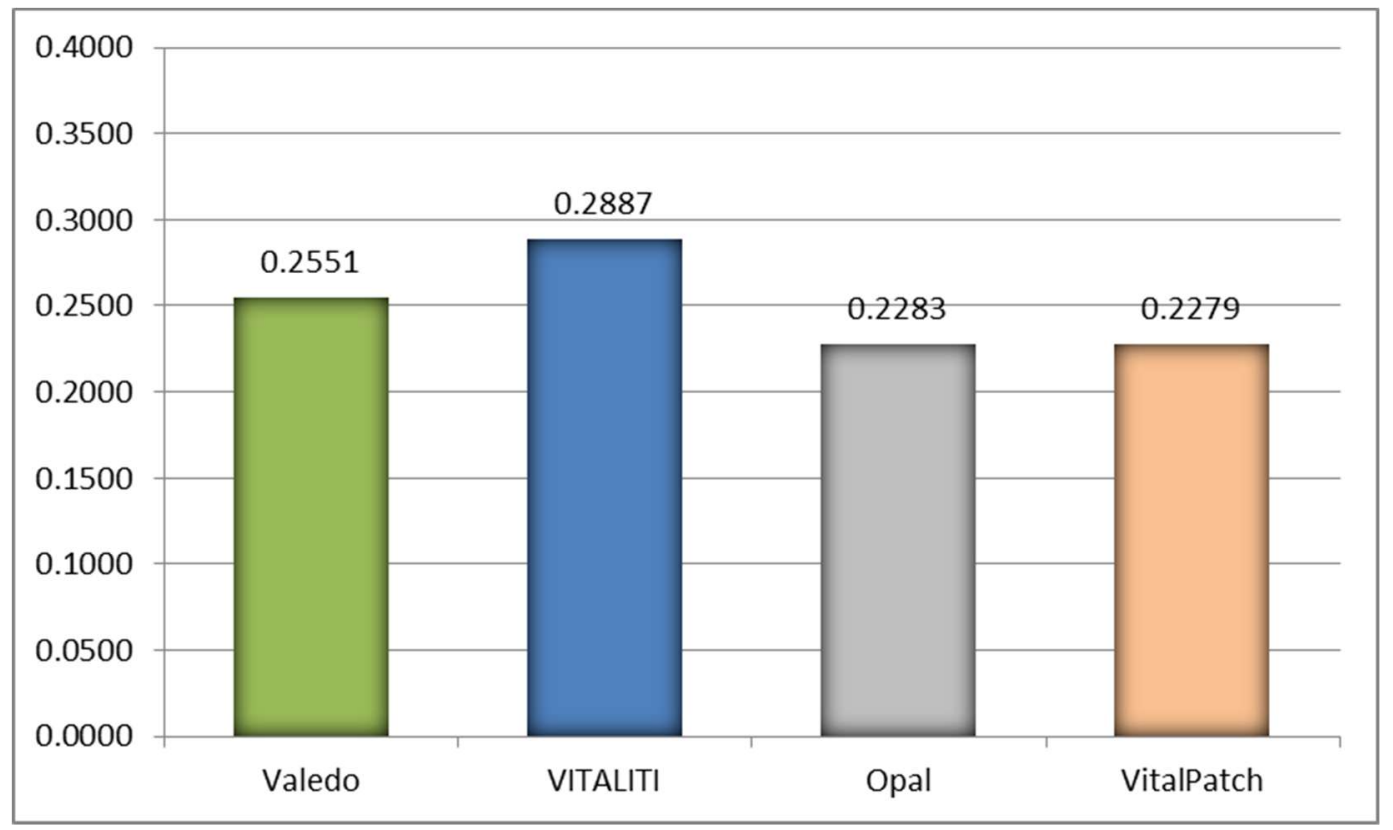

Figure 51 Global contribution of alternatives to the mission in scenario 5

VITALITI (0.2887) is a highly ranked alternative for technology adoption potential in this interpersonal-focus scenario. Valedo $(0.2551)$ is the second-best alternative in this case. Opal (0.2283) and VitalPatch (0.2279) have similar ranks with respect to the mission.

\subsection{Criterion-Related Validity}

Criterion-related validity tests whether the results of the model are valid, applicable and relatable in real-life evaluation. Experts were presented with the results of the research and asked to evaluate the appropriateness of the results 
obtained by the study and the appropriateness of generalizability of the model. The experts confirmed the validity of the model. 


\section{CHAPTER 9. DISCUSSIONS AND RECOMMENDATIONS}

This chapter discusses the results obtained from expert evaluations and the insights that those evaluations provide.

\subsection{Model Perspectives and Criteria}

Experts' panel results show that Patient perspective is the most important perspective to consider in case of technology adoption of wearable sensor technologies in neurosurgery and orthopedics. It is a leading perspective with $24.8 \%$ value. It is very important to note that results of the experts in the industry show that the main perspective while considering adoption of devices that are going to be used by patients is the one that includes the patient-centered criteria like clinical benefits for patients, patient experience and privacy and security. It seems only natural, that in healthcare industry patient benefits are placed first. This information could be used by the manufacturers of the devices, where they can emphasize patient benefits and experience as well as researchers that can explore patient perspective with the criteria in more detail.

Technical perspective with $22.7 \%$ weight is second highest ranked perspective for technology of wearable devices in neurosurgery and orthopedics. The importance of technical aspects in technology adoption and assessment in healthcare has been discussed widely in the literature and has been presented in the literature background chapter. Technical aspects of technology adoption are taken with great attention by the physicians and administrators in healthcare. The 
designers and manufacturers of the devices should consider the importance of technical criteria like reliability, ease of data access, physician's ease of use, usefulness and interoperability.

Financial aspects of technology adoption had the third highest ranking according to the experts in the study. This perspective is ranked in the top three, as it follows Patient and Technical perspective. Cost of acquisition and cost of maintenance are the most important criteria. Technology innovators need to know that Financial perspective is one of the most important criteria and will be influential in technology adoption decisions.

Organizational perspective is not in the top three, but still is an important perspective with $17.3 \%$ of overall weight. Technology trendsetters in the area of wearables for neurosurgery and orthopedics should understand that doctors and healthcare industry administrators gave criteria of 'Training needed', 'Tech support' and 'Data support' almost the same weight. So, there might be expectations that wearable products should have excellent tech support, data support and ability to provide training of the medical staff related to the innovative product.

Interpersonal perspective has the least importance according to the experts; however, with the result of $14.9 \%$, it ought to be noted that Interpersonal perspective should not be ignored. The future of the industry is in information sharing and efficiency of communication since it directly pertains to pervasiveness and ubiquitous nature of new technology direction. Perhaps 
makers of wearable sensor products can design and develop their innovations in the direction of improving communication among stakeholders of healthcare: patients, doctors, nurses and administrative staff. Neurosurgery and orthopedics departments should consider interpersonal aspects of adoption in their analysis of wearable devices.

\subsection{Wearable Sensor Products}

The results of the quantification of all the expert panels showed that Valedo has the highest potential for adoption with $26.58 \%$ weight, however, according to the results, alternatives obtained comparable competitive rankings. As discussed in Section 6.2.5. Research Focus, the model alternatives are medical-grade devices with superior and unique technical capabilities, backed by research studies and/or clinical trials suitable for pervasive care in neurosurgery and orthopedics department in the hospital or clinic. However, they have different designs and features. Valedo is a back pain therapy solution that features iOS/Android App with therapeutic exercises and provide feedback and motivation through tracking as well as sharing of the results. VITALITI is a close second place with the adoption potential weight of $26.04 \%$-- a medical device that rests on the neck, while being a part of a consumer/clinical vital sign platform with important mobility measure of step counted, posture monitor, slip and fall detector. Opal $(24.64 \%)$ is a system of sensors that measure activity level, tremor, intervention response, gait, balance, diurnal patterns and turning with streaming and logging data. Patch-based wearable device VitalPatch had the 
lowest score of $22.74 \%$, while it also provides comprehensive tracking, continuous monitoring and data analytics. All wearables received high ranking. The model is a research tool that shows the results of the evaluations by the experts involved in the study. It is not aimed to provide the answer to the general wearable adoption, but serve as guidance in technology adoption assessment.

Scenario analysis provides interesting view of how the certain perspective focus impacts the lineup of the wearable technology solutions. Thus, in financial focus scenario, VITALITI got the highest rank (28.4\%) and Opal had a second place (0.2581). From the Financial perspective view, the experts give preference to multiple component devices like VITALITI and Opal. In technical focus scenario, Opal is the highest alternative $(26.79 \%)$ with high scores of Reliability, Ease of Use, Usefulness, Interoperability and Data Access. Opal has advanced mobility focused technology and modular aspect. Organizational focus, with criteria like training needed, tech support, data support and competitive advantage, shows that VITALITI is a leading alternative (26.88\%). VITALITI also leads in Interpersonal focus scenario (28.87\%) according to the experts. VITALITI is an advanced tool that works as a part of the system for diagnosis of fifteen different conditions, with continuous streaming of various health parameters. According to the opinions of the experts in this study, VITALITI provides the highest potential for adoption considering solely organizational ecosystem benefit or exclusively interpersonal focus. Valedo has the first place $(31.96 \%)$ in technology adoption potential for Patient focus scenario. It is 
marketed as a medical device for digital back therapy at home and focused on patient experience, support, feedback and motivation.

When the designers and manufacturers emphasize benefits to patients and unique patient experience that their product provides, and it matches the adoption needs and goals of healthcare departments, it creates the winning product scenario for technology adoption. Higher attention to the patient needs, engagement and motivation shows attractiveness for adoption in healthcare. 


\section{CHAPTER 10. CONCLUSIONS, LIMITATIONS AND FUTURE RESEARCH}

\subsection{Assumptions and Limitations of the Proposed Research}

Since the proposed research engaged multiple expert panels consisting of experts from various fields (academia, engineering, healthcare, management), and they were asked to validate the results, the results may be impacted by subjectivity. Due to the nature of the hierarchical decision problem at hand, expert subjectivity may not be eliminated completely. The rigor of expert panels was increased by avoidance of dominant characters and unproductive discussions as well as equal and straightforward representation of different perspectives. This research also engaged various validity measures, like measurements of inconsistency and disagreement as well as sensitivity analysis to assess model rigor and robustness.

The research looked at the adoption problem from the physician's point of view, and with the majority of the evaluating experts in the case study involved in Oregon Health and Science University. Patients' viewpoint could give another interesting view on the problem of adoption of wearable devices and an interesting study to pursue.

Wearable technologies rapidly evolve; change and new kinds of wearable sensor products appear on the market. Clinics may be interested in assessing adoption of other wearable devices, and it's expected in ever-changing healthcare technology market. 
Research results are also context and time dependent and therefore could be generalized accordingly. Future possible governmental and insurance reimbursement opportunities for wearable could impact the framework of the model. Also, the drivers, impacting adoption decisions of wearable sensor product in neurosurgery and orthopedics, could change for internal or external reasons which may have an impact on objectives and goals as well as alternatives of the model.

Proposed research employed sensitivity analysis that looked at the impacts of potential scenarios according to possible changes in variables and for the purposes of improving generalization ability of the model. Scenario analysis looks at the extreme hypothetical scenarios, where the weight of one perspective is at the maximum and the rest of the perspectives receive minimum weight contributions, however other scenarios are possible and should be constructed according to the needs of the healthcare department assessing adoption.

\subsection{Contributions}

One of the main contributions of this research is the usage of HDM for healthcare technology assessment problem of technology adoption, in particular of assessment of technology adoption of wearable products in neurosurgery and orthopedics. The definition of a framework and assessment of the research perspectives and criteria through the judgment quantification shows the capabilities of hierarchical decision modeling in healthcare and enables solutions of similar problems in health care settings. 
This is was possible through achievement of the following objectives:

- Development of a hierarchical decision-making model (AHP/HDM) that incorporated important aspects and adoption needs of neurological surgery and orthopedics department with the possible alternative solutions;

- Validation of the model through the expert panel evaluations;

- Data collection through opinions of experts with the method of pairwise comparisons among all variables at every hierarchical level of the decision model;

- Analysis and interpretation of the data, with the possibility of generalization of the model for possible hospital-wide and nation-wide acceptance.

Other contributions of this research are presented below:

- Enabling healthcare professionals to look at the problem from multiple perspectives;

- Advancement in research of the perspectives and criteria central in health technology adoption assessment;

- Analysis of perspectives and their contribution to technology adoption of wearables in healthcare;

- The case study was focused on neurosurgery and orthopedics adoption of wearable sensor products;

- The study unveils that patient aspect dominates in expert opinions; 
- The hierarchical decision modeling software has been created with Ruby and $\mathrm{R}$ to make it available for researchers in an open source platform that could be updated and improved by the users;

- The learnings may lead to better incentives and programs for clinicians and help overcome certain barriers in their pervasive technology adoption;

- The model should provide a tool for evaluation of technology adoption potential of medical devices, which could lead to better understanding of technology adoption issues in healthcare, potentially improve clinician satisfaction with information technology and aid in betterment of quality of care.

The research is unique since it draws upon a vast literature review in healthcare information technologies, methodologies and technology adoption to select the perspectives and criteria of a technology adoption problem from physician's perspective for pervasive patient mobility care post-surgery in neurosurgery and orthopedics.

A broader, more complete view of the adoption problem will be established through the incorporation of multiple perspectives into the model for the benefit of healthcare professionals, wearables industry professionals and policy makers.

\subsection{Conclusions and Future Research}

Developed HDM model for assessment of technology adoption potential of wearable sensor products has a potential to be further implemented at OHSU 
and other clinics. It enables healthcare professionals to look at the problem from multiple perspectives. Learning which factors are significant may lead to better incentives and programs for clinicians and help overcome certain barriers in their healthcare technology implementations. This research may open new ways for the neurosurgery and orthopedics departments to strategize over their technology adoption potential. It reveals perceptions about adoption perspectives, criteria and wearable products in by healthcare industry, particularly in Pacific Northwest and OHSU. The framework could be tested by a particular department and with particular healthcare technology that has a possibility of adoption by that clinic/hospital department etc. The model should provide a tool for technology assessment in healthcare.

Further research could be done regarding at other types of health technology products, researching implementation of the model in private clinics, rural clinics or exploring health technology adoption from the patients' point of view. 


\section{REFERENCES}

Abbas, M., 2016. Consistency Analysis for Judgment Quantification in Hierarchical Decision Model, Ph.D. thesis, Portland State University.

Abotah, R., 2015. Evaluation of Energy Policy Instruments for the Adoption of Renewable Energy: Case of Wind Energy in the Pacific Northwest U.S., Ph.D. thesis, Portland State University.

Ackerman, K., 2011. When It Comes to EHR Adoption, Practice Size Matters. iHealthBeat 1.

Agarwal, A., 2002. Return on investment analysis for a computer-based patient record in the outpatient clinic setting. J. Assoc. Acad. Minor. Phys. 13, 61.

Aggelidis, V.P., Chatzoglou, P.D., 2009. Using a modified technology acceptance model in hospitals. Int. J. Med. Inform. 78, 115-26.

doi:10.1016/j.jjmedinf.2008.06.006

Ahn, M.J., Meeks, M.D., Davenport, S., Bednarek, R., 2009. Death of Distance? — Biotechnology Agglomeration Patterns, Alliance Proximity, and Firm Performance. Int. J. Innov. Technol. Manag. 6, 247-264. doi:10.1142/S0219877009001704 
Ahn, M.J., York, A.S., Sohn, S.Y., Benyamini, P., 2013. Biotechnology Innovation: a Legitimacy-Based View. Int. J. Innov. Technol. Manag. 10, 1350015. doi:10.1142/S0219877013500156

Ajzen, I., Madden, T.J., 1986. Prediction of goal-directed behavior: Attitudes, intentions, and perceived behavioral control. J. Exp. Soc. Psychol. 22, 453474. doi:10.1016/0022-1031(86)90045-4

Alkhateeb, F.M., Khanfar, N.M., Loudon, D., 2009. Physicians' Adoption of Pharmaceutical E-Detailing: Application of Rogers' Innovation-Diffusion Model. Serv. Mark. Q. 31, 116-132. doi:10.1080/15332960903408575

Alper, J., Olson, S., 2010. Report to the President Realizing the Full Potential of Health Information Technology to Improve Healthcare for Americans: the Path Forward.

Amer, M., Daim, T.U., 2011. Selection of renewable energy technologies for a developing county: A case of Pakistan. Energy Sustain. Dev. 15, 420-435. doi:10.1016/j.esd.2011.09.001

Ammenwerth, E., Brender, J., Nykänen, P., Prokosch, H.-U., Rigby, M., Talmon, J., 2004. Visions and strategies to improve evaluation of health information systems. Reflections and lessons based on the HIS-EVAL workshop in Innsbruck. Int. J. Med. Inform. 73, 479-91. doi:10.1016/j.jjmedinf.2004.04.004 
Anderson, T.R., Jahromi, S.R., 2016. Selecting Medical Hardware using Pairwise Comparisons: A Patient's Perspective of Cochlear Implant Device Selection, in: 2016 Proceedings of PICMET'16: Technology Management for Social Innovation. pp. 558-565.

André, B., Inger Ringdal, G., Loge, J.H., Rannestad, T., Laerum, H., Kaasa, S., 2008. Experiences with the Implementation of Computerized Tools in Health Care Units: A Review Article. Int. J. Hum. Comput. Interact. 24, 753-775. doi:10.1080/10447310802205768

Angst, C.M., Agarwal, R., Sambamurthy, V., Kelley, K., 2010. Social Contagion and Information Technology Diffusion: The Adoption of Electronic Medical Records in U.S. Hospitals. Manage. Sci. 56, 1219-1241.

doi:10.1287/mnsc.1100.1183

APDM, 2017. All day activity tracker and more - APDM wearable technologies [WWW Document]. URL https://www.apdm.com/continuous-recording/

Appelboom, G., Camacho, E., Abraham, M.E., Bruce, S.S., Dumont, E.L., Zacharia, B.E., D’Amico, R., Slomian, J., Reginster, J.Y., Bruyère, O., Connolly, E.S., 2014. Smart wearable body sensors for patient selfassessment and monitoring. Arch. public Heal. 72, 28. doi:10.1186/2049$3258-72-28$ 
Ash, J.S., Lyman, J., Carpenter, J., Fournier, L., 2001. A diffusion of innovations model of physician order entry. Proc. AMIA Symp. 22-6.

Ash, J., Bates, D., 2005. Factors and forces affecting EHR system adoption: report of a 2004 ACMI discussion. J. Am. Med. Informatics 12, 8-13. doi:10.1197/jamia.M1684.j

Atallah, L., Aziz, O., Gray, E., Lo, B., Yang, G.-Z., 2013. An Ear-Worn Sensor for the Detection of Gait Impairment After Abdominal Surgery. Surg. Innov. 20, 86-94. doi:10.1177/1553350612445639

Auvinen, H., Ruutu, S., Tuominen, A., Ahlqvist, T., Oksanen, J., 2014. Process supporting strategic decision-making in systemic transitions. Technol. Forecast. Soc. Change 1-18. doi:10.1016/j.techfore.2014.07.011

Ayatollahi, H., Bath, P. A., Goodacre, S., 2009. Paper-based versus computerbased records in the emergency department: staff preferences, expectations, and concerns. Health Informatics J. 15, 199-211. doi:10.1177/1460458209337433

Aziz, O., Atallah, L., Lo, B., Elhelw, M., Wang, L., Yang, G.Z., Darzi, a, 2007. A pervasive body sensor network for measuring postoperative recovery at home. Surg. Innov. 14, 83-90. doi:10.1177/1553350607302326 
Aziz, O., Atallah, L., Lo, B., Gray, E., Athanasiou, T., Darzi, A., Yang, G.-Z., 2011. Ear-worn body sensor network device: an objective tool for functional postoperative home recovery monitoring. J Am Med Inf. Assoc 18, 156-159. doi:10.1136/jamia.2010.005173

Azzone, G., Manzini, R., 2008. Quick and dirty technology assessment: The case of an Italian Research Centre. Technol. Forecast. Soc. Change 75, 13241338. doi:10.1016/j.techfore.2007.10.004

Bächlin, M., Plotnik, M., Roggen, D., Maidan, I., Hausdorff, J.M., Giladi, N., Tröster, G., 2010. Wearable assistant for Parkinsons disease patients with the freezing of gait symptom. IEEE Trans. Inf. Technol. Biomed. 14, 436446. doi:10.1109/TITB.2009.2036165

Baker, P.M.A., Gandy, M., Zeagler, C., 2015. Innovation and Wearable Computing: A Proposed Collaborative Policy Design Framework. IEEE Internet Comput. 19, 18-25. doi:10.1109/MIC.2015.74

Banerjee, A., Verma, S., Bagade, P., Gupta, S.K.S., 2012. Health-Dev: Model Based Development Pervasive Health Monitoring Systems. 2012 9th Int. Conf. Wearable Implant. Body Sens. Networks 85-90.

doi:10.1109/BSN.2012.33

Banos, O., Villalonga, C., Damas, M., Gloesekoetter, P., Pomares, H., Rojas, I., 2014. PhysioDroid: Combining Wearable Health Sensors and Mobile 
Devices for a Ubiquitous, Continuous, and Personal Monitoring. Sci. World J. 2014, 1-11.

Banta, D., 1988a. Technology assessment and new kidney stone treatment methods by F. Kamper-Jørgensen, S. Challah and T. Folmer Anderson (Eds.). Health Policy (New. York). 10, 119-120.

Banta, D., 1988b. New medical devices: invention, development, and use; by K.Ekelman (Ed.). Health Policy (New. York). 11, 277-278.

Banta, D., 1991. National priorities for the assessment of clinical conditions and medical technologies. Report of a Pilot Study. Health Policy (New. York). 17, 310.

Banta, D., Bos, M., 1991. The relation between quantity and quality with coronary artery bypass graft (CABG) surgery. Health Policy (New. York). 18, 1-10.

Banta, D., 2003. The development of health technology assessment. Health Policy (New. York). 63, 121-132.

Banta, H.D., 1990. Medical technology in China. Health Policy (New. York). 14, $127-137$.

Banta, H.D., Gelijns, A., Griffioen, J., Graaff, P., 1987. An inquiry concerning future health care technology: methods and general results. Health Policy (New. York). 8, 251-264. 
Banta, H., 1994. Health care technology as a policy issue. Health Policy (New. York). 30, 1-21.

Banta, H., 1993. The cost-effectiveness of 10 selected applications in Minimally Invasive Therapy. Health Policy (New. York). 23, 135-151.

Banta, H., Schersten, T., Jonsson, E., 1993. Implications of minimally invasive therapy. Health Policy (New. York). 23, 167-178.

Banta, H., Vondeling, H., 1993. Diffusion of minimally invasive therapy in Europe. Health Policy (New. York). 23, 125-133.

Baram, Y., Lenger, R., 2012. Gait Improvement in Patients with Cerebral Palsy by Visual and Auditory Feedback. Neuromodulation Technol. Neural Interface 15, 48-52. doi:10.1111/j.1525-1403.2011.00412.x

Başoğlu, A.N., Daim, T.Ü., Işkin, T., Aydinoğlu, B., Öztürk, M., 2012. New product development for the healthcare industry: A case study of diet software. Heal. Policy Technol. 1, 93-104.

Basoglu, N., 2007. Organizational adoption of enterprise resource planning systems: A conceptual framework. J. High Technol. Manag. Res. 18, 73-97.

Basoglu, N., Daim, T.U., Topacan, U., 2012. Determining Patient Preferences for Remote Monitoring. J. Med. Syst. 36, 1389-1401. doi:10.1007/s10916-010$9601-1$ 
Bates, D.W., Leape, L.L., Cullen, D.J., Laird, N., Petersen, L. A., Teich, J.M., Burdick, E., Hickey, M., Kleefield, S., Shea, B., Vander Vliet, M., Seger, D.L., 1998. Effect of computerized physician order entry and a team intervention on prevention of serious medication errors. JAMA 280, 1311-6.

Bates, D.W., Ebell, M., Gotlieb, E., Zapp, J., Mullins, H.C., 2003. A Proposal for Electronic Medical Records in U.S. Primary Care. J. Am. Informatics Assoc. 10, 1-10. doi:10.1197/jamia.M1097.

Battista, R., Banta, H., Jonnson, E., 1994. Lessons from the eight countries. Health Policy (New. York). 30, 397-421.

Becker, A., Herzberg, D., Marsden, N., Thomanek, S., Jung, H., Leonhardt, C., 2011. A new computer-based counselling system for the promotion of physical activity in patients with chronic diseases--results from a pilot study. Patient Educ. Couns. 83, 195-202. doi:10.1016/j.pec.2010.05.024

Beckett, M., Quiter, E., Ryan, G., Berrebi, C., Taylor, S., Cho, M., Pincus, H., Kahn, K., 2011. Bridging the gap between basic science and clinical practice: The role of organizations in addressing clinician barriers. Implement. Sci. 6, 35. doi:10.1186/1748-5908-6-35

Beekum, T. van, Banta, H., 1989. Possibilities and problems in the development of home care technology. Health Policy (New. York). 12, 301-307. 
Behkami, N.A., 2012. Examining Health Information Technology Implementations using Structural and Behavioral Capabilities: Case of The Patient-Centered Medical Home, Ph.D. thesis, Portland State University.

Behkami, N. A., U. Daim, T., 2012. Research forecasting for health information technology (HIT), using technology intelligence. Technol. Forecast. Soc. Change 79, 498-508. doi:10.1016/j.techfore.2011.08.015

Bentley, S.M., Melville, J.L., Berry, B.D., Katon, W.J., 2007. Implementing a clinical and research registry in obstetrics: Overcoming the barriers. Gen. Hosp. Psychiatry 29, 192-8. doi:10.1016/j.genhosppsych.2007.01.011

Bergman-Evans, B., Kuhnel, L., McNitt, D., Myers, S., 2008. Uncovering beliefs and barriers: staff attitudes related to advance directives. Am. J. Hosp. Palliat. Care 25, 347-53. doi:10.1177/1049909108320883

Bhattacharya, N., 2015. Biometrics in Mobile Devices is the Key to the Future.

Bianco, V., Scarpa, F., Tagliafico, L. a., 2014. Scenario analysis of nonresidential natural gas consumption in Italy. Appl. Energy 113, 392-403. doi:10.1016/j.apenergy.2013.07.054

Bierbooms, J.J.P.A., Bongers, I.M.B., van Oers, H. a M., 2011. A scenario analysis of the future residential requirements for people with mental health 
problems in Eindhoven. BMC Med. Inform. Decis. Mak. 11, 1. doi:10.1186/1472-6947-11-1

Blumenthal, D., 2009. Stimulating the adoption of health information technology. N. Engl. J. Med. 360, 1477-1479.

Blumenthal, D., 2010. Launching HITECH. N. Engl. J. Med. 362, 382-5. doi:10.1056/NEJMp0912825

Bodenheimer, T., Grumbach, K., 2003. Electronic technology a spark to revitalize primary care? JAMA 290, 259-264.

Bohanec, M., Zupan, B., Rajkovic, V., 2000. Applications of qualitative multiattribute decision models in health care. Int. J. Med. Inform. 58-59, 191205. doi:10.1016/S1386-5056(00)00087-3

Boonstra, A., Broekhuis, M., 2010. Barriers to the acceptance of electronic medical records by physicians from systematic review to taxonomy and interventions. BMC Health Serv. Res. 10, 231. doi:10.1186/1472-6963-10231

Bowens, F.M., Frye, P. A., Jones, W. A, 2010. Health information technology: integration of clinical workflow into meaningful use of electronic health records. Perspect. Health Inf. Manag. 7, 1d. 
Box, T.L., McDonell, M., Helfrich, C.D., Jesse, R.L., Fihn, S.D., Rumsfeld, J.S., 2010. Strategies from a nationwide health information technology implementation: the VA CART story. J. Gen. Intern. Med. 25 Suppl 1, 72-6. doi:10.1007/s11606-009-1130-6

Brand, C., Landgren, F., Hutchinson, A., Jones, C., Macgregor, L., Campbell, D., 2005. Clinical practice guidelines: barriers to durability after effective early implementation. Intern. Med. J. 35, 162-9. doi:10.1111/j.14455994.2004.00763.x

Brent, A.C., 2012. Technology assessment in developing countries: sustainable energy systems in the African context. Int. J. Innov. Technol. Manag. 9, 1250035. doi:10.1142/S0219877012500356

Brown, D.E., 2008. Introduction to data mining for medical informatics. Clin. Lab. Med. 28, 9-35, v. doi:10.1016/j.cll.2007.10.008

Bruce, B., Fries, J., 2005. The Arthritis, Rheumatism and Aging Medical Information System (ARAMIS)-Still young at 30 years. Clin. Exp. Rheumatol. 23, S163-167.

Bufalino, V.J., Masoudi, F. A., Stranne, S.K., Horton, K., Albert, N.M., Beam, C., Bonow, R.O., Davenport, R.L.V., Girgus, M., Fonarow, G.C., Krumholz, H.M., Legnini, M.W., Lewis, W.R., Nichol, G., Peterson, E.D., Rumsfeld, J.S., Schwamm, L.H., Shahian, D.M., Spertus, J. A., Woodard, P.K., Yancy, 
C.W., 2011. The American Heart Association's recommendations for expanding the applications of existing and future clinical registries: a policy statement from the American Heart Association., Circulation.

doi:10.1161/CIR.0b013e3182181529

Burton-Jones, A., Hubona, G.S., 2006. The mediation of external variables in the technology acceptance model. Inf. Manag. 43, 706-717. doi:10.1016/j.im.2006.03.007

Buytendijk, F., Hatch, T., Micheli, P., 2010. Scenario-based strategy maps. Bus. Horiz. 53, 335-347. doi:10.1016/j.bushor.2010.02.002

Carayon, P., Cartmill, R., Blosky, M.A., Brown, R., Hackenberg, M., Hoonakker, P., Hundt, A.S., Norfolk, E., Wetterneck, T.B., Walker, J.M., 2011. ICU nurses' acceptance of electronic health records. J. Am. Med. Inform. Assoc. 18, 812-9. doi:10.1136/amiajnl-2010-000018

Carvalho, M.M., Fleury, A., Lopes, A.P., 2013. An overview of the literature on technology roadmapping (TRM): Contributions and trends. Technol. Forecast. Soc. Chang. 80, 1418-1437.

Chan, K.-Y. A., Oerlemans, L. A. G., Pretorius, M.W., 2010. Knowledge exchange behaviours of science park firms: the innovation hub case. Technol. Anal. Strateg. Manag. 22, 207-228. doi:10.1080/09537320903498546 
Chan, L., Daim, T., Kocaoglu, D., 2013. Developing a Strategic Policy Choice Framework for Technological Innovation: A Case Study on the Biopharmaceutical Industry in China, in: 2013 Proceedings of PICMET'13: Technology Management for Emerging Technologies. pp. 474-486.

Chau, P.Y.K., Hu, P.J.-H., 2002. Investigating healthcare professionals' decisions to accept telemedicine technology: an empirical test of competing theories. Inf. Manag. 39, 297-311. doi:10.1016/S0378-7206(01)00098-2

Chaudhry, B., Wang, J., Wu, S., Maglione, M., Mojica, W., Roth, E., Morton, S., Shekelle, P., 2006. Systematic Review : Impact of Health Information Technology on Quality, Efficiency, and Costs of Medical Care. Ann. Intern. Med. 144, 742-752, NaN-168-NaN-185.

Chen, H., Ho, J.C., Kocaoglu, D.F., 2009. A Strategic Technology Planning Framework: A Case of Taiwan's Semiconductor Foundry Industry. IEEE Trans. Eng. Manag. 56, 4-15. doi:10.1109/TEM.2008.927815

Chen, H., Li, J., 2011. A sensitivity analysis algorithm for the constant sum pairwise comparison judgments in hierarchical decision models, in: Portland International Conference on Management of Engineering and Technology. pp. 1050-1066. 
Chen, H., Kocaoglu, D.F., 2008. A sensitivity analysis algorithm for hierarchical decision models. European Journal of Operational Research, 185, pp. 266288.

Chen, R.-F., Hsiao, J.-L., 2012. An investigation on physicians' acceptance of hospital information systems: A case study. Int. J. Med. Inform. 1-11. doi:10.1016/j.jjmedinf.2012.05.003

Chen, Y.-P., Hsieh, S.-H., Cheng, P.-H., Chien, T.-N., Chen, H.-S., Luh, J.-J., Lai, J.-S., Lai, F., Chen, S.-J., 2010. An agile enterprise regulation architecture for health information security management. Telemed. J. E. Health. 16, 807-17. doi:10.1089/tmj.2010.0023

Cheng, Y.-M., 2012. Exploring the roles of interaction and flow in explaining nurses' e-learning acceptance. Nurse Educ. Today. doi:10.1016/j.nedt.2012.02.005

Cherry, B., 2006. Determining facilitators and barriers to adoption of electronic health records in long-term care facilities. UMI Dissertation Services, ProQuest Information and Learning, Ann Arbor, MI.

Chiasson, M., Reddy, M., Kaplan, B., Davidson, E., 2007. Expanding multidisciplinary approaches to healthcare information technologies: what does information systems offer medical informatics? Int. J. Med. Inform. 76 Suppl 1, S89-97. doi:10.1016/j.jimedinf.2006.05.010 
Cho, I., Kim, J., Kim, J.H., Kim, H.Y., Kim, Y., 2010. Design and implementation of a standards-based interoperable clinical decision support architecture in the context of the Korean EHR. Int. J. Med. Inform. 79, 611-622. doi:10.1016/j.jmedinf.2010.06.002

Choi, Y.K., Totten, J.W., 2012. Self-construal's role in mobile TV acceptance: Extension of TAM across cultures. J. Bus. Res. 65, 1525-1533. doi:10.1016/j.jbusres.2011.02.036

Chow, M., Chan, L., Lo, B., Chu, W.-P., Chan, T., Lai, Y.-M., 2012a. Exploring the intention to use a clinical imaging portal for enhancing healthcare education. Nurse Educ. Today 1-8. doi:10.1016/j.nedt.2012.01.009

Chow, M., Herold, D.K., Choo, T.-M., Chan, K., 2012b. Extending the technology acceptance model to explore the intention to use Second Life for enhancing healthcare education. Comput. Educ. 59, 1136-1144. doi:10.1016/j.compedu.2012.05.011

Chumbler, N.R., Haggstrom, D., Saleem, J.J., 2011. Implementation of health information technology in Veterans Health Administration to support transformational change: telehealth and personal health records. Med. Care 49 Suppl, S36-42. doi:10.1097/MLR.0b013e3181d558f9

Ciuti, G., Ricotti, L., Menciassi, A., Dario, P., 2015. MEMS sensor technologies for human centred applications in healthcare, physical activities, safety and 
environmental sensing: A review on research activities in Italy. Sensors (Switzerland) 15, 6441-6468. doi:10.3390/s150306441

Cleland, D., Kocaoglu, D., 1981. Engineering Management. McGraw-Hill.

Clifton, L., Clifton, D. A., Pimentel, M. A. F., Watkinson, P.J., Tarassenko, L., 2013. Gaussian processes for personalized e-health monitoring with wearable sensors. IEEE Trans. Biomed. Eng. 60, 193-197. doi:10.1109/TBME.2012.2208459

Cloud DX, 2017. VITALITI - Qualcomm tricoder XPRIZE entry [WWW Document]. URL https://www.clouddx.com/\#/vitaliti

CMS, 2014. National Health Expenditure Projections 2014 - 2024.

Coates, J., 1974. Some methods and techniques for comprehensive impact assessment. Technol. Forecast. Soc. Change 6, 341-357.

Condell, J., Curran, K., Quigley, T., Gardiner, P., McNeill, M., Winder, J., Xie, E., Qi, Z., Connolly, J., 2011. Finger movement measurements in arthritic patients using wearable sensor enabled gloves. Int. J. Hum. Factors Model. Simul. 2, 276. doi:10.1504/IJHFMS.2011.045000

Cooper, G.F., 1986. A diagnostic method that uses causal knowledge and linear programming in the application of Bayes' formula. Comput. Methods Programs Biomed. 22, 223-37. 
CRAN - Package AHP [WWW Document], 2017. URL https://cran.rproject.org/web/packages/ahp/index.html

Cresswell, K., Sheikh, A., 2012. Organizational issues in the implementation and adoption of health information technology innovations: An interpretative review. Int. J. Med. Inform. doi:10.1016/j.jjmedinf.2012.10.007

Crocker, P., Lewis, M., 2010. Mobile Phone Sensors and Sensory Interfaces: Market Analysis and Forecasts.

Cunningham, S.W., van der Lei, T.E., 2009. Decision-making for new technology: A multi-actor, multi-objective method. Technol. Forecast. Soc. Change 76, 26-38. doi:10.1016/j.techfore.2008.08.012

Cyranoski, D., 2012. Tsunami simulations scare Japan. Nature 484, 296-297.

Daim, T., Basoglu, N., Tanoglu, I., 2010. A critical assessment of information technology adoption: technical, organizational and personal perspectives. Int. J. Bus. Inf. Syst. 6, 315-335.

Daim, T., Li, X., Kim, J., Simms, S., 2012a. Evaluation of energy storage technologies for integration with renewable electricity: Quantifying expert opinions. Environ. Innov. Soc. Transitions 3, 29-49. 
Daim, T.U., Bayraktaroglu, E., Estep, J., Lim, D.J., Upadhyay, J., Yang, J., 2012b. Optimizing the NW off-shore wind turbine design. Math. Comput. Model. 55, 396-404. doi:10.1016/j.mcm.2011.08.018

Daim, T.U., Bhatla, A., Mansour, M., 2013. Site selection for a data centre - a multi-criteria decision-making model. Int. J. Sustain. Eng. 6, 10-22. doi:10.1080/19397038.2012.719554

Daim, T.U., Kocaoglu, D.F., 2008. Exploring technology acquisition in Oregon, Turkey and in the U.S. electronics manufacturing companies. J. High Technol. Manag. Res. 19, 45-58. doi:10.1016/j.hitech.2008.06.007

Daim, T.U., Oliver, T., 2008. Implementing technology roadmap process in the energy services sector: A case study of a government agency. Technol. Forecast. Soc. Change 75, 687-720. doi:10.1016/j.techfore.2007.04.006

Daim, T.U., Rueda, G., Martin, H., Gerdsri, P., 2006. Forecasting emerging technologies: Use of bibliometrics and patent analysis. Technol. Forecast. Soc. Change 73 (8), 981-1012.

Daim, T., Gerdsri, N., Kockan, I., Kocaoglu, D., 2011. Technology Development Envelope Approach for The Adoption of Future Powertrain Technologies: A Case Study on Ford Otosan Roadmapping Model. J. Transp. Syst. Eng. Inf. Technol. 11, 58-69. doi:10.1016/S1570-6672(10)60112-2 
Davidson, S., Heineke, J., 2007. Toward an effective strategy for the diffusion and use of clinical information systems. J. Am. Med. ... 14, 361-367. doi:10.1197/jamia.M2254.Introduction

Davis, F.D., 1985. A technology acceptance model for empirically testing new end-user information systems: Theory and results. Management. Massachusetts Institute of Technology, Sloan School of Management.

Davis, F., 1989. User acceptance of computer technology: a comparison of two theoretical models. Manage. Sci. 35, 982-1003.

Davis, F., 1993. User acceptance of information technology: system characteristics, user perceptions and behavioral impacts. Int. J. Man. Mach. Stud. $38,475-487$.

Davis, F.D., Venkatesh, V., 1996. A critical assessment of potential measurement biases in the technology acceptance model: three experiments. Int. J. Hum. Comput. Stud. 45, 19-45. doi:10.1006/ijhc.1996.0040

Degoulet, P., Jean, F.C., Safran, C., 1995. The health care professional multimedia workstation: development and integration issues. Int. J. Biomed. Comput. 39, 119-25. 
DeLia, D., Hall, A., Prinz, T., Billings, J., 2004. What matters to low-income patients in ambulatory care facilities? Med. Care Res. Rev. 61, 352-75. doi:10.1177/1077558703257850

DeLone, W.H., McLean, E.R., 2003. The DeLone and McLean Model of Information Systems Success: A Ten-Year Update. J. Manag. Inf. Syst. 19, 9-30.

DePhillips, H., 2007. Initiatives and Barriers to Adopting Health Information Technology: A US Perspective. Dis. Manag. Heal. Outcomes 15, 1-6.

Despont-Gros, C., Mueller, H., Lovis, C., 2005. Evaluating user interactions with clinical information systems: A model based on human - computer interaction models. J. Biomed. Inform. 38, 244-255. doi:10.1016/j.jbi.2004.12.004

DesRoches, C.M., Campbell, E.G., R.Rao, S., Donelan, K., 2008. Electronic Health Records in Ambulatory Care - A National Survey of Physicians. N. Engl. J. Med. 359, 50-60.

Devlin, N.J., Sussex, J., 2011. Incorporating Multiple Criteria in HTA: Methods and Processes, Health Economics.

Dexter, P.R., Perkins, S.M., Maharry, K.S., Jones, K., McDonald, C.J., 2004. Inpatient computer-based standing orders vs physician reminders to 
increase influenza and pneumococcal vaccination rates: a randomized trial. JAMA 292, 2366-71. doi:10.1001/jama.292.19.2366

Dhawan, A.P., Heetderks, W.J., Pavel, M., Acharya, S., Akay, M., Mairal, A., Wheeler, B., Dacso, C.C., 2015. Current and Future Challenges in Point-ofCare Technologies : A Paradigm-Shift in Affordable Global Healthcare With Personalized and Preventive Medicine. IEEE J. Transl. Eng. Heal. Med. 3.

Diaby, V., Campbell, K., Goeree, R., 2013. Multi-criteria decision analysis (MCDA) in health care: A bibliometric analysis. Oper. Res. Heal. Care 2, 20 24. doi:10.1016/j.orhc.2013.03.001

Dillon, A., Morris, M.G., 1996. User acceptance of new information technology theories and models. M. Williams Annu. Rev. Inf. Sci. Technol. 31, 3-32.

Dixon, B.E., Zafar, A., Overhage, J.M., 2010. A Framework for evaluating the costs, effort, and value of nationwide health information exchange. J. Am. Med. Inform. Assoc. 17, 295-301. doi:10.1136/jamia.2009.000570

Dixon, D.R., 1999. The behavioral side of information technology. Int. J. Med. Inform. 56, 117-23.

Duda, R.O., Hart, P.E., Stork, D.G., 2001. Pattern classification. Wiley.

Dulaney, K., Redman, P., Clark, W., Ghubril, A.C., Hung, M., 2011. Predicts 2012: Mobile and Wireless Technologies Rise, Gartner. 
Dulcic, Z., Pavlic, D., Silic, I., 2012. Evaluating the Intended Use of Decision Support System (DSS) by Applying Technology Acceptance Model (TAM) in Business Organizations in Croatia. Procedia - Soc. Behav. Sci. 58, 15651575. doi:10.1016/j.sbspro.2012.09.1143

Dunbrack, L.A., 2014. U. S. Connected Health 2014 Top 10 Predictions : The New Care Delivery Model.

Dünnebeil, S., Sunyaev, A., Blohm, I., Leimeister, J.M., Krcmar, H., 2012. Determinants of physicians' technology acceptance for e-health in ambulatory care. Int. J. Med. Inform. 81, 746-60. doi:10.1016/j.jjmedinf.2012.02.002

Elwyn, G., Edwards, A., Kinnersley, P., Grol, R., 2000. Shared decision making and the concept of equipoise: the competences of involving patients in healthcare choices. Br. J. Gen. Pract. 50, 892-9.

Escayola, J., Martínez, I., Trigo, J., García, J., Martínez-Espronceda, M., Led, S., Serrano, L., 2009. Recent innovative advances in biomedical engineering: Standard-based design for ubiquitous p-Health. 4th Int. Multi-Conference Comput. Glob. Inf. Technol. ICCGI 2009 109-117.

doi:10.1109/ICCGI.2009.24 
Farra, N., El-Sayed, B., Moacdieh, N., Hajj, H., Hajj, Z., Haidar, R., 2011. A mobile sensing and imaging system for real-time monitoring of spine health. J. Med. Imaging Heal. Informatics 1, 238-245. doi:10.1166/jmihi.2011.1034

Feied, C.F., Smith, M.S., Handler, J. A., 2004. Keynote address: medical informatics and emergency medicine. Acad. Emerg. Med. 11, 1118-26. doi:10.1197/j.aem.2004.08.022

Fensli, R., Pedersen, P.E., Gundersen, T., Hejlesen, O., 2008. Sensor acceptance model - Measuring patient acceptance of wearable sensors. Methods Inf. Med. 47, 89-95. doi:10.3414/ME9106

Fenwick, D., Daim, T.U., Gerdsri, N., 2009. Technological Forecasting \& Social Change Value Driven Technology Road Mapping ( VTRM ) process integrating decision making and marketing tools : Case of Internet security technologies. Technol. Forecast. Soc. Chang. 76, 1055-1077. doi:10.1016/j.techfore.2009.04.005

Ferng, J.-J., 2009. Applying input-output analysis to scenario analysis of ecological footprints. Ecol. Econ. 69, 345-354. doi:10.1016/j.ecolecon.2009.08.006

Figueira, José \& Mousseau, Vincent \& Roy, Bernard \& Greco, Salvatore \& Ehrogott, Matthias. (2005). Electre Methods. International Series in 
Operations Research and Management Science. 78. 133-153. 10.1007/0387-23081-5_4.

Fleischer, T., Decker, M., Fiedeler, U., 2005. Assessing emerging technologiesMethodological challenges and the case of nanotechnologies. Technol. Forecast. Soc. Change 72, 1112-1121. doi:10.1016/j.techfore.2004.10.005

Folland, S., 2006. Health Care in Small Areas of Three Command Economies: What Do the Data Tell Us? East. Europ. Econ. 43, 31-52. doi:10.2753/EEE0012-8755430602

Fonkych, K., Taylor, R., 2005. The state and pattern of health information technology adoption. RAND Corporation., pp. 1-52.

Ford, E., Menachemi, N., Phillips, T., 2006. Predicting the adoption of electronic health records by physicians: When will health care be paperless? J. Am. Med. Inf. Assoc 13, 106-113. doi:10.1197/jamia.M1913.slow

Förster, B., Von der Gracht, H., 2014. Assessing Delphi panel composition for strategic foresight - A comparison of panels based on company-internal and external participants. Technol. Forecast. Soc. Change 84, 215-229. doi:10.1016/j.techfore.2013.07.012 
Frambach, R.T., Schillewaert, N., 2002. Organizational innovation adoption: a multi-level framework of determinants and opportunities for future research. J. Bus. Res. 55, 163-176. doi:10.1016/S0148-2963(00)00152-1

Furukawa, M.F., 2011. Electronic medical records and the efficiency of hospital emergency departments. Med. Care Res. Rev. 68, 75-95. doi:10.1177/1077558710372108

Gagnon, R.J., 1991. Assessing strategies for obtaining advanced engineering technologies with highly uncertain benefits. IEEE Trans. Eng. Manag. 38, 210-223. doi:10.1109/17.83754

Gerdsri, N., 2005. An Analytycal Approach on Building a Technology Development Envelope (TDE) for Roadmapping of Emerging Technologies, Ph.D. thesis, Portland State University. doi:10.1177/001088048102200214

Gerdsri, P., Kocaoglu, D., 2009. A Systematic Approach to Developing 1ational Technology Policy and Strategy for Emerging Technologies: A Case Study of 1anotechnology for Thailand's Agriculture Industry, in: PICMET 2009 Proceedings. pp. 447-461.

Gerdsri, P., Kocaoglu, D.F., 2008. HDM for Developing National Emerging Technology Strategy and Policy Supporting Sustainable Economy: A Case Study of Nanotechnology for Thailand's Agriculture, in: PICMET '08 - 2008 
Portland International Conference on Management of Engineering \& Technology. pp. 27-31.

Gerdsri, P., Kocaoglu, D.F., 2013. Evaluating Nanotechnologies for the Development of National Agriculture Industry. Int. J. Innov. Technol. Manag. 10, 1340004. doi:10.1142/S021987701340004X

Geum, Y., Lee, S., Kang, D., Park, Y., 2011. Technology roadmapping for technology-based product-service integration: A case study. J. Eng. Technol. Manag. 28, 128-146. doi:10.1016/j.jengtecman.2011.03.002

Girosi, F., Meili, R., Scoville, R., 2005. Extrapolating Evidence of Health Information Technology Savings and Costs, Pub. no. MG-410. Santa Monica, California.

Glaser, J., Henley, D., Downing, G., Brinner, K., 2008. Advancing Personalized Health Care through Health Information Technology: An Update from the American Health Information Community's Personalized Health Care Workgroup. J. Am. Med. Informatics Assoc. 15, 391-396. doi:10.1197/jamia.M2718.Setting

Glickman, S.W., Kit Delgado, M., Hirshon, J.M., Hollander, J.E., Iwashyna, T.J., Jacobs, A.K., Kilaru, A.S., Lorch, S. A., Mutter, R.L., Myers, S.R., Owens, P.L., Phelan, M.P., Pines, J.M., Seymour, C.W., Ewen Wang, N., Branas, C.C., 2010. Defining and measuring successful emergency care networks: a 
research agenda. Acad. Emerg. Med. 17, 1297-305. doi:10.1111/j.15532712.2010.00930.x

Goetghebeur, M.M., Wagner, M., Khoury, H., Levitt, R.J., Erickson, L.J., Rindress, D., 2012. Bridging Health Technology Assessment (HTA) and Efficient Health Care Decision Making with Multicriteria Decision Analysis (MCDA). Med. Decis. Mak. 32, 376-388. doi:10.1177/0272989X11416870

Goldberg, D.G., 2012. Primary care in the United States: the practice-based innovations and factors that influence adoption. J. Health Organ. Manag. 26, 81-97. doi:10.1108/14777261211211106

Goldzweig, C.L., Towfigh, A., Maglione, M., Shekelle, P.G., 2009. Costs and benefits of health information technology: new trends from the literature. Health Aff. (Millwood). 28, w282-93. doi:10.1377/hlthaff.28.2.w282

Gorla, N., Somers, T.M., Wong, B., 2010. Organizational impact of system quality, information quality, and service quality. J. Strateg. Inf. Syst. 19, 207228. doi:10.1016/j.jsis.2010.05.001

Goroll, A.H., Simon, S.R., Tripathi, M., Ascenzo, C., Bates, D.W., 2008. Community-wide implementation of health information technology: the Massachusetts eHealth Collaborative experience. J. Am. Med. Inform. Assoc. 16, 132-9. doi:10.1197/jamia.M2899 
Greenberger, M., Crenson, M.A., Crissev, B.L., 1976. Models in the Policy Process, Russet Sage Foundation, New York, NY, USA

Greenes, R., 2007. Clinical Decision Support: The Road Ahead. Amsterdam: Elsevier Academic Press.

Greenhalgh, T., Stramer, K., Bratan, T., Byrne, E., Mohammad, Y., Russell, J., 2008. Introduction of shared electronic records: multi-site case study using diffusion of innovation theory. Bmj 337, a1786-a1786. doi:10.1136/bmj.a1786

Greenhalgh, T., Potts, H.W.W., Wong, G., Bark, P., Swinglehurst, D., 2009. Tensions and Paradoxes in Electronic Patient Record Research: A Systematic Literature Review Using the Meta-narrative Method. Milbank Q. $87,729-788$.

Greenspun, H., 2012. Issue Brief : Physician perspectives about health information technology. pp.1-8.

Grupp, H., Hohmeyer, O., 1986. A technometric model for the assessment of technological standards and their application to selected technologyintensive products. Technol. Forecast. Soc. Change 30, 123-137. doi:10.1016/0040-1625(86)90015-6 
Guennoun, M., Zandi, M., El-Khatib, K., 2008. On the Use of Biometrics to Secure Wireless Biosensor Networks. 2008 3rd Int. Conf. Inf. Commun. Technol. From Theory to Appl. 1-5. doi:10.1109/ICTTA.2008.4530273

Gurses, A., Xiao, Y., 2006. A Systematic Review of the Literature on Multidisciplinary Rounds to Design Information Technology. J. Am. Med. Informatics Assoc. 13, 267-276. doi:10.1197/jamia.M1992.Communication

Hagger, M.S., Anderson, M., Kyriakaki, M., Darkings, S., 2007. Aspects of identity and their influence on intentional behavior: Comparing effects for three health behaviors. Pers. Individ. Dif. 42, 355-367. doi:10.1016/j.paid.2006.07.017

Hahn, K.A., Ohman-Strickland, P.A., Cohen, D.J., Piasecki, A.K., Crosson, J.C., Clark, E.C., Crabtree, B.F., 2011. Electronic medical records are not associated with improved documentation in community primary care practices. Am. J. Med. Qual. 26, 272-7. doi:10.1177/1062860610392365

Handy, J., Hunter, I., Whiddett, R., 2001. User acceptance of inter-organizational electronic medical records. Health Informatics J. 7, 103-107. doi:10.1177/146045820100700208

Haron, S.N., Hamida, M.Y., Talib, A., 2012. Towards Healthcare Service Quality: An Understanding of the Usability Concept in Healthcare Design. Procedia Soc. Behav. Sci. 42, 63-73. doi:10.1016/j.sbspro.2012.04.167 
Hastie, T., Tibshirani, R., Friedman, J., 2009. The Elements of Statistical Learning: Data Mining, Inference, and Prediction, 2nd ed. Springer.

Hatton, J.D., Schmidt, T.M., Jelen, J., 2012. Adoption of Electronic Health Care Records: Physician Heuristics and Hesitancy. Procedia Technol. 5, 706715. doi:10.1016/j.protcy.2012.09.078

Hayez, Q., Mareschal, B., De Smet, Y., 2009. New GAIA visualization methods. Proc. Int. Conf. Inf. Vis. 247-251. doi:10.1109/IV.2009.15

Helfrich, C.D., Savitz, L. A., Swiger, K.D., Weiner, B.J., 2007. Adoption and implementation of mandated diabetes registries by community health centers. Am. J. Prev. Med. 33, S50-8-65. doi:10.1016/j.amepre.2007.04.002

Helms, E., Williams, L., 2011. Evaluating Access Control of Open Source Electronic Health Record Systems, in: SEHC'11, May 22-23, 2011, Waikiki, Honolulu, HI, USA. pp. 63-70.

Henriksen, A.D.P., 1997. A technology assessment primer for management of technology. Int. J. Technol. Manag. 13, 615. doi:10.1504/IJTM.1997.001681

Hocoma, 2017. Medical Device for back therapy at home - Valedo [WWW Document]. 
Holden, R.J., Karsh, B.-T., 2010. The technology acceptance model: its past and its future in health care. J. Biomed. Inform. 43, 159-72. doi:10.1016/j.jbi.2009.07.002

Howard, R.A., 2006. The Foundations of DA Revisited, in: Advances: Foundations of DA Revisited. p. 44.

Howard, R.A., 1966. Decision Analysis: Applied Decision Theory, in: Proceedings of the 4th International Conference on Operational Research. pp. 55-77.

Hoyle, R.H., 2012. Introduction and Overview, in: Hoyle, R.H. (Ed.), Handbook of Structural Equation Modeling. The Guilford Press, New York, pp. 3-16.

Hundy, B., Hamblin, D., 1988. Risk and assessment of investment in new technology. Int. J. Prod. ... 26, 1799-1810.

Hung, S.-Y., Ku, Y.-C., Chien, J.-C., 2012. Understanding physicians' acceptance of the Medline system for practicing evidence-based medicine: a decomposed TPB model. Int. J. Med. Inform. 81, 130-42. doi:10.1016/j.jjmedinf.2011.09.009

Hyde, K., Maier, H.R., Colby, C., 2003. Incorporating uncertainty in the PROMETHEE MCDA method. J. Multi-Criteria Decis. Anal. 12, 245-259. doi:10.1002/mcda.361 Y3 - 26.02.2013 U6 - 
http://onlinelibrary.wiley.com/store/10.1002/mcda.361/asset/361_ftp.pdf?v=1

\&t=hdn7d86x\&s=302904a1acf9a17132814275398d236d8502d2f1

Ideker, K., Todicheeney-Mannes, D., Kim, S.C., 2011. A confirmatory study of violence risk assessment tool (M55) and demographic predictors of patient violence. J. Adv. Nurs. 67, 2455-62. doi:10.1111/j.1365-2648.2011.05667.x

Im, I., Kim, Y., Han, H.-J., 2008. The effects of perceived risk and technology type on users' acceptance of technologies. Inf. Manag. 45, 1-9. doi:10.1016/j.im.2007.03.005

Intille, S.S., 2004. A new research challenge: persuasive technology to motivate healthy aging. IEEE Trans. Inf. Technol. Biomed. 8, 235-237. doi:10.1109/TITB.2004.835531

Iskin, I., 2014. An Assessment Model for Energy Efficiency Program Planning in Electric Utilities: Case of the Pacific of Northwest U.S.A., Ph.D. thesis, Portland State University.

Iskin, I., Daim, T.U., 2014. Technology Assessment for Energy Efficiency Programs in Pacific Northwest, in: 2014 Proceedings of PICMET'14: Infrastructure and Service Integration. pp. 498-506.

Jackson, D.L., 2001. Structural Equation Modeling: A Sample Size and Number of Parameter Estimates in Maximum Likelihood Confirmatory Factor 
Analysis: A Monte Carlo Investigation. Struct. Equ. Model. A Multidiscip. J. 8, 205-223. doi:10.1207/S15328007SEM0802

Jackson, D.L., 2003. Structural Equation Modeling : A Revisiting Sample Size and Number of Parameter Estimates: Some Support for the $\mathrm{N}$ : $\mathrm{q}$ Hypothesis. Struct. Equ. Model. A Multidiscip. J. 10, 128-141. doi:10.1207/S15328007SEM1001

Janczewski, L., Shi, F.X., 2002. Development of information security baselines for healthcare information systems in New Zealand. Comput. Secur. 21, 172-192.

Jang, W.-J., Lee, C., 2013. a Technology Valuation Model for the Defense R\&D With Income Approach. Int. J. Innov. Technol. Manag. 10, 1350017. doi:10.1142/S021987701350017X

Jeng, D.J.-F., Tzeng, G.-H., 2012. Social influence on the use of Clinical Decision Support Systems: Revisiting the Unified Theory of Acceptance and Use of Technology by the fuzzy DEMATEL technique. Comput. Ind. Eng. 62, 819-828. doi:10.1016/j.cie.2011.12.016

Jian, W.-S., Syed-Abdul, S., Sood, S.P., Lee, P., Hsu, M.-H., Ho, C.-H., Li, Y.-C., Wen, H.-C., 2012. Factors influencing consumer adoption of USB-based Personal Health Records in Taiwan. BMC Health Serv. Res. 12, 277. doi:10.1186/1472-6963-12-277 
Jimoh, L., Pate, M. A., Lin, L., Schulman, K. A., 2012. A model for the adoption of ICT by health workers in Africa. Int. J. Med. Inform. 81, 773-81. doi:10.1016/j.jjmedinf.2012.08.005

Johnson, M.P., Zheng, K., Padman, R., 2012. Modeling the longitudinality of user acceptance of technology with an evidence-adaptive clinical decision support system. Decis. Support Syst. doi:10.1016/j.dss.2012.10.049

Jolly, D.R., 2008. Chinese vs. European views regarding technology assessment: Convergent or divergent? Technovation 28, 818-830. doi:10.1016/j.technovation.2008.09.001

Jonsson, E., Banta, H., 1994. Health care technology in Sweden. Health Policy (New. York). 30, 257-294.

Jung, S., 2006. The Perceived Benefits of Healthcare Information Technology Adoption: Construct and Survey Development., M.S. thesis, Louisiana State University.

Kang, D.O., Lee, H.J., Ko, E.J., Kang, K., Lee, J., 2006. A wearable context aware system for ubiquitous healthcare. Annu. Int. Conf. IEEE Eng. Med. Biol. - Proc. 5192-5195. doi:10.1109/IEMBS.2006.259538 
Karahanna, E., Straub, D.W., 1999. The psychological origins of perceived usefulness and ease-of-use. Inf. Manag. 35, 237-250. doi:10.1016/S03787206(98)00096-2

Karvonen, M., Lehtovaara, M., Kässi, T., 2012. Build-Up of Understanding of Technological Convergence: Evidence From Printed Intelligence Industry. Int. J. Innov. Technol. Manag. 9, 1250020. doi:10.1142/S0219877012500204

Kazemzadeh, R., Sartipi, K., Jayaratna, P., 2010. A Framework for Data and Mined Knowledge Interoperability in Clinical Decision Support Systems. Int. J. Healthc. Inf. Syst. Informatics 5, 37-60.

Kazley, A.S., Ozcan, Y. A., 2008. Do hospitals with electronic medical records (EMRs) provide higher quality care?: an examination of three clinical conditions. Med. Care Res. Rev. 65, 496-513.

doi:10.1177/1077558707313437

Kazley, A.S., Ozcan, Y. A., 2007. Organizational and Environmental Determinants of Hospital EMR Adoption: A National Study. J. Med. Syst. 31, 375-384. doi:10.1007/s10916-007-9079-7

Keller, P., Ledergerber, U., 1998. Bimodal System Dynamic A Technology Assessment and Forecasting Approach. Technol. Forecast. Soc. Change 58, 47-52. doi:10.1016/S0040-1625(97)00054-1 
Kerimoglu, O., Basoglu, N., Daim, T., 2008. Organizational adoption of information technologies: Case of enterprise resource planning systems. J. High Technol. Manag. Res. 19, 21-35. doi:10.1016/j.hitech.2008.06.002

Khoumbati, K., Themistocleous, M., Irani, Z., 2006. Evaluating the Adoption of Enterprise Application Integration in Health-Care Organizations. J. Manag. Inf. Syst. 22, 69-108. doi:10.2753/MIS0742-1222220404

Kim, D., Chang, H., 2006. Key functional characteristics in designing and operating health information websites for user satisfaction: an application of the extended technology acceptance model. Int. J. Med. Inform. 76, 790800. doi:10.1016/j.ijmedinf.2006.09.001

Kim, S., Malhotra, N., 2005. A longitudinal model of continued IS use: An integrative view of four mechanisms underlying postadoption phenomena. Manage. Sci. 51, 741-755.

King, W.R., He, J., 2006. A meta-analysis of the technology acceptance model. Inf. Manag. 43, 740-755. doi:10.1016/j.im.2006.05.003

Kirste, T., Hoffmeyer, A., Koldrack, P., Bauer, A., Schubert, S., Schröder, S., Teipel, S., 2014. Detecting the effect of Alzheimer's disease on everyday motion behavior. J. Alzheimers. Dis. 38, 121. doi:10.3233/JAD-130272 
Klingbeil, L., Reiner, R., Romanovas, M., Traechtler, M., Manoli, Y., 2010. Multimodal sensor data and information fusion for localization in indoor environments. Position. Navig. Commun. (WPNC), 2010 7th Work. 187-192. doi:10.1109/WPNC.2010.5654128

Kocaoglu, D.F., 1983. A participative approach to program evaluation. IEEE Trans. Eng. Manag. 30, 30.

Kodali, R., Mishra, R.P., Anand, G., 2009. Justification of world-class maintenance systems using analytic hierarchy constant sum method. J. Qual. Maint. Eng. 15, 47-77. doi:10.1108/13552510910943886

Köhler, A.R., Som, C., 2014. Risk preventative innovation strategies for emerging technologies the cases of nano-textiles and smart textiles. Technovation 34, 420-430. doi:http://dx.doi.org/10.1016/j.technovation.2013.07.002

Kukafka, R., Johnson, S.B., Linfante, A., Allegrante, J.P., 2003. Grounding a new information technology implementation framework in behavioral science: a systematic analysis of the literature on IT use. J. Biomed. Inform. 36, 218227. doi:10.1016/j.jbi.2003.09.002

Kulendran, M., Lim, M., Laws, G., Chow, A., Nehme, J., Darzi, A., Purkayastha, S., 2014. Surgical Smartphone Applications Across Different Platforms: Their Evolution, Uses, and Users. Surg. Innov. 21, 427-440. doi:10.1177/1553350614525670 
Kumar, S., Aldrich, K., 2010. Overcoming barriers to electronic medical record (EMR) implementation in the US healthcare system: A comparative study. Health Informatics J. 16, 306-18. doi:10.1177/1460458210380523

Kuo, Y.-L., Culhane, K.M., Thomason, P., Tirosh, O., Baker, R., 2009. Measuring distance walked and step count in children with cerebral palsy: An evaluation of two portable activity monitors. Gait \&amp; Posture 29, 304-310. doi:10.1016/j.gaitpost.2008.09.014

Labys, W., 1982. Measuring the validity and performance of energy models. Energy Econ. 4, 159-168. doi:10.1016/0140-9883(82)90015-9

Lane, N.D., Miluzzo, E., Lu, H., Peebles, D., Choudhury, T., Campbell, A.T., 2010. A survey of mobile phone sensing. Commun. Mag. IEEE 48, 140-150. doi:10.1109/MCOM.2010.5560598

Lanham, H.J., Leykum, L.K., McDaniel, R.R., 2012. Same organization, same electronic health records (EHRs) system, different use: exploring the linkage between practice member communication patterns and EHR use patterns in an ambulatory care setting. J. Am. Med. Inform. Assoc. 19, 382-391. doi:10.1136/amiajnl-2011-000263

Lapinsky, S.E., Holt, D., Hallett, D., Abdolell, M., Adhikari, N.K.J., 2008. Survey of information technology in Intensive Care Units in Ontario, Canada. BMC Med. Inform. Decis. Mak. 8, 5. doi:10.1186/1472-6947-8-5 
Lazoi, M., Ceci, F., Corallo, A., Secundo, G., 2011. Collaboration in an Aerospace SMEs Cluster: Innovation and ICT Dynamics. Int. J. Innov. Technol. Manag. 8, 393-414. doi:10.1142/S0219877011002398

Ledley, R.S., Lusted, L.B., 1959. Reasoning Foundations of Medical Diagnosis: Symbolic logic, probability, and value theory aid our understanding of how physicians reason. Science (80-. ). 130, 9-21.

doi:10.1126/science.130.3366.9

Lee, G., Xia, W., 2011. A longitudinal experimental study on the interaction effects of persuasion quality, user training, and first-hand use on user perceptions of new information technology. Inf. Manag. 48, 288-295. doi:10.1016/j.im.2011.09.003

Lee, J.H., Kim, H., Phaal, R., 2012. An analysis of factors improving technology roadmap credibility: A communications theory assessment of roadmapping processes. Technol. Forecast. Soc. Change 79, 263-280.

doi:10.1016/j.techfore.2011.05.003

Lee, K.-J., Ohta, T., Kakehi, K., 2010. Formal boundary spanning by industry liaison offices and the changing pattern of university-industry cooperative research: the case of the University of Tokyo. Technol. Anal. Strateg.

Manag. 22, 189-206. doi:10.1080/09537320903498538 
Légaré, F., Ratté, S., Stacey, D., Kryworuchko, J., Gravel, K., Id, G., Turcotte, S., 2010. Interventions for improving the adoption of shared decision making by healthcare professionals (Review). Cochrane Database of Systematic Reviews, 4(CD006732).

Legris, P., Ingham, J., Collerette, P., 2003. Why do people use information technology? A critical review of the technology acceptance model. Inf. Manag. 40, 191-204. doi:10.1016/S0378-7206(01)00143-4

Leu, M.G., Cheung, M., Webster, T.R., Curry, L., Bradley, E.H., Fifield, J., Burstin, H., 2008. Centers speak up: the clinical context for health information technology in the ambulatory care setting. J. Gen. Intern. Med. 23, 372-8. doi:10.1007/s11606-007-0488-6

Liang, H., Xue, Y., Chase, S.K., 2011. Online health information seeking by people with physical disabilities due to neurological conditions. Int. J. Med. Inform. 80, 745-53. doi:10.1016/j.ijmedinf.2011.08.003

Liao, A., Hull, C.E., Sriramachandramurthy, R., 2013. the Six Facets Model of Technology Management: a Study in the Digital Business Industry. Int. J. Innov. Technol. Manag. 10, 1350019. doi:10.1142/S0219877013500193

Lim, S., Xue, L., Yen, C.C., Chang, L., Chan, H.C., Tai, B.C., Duh, H.B.L., Choolani, M., 2011. A study on Singaporean women's acceptance of using 
mobile phones to seek health information. Int. J. Med. Inform. 80, e189-202. doi:10.1016/j.jjmedinf.2011.08.007

Linder, J. A., Ma, J., Bates, D.W., Middleton, B., Stafford, R.S., 2007. Electronic health record use and the quality of ambulatory care in the United States. Arch. Intern. Med. 167, 1400-5. doi:10.1001/archinte.167.13.1400

Linder, S.H., Sexton, K., 2011. Conceptual models for cumulative risk assessment. Am. J. Public Health 101 Suppl, S74-81. doi:10.2105/AJPH.2011.300318

Linstone, H. A., Lendaris, G.G., Rogers, S.D., Wakeland, W., Williams, M., 1979. The use of structural modeling for technology assessment. Technol. Forecast. Soc. Change 14, 291-327. doi:10.1016/0040-1625(79)90032-5

Liu, L., Ma, Q., 2005. The impact of service level on the acceptance of application service oriented medical records. Inf. Manag. 42, 1121-1135. doi:10.1016/j.im.2004.12.004

Liu, Y., Guo, H., Zhang, Z., Wang, L., Dai, Y., Fan, Y., 2007. An optimization method based on scenario analysis for watershed management under uncertainty. Environ. Manage. 39, 678-90. doi:10.1007/s00267-006-0029-9

Liyanage, S., 1995. Breeding innovation clusters through collaborative research networks. Technovation 15 (9), 553-567. 
Lorence, D.P., Churchill, R., 2005. Incremental adoption of information security in health-care organizations: implications for document management. IEEE Trans. Inf. Technol. Biomed. 9, 169-73.

Lorenzi, N.M., Kouroubali, A., Detmer, D.E., Bloomrosen, M., 2009. How to successfully select and implement electronic health records (EHR) in small ambulatory practice settings. BMC Med. Inform. Decis. Mak. 9, 1-13. doi:10.1186/1472-6947-9-15

Ludwick D.A., Doucette, J., 2009. Adopting electronic medical records in primary care: lessons learned from health information systems implementation experience in seven countries. Int. J. Med. Inform. 78, 22-31. doi:10.1016/j.jjmedinf.2008.06.005

Lustria, M.L.A., Smith, S.A., Hinnant, C.C., 2011. Exploring digital divides: an examination of eHealth technology use in health information seeking, communication and personal health information management in the USA. Health Informatics J. 17, 224-43. doi:10.1177/1460458211414843

Mäenpää, T., SuoMäenpää, T., Suominen, T., Asikainen, P., Maass, M., \& Rostila, I. (2009). The outcomes of regional healthcare information systems in health care: a review of the research literature. International Journal of Medical Informatics, 78(11), 757-71. doi:10, T., Asikainen, P., Maass, M., Rostila, I., 2009. The outcomes of regional healthcare information systems in 
health care: a review of the research literature. Int. J. Med. Inform. 78, 75771. doi:10.1016/j.jimedinf.2009.07.001

Mahmood, A.M., Burn, J.M., Gemoets, L. A., Jacquez, C., 2000. Variables affecting information technology end-user satisfaction: a meta-analysis of the empirical literature. Int. J. Hum. Comput. Stud. 52, 751-771. doi:10.1006/ijhc.1999.0353

Makam, A.N., Lanham, H.J., Batchelor, K., Moran, B., Howell-Stampley, T., Kirk, L., Cherukuri, M., Samal, L., Santini, N., Leykum, L.K., Halm, E.A., 2014. The good, the bad and the early adopters: providers' attitudes about a common, commercial EHR. J. Eval. Clin. Pract. 20, 36-42. doi:10.1111/jep.12076

Malhotra, Y., 1999. Bringing the adopter back into the adoption process: A personal construction framework of information technology adoption. J. High Technol. Manag. Res. 10, 79-104. doi:10.1016/S1047-8310(99)80004-2

Malliou, P., Gioftsidou, A., Beneka, A., Godolias, G., 2006. Measurements and evaluations in low back pain patients. Scand. J. Med. Sci. Sports 16, 219 30. doi:10.1111/j.1600-0838.2005.00504.x

MarketsandMarkets, 2012. Next Generation Biometric Technologies Market Global Forecast \& Analysis (2012-2017). 
Marsh, K., Lanitis, T., Neasham, D., Orfanos, P., Caro, J., 2014. Assessing the value of healthcare interventions using multi-criteria decision analysis: A review of the literature. Pharmacoeconomics 32, 345-365. doi:10.1007/s40273-014-0135-0

Martich, G., Cervenak, J., 2007. Eyes wide shut. The "hidden" costs of deploying health information technology. J. Crit. Care 7-8.

Matheny, M.E., Ohno-Machado, L., 2007. Generation of Knowledge for Clinical Decision Support: Statistical and Machine Learning Techniques, in: Clinical Decision Support. pp. 227-248.

Matsueda, R.L., 2012. Key Advances in the History of Structural Equation Modeling, in: Hoyle, R.H. (Ed.), Handbook of Structural Equation Modeling. The Guilford Press, New York, pp. 17-42.

Matysiewicz, J., Smyczek, S., 2009. Consumer Trust - Challenge for Ehealthcare. 2009 Fourth Int. Conf. Coop. Promot. Inf. Resour. Sci. Technol. 333-338. doi:10.1109/COINFO.2009.40

Mayagoitia, R.E., Nene, A. V., Veltink, P.H., 2002. Accelerometer and rate gyroscope measurement of kinematics: An inexpensive alternative to optical motion analysis systems. J. Biomech. 35, 537-542. doi:10.1016/S00219290(01)00231-7 
McFarland, D.J., Hamilton, D., 2006. Adding contextual specificity to the technology acceptance model. Comput. Human Behav. 22, 427-447. doi:10.1016/j.chb.2004.09.009

McGinn, C.A., Grenier, S., Duplantie, J., Shaw, N., Sicotte, C., Mathieu, L., Leduc, Y., Légaré, F., Gagnon, M.-P., 2011. Comparison of user groups' perspectives of barriers and facilitators to implementing electronic health records: a systematic review. BMC Med. 9, 1-10. doi:10.1186/1741-7015-946

Meade, N., Islam, T., 2006. Modelling and forecasting the diffusion of innovation - A 25-year review. Int. J. Forecast. 22, 519-545.

doi:10.1016/j.ijforecast.2006.01.005

Meesapawong, P., Rezgui, Y., Li, H., 2014. Planning innovation orientation in public research and development organizations: Using a combined Delphi and Analytic Hierarchy Process approach. Technol. Forecast. Soc. Change 87, 245-256. doi:10.1016/j.techfore.2013.12.023

Mehta, D.D., Zañartu, M., Feng, S.W., Cheyne, H. a I., Hillman, R.E., 2012. Mobile voice health monitoring using a wearable accelerometer sensor and a smartphone platform. IEEE Trans. Biomed. Eng. 59, 3090-3096. doi:10.1109/TBME.2012.2207896 
Melas, C.D., Zampetakis, L. A., Dimopoulou, A., Moustakis, V., 2011. Modeling the acceptance of clinical information systems among hospital medical staff: an extended TAM model. J. Biomed. Inform. 44, 553-64. doi:10.1016/j.jbi.2011.01.009

Melles, R.B., Cooper, T., Peredy, G., 1998. User interface preferences in a pointof-care data system. Proc. AMIA Symp. 86-90.

Melone, N., 1990. A theoretical assessment of the user-satisfaction construct in information systems research. Manage. Sci. 36, 76-91.

Menachemi, N., Brooks, R.G., 2006. Reviewing the Benefits and Costs of Electronic Health Records and Associated Patient Safety Technologies. J. Med. Syst. 30, 159-168. doi:10.1007/s10916-005-7988-x

Menachemi, N., Matthews, M., Ford, E.W., Hikmet, N., Brooks, R.G., 2008. The Relationship Between Local Hospital IT Capabilities and Physician EMR Adoption. J. Med. Syst. 33, 329-335. doi:10.1007/s10916-008-9194-0

Mendonça, E. A., Chen, E.S., Stetson, P.D., McKnight, L.K., Lei, J., Cimino, J.J., 2004. Approach to mobile information and communication for health care. Int. J. Med. Inform. 73, 631-8. doi:10.1016/j.ijmedinf.2004.04.013 
Merkhofer, M.W., 1982. A process for technology assessment based on decision analysis. Technol. Forecast. Soc. Change 22, 237-265. doi:10.1016/00401625(82)90067-1

Metz, C.E., 1978. Basic principles of ROC analysis. Semin. Nucl. Med. 8, 283298. doi:http://dx.doi.org/10.1016/S0001-2998(78)80014-2

Miller, K.D., Waller, H.G., 2003. Scenarios, Real Options and Integrated Risk Management. Long Range Plann. 36, 93-107. doi:10.1016/S00246301(02)00205-4

Miller, R.H., Sim, I., 2004. Physicians' use of electronic medical records: barriers and solutions. Health Aff. (Millwood). 23, 116-26.

Möller, A., 2015. Leveraging Mobile Interaction with Multimodal and SensorDriven User Interfaces.

Möller, K., Kretschmer, J., Schranz, C., 2011. Hierarchical Modeling for Medical Decision Support, in: 2011 4th International Conference on Biomedical Engineering and Informatics (BMEI). pp. 960-964.

Moores, T.T., 2012. Towards an integrated model of IT acceptance in healthcare. Decis. Support Syst. 53, 507-516. doi:10.1016/j.dss.2012.04.014

Mordor Intelligence 2017. Wearable Medical Devices Market - Growth, Trends and Forecasts (2017-2022). 
Morton, M.E., Wiedenbeck, S., 2010. EHR acceptance factors in ambulatory care: a survey of physician perceptions. Perspect. Health Inf. Manag. 7, 1c.

Morton, M.E., Wiedenbeck, S., 2009. A framework for predicting EHR adoption attitudes: a physician survey. Perspect. Health Inf. Manag. 6, 1 a.

Mueller, R.O., Hancock, G.R., 2008. Best Practices in Structural Modeling, in: Best Practices in Quantitative Methods. SAGE Publications, Inc., 2455 Teller Road, Thousand Oaks California 91320 United States of America, p. 608. doi:10.4135/9781412995627

Mühlbacher, A.C., Kaczynski, A., 2016. Making Good Decisions in Healthcare with Multi-Criteria Decision Analysis: The Use, Current Research and Future Development of MCDA. Appl. Health Econ. Health Policy 14, 29-40. doi:10.1007/s40258-015-0203-4

Munos, B., Baker, P.C., Bot, B.M., Crouthamel, M., de Vries, G., Ferguson, I., Hixson, J.D., Malek, L.A., Mastrototaro, J.J., Misra, V., Ozcan, A., Sacks, L., Wang, P., 2016. Mobile health: the power of wearables, sensors, and apps to transform clinical trials. Ann. N. Y. Acad. Sci. 1375, 3-18. doi:10.1111/nyas. 13117

Murff, H.J., Gandhi, T.K., Karson, A.K., Mort, E.A., Poon, E.G., Wang, S.J., Fairchild, D.G., Bates, D.W., 2003. Primary care physician attitudes concerning follow-up of abnormal test results and ambulatory decision 
support systems. Int. J. Med. Inform. 71, 137-149. doi:10.1016/S13865056(03)00133-3

Nagy, P., George, I., Bernstein, W., Caban, J., Klein, R., Mezrich, R., Park, A., 2006. Radio frequency identification systems technology in the surgical setting. Surg Innov 13, 61-67. doi:10.1177/155335060601300110

Neal, T., Woodard, D., 2016. Surveying biometric authentication for mobile device security. J. Pattern Recognit. Res. 11, 74-110. doi:10.13176/11.764

Newman, J., 2009. OHSU Spine Center Closely Tracks Patient Outcomes To Determine Best Treatments [WWW Document]. URL http://www.ohsu.edu/xd/about/news_events/news/2009/ohsuspinecentertrac ksoutcomes.cfm (accessed 5.14.14).

Niskanen, R.O., 2002. The Oswestry Low Back Pain Disability Questionnaire. a two-year follow-up of spine surgery patients. Scand. J. Surg. 91, 208-11.

Noblin, A., Cortelyou-Ward, K., Cantiello, J., Breyer, T., Oliveira, L., Dangiolo, M., Cannarozzi, M., Yeung, T., Berman, S., 2013. EHR Implementation in a New Clinic: A Case Study of Clinician Perceptions. J. Med. Syst. 37, 9955. doi:10.1007/s10916-013-9955-2 
Ogunduyile, O.O., Zuva, K., Randle, O.A., Zuva, T., 2013. Ubiquitous Healthcare Monitoring System Using Integrated Triaxial Accelerometer, Spo2 and Location Sensors. Int. J. UbiComp 4, 1-13. doi:10.5121/iju.2013.4201

OHSU, 2015. OHSU Spine Center., pp.1-12.

Oortwijn, W., Banta, D., Vondeling, H., Bouter, L., 1999. Identification and priority setting for health technology assessment in The Netherlands: actors and activities. Health Policy (New. York). 47, 241-253.

Ortega Egea, J.M., Román González, M.V., 2011. Explaining physicians' acceptance of EHCR systems: An extension of TAM with trust and risk factors. Comput. Human Behav. 27, 319-332.

doi:10.1016/j.chb.2010.08.010

Overhage, J.M., 1996. Computer reminders to implement preventive care guidelines for hospitalized patients. Arch. Intern. Med. 156, 1551.

Pagemill Partners, 2014. Next-Generation User Interface Technologies for Mobile and Consumer Devices Next-Generation User Interface Technologies For Mobile / Consumer Devices. Extern. Rep.

Pai, F.-Y., Huang, K.-I., 2011. Applying the Technology Acceptance Model to the introduction of healthcare information systems. Technol. Forecast. Soc. Change 78, 650-660. doi:10.1016/j.techfore.2010.11.007 
Palacio, C., Harrison, J.P., Garets, D., 2009. Benchmarking Electronic Medical Records Initiatives in the US: a Conceptual Model. J. Med. Syst. 34, 273279. doi:10.1007/s10916-008-9238-5

Pallottino, S., Sechi, G., Zuddas, P., 2005. A DSS for water resources management under uncertainty by scenario analysis. Environ. Model. Softw. 20, 1031-1042. doi:10.1016/j.envsoft.2004.09.012

Pandejpong, T., Kocaoglu, D.F., 2002. Strategic Decision: Process for Technology Selection in the Petrochemical Industry. PICMET Proc.

Pantelopoulos, A., Bourbakis, N.G., 2010. A survey on wearable sensor-based systems for health monitoring and prognosis. IEEE Trans. Syst. Man, Cybern. Part C (Applications Rev. 40, 1-12.

doi:10.1109/TSMCC.2009.2032660

Paré, G., Sicotte, C., 2001. Information technology sophistication in health care: an instrument validation study among Canadian hospitals. Int. J. Med. Inform. 63, 205-23.

Park, H.-A., 2011. Pervasive Healthcare Computing. EMP/EHR, Wireless and Health Monitoring. Healthc. Inform. Res. 17, 89-91.

doi:10.4258/hir.2011.17.1.89 
Patel, S., Park, H., Bonato, P., Chan, L., Rodgers, M., 2012. A review of wearable sensors and systems with application in rehabilitation. J. Neuroeng. Rehabil. 9, 21. doi:10.1186/1743-0003-9-21

Pauker, S.G., 1976. Coronary artery surgery: the use of decision analysis. Ann. Intern. Med. 85, 8-18.

Pauker, S.G., Kassirer, J.P., 1987. Decision analysis. N. Engl. J. Med. 316, $250-$ 258.

Pearl, J., 1988. Probabilistic Reasoning in Intelligent Systems. MorganKaufmann, San Mateo, CA.

Phan, K., 2013. Innovation Measurement: A Decision Framework to Determine Innovativeness of a Company, Ph.D. thesis, Portland State University.

Phan, K., Kocaoglu, D.F., 2014. Innovation Measurement Framework to Determine Innovativeness of a Company : Case of Semiconductor Industry, in: 2014 Proceedings of PICMET'14: Infrustructure and Service Integration. pp. $747-757$.

Philips, 2013. The New Healthcare Enterprise. pp. 1-7.

Pilgrim, R., Hilton, J.A., Carrier, E., Pines, J.M., Hufstetler, G., Thorby, S., Milling, T.J., Cesta, B., Hsia, R.Y., 2010. Research priorities for administrative 
challenges of integrated networks of care. Acad. Emerg. Med. 17, 1330-6. doi:10.1111/j.1553-2712.2010.00934.x

Piliouras, T., Yu, P.L. (Raymond), Huang, H., Liu, X., Siddaramaiah, V.K.A., Sultana, N., 2011. Selection of electronic health records software: Challenges, considerations, and recommendations, in: 2011 IEEE Long Island Systems, Applications and Technology Conference. IEEE, pp. 1-5. doi:10.1109/LISAT.2011.5784239

Polančič, G., Heričko, M., Rozman, I., 2010. An empirical examination of application frameworks success based on technology acceptance model. J. Syst. Softw. 83, 574-584. doi:10.1016/j.jss.2009.10.036

Police, R.L., Foster, T., Wong, K.S., 2011. Adoption and use of health information technology in physician practice organisations : systematic review. Inform. Prim. Care 18, 245-259.

Poon, C., Zhang, Y.T., 2008. Perspectives on high technologies for low-cost healthcare: The Chinese scenario. IEEE Eng. Med. Biol. Mag. 27, 42-47. doi:10.1109/MEMB.2008.923955

Premier, 2015. Back injury prevention: safe patient handling [WWW Document]. URL http://www.premiersafetyinstitute.org/safety-topics-az/back-injuryprevention/extent-of-the-problem/ 
Premkumar, G., Bhattacherjee, A., 2008. Explaining information technology usage: A test of competing models. Omega 36, 64-75. doi:10.1016/j.omega.2005.12.002

Pynoo, B., Devolder, P., Duyck, W., Braak, J. Van, Sijnave, B., Duyck, P., 2011. Do hospital physicians' attitudes change during PACS implementation? A cross-sectional acceptance study. Int. J. Med. Inform. 81, 88-97. doi:10.1016/j.jjmedinf.2011.10.007

Ra, J.W., 1988. Analysis of expert judgments in hierarchical decision process, Ph.D. thesis, University of Pittsburgh.

Rahimpour, M., Lovell, N.H., Celler, B.G., McCormick, J., 2008. Patients' perceptions of a home telecare system. Int. J. Med. Inform. 77, 486-98. doi:10.1016/j.jjmedinf.2007.10.006

Raiffa, H., 1997. Decision analysis : introductory lectures on choices under uncertainty. McGraw-Hill, New York.

Randeree, E., 2007. Exploring Physician Adoption of EMRs: A Multi-Case Analysis. J. Med. Syst. 31, 489-496. doi:10.1007/s10916-007-9089-5

Redmond, S.J., Lovell, N.H., Yang, G.Z., Horsch, A., Lukowicz, P., Murrugarra, L., Marschollek, M., others, 2014. What Does Big Data Mean for Wearable Sensor Systems? Yearb Med Inf. 9, 135-142. doi:10.15265/IY-2014-0019 
Riddell, S., Wallace, W. A., 2011. The use of fuzzy logic and expert judgment in the R\&D project portfolio selection process. Int. J. Technol. Manag. 53, 238. doi:10.1504/IJTM.2011.038592

Rifat, L., 2013. Surgical Decision-Making Proces: More Questions than Answers. Scand. J. Surg. 102, 139-140.

Rind, D.M., Safran, C., 1993. Real and imagined barriers to an electronic medical record. Comput. Appl. Med. Care 74-78.

Robert Wood Johnson Foundation, 2010. Health Information Technology in the United States: Moving Toward Meaningful Use, 2010. pp.1-92.

Rodgers, M.M., Pai, V.M., Conroy, R.S., 2015. Recent Advances in Wearable Sensors for Health Monitoring. IEEE Sens. J. 15, 3119-3126. doi:10.1109/JSEN.2014.2357257

Rose, A.F., Schnipper, J.L., Park, E.R., Poon, E.G., Li, Q., Middleton, B., 2005. Using qualitative studies to improve the usability of an EMR. J. Biomed. Inform. 38, 51-60. doi:10.1016/j.jbi.2004.11.006

Rosemann, T., Marty, F., Bhend, H., Wagner, J., Brunner, L., Zoller, M., 2010. Utilisation of information technologies in ambulatory care in Switzerland. Swiss Med. Wkly. 140, w13088. doi:10.4414/smw.2010.13088 
Roth, C.P., Lim, Y.-W., Pevnick, J.M., Asch, S.M., McGlynn, E.A., 2009. The challenge of measuring quality of care from the electronic health record. Am. J. Med. Qual. 24, 385-94. doi:10.1177/1062860609336627

Russo, R.D.F.S.M., Camanho, R., 2015. Criteria in AHP: A systematic review of literature. Procedia Comput. Sci. 55, 1123-1132. doi:10.1016/j.procs.2015.07.081

Saaty, T.L., 1977. A Scaling Method for Priorities in Hierarchical Structures. J. Math. Psychol. 15, 234-281.

Sandberg, W.S., Ganous, T.J., Steiner, C., 2003. Setting a research agenda for perioperative systems design. Semin. Laparosc. Surg. 10, 57-70. doi:10.1177/107155170301000202

Sarasohn-Kahn, J., 2013. Making Sense of Sensors: How New Technologies Can Change Patient Care, California Healthcare Foundation.

Sayek, I., 2013. Surgery, surgeon, and measurement of value and quality of surgeons' work. Scand. J. Surg. 102, 141-4. doi:10.1177/1457496913489498

Schauberger, C.W., Larson, P., 2006. Implementing Patient Safety Practices in Small Ambulatory Care Settings. J. Qual. Patient Saf. 32, 419-425. 
Scheirey, S., Labs, H., Soubra, D., 2013. Sensor Fusion, Sensor Hubs and the Future of Smartphone Intelligence.

Schepers, J., Wetzels, M., 2007. A meta-analysis of the technology acceptance model: Investigating subjective norm and moderation effects. Inf. Manag. 44, 90-103. doi:10.1016/j.im.2006.10.007

Schoen, C., Osborn, R., Huynh, P.T., Doty, M., Peugh, J., Zapert, K., 2006. On the front lines of care: primary care doctors' office systems, experiences, and views in seven countries. Health Aff. (Millwood). 25, w555-71. doi:10.1377/hlthaff.25.w555

Schwartz, L., Miller, R., Plummer, D., Fusfeld, A.R., 2011. Measuring the Effectiveness of R\&D. Res. Manag. 54, 29-36. doi:10.5437/08956308X5405008

Schwartz, W.B., Gorry, G.A., Kassirer, J.P., Essig, A., 1973. Decision analysis and clinical judgment. Am. J. Med. 55, 459-472.

Scott, P.J., Briggs, J.S., 2009. A Pragmatist Argument for Mixed Methodology in Medical Informatics. J. Mix. Methods Res. 3, 223-241. doi:10.1177/1558689809334209 
Seneler, C.O., Basoglu, N., Daim, T., 2009. Interface feature prioritization for web services: Case of online flight reservations. Comput. Human Behav. 25, 862-877. doi:10.1016/j.chb.2008.12.028

Shany, T., Redmond, S.J., Narayanan, M.R., Lovell, N.H., 2012. Sensors-based wearable systems for monitoring of human movement and falls. IEEE Sens. J. 12, 658-670. doi:10.1109/JSEN.2011.2146246

Sharif, M.N., Sundararajan, V., 1983. A quantitative model for the evaluation of technological alternatives. Technol. Forecast. Soc. Change 24, 15-29. doi:10.1016/0040-1625(83)90060-4

Sheikh, N.J., Park, Y., Kocaoglu, D.F., 2014. Assessment of Solar Photovoltaic Technologies Using Multiple Perspectives and Hierarchical Decision Modeling : Manufacturers Worldview, in: 2014 Proceedings of PICMET'14: Infrastructure and Service Integration. pp. 491-497.

Sheikh, N.J., 2013. Assessment of Solar Photovoltaic Technologies Using Multiple Perspectives and Hierarchical Decision Modeling, Ph.D. thesis, Portland State University.

Shen, J.J., Ginn, G.O., 2012. Financial position and adoption of electronic health records: a retrospective longitudinal study. J. Health Care Finance 38, 6177. 
Shen, Y.-C., Chang, S.-H., Lin, G.T.R., Yu, H.-C., 2010. A hybrid selection model for emerging technology. Technol. Forecast. Soc. Change 77, 151-166. doi:10.1016/j.techfore.2009.05.001

Shields, A.E., Shin, P., Leu, M.G., Levy, D.E., Betancourt, R.M., Hawkins, D., Proser, M., 2007. Adoption of health information technology in community health centers: results of a national survey. Health Aff. (Millwood). 26, 137383. doi:10.1377/hlthaff.26.5.1373

Shin, D.-H., 2010. The effects of trust, security and privacy in social networking: A security-based approach to understand the pattern of adoption. Interact. Comput. 22, 428-438. doi:10.1016/j.intcom.2010.05.001

Simon, S., Kaushal, R., Cleary, P., Jenter, C., 2007. Correlates of electronic health record adoption in office practices: a statewide survey. J. Am. Med. Informatiics Assoc. 14, 110-117. doi:10.1197/jamia.M2187.Introduction

Simon, S., McCarthy, M., Kaushal, R., Jenter, C., Volk, L., Poon, E., Yee, K.C., Orav, J., Williams, D., Bates, D., 2008. Electronic health records: which practices have them, and how are clinicians using them? J. Eval. Clin. Pract. $14,43-47$.

Slade Shantz, J.A., Veillette, C.J.H., 2014. The application of wearable technology in surgery: ensuring the positive impact of the wearable 
revolution on surgical patients. Front. Surg. 1, 39.

doi:10.3389/fsurg.2014.00039

Soh, P.J., Vandenbosch, G.A.E., Mercuri, M., Schreurs, D.M.M.-P., 2015.

Wearable wireless health monitoring. IEEE Microw. Mag. 55-70.

Soreon Research, 2014. The Wearable Health Revolution: How Smart Wearables Disrupt the Healthcare Sector 1-27.

Startup Health, 2015. StartUp Health Insights: Digital Health Funding Rankings 2015 Midyear Report 1-22.

Stavropoulos, T.G., Meditskos, G., Andreadis, S., Kompatsiaris, I., 2015. Realtime health monitoring and contextualised alerts using wearables. Proc. 2015 Int. Conf. Interact. Mob. Commun. Technol. Learn. IMCL 2015 358363. doi:10.1109/IMCTL.2015.7359619

Stoaks, U., 2014. Re-imagining Health and Wellness. StartUp Health Insights Q1 2014 [WWW Document]. URL http://www.slideshare.net/StartUpHealth/startup-health-insights-q1-2014-update (accessed 5.14.14).

Storey, J., Buchanan, D., 2008. Healthcare governance and organizational barriers to learning from mistakes. J. Heal. Organ. Manag. 22, 642-651. doi:10.1108/14777260810916605 
Strome, T.L., 2013. Healthcare Analytics for Quality and Performance Improvement. Wiley, Hoboken.

Swets, J.A., 1979. ROC analysis applied to the evaluation of medical imaging techniques. Invest. Radiol. 14, 109-121.

Szajna, B., 1996. Empirical evaluation of the revised technology acceptance model. Manage. Sci. 42, 85-92.

Tanoglu, I., Basoglu, N., Daim, T., 2010. Exploring Technology Diffusion: Case of Information Technologies. Int. J. Inf. Technol. Decis. Mak. 9, 195-222. doi:10.1142/S0219622010003786

Teng, X.F., Zhang, Y.T., Poon, C.C.Y., Bonato, P., 2008. Wearable medical systems for p-Health. IEEE Rev. Biomed. Eng. 1, 62-74. doi:10.1109/RBME.2008.2008248

Thokala, P., Duenas, A., 2012. Multiple criteria decision analysis for health technology assessment. Value Health 15, 1172-81. doi:10.1016/j.jval.2012.06.015

Thorn, V., Hunt, F., Mitchell, R., Probert, D., Phaal, R., 2011. Internal technology valuation: real world issues. Int. J. Technol. Manag. 53, 149-160. 
Tobergte, D.R., Curtis, S., 2013. MEMS accelerometers target healthcare applications. J. Chem. Inf. Model. 53, 1689-1699.

doi:10.1017/CBO9781107415324.004

Tran, T. A., Daim, T., 2008. A taxonomic review of methods and tools applied in technology assessment. Technol. Forecast. Soc. Change 75, 1396-1405. doi:10.1016/j.techfore.2008.04.004

Tsiknakis, M., Katehakis, D.G., Orphanoudakis, S.C., 2002. An open, component-based information infrastructure for integrated health information networks. Int. J. Med. Inform. 68, 3-26.

Turan, T., Amer, M., Tibbot, P., Almasri, M., Fayez, F. Al, Graham, S., 2009. Use of Hierarchal Decision Modeling ( HDM ) for selection of Graduate School for Master of Science Degree Program in Engineering. PICMET 2009 Proc. $535-549$.

Tyler, J.L., 2001. The healthcare information technology context: a framework for viewing legal aspects of telemedicine and teleradiology. Proc. 34th Annu. Hawaii Int. Conf. Syst. Sci. 10. doi:10.1109/HICSS.2001.926562

Valdes, I., Kibbe, D., Tolleson, G., Kunik, M., Peterson, L., 2004. Barriers to proliferation of electronic medical records. Inform. Prim. Care 12, 3-9. 
Van Blommestein, K.C., Daim, T.U., 2013. Residential energy efficient device adoption in South Africa. Sustain. Energy Technol. Assessments 1, 13-27. doi:10.1016/j.seta.2012.12.001

Van Genugten, M.L.L., Heijnen, M.-L.A., Jager, J.C., 2003. Pandemic Influenza and Healthcare Demand in the Netherlands: Scenario Analysis. Emerg. Infect. Dis. 9, 531-538.

Van Lummel, R.C., Ainsworth, E., Lindemann, U., Zijlstra, W., Chiari, L., Van Campen, P., Hausdorff, J.M., 2013. Automated approach for quantifying the repeated sit-to-stand using one body fixed sensor in young and older adults. Gait Posture 38, 153-156. doi:10.1016/j.gaitpost.2012.10.008

Van Schaik, P., Flynn, D., Van Wersch, A., Douglass, A., Cann, P., 2004. The acceptance of a computerised decision-support system in primary care: A preliminary investigation. Behav. Inf. Technol. 23, 321-326. doi:10.1080/0144929041000669941

Vedvik, E., Tjora, A.H., Faxvaag, A., 2009. Beyond the EPR: complementary roles of the hospital-wide electronic health record and clinical departmental systems. BMC Med. Inform. Decis. Mak. 9, 29. doi:10.1186/1472-6947-9-29

Venkatesh, V., Davis, F., 2000. A theoretical extension of the technology acceptance model: Four longitudinal field studies. Manage. Sci. 46, 186204. 
Vishwanath, A., Scamurra, S.D., 2007. Barriers to the adoption of electronic health records: Using concept mapping to develop a comprehensive empirical model. Health Informatics J. 13, 119-134.

Vishwanath, A., Brodsky, L., Shaha, S., 2009. Physician adoption of personal digital assistants (PDA): testing its determinants within a structural equation model. J. Health Commun. 14, 77-95. doi:10.1080/10810730802592270

Viswanathan, S., 2005. Competing across technology-differentiated channels: The impact of network externalities and switching costs. Manage. Sci. 51, 483-496.

VitalConnect, 2017. VitalPatch [WWW Document]. URL https://vitalconnect.com/solutions/vitalpatch/

Vondeling, H., Rosendal, H., Banta, D., 1995. Evaluation and diffusion of excimer laser treatment of myopia in the United States and in the Netherlands. Health Policy (New. York). 34, 21-33.

Wagner, H., Weibel, S., 2005. The Dublin Core Metadata Registry: Requirements, Implementation, and Experience. JoDI J. Digit. Inf. 1-20.

Walker, J., Carayon, P., Leveson, N., Paulus, R., 2008. EHR Safety: The Way Forward to Safe and Effective Systems. J. Am. Med. Informatics Assoc. 15, 272-277. doi:10.1197/jamia.M2618.Introduction 
Walter, F., 2008. Process-Orientation and Core Competence Management the Contribution of the Process-Oriented Core Competence Management to the Integration of the Market-based View and the Resource-based View, Dr. rer. pol. thesis, Technischen Universität Chemnitz.

Wang, B., Kocaoglu, D.F., Daim, T., Yang, J., 2010. A Decision Model for Energy Resource Selection in China. Energy Policy 38, 7130-7141.

Wang, B., Hsieh, C.-H., 2014. Measuring the value of patents with fuzzy multiple criteria decision making: insight into the practices of the Industrial Technology Research Institute. Technol. Forecast. Soc. Change. doi:10.1016/j.techfore.2014.09.015

Wang, B., Narvekar, K., Nguyen, T., 2013. The Hierarchical Decision Model for Non-Profit Organization's Project Selection, in: 2013 Proceedings of PICMET'13: Technology Management for Emerging Technologies. pp. 401410.

Wannenburg, J., Malekian, R., 2015. Body Sensor Network for Mobile Health Monitoring, a Diagnosis and Anticipating System. IEEE Sens. J. 15, 68396852. doi:10.1109/JSEN.2015.2464773

Warren, S., Lebak, J., Yao, J., Creekmore, J., Milenkovic, A., Jovanov, E., 2005. Interoperability and security in wireless body area network infrastructures. 
Conf. Proc. ... Annu. Int. Conf. IEEE Eng. Med. Biol. Soc. IEEE Eng. Med. Biol. Soc. Annu. Conf. 4, 3837.

Watson, R.H., 1978. Interpretive structural modeling—A useful tool for technology assessment? Technol. Forecast. Soc. Change 11, 165-185. doi:10.1016/0040-1625(78)90028-8

Wearable Technology, 2014. Metria IH1: The Evolution In Wearable Tech! [WWW Document]. URL http://www.wearabletechdigest.com/metria-ih1-theevolution-in-wearable-tech.html

Weiner, B.J., Amick, H.R., Lund, J.L., Lee, S.-Y.D., Hoff, T.J., 2011. Use of qualitative methods in published health services and management research: a 10-year review. Med. Care Res. Rev. 68, 3-33. doi:10.1177/1077558710372810

Were, M.C., Emenyonu, N., Achieng, M., Shen, C., Ssali, J., Masaba, J.P.M., Tierney, W.M., 2010. Evaluating a scalable model for implementing electronic health records in resource-limited settings. J. Am. Med. Inform. Assoc. 17, 237-44. doi:10.1136/jamia.2009.002303

Wilhite, A.W., 2004. Estimating the Risk of Technology Development., pp.14-30. 
Wilson, E., Lankton, N., 2004. Modeling patients' acceptance of providerdelivered e-health. J. Am. Med. Informatics Association. 11, 241-248. doi:10.1197/jamia.1475.Health-related

Witter, D.M., 2009. Oregon Electronic Health Record Survey Report: Ambulatory Practices and Clinics, Health Policy., pp. 1-155.

Wolf, J.A., Niederhauser, V., Marshburn, D., Lavela, S.L., 2014. Defining patient experience. Patient Exp. J. 1, 7-19.

Wong, D.H., 2003. Changes in intensive care unit nurse task activity after installation of a third-generation intensive care unit information system. Crit. Care Med. 31, 2488.

World Health Organization, 2008. Integrated health services-what and why? Tech. Br. No. 11-10.

Wu, J.-H., Chen, Y.-C., Greenes, R.A., 2009. Healthcare technology management competency and its impacts on IT-healthcare partnerships development. Int. J. Med. Inform. 78, 71-82. doi:10.1016/j.jjmedinf.2008.05.007

Wu, J.-H., Wang, S.-C., Lin, L.-M., 2007. Mobile computing acceptance factors in the healthcare industry: a structural equation model. Int. J. Med. Inform. 76, 66-77. doi:10.1016/j.ijmedinf.2006.06.006 
Yamamoto, K., Matsumoto, S., Tada, H., Yanagihara, K., Teramukai, S., Takemura, T., Fukushima, M., 2008. A Data Capture System for Outcomes Studies that Integrates with Electronic Health Records: Development and Potential Uses. J. Med. Syst. 32, 423-427. doi:10.1007/s10916-008-9147-7

Yang, H., 2004. It's all about attitude: revisiting the technology acceptance model. Decis. Support Syst. 38, 19-31.

Yao, W., Kumar, A., 2013. CONFlexFlow : Integrating Flexible clinical pathways into clinical decision support systems using context and rules. Decis. Support Syst. 55, 499-515. doi:10.1016/j.dss.2012.10.008

Yarbrough, A.K., Smith, T.B., 2007. Technology acceptance among physicians: a new take on TAM. Med. Care Res. Rev. 64, 650-72. doi:10.1177/1077558707305942

Yeager, V. A, Menachemi, N., Brooks, R.G., 2010. EHR adoption among doctors who treat the elderly. J. Eval. Clin. Pract. 16, 1103-7. doi:10.1111/j.13652753.2009.01277.x

Yi, M.Y., Jackson, J.D., Park, J.S., Probst, J.C., 2006. Understanding information technology acceptance by individual professionals: Toward an integrative view. Inf. Manag. 43, 350-363. doi:10.1016/j.im.2005.08.006 
Yoon-Flannery, K., Zandieh, S.O., Kuperman, G.J., Langsam, D.J., Hyman, D., Kaushal, R., 2008. A qualitative analysis of an electronic health record (EHR) implementation in an academic ambulatory setting. Inform. Prim. Care 16, 277-285.

Yu, P., Li, H., Gagnon, M.-P., 2009. Health IT acceptance factors in long-term care facilities: a cross-sectional survey. Int. J. Med. Inform. 78, 219-29. doi:10.1016/j.jjmedinf.2008.07.006

Yusof, M.M., Kuljis, J., Papazafeiropoulou, A., Stergioulas, L.K., 2008. An evaluation framework for Health Information Systems: human, organization and technology-fit factors (HOT-fit). Int. J. Med. Inform. 77, 386-98. doi:10.1016/j.ijmedinf.2007.08.011

Zandieh, S.O., Yoon-Flannery, K., Kuperman, G.J., Langsam, D.J., Hyman, D., Kaushal, R., 2008. Challenges to EHR implementation in electronic- versus paper-based office practices. J. Gen. Intern. Med. 23, 755-61. doi:10.1007/s11606-008-0573-5

Zaroukian, M.H., 2006. Benefiting from Ambulatory EHR Implementation: Solidarity, Six Sigma, and Willingness to Strive. JHIM 20, 53-60.

Zhang, R., Liu, L., 2010. Security models and requirements for healthcare application clouds. Proceedings of the 2010 IEEE. 3rd International Conference on Cloud Computing (pp. 268-275). Miami, FL: IEEE. 
Zhang, Q., Ishihara, K.N., Mclellan, B.C., Tezuka, T., 2012. Scenario analysis on future electricity supply and demand in Japan. Energy 38, 376-385. doi:10.1016/j.energy.2011.11.046

Zheng, K., Padman, R., Krackhardt, D., Johnson, M.P., Diamond, H.S., 2010. Social networks and physician adoption of electronic health records: insights from an empirical study. J. Am. Med. Inform. Assoc. 17, 328-36. doi:10.1136/jamia.2009.000877

Zhou, J., Cao, Z., Dong, X., Lin, X., 2015. Security and privacy in cloud-assisted wireless wearable communications: Challenges, solutions, and future directions. IEEE Wirel. Commun. 22, 136-144.

doi:10.1109/MWC.2015.7096296

Zhou, L., Karipineni, N., Lewis, J., Maviglia, S.M., Fairbanks, A., Hongsermeier, T., Middleton, B., Rocha, R.A., 2012. A study of diverse clinical decision support rule authoring environments and requirements for integration. BMC Med. Inform. Decis. Mak. 12, 128. doi:10.1186/1472-6947-12-128

Zhou, Y., Minshall, T., Hampden-Turner, C., 2010. Building Innovation Capabilities: an Inquiry Into the Dynamic Growth Process of University SpinOuts in China. Int. J. Innov. Technol. Manag. 7, 273-302.

doi:10.1142/S0219877010002082 
Zijlstra, W., Bisseling, R.W., Schlumbohm, S., Baldus, H., 2010. A body-fixedsensor-based analysis of power during sit-to-stand movements. Gait Posture 31, 272-278. doi:10.1016/j.gaitpost.2009.11.003 
APPENDICES 


\section{Appendix A - Research Invitation Letter}

\section{Assessment of Technology Adoption Potential of Medical Devices: Case of Wearable Sensor Products for Pervasive Care in Neurosurgery and Orthopedics}

My name is Liliya Hogaboam, and I am a Ph.D. Candidate at the Department of Engineering and Technology Management (ETM), at Portland State University. I am conducting a research study to develop a health information technology assessment framework for technology selection with a case of wearable sensor technologies in spine health.

You are being asked to take part in this research because you are considered an expert in the area of leadership and management in healthcare industry, policy implementation, system thinking and information technologies in healthcare due to your qualification and professional experience. As one of the leading experts in this area, I would like to get your expert judgement for the criteria in technology selection in spine health. The case study I am using is to evaluate wearable sensor technologies for improving a spine health patient care database in neurosurgery and orthopedics. Your participation will help increase the knowledge of health technology assessment and factors important for technology selection in a healthcare setting. This study is being conducted in partial fulfillment of the requirements for a doctoral degree in Technology Management at Portland State University.

If you decide to participate, an Informed Consent Form will be sent to you and require your signature before starting the research. I will be sending you some data collection instruments after I receive the signed form. You will be asked to provide your opinions towards the research criteria. The research instrument will take about 15 minutes to complete. Afterwards, I will quantify the judgmental data and prioritize the related research criteria.

There is no risk for the involvement in this research. No personally identifiable data is collected, and will not be reported. All data will remain confidential. The data obtained from the participants will only be reported in aggregate format, and individual information will be kept confidential. All responses will be concealed, and no one other than the researcher will have access to them. The information will be deleted or destroyed by the researcher within one year after the completion of this research.

The benefits for participation include two things: 1) at the end of the research, a copy of the aggregated results will be provided to you at no cost; 2) through your participation as an expert, researcher, and decision-maker, we will all learn more about which wearable sensor technology solutions are most important to engage in for improving spine patient care database to potentially have a positive impact on spine patient care and what criteria play the most important role in this technology selection. The model is generalizable and can ultimately be applied to benefit many other types of organizations. 
Participation in this research is completely voluntary. You have the right to withdraw at any time or refuse to participate entirely and it will not affect your relationship with the investigator or any institute.

If you have questions regarding this study, you may contact Liliya Hogaboam at ${ }^{* * * * * * *} @$ pdx.edu, ${ }^{* * * * *} @{ }^{* * * * * * * *}$.com or $\left(^{* * *}\right)^{* * *-}{ }^{* * * *}$. If you have concerns or problems about your participation in this study or your rights as a research subject, please contact the Human Subjects Research Review Committee, Research and Strategic Partnerships, Market Center Building, 6th Floor, 1600 SW 4th Ave, Portland, OR 97201, (503) 725-4288 / 1-877-480- 4400.

Sincerely, Liliya Hogaboam

Ph.D. Candidate

Department of Engineering and Technology Management

Portland State University

Follow up letter:

Dear Dr.

I contacted you several months ago to obtain your expert opinion in my research study. Thank you for agreeing to participate! Before quantification of my model, it needs to be validated and I would kindly ask you to answer yes and no statements next to the model criteria. Since you'll be evaluating select (not all) criteria, this should take just a few minutes. Again, thank you so much for your help.

Below is the link to the validation questions. The password for validation questionnaire:

https://portlandstate.qualtrics.com/.

Please let me know if you have any questions and if you have any issues with the link.

Sincerely,

Liliya Hogaboam, Ph.D. Candidate

Department of Engineering and Technology Management (ETM)

Portland State University

Current dissertation title: "Assessment of Technology Adoption Potential of Medical Devices: Case of Wearable Sensor Products for Pervasive Care in Neurosurgery and Orthopedics". 


\title{
Appendix B - Validation Questionnaire
}

\author{
Model validation
}

Dear Sir/Madam,

You are invited to participate in a research study conducted by Liliya Hogaboam from Portland State University, Engineering and Technology Mansgement Department. The study aims to research and assess technology adoption potential of medical devices using hierarchical decision modeling and multi-perspective approach. A case study of evaluating wearable sensor products for pervasive care in neurosurgery and orthopedics will be used to demonstrate the model's capability. This project is being conducted in partial fulfillment for the requirements of a PhD degree under the supervision of Dr. Tugrul U. Daim and Dr. Dundar F. Kocaoglu, You are invited as a potential participant due to your expertise in the area of spine health patient care due to your qualification and professional experience.

Before the model is quantified, the important step is to validate the researched criteria. If you decide to participate, you will be asked to provide your expert opinion to validate criteria of the model pertaining to your field of expertise. This is Yes/No validation process, thus it presents no hazard to the participants. The task takes about 5 to 10 minutes to complete. You will not receive any direct benefit from taking part in this study, but the study may help to increase knowledge which may help others in the future.

Your name and responses will be confidential and will not be shared with any third party. Any data linked to your identification will be stored in a secured place only accessible by the researcher. The data will be destroved within one year after the completion of the study, Participation in this research is totally voluntary and you have the right to withdraw at any time without affecting your relationship with the researcher of any institute.

If you have concerns or problems about your participation in this study or your nights as a research subject, please contact the Human Subjects Research Review Committee, Office of Research Strategic Partnerships, 1600 SW Fourth Avenue, Sulte 620, Portland, OR, 97201, (503) 7253423 . If you have any questions about the study itself, contact Liliya Hogaboam: hliiya@pdx.edu, liiyasenascentia. com or (503) $750-9129$

Your signature indicales that you have read and understand the above information and agree to take part in this study. Please understand that you may Withdraw your consent at any time without penaty, and that by signing, you are not waiving any lagal claims, nghts or remedies.

Signature (Type your name)

Date (mm/dd/yyyy) 


\section{Assessment of Technology Adoption Potential of Medical Devices: Case of Wearable Sensor Products for Pervasive Care in Neurosurgery and Orthopedics}

The research study aims to assess technology adoption potential of wearable sensor products in spine care using hiefarchical decision modeling and multi-perspective approach.

A case study of evaluating wearable sensor technology solutions for improving spine health patient care database in neurosurgery and orthopedics will be used to demonstrate the model's capability.

The objective of this research is assessment of technology adoption potential through multiple perspectives view by implementation of a hierarchical decision-making model (HDM).

The development of the model components known as criteria, subcriteria and alternatives has been done through a comprehensive literature review.

Validation tests whether the model is an adequate representation of the elements and their relationships of the actual systems it's referring to. In case you might have difficulty with the terms, please refer to the explanations for the terms listed.

Please refer to figure below for more information about the preliminary model: 


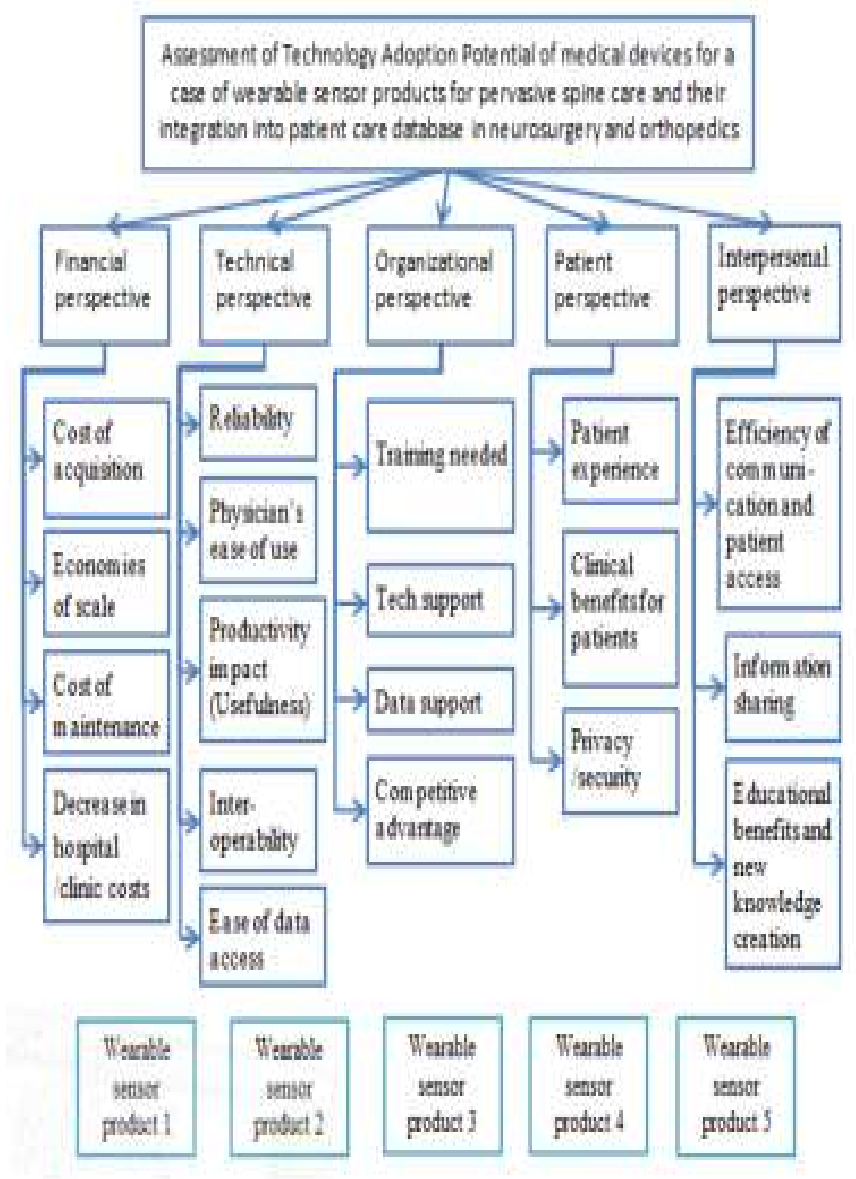

Literature review has revealed that there are five major perspectives associated with health technology adoption. Those perspectives have relative importance values for the decision-maker. Brief explanations of each of the perspectives are presented below:

Financial perspective is mentioned in the literature from rising costs of healthcare to high costs of systems implementation and management as well as loses from unsuccessul software applications, It encompasses the importance of financial benefits to the organization that a certain technology selection could bring.

Technical perspective represents the value of a technology in a healthcare setting from the view of the importance of technical capablitities improvement. 
Organizational perspective encompasses the criteria that technological solutions should provide in organizational setting, which includes management support, integration, tech and data support as well as comperitive advantage it can provide for the heathcare organization.

Patient perspective reflects criteria that reflect physician's personal issues that technological solution needs to provide like satisfactory patient experience, clinical benefits and privacy and security issues.

Interpersonal perspective reflects the importance of sharing and communication among various levels in the organization and outside; the ability of the technological solution to provide that capability and perhaps improve the communication and important flow of information.

The following criteria were selected based on extensive literature review in healthcare technology adoption and opinions of researchers and leaders in the field. Please validate the importance of the first level criteria towards the main goal of assessment of adoption potential for a case of wearable sensor products for spine health in neurosurgery and orthopedics. Vote "yes" if you think the criteria belongs in the model or "no" if it doesn't.

Yes No

Financial perspective

Technical perspective

Organizational perspective

Patient perspective

Interpersonal perspective

Other (Click to write)
0 o

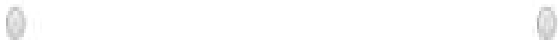

$\theta-6$

$0+0$

0

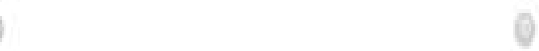

Cost of acquisition - Expenses associaled with the purchase of the wearable sensor technology solution

Economies of scale - Savings to the department and clinic due to obtaining large amounts of a particular kind of wearable technological soluton for their patients (prowded by the suppsilers and by the larget pool of paltents sevved by a wearable technological solution)

Cost of maintenance - Cosis assoctaled whih maintenance of the system of the wearabie technological soluliton

Decrease in hospitaliclinic costs - Decrease in overall expenses incurred by hospital in providing services due to the technology acquisition 
The following criteria were selected based on extensive literature review in healthcare technology adoption and opinions of researchers and leaders in the field, Please validate the importance of the second level criteria related to Financial perspective, Vote "yes" if you think the criteria belongs in the model or "no" if it doesn't.

Yes No

Cost of acquisition

Economies of scale

Cost of maintenance

Decrease in hospital/clinic

costs

Other (Click to write)
0

0

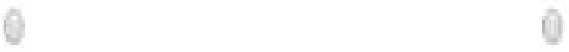

(

9

Reliability - A charactenstic of a wearable sonsor technobgical solviton prowding accuratie measurements and analytic reports. consistant and stable performance, free of tecthical ssues and prodems.

Physician's Ease of Use - Physican's intulfive and effortiess interaction with the wearable sensor technological solution incudingy the accompanied soltware, databases and analytics/aggregate roports

Productivity Impact (Usefulness) - The ability of the completo technological solution to enthance the physician's job performance (enabling to provice better care, diggnoses, atci)

Interoperability - Vendor's support of consensus standards that enables communication and datta exchange of wearable sannor technology solution with cilinical sothware appicastlons and dalabases.

Ease of Data Access - Ability of the wearable sensor solution to provide easy access to data reports and analytics.

The following criteria were selected based on extensive literature review in healthcare technology adoption and opinions of researchers and leaders in the field. Please valdate the importance of the second level criteria related to Technical perspective. Vote "yes" if you think the criteria belongs in the model or "no" if it doesn't.

Reliability

Physician's Ease of Use
Yes

No

(1)

0 
Productivity Impact

(Usefulness)

Interoperability

Ease of Data Access

Other (Click to write)

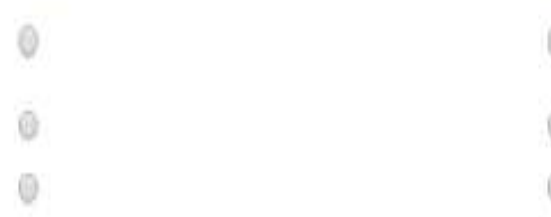

(ath to wite)

Training needed - Organized activity aimed at imparting information and /or instructions to help medical staff attain a required level of knowledge or skill related to wearable sensor technological solution

Tech Support. A service provided by a hardware or software company that provides medical staff with help and advice about the wearable sensor technology solution

Data Support - Support with overall data integration: formats, standards, changes, downloads, updates, changes esc.

Competitive advantage - An advantage over competiors gained by hospltals/dinics offering patients greater value by providing greater benefis and services as a results of the wearable sensor technology implementation

The following criteria were selected based on extensive literature review in healthcare technology assessment and opinions of researchers and leaders in the field. Please validate the importance of the second level criteria related to Organizational perspective. Vote "yes" if you think the criteria belongs in the model or "no" if it doesn't.

$\begin{array}{lcc} & \text { Yes } & \text { No } \\ \text { Training Needed } & 0 & 0 \\ \text { Tech support } & 0 & 0 \\ \text { Data support } & 0 \\ \text { Competitive advantage } & 0 \\ \text { Other (Click to write) } & 0\end{array}$


Patient Experience - Overal patien's satsfaction with using wearable solution

Clinical Benefits for Patients - Health outcome measures a positive effect of a therapeutic intervention (improvement in patient's mobiily, improvement in acherence to the treatment plan and behavioral intervention)

Privacy/Security, Protection of personal informaton

The following criteria were selected based on extensive iterature review in healthcare technology adoption and opinions of recearchers and leaders in the field. Please validate the importance of the second level criteria related to Patient perspective. Vote "yes" if you think the criteria belongs in the model or "no" if it doesn't.

Patient Experience

Clinical Benefits for Patients

Privacy/Security

Other (Click to write)
Yes No

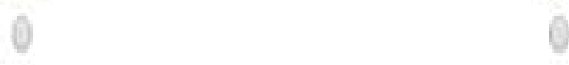

(2)

9

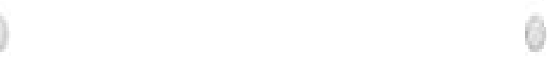

Efficiency of Communication and Patient Access - The ability of the wearable solution to improve interpersonal connectivity (among healthcare team members and the patient) and access to patlents as part of remote health care model

Information Sharing - The ability of new technological solutions to share information with varlous internal and external healthcare providers and shared decision-makers

Educational Benefits and New Knowledge Creation - Contributing to educational benefits and new knowledge creation for the parties involved

The following criteria were selected based on extensive lterature review in healthcare technology adoption and opinions of researchers and leaders in the field. Please validate the importance of the second level criteria related to Interpersonal perspective. Vote "yes" if you think the criteria belongs in the model or "no" if it doesn't. 
Efficiency of

Communication and Patient

Access

Information Sharing

Educational Benefits and

New Knowledge Creation

Other (Click to write)

\section{Wearable Sensor Products:}

Metria IH1 by Vancive Medical Technologies - a disposabie Itestyle assessmert system that oullects and reports multiple parameters tox health, fiterss and weliness purposes

- 3-axis accelerometer + skin temperature sensor, near body temperature and galvanic skin response (GSR)

VITALITI by Cloud DX - medically accurate consumer/cilinical vital sign plattorm

- 3-axis accelerometer and additional sensors for measuring vitality (electrocardiograph, photoplethysmograph, core thermometer, oscillometric cuff (BP), microphone (Coughs), spirometer module, wireless camera)

HealthPatchMD by VitalConnect - continuous remote patient monitoring and a comprehensive tracking of eight FDA-cleared measurements in a single biosensor patch

- 3-axis MEM5 accelerometer to detect motion, ECG electrodes to detect heart rate, thermistor to detect skin temperature

Valedo by Hocoma - back pain therapy medical device with constant feedback and motivation through tracking performance and progress sharing

- 3D gyroscope +30 accelerometer $+3 D$ magnetometer (a continuous and precise 9-axis vector calculation of movement angles and acceierations)

Opal by ADPM, Inc, - research-grade wearable sensor with robust software development kit built for measuring activity level, tremor, intervention response, gait, balance, diumal patterns and turning 
- 3-axis sccelerometer +3 -axis gyroscope +3 -axis magnetometer

The following criteria were selected based on extensive literature review in healthcare technology adoption and opinlons of researchers and leaders in the field. Please validate the importance Alternatives level. Vote "yes" if you think the selection belongs in the model of "no" if it doesn't,

Yes No

Metria IH1

VITALITI

HealthPatchMD

Valedo

Opal

(1)

0

0

0

(

Other (Click to write)

Powered by Qualtrics 


\title{
Appendix C - Quantification Instrument -Qualtrics
}

\author{
(full instrument is available from the author)
}

Dear Sir/Madam,

You are invited to participate in a research study conducted by Liliya Hogaboam, a Ph.D. Candidate at Portland State University, Engineering and Technology Management Department. The study aims to research and assess technology adoption potential of medical devices using hierarchical decision modeling and multi-perspective approach. A case study of evaluating wearable sensor products for pervasive care in neurosurgery and orthopedics will be used to demonstrate the model's capability. This project is being conducted in partial fulfillment for the requirements of a PhD degree under the supervision of Dr. Tugrul U. Daim. You have been selected as a potential participant for your expertise in the area of spine health patient care, leadership role in healthcare management, your qualifications and professional experience. You will be participating in an evaluation and quantification of selected groups of the model criteria.

You will be asked to provide your opinions by doing pairwise comparisons among the research criteria. The research instrument will take about 15-20 minutes to complete. When you return the research instruments, I will quantify the data and prioritize the related research criteria.

The benefits for participation include two things: 1) at the end of the research, a copy of the aggregated results will be provided to you at no cost; 2) through your participation as an expert, researcher, and decision-maker, we will broaden our understanding of technology adoption issues in healthcare and gain a tool for evaluation of technology adoption potential of medical devices, which would potentially help improve clinician satisfaction with healthcare technology and aid in betterment of quality of care. The learnings may lead to better incentives and programs for clinicians and help overcome certain adoption factors in their pervasive technology implementations. The model is generalizable and can ultimately be applied to benefit many other types of organizations.

Your name and responses will be confidential and will not be shared with any third party. Any data linked to your identification will be stored in a secured place only accessible by the researcher. The data will be destroyed within one year after the completion of the study. Participation in this research is totally voluntary and you have the right to withdraw at any time without affecting your relationship with the researcher or any institute.

If you have concerns or problems about your participation in this study or your rights as a research subject, please contact PSU Institutional Review Board, Research Integrity, Research \& Strategic Partnerships, 1600 SW 4th Ave., Market Center Building, Ste. 620, Portland, OR 97201, (503) 7252227 or 1 (877) $480-4400$. If you have any questions about the study itself, contact Liliya Hogaboam:

Your signature indicates that you have read and understand the above information and agree to take part in this study. Please understand that you may withdraw your consent at any time without penalty, and that by signing, you are not waiving any legal claims, rights or remedies.

\section{Signature (Type} your name)

Date $(\mathrm{mm} / \mathrm{dd} / \mathrm{yyyy})$ 


\section{Assessment of Technology Adoption Potential of Medical Devices: Case of Wearable Sensor Products for Pervasive Care in Neurosurgery and Orthopedics}

Wearable medical devices that track the mobility of patients after spinal procedures could help surgeons in providing post-operative care, analysis of treatment outcomes and patient monitoring.

The objective of this research is to assess the potential for technology adoption of those wearable devices through a multi-perspective approach and development of a hierarchical decision-making model (HDM) that incorporates the relevant criteria and subcriteria encompassing the needs of a hospital neurological surgery and orthopedics department.

The development of the model components known as criteria, subcriteria and alternatives has been done through a comprehensive literature review.

The objective of this instrument is to qualify relative importance of decision variables through a numerical quantification process, which will require you to compare decision variables in a pairwise fashion. In case you might have difficulty with the terms, please refer to the explanations for the terms listed.

Please refer to figure below for more information about the model:

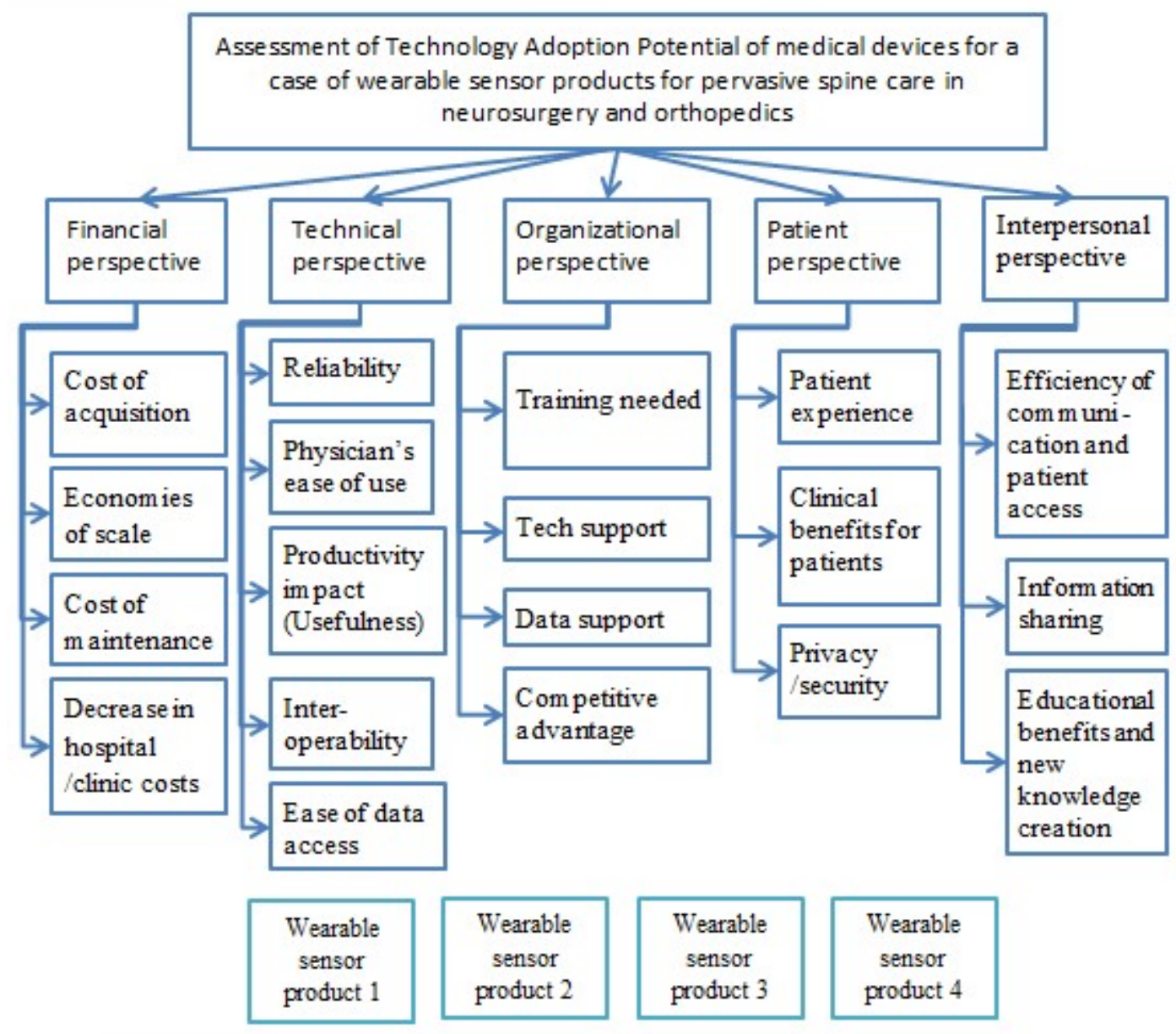


A literature review has revealed that there are five major perspectives associated with health information technology assessment. Those perspectives have relative importance values for the stakeholders. A brief explanation of each is presented below:

Financial perspective is mentioned in the literature from rising costs of healthcare to high costs of systems implementation and management as well as losses from unsuccessful software applications. It encompasses the importance of financial benefits to the organization that a certain technology selection could bring.

Technical perspective represents the value of a technology in a healthcare setting from the view of the importance of technical capabilities improvement.

Organizational perspective encompasses the criteria that technological solutions should provide in organizational setting, which includes training needed, tech support, data support as well as competitive advantage it can provide for the healthcare organization.

Patient perspective comprises of criteria that reflect patient issues of adoption of medical devices like satisfactory patient experience, clinical benefits and privacy/security of the information.

Interpersonal perspective reflects the importance of sharing and communication among various levels in the organization and outside, the ability of the medical wearable device to provide that capability and perhaps improve the communication and important flow of information.

In the following pairwise comparison scales, please rate the main criteria with respect to one another. The slider scale is given for this purpose.

For example, if you feel like 'Financial' is $\mathbf{4}$ times less important than 'Technical' in the following question, move the slider to 20. The number on the right side of the slider shows the importance of 'Financial perspective' compared to 'Technical perspective'. This would mean that given a scale of 100, Financial : Technical 20:80.

Equal importance would mean giving equal scores of 50:50. If you feel like 'Financial perspective' is more important than 'Technical perspective', give it a score of more than 50, for example 55:45 (1.22 times more important), 60:40 (1.5 times more important), 85:15 or 90:10 etc.. 
Important: For each pair a total of 100 is used. Scores of 0:100 and 100:0 are INVALID. The lowest score of 1:99 and 99:1 indicate negligible importance of one criteria in comparison with the other and vice versa.

\section{Evaluation of Main Perspectives}

Please distribute 100 points between the following pairs (pairwise comparison) of main wearable technology assessment perspectives to reflect your judgement on their relative importance to overall mission -- Assessment of Technology Adoption Potential of medical devices for a case of wearable sensor products for pervasive spine care in neurosurgery and orthopedics:

1. THE IMPORTANCE OF 'Financial perspective' WITH RESPECT TO 'Technical perspective' :

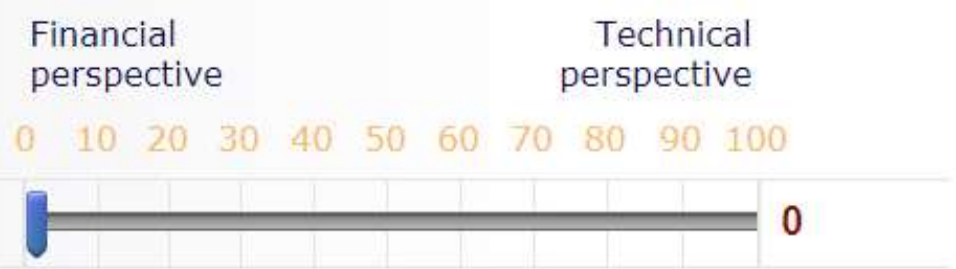

In the following pairwise comparison scales, please rate alternatives with respect to one another. The slider scale is given for this purpose.

For example, for the main goal of technology adoption, if you believe that 'VITALITI' is 1.5 times less desirable for adoption than 'VitalPatch' in the particular subcriterion category in the question, move the slider to 40. The number on the right side of the slider shows the importance of 'VITALITI' compared to 'VitalPatch'. This would mean that given a scale of 100, VITALITY: VitalPatch 40:60.

Equal importance would mean giving equal scores of 50:50. If you feel like 'VITALITY' is more desirable for adoption than 'VitalPatch', give it a score of more than 50 , for example $55: 45$ (1.22 times more important), $85: 15$ or $90: 10$ etc..

Important: For each pair a total of 100 is used. Scores of 0:100 and 100:0 are INVALID. The lowest score of 1:99 and 99:1 indicate negligible importance of one criteria in comparison with the other and vice versa. 
Please express your expert opinion of the ranking of the wearable products for the Assessment of Technology Adoption Potential of wearable medical devices for pervasive spine care in neurosurgery and orthopedics with respect to the subcriterion at the top of the scale bars.

(Please use the slider to determine the score of the first alternative (shown bold in blue) compared to the second alternative with respect to the subcriterion at the top of the scale bars).

Reliability - A characteristic of a wearable sensor technological solution providing accurate measurements and analytic reports; consistent and stable performance, free of technical issues and problems.

\section{Reliability}

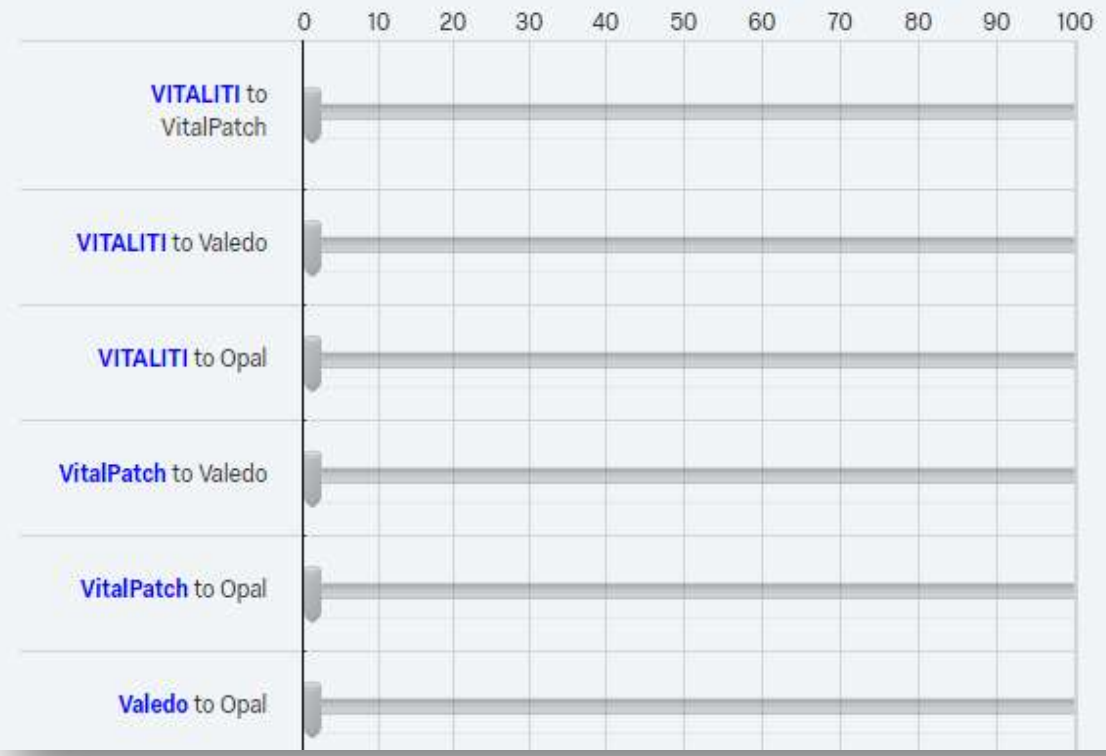




\section{Appendix D - XML Response File Sample}

$<$ ?xml version="1.0" encoding="UTF-8"?>

$-<\mathrm{xml}>$

$-<$ Response $>$

$<$ ResponselD>R_3k0ngyPdtpEs3cM </ResponselD>

$<$ ResponseSet $>$ Default Response Set $</$ ResponseSet $>$

$<$ Name $>$ Anonymous $</$ Name $>$

$<$ ExternalDataReference/>

$<$ EmailAddress/>

$<$ |PAddress/>

$<$ Status $>0</$ Status $>$

$<$ StartDate $>2017-05-23$ 07:32:00</StartDate $>$

$<$ EndDate $>2017-05-23$ 07:36:04</EndDate>

$<$ Finished $>1</$ Finished $>$

$<$ Q51 $>1</$ Q51 $>$

$<$ Q52_1_TEXT $\rangle^{* * * * * * * * * *}</$ Q52_1_TEXT $>$

$-<Q 522$ TEXT $>$

$<$ ! $\left[\mathrm{CDA} T \overline{\mathrm{A}}\left[{ }^{* *} / * * / * * * *\right]\right]>$

$</$ Q52_2_TEXT>

$<Q 6>1</ Q 6>$

$<$ Q29 $>1</$ Q29 $>$

$<$ Q30 $>1</ Q 30>$

$<Q 5>1</ Q 5>$

$<$ Q10 $1>30<$ Q10 1>

$<$ 29_1 $>29</ Q 29 \_1>$

$<$ Q30_1 $>81</ Q 30 \_1>$

$<Q 42$ 1 $>50</ Q 42 \_1>$

$<$ Q43_1 $>91</ Q 43 \_1>$

$<$ Q44_1 $>91</ Q 44 \_1>$

$<$ Q45_1 $>50</ Q 45 \_1>$

$<$ Q46 $1>91</ Q_{46} \quad 1>$

$<$ Q47 $1>91</ Q 47 \quad 1>$

$<$ Q48_1 $>20</ Q 48 \_1>$

$<$ Q49_1 $>$ 50 $</ Q 49$ 1 $>$

$<$ Q50_1 $>81</ Q 50 \_1>$

$<$ LocationLatitude/>

$<$ LocationLongitude/>

$<$ LocationAccuracy>-1</LocationAccuracy>

$</ x m \mid>$

$</$ Response> 


\section{Appendix E - Criteria Mapping xml file for Ruby and R}

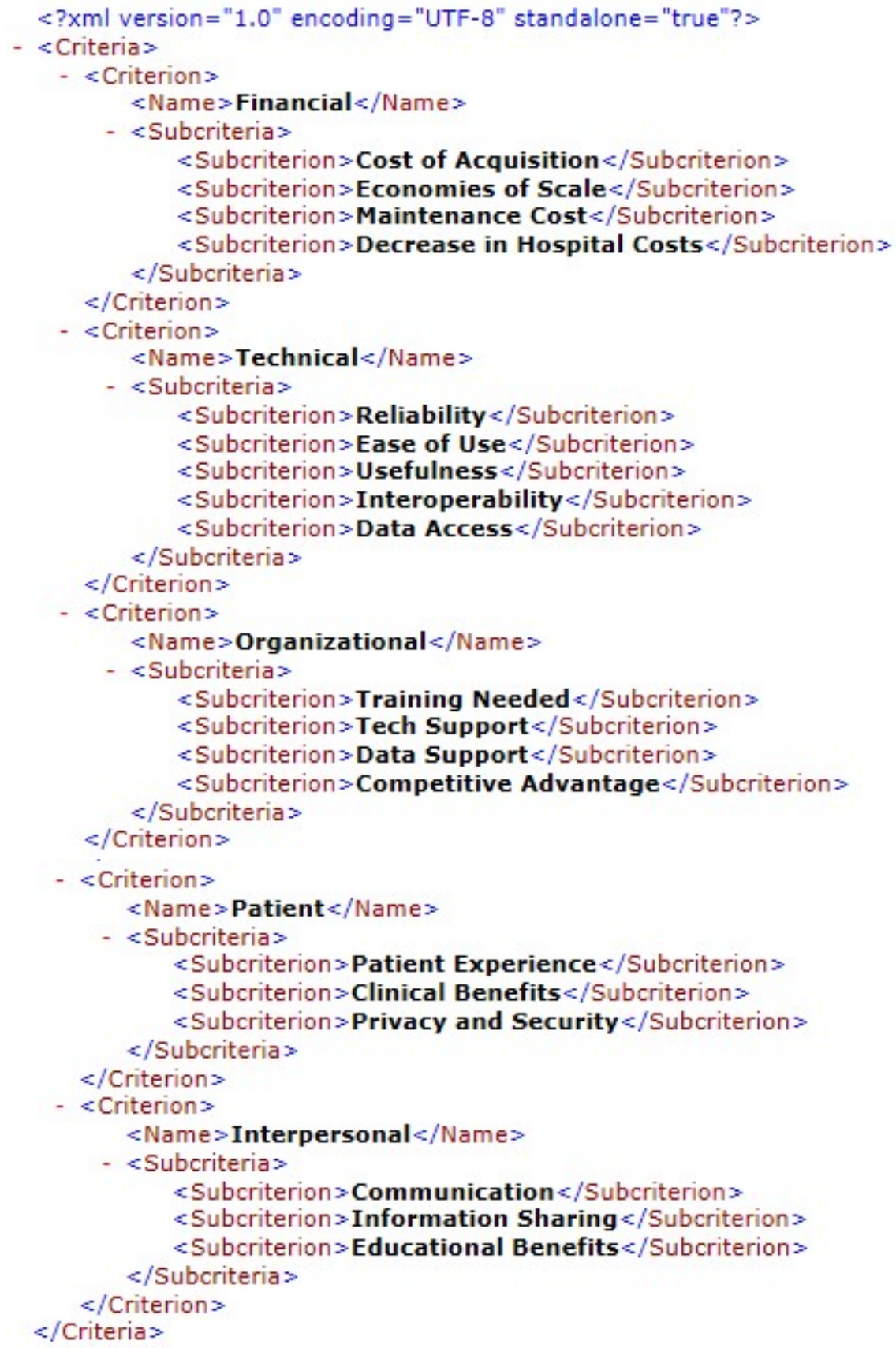




\section{Appendix F - Sample of Questions Mapping xml file}

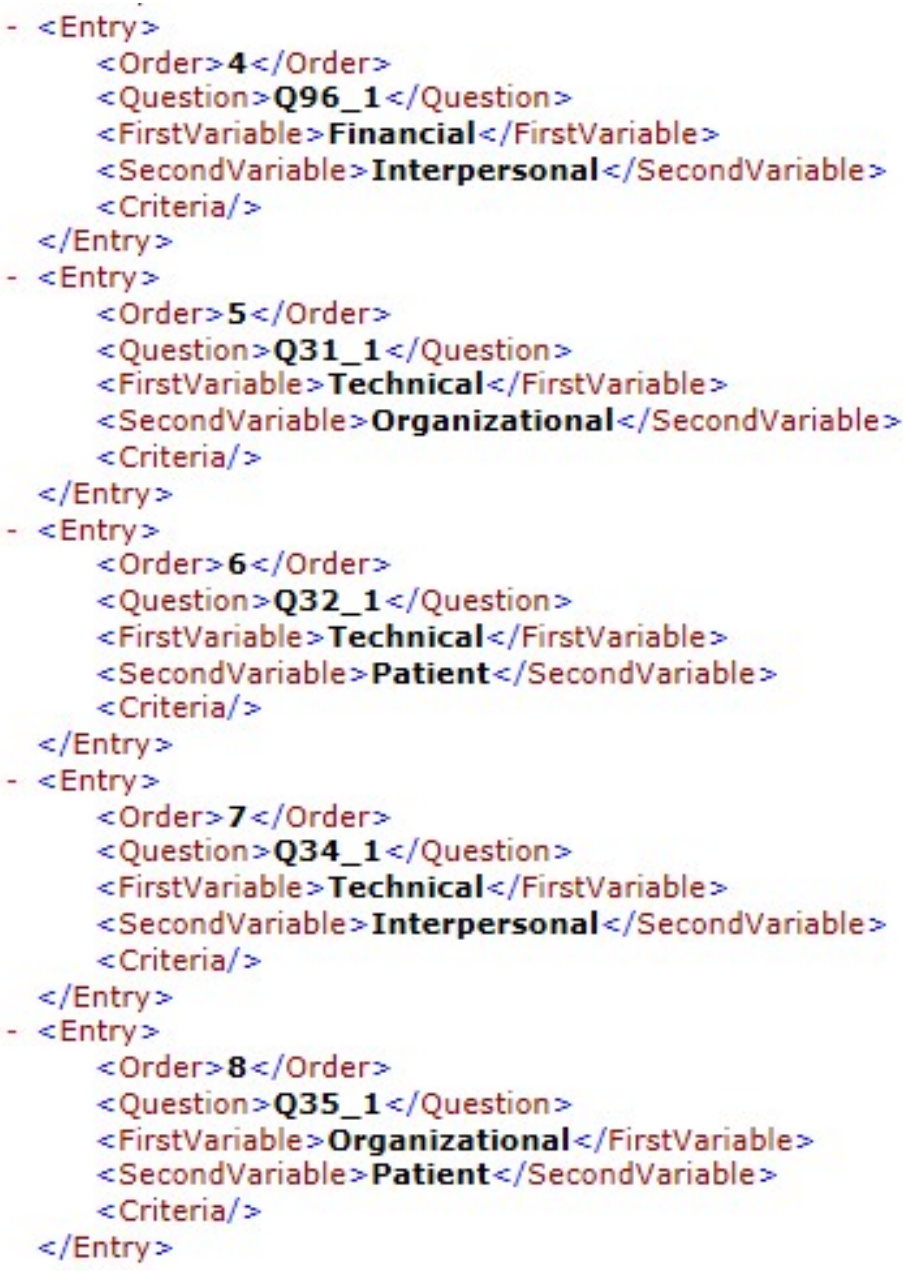




\section{Appendix G - Sample Hierarchical Decision Model code in R}

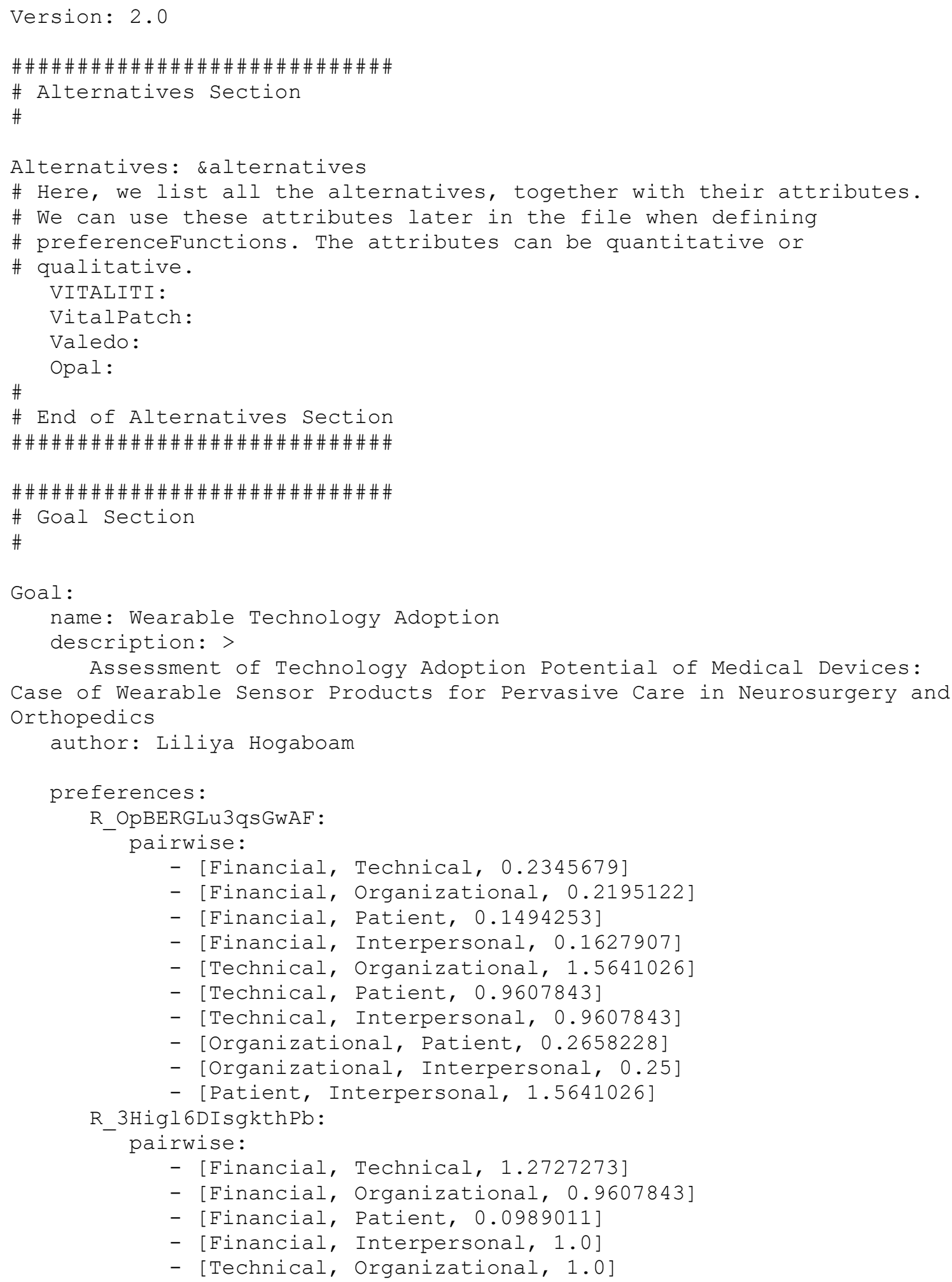


- [Technical, Patient, 0.3513514]

- [Technical, Interpersonal, 1.0]

- [Organizational, Patient, 0.4492754]

- [Organizational, Interpersonal, 0.9607843]

- [Patient, Interpersonal, 3.1666667]

R_1k16xlztDZMGaxv:

pairwise:

- [Financial, Technical, 4.5555556]

- [Financial, Organizational, 1.0]

- [Financial, Patient, 9.0]

- [Financial, Interpersonal, 4.0]

- [Technical, Organizational, 1.0]

- [Technical, Patient, 2.4482759]

- [Technical, Interpersonal, 0.6666667]

- [Organizational, Patient, 1.0]

- [Organizational, Interpersonal, 0.9607843]

- [Patient, Interpersonal, 0.25]

children:

Financial:

preferences:

R_OpBERGLu3qsGwAF :

pairwise:

- [VITALITI, VitalPatch, 1]

- [VITALITI, Valedo, 1]

- [VITALITI, Opal, 1]

- [VitalPatch, Valedo, 1]

- [VitalPatch, Opal, 1]

- [Valedo, Opal, 1]

R_3Higl6DIsgkthPb:

pairwise:

- [VItALITI, VitalPatch, 1]

- [VItALITI, Valedo, 1]

- [VItALITI, Opal, 1]

- [VitalPatch, Valedo, 1]

- [VitalPatch, Opal, 1]

- [Valedo, Opal, 1]

R_1k16xlztDZMGaxv:

pairwise:

- [VITALITI, VitalPatch, 1]

- [VItALITI, Valedo, 1]

- [VITALITI, Opal, 1]

- [VitalPatch, Valedo, 1]

- [VitalPatch, Opal, 1]

- [Valedo, Opal, 1]

children: *alternatives

Technical:

preferences:

R_OpBERGLu3qsGwAF :

pairwise:

- [VITALITI, VitalPatch, 1]

- [VITALITI, Valedo, 1]

- [VITALITI, Opal, 1]

- [VitalPatch, Valedo, 1]

- [VitalPatch, Opal, 1] 


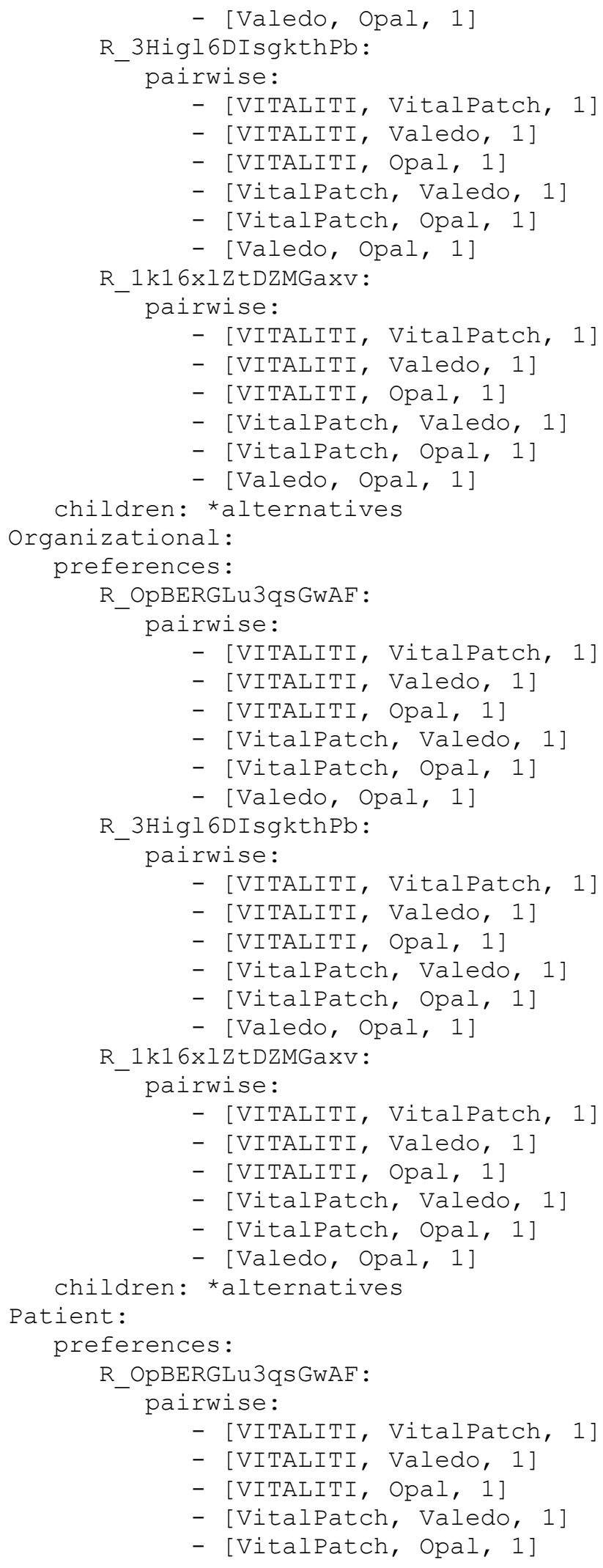


[Valedo, Opal, 1]

R_3Higl6DIsgkthPb:

pairwise:

- [VITALITI, VitalPatch, 1]

- [VItALITI, Valedo, 1]

- [VItALITI, Opal, 1]

- [VitalPatch, Valedo, 1]

- [VitalPatch, Opal, 1]

- [Valedo, Opal, 1]

R_1k16xlztDZMGaxv:

pairwise:

- [VITALITI, VitalPatch, 1]

- [VITALITI, Valedo, 1]

- [VItALIti, Opal, 1]

- [VitalPatch, Valedo, 1]

- [VitalPatch, Opal, 1]

- [Valedo, Opal, 1]

children: *alternatives

Interpersonal:

preferences:

R_OpBERGLu3qsGwAF :

pairwise:

- [VITALITI, VitalPatch, 1]

- [VITALITI, Valedo, 1]

- [VITALITI, Opal, 1]

- [VitalPatch, Valedo, 1]

- [VitalPatch, Opal, 1]

- [Valedo, Opal, 1]

R_3Higl6DIsgkthPb:

pairwise:

- [VITALITI, VitalPatch, 1]

- [VItALITI, Valedo, 1]

- [VITALITI, Opal, 1]

- [VitalPatch, Valedo, 1]

- [VitalPatch, Opal, 1]

- [Valedo, Opal, 1]

R_1k16xlztDZMGaxv:

pairwise:

- [VITALITI, VitalPatch, 1]

- [VITALITI, Valedo, 1]

- [VITALITI, Opal, 1]

- [VitalPatch, Valedo, 1]

- [VitalPatch, Opal, 1]

- [Valedo, Opal, 1]

children: *alternatives

\#

\# End of Goal Section

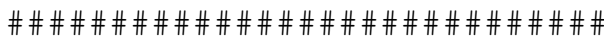




\section{Appendix H - HDM Research Software module}

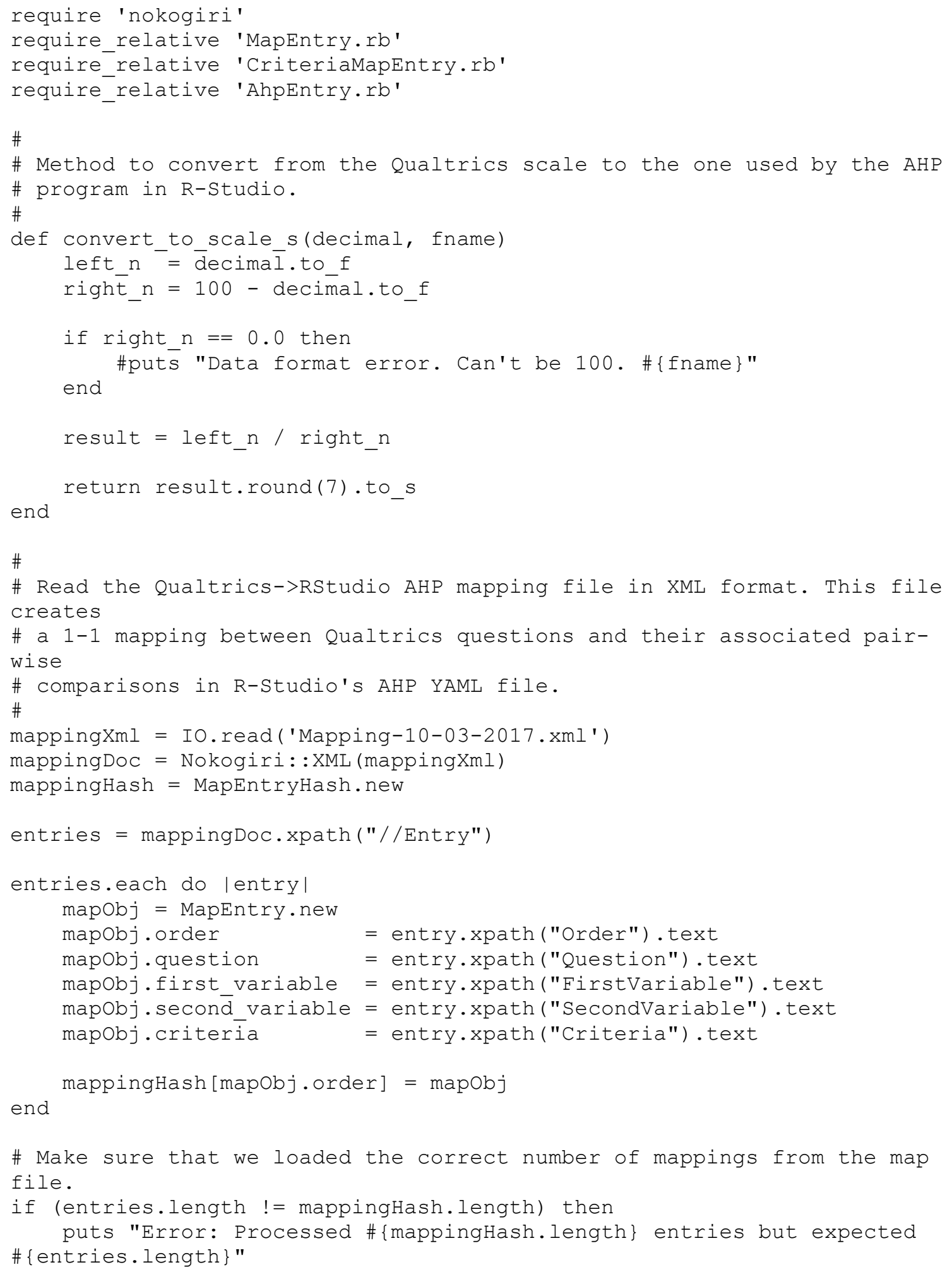




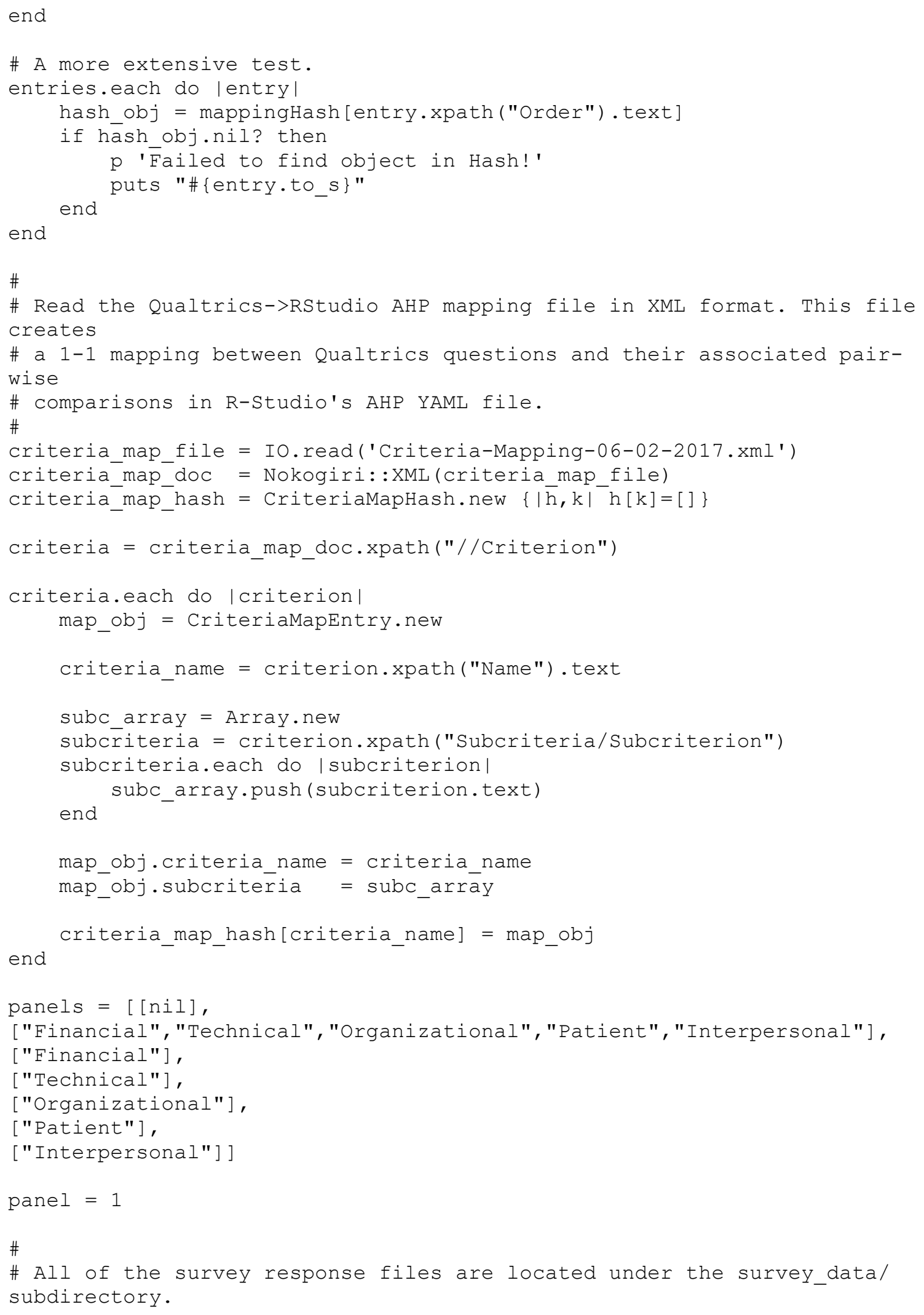




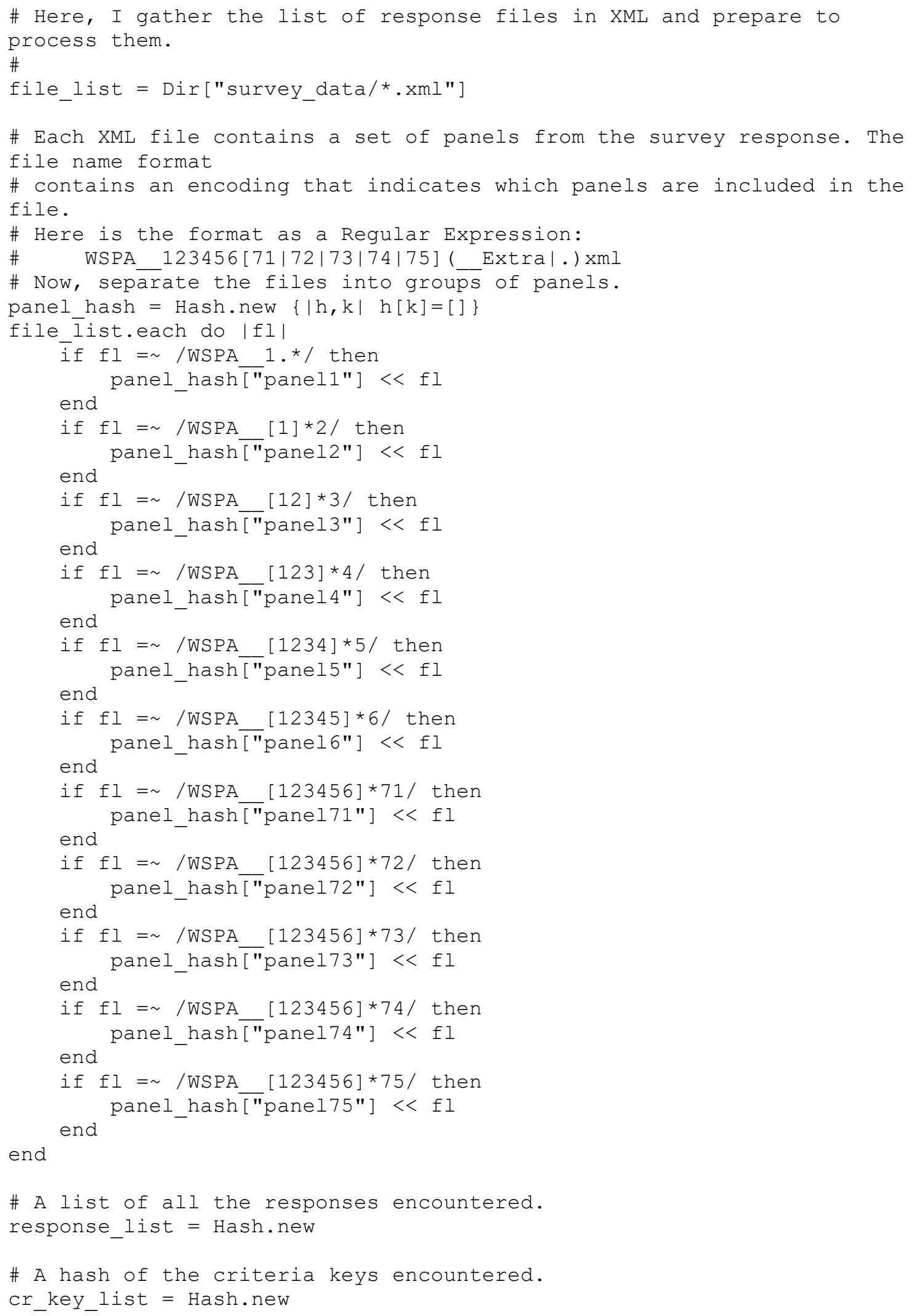




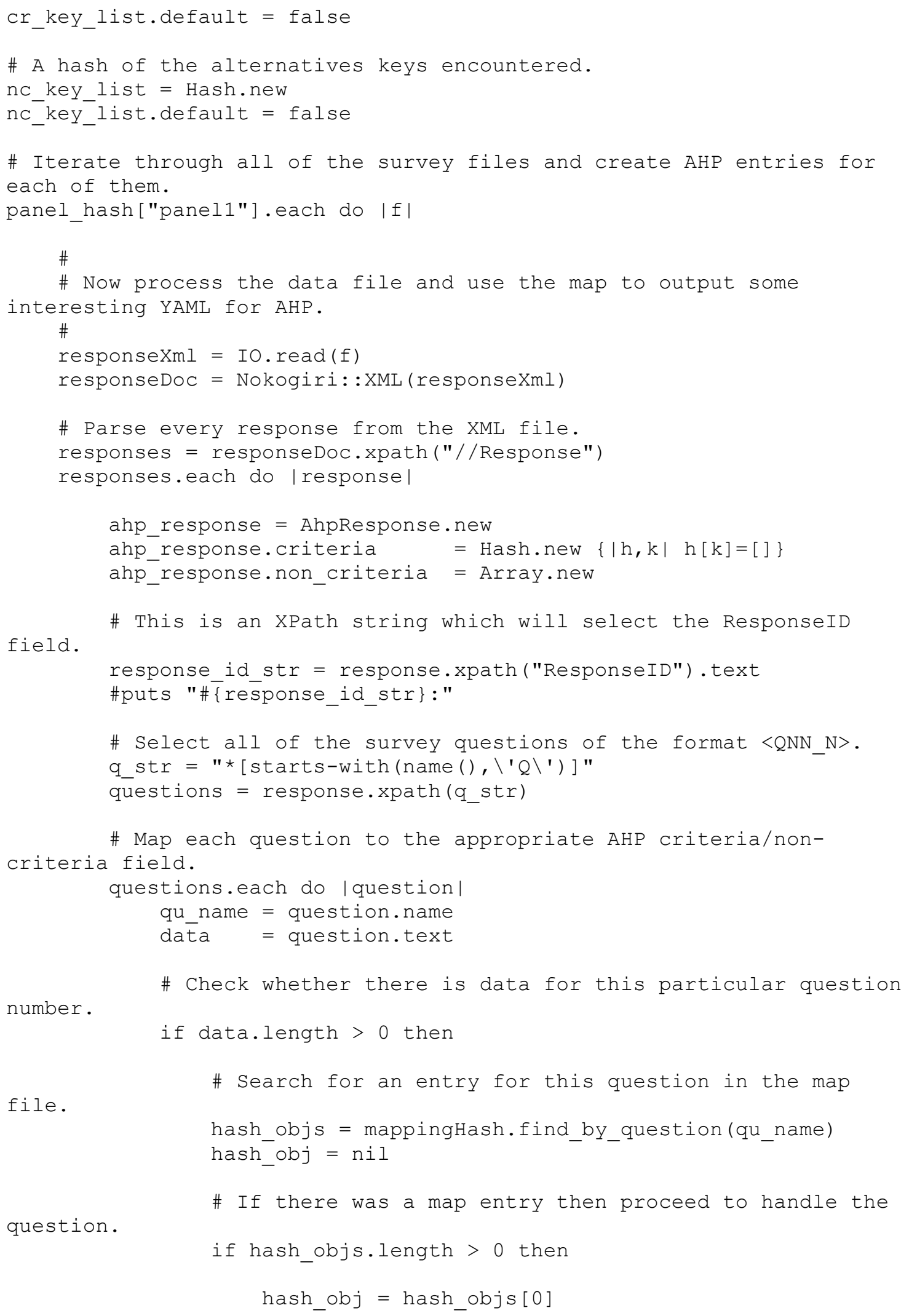




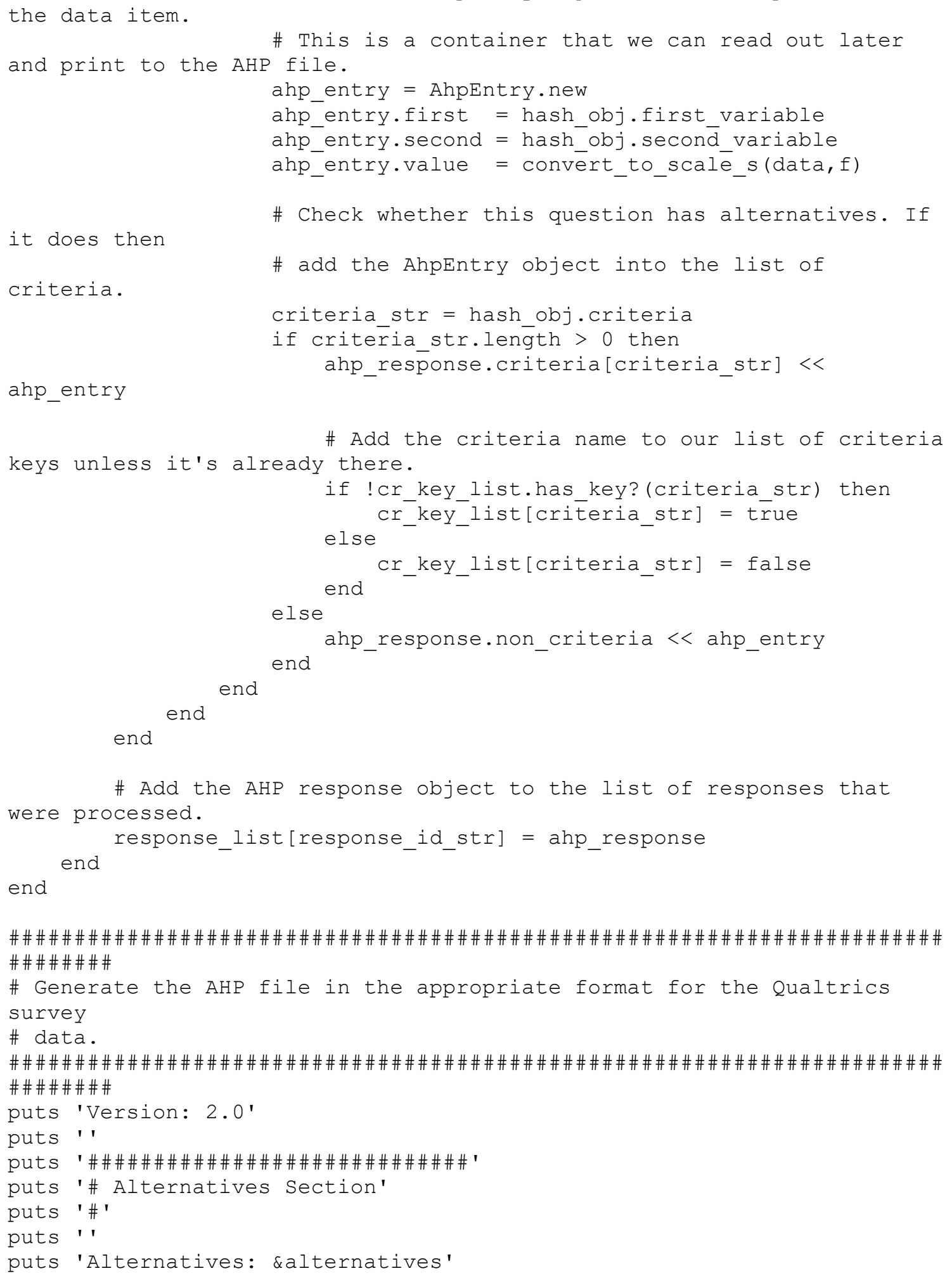




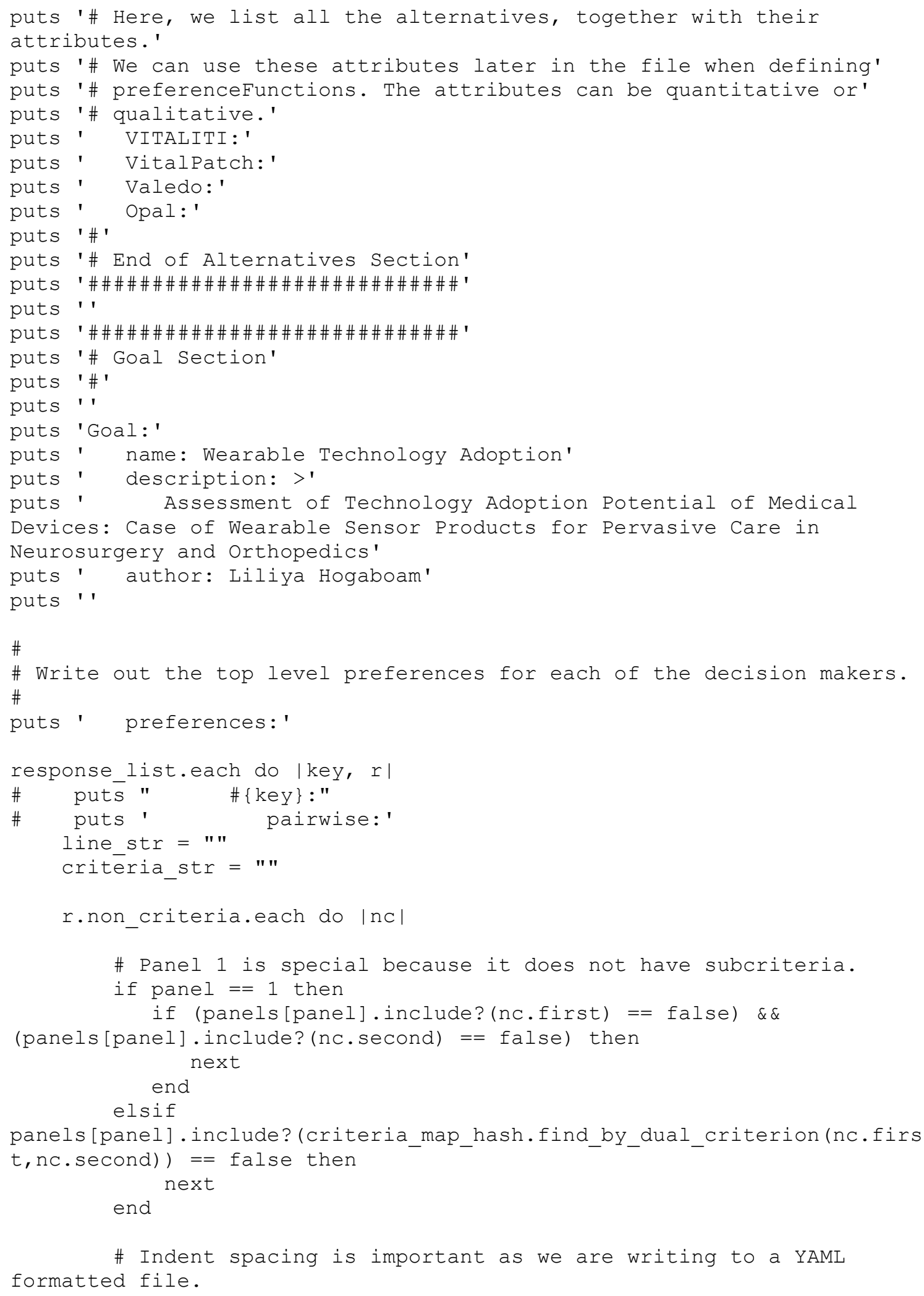




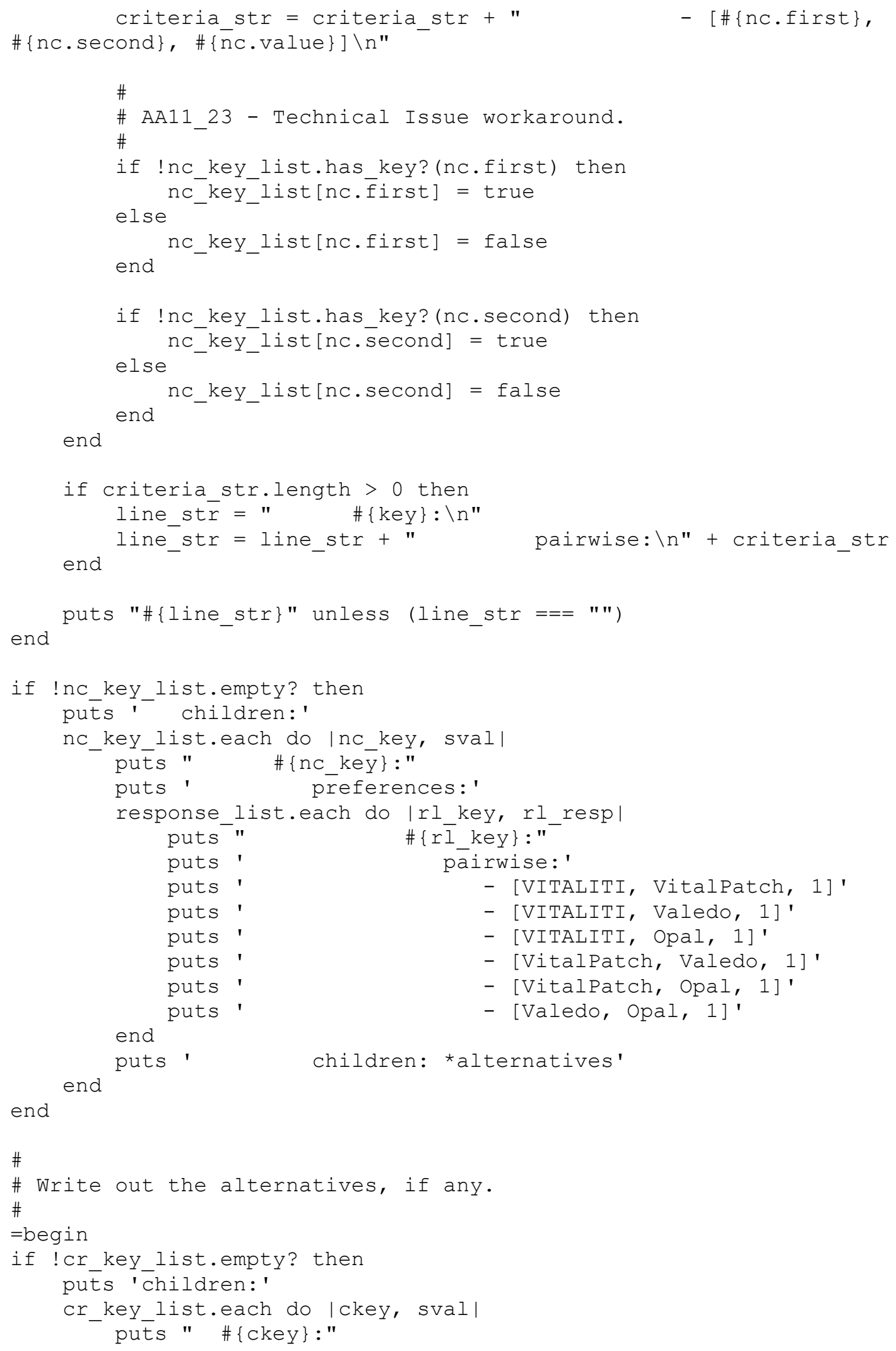




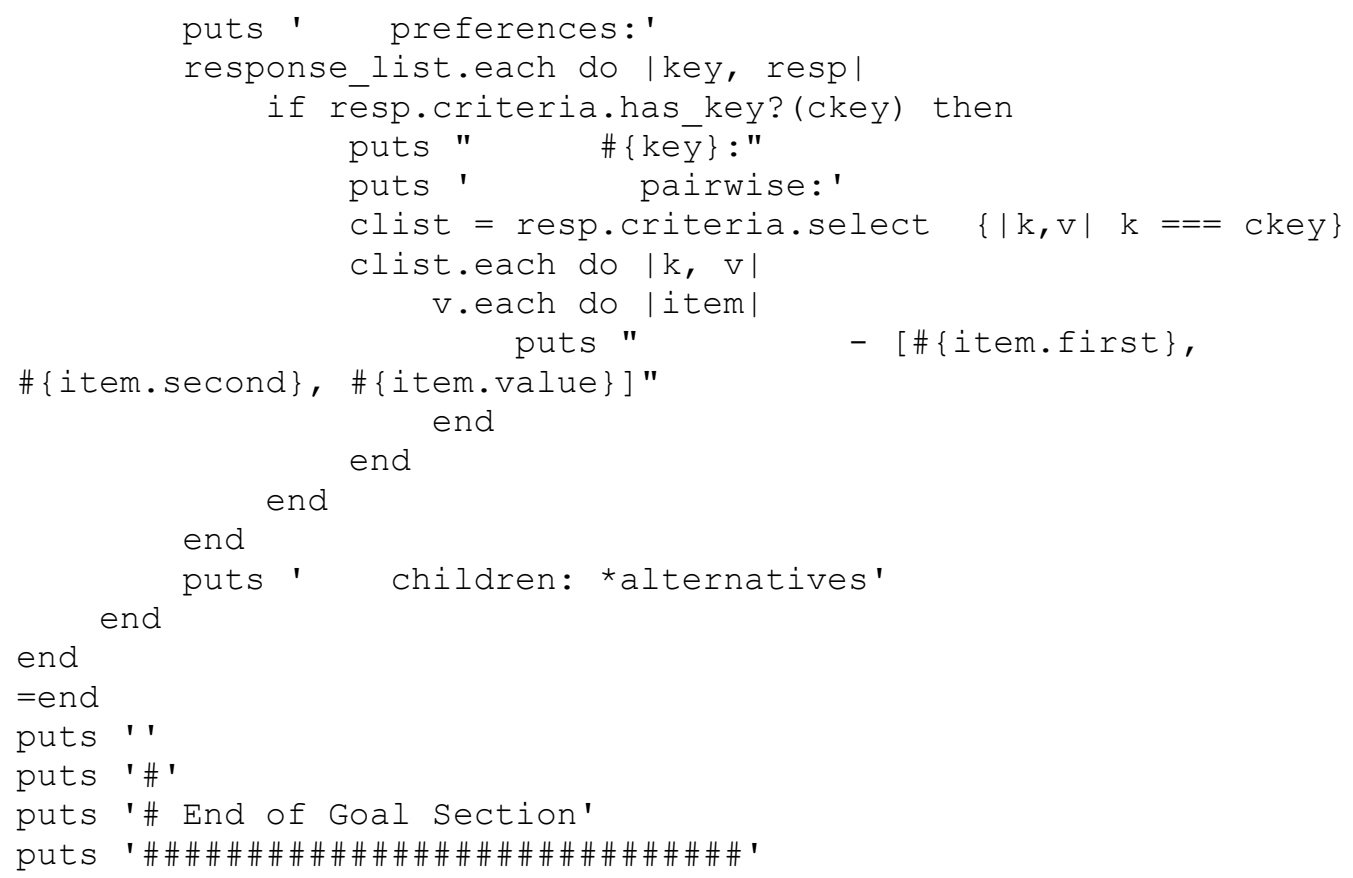

